\title{
HYBRID SILICON-BASED NANOMATERIALS SYNTHESIZED \\ VIA FEMTOSECOND LASER FOR THERANOSTICS \\ APPLICATIONS
}

\author{
by \\ Meysam Keshavarz \\ Master of Science, University Technology of Malaysia (2012) \\ Bachelor of Science, Esfahan Azad University (2010)
}

\author{
A dissertation \\ presented to Ryerson University \\ in partial fulfillment of the \\ requirements for the degree of \\ Doctor of Philosophy \\ in the program of \\ Aerospace Engineering
}

Toronto, Ontario, Canada, 2017

(C) Meysam Keshavarz, 2017 


\section{AUTHOR'S DECLARATION FOR ELECTRONIC SUBMISSION OF A DISSERTATION}

I hereby declare that I am the sole author of this dissertation. This is a true copy of the dissertation, including any required final revision, as accepted by my examiners.

I authorize Ryerson University to lend this dissertation to other institutions or individuals for the purpose of scholarly research.

I further authorize Ryerson University to reproduce this dissertation by photocopying or by other means, in total or in part, at the request of other institutions or individuals for the purpose of scholarly research.

I understand that my dissertation may be made electronically available to the public. 


\title{
HYBRID SILICON-BASED NANOMATERIALS SYNTHESIZED VIA FEMTOSECOND LASER FOR THERANOSTICS APPLICATIONS
}

\author{
Meysam Keshavarz \\ Doctor of Philosophy, Aerospace Engineering, Ryerson University, Toronto (2017)
}

\section{ABSTRACT}

Biocompatibility and bio-stability are two very important criteria of the materials being used in biology and biological related applications. Among biocompatible materials, Silicon has been widely used in biomedical applications such as logistic for drug delivery and scaffolds for tissue engineering. Therefore, Silicon is chosen to be further investigated in terms of its potential therapeutic and diagnostic applications. The main objective of this thesis is to explore capabilities of silicon-based nanomaterials on therapeutic and diagnostic of cancer HeLa and mammalian Fibroblast cell lines. The uniqueness of silicon-based nanomaterials synthesized in this study relies on utilization of femtosecond laser, a versatile yet precise method to generate nanoscale siliconbased materials.

In the initial phase of this study, the need for manufacturing an alternative tool to modulate cells behavior is addressed. During this phase, it is shown that Induced Residual Stress (IRS) onto the Silicon chip via Ultrashort Pulsed Laser (USPL) irradiation is remarkably capable of remotely tuning cellular behavior by which the fate and directionality of seeded cells could be modulated. The second phase is dedicated to synthesis of a novel nanostructure to overcome the limitations of using Silicon-based bio-template for therapeutic applications. Thus far, Nano-scale silicon material has been employed in the form of either nanoparticles (NPs) or 3-D structure for drug delivery and scaffold respectively. Here, a self-assembled Silica Nano-web (SNW) that concurrently exhibits the therapeutic and proliferative attributions of NPs and 3-D structure is 
introduced. This SNW also demonstrates selective functionality by which mammalian cells and cancer cells are treated differently.

The third phase of this study focuses on diagnostic applications of the silicon-based materials. In this phase, a label-free multiplex photoluminescent silicon nano-probe (PLSN-probe) as a potential substitute for quantum dots (QDs) in bioimaging is synthesized. An inherently nonphotoluminescent silicon substrate is altered to create the PLSN-probe, which overcomes the major drawbacks of presently available QDs. The PLSN-probe not only demonstrates unique optical properties that can be utilized for bioimaging but also exhibits cell-selective uptake that allows the differentiation and diagnosis of HeLa and fibroblast cells.

Last but not least, the final study is a unique approach of using polygonal Silicon quantum-probe (polygonal Si-QP) that utilizes a surface-enhanced Raman scattering (SERS) for diagnosis and differentiating of cancerous HeLa and fibroblast cells. It is demonstrated that the polygonal SiQPs could be used for in-situ live cell analysis. Live Raman sensing has also exhibited promising outcome by which tracing of chemical transition in a cell could lead us to a better understanding of cellular changes.

The obtained results indicate multifunctional feasibility of nano/quantum scale silicon based structures for therapeutic, bioimaging and diagnosis. The prospect applications of this thesis could lead to future studies and ultimately, new frontiers for universal cancer cell diagnosis. 


\section{ACKNOWLEDGEMENTS}

First and foremost, would like to express my gratitude and appreciation for my supervisors Dr. Krishnan Venkatakrishnan and Dr. Bo Tan. It has been an honor to conduct my study under their supervision. I appreciate all the contributions of time, ideas, and funding to make my Ph.D. experience productive and stimulating.

I would like to thank my committee members Dr. Z. Fawaz, Dr. P. Ouyang and Dr. S. Upreti for their valuable guidance and meticulous advices to shape the direction of my research project and refining my thesis.

I would like to thank my parents. Words are unable to express the depth of my gratitude for their constant encouragement and support throughout the course of my entire academic career. I would also like to thank my sister Toktam for helping me whenever I needed any helps. Without them I could not have done this. 


\section{DEDICATION}

To my parents and my sister. 


\section{TABLE OF CONTENTS}

ABSTRACT ............................................................................................................................... iii

DEDICATION ........................................................................................................................ vi

LIST OF FIGURES ................................................................................................................

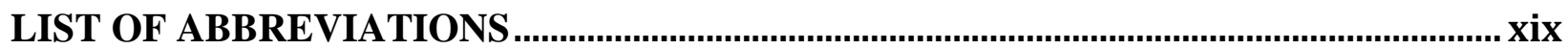

CHAPTER 1 ..............................................................................................................................1

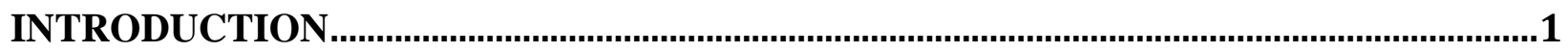

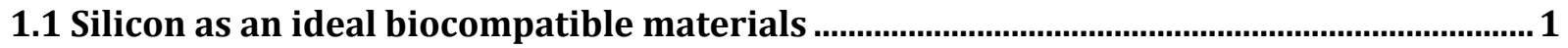

1.2 Silicon based nanomaterials for biological applications …............................................ 1

1.2.1 Nanostructured materials for tissue engineering ........................................................................ 2

1.2.2 Conventional trends on synthesis of Silicon nano materilas ........................................................... 2

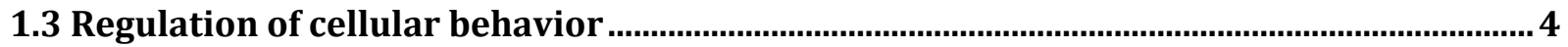

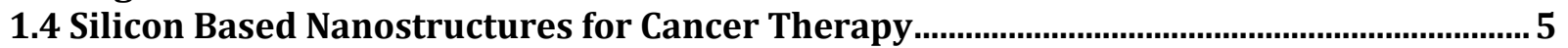

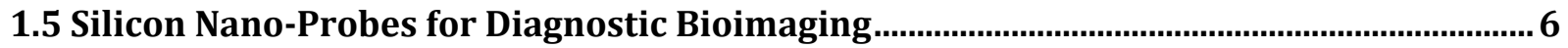

1.6 Silicon Quantum Probe towards Cancer Diagnosis ............................................................... 8

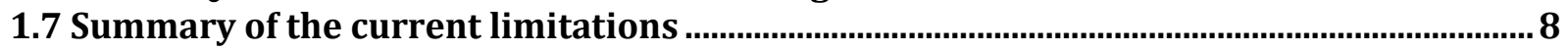

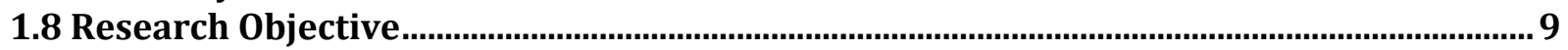

1.8.1 To determine the interaction of cell-matter and modulation of cellular behavior .......................... 9

1.8.2 To synthesize polymorphic 3-D silica nanostructures....................................................................... 9

1.8.3 To generate a multiplex PhotoLuminescent probe for cell-imaging and diagnosis ....................... 9

1.8.4 Fabrication of Raman sensitive probe for cancer diagnosis .............................................................. 9

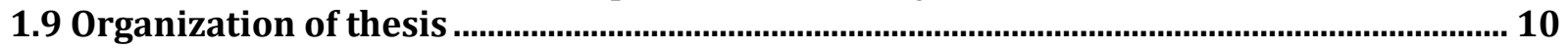

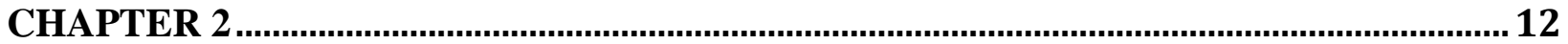

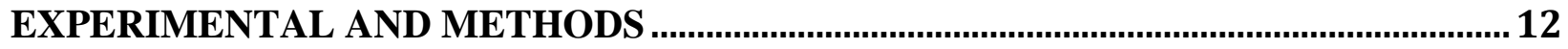

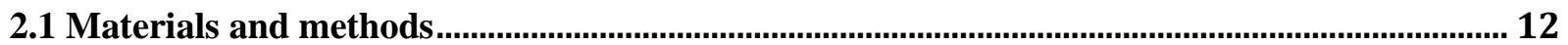

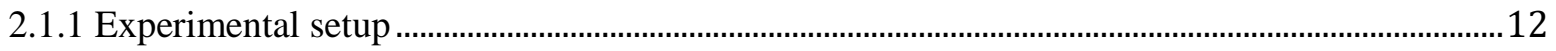

2.1.2 Laser induced functionalized stress component ...........................................................................12

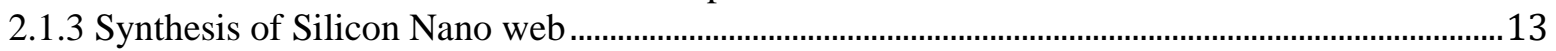

2.1.4 Synthesis of photoluminescence silicon nano probes...................................................................13

2.1.5 Synthesis of the SERS-enabled polygonal Si-QPs ..........................................................................13

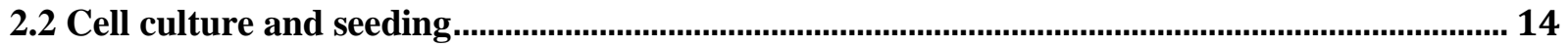

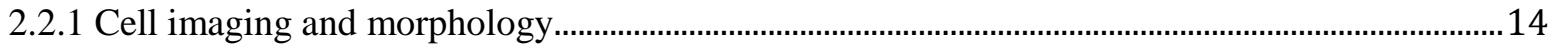

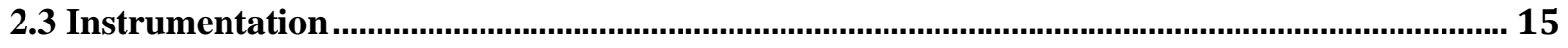

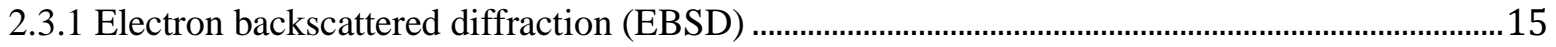

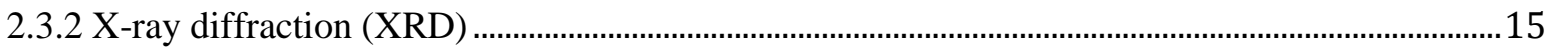

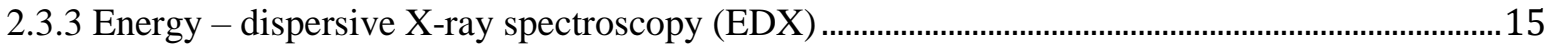

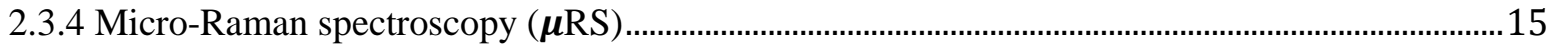

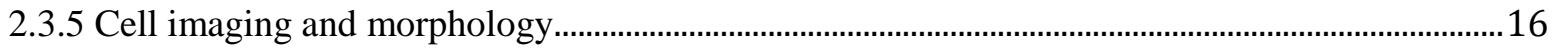

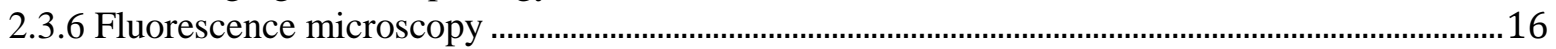

2.3.7 Morphology and physicochemical characterization.........................................................................16

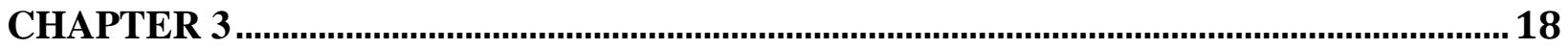

FUNCTIONALIZED STRESS COMPONENT ONTO BIO-TEMPLATE AS A

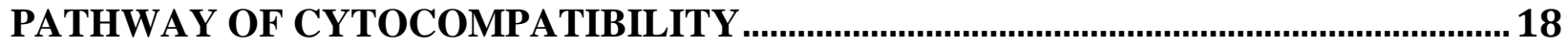




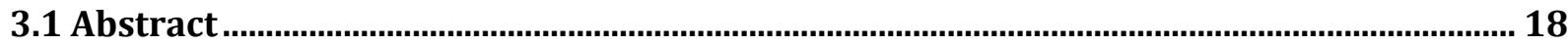

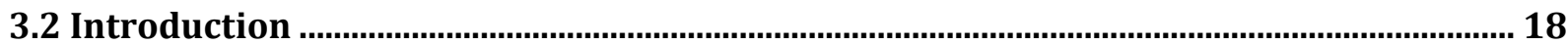

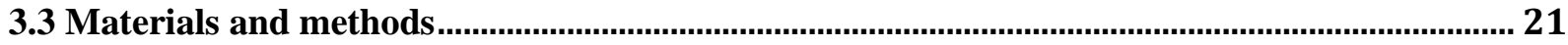

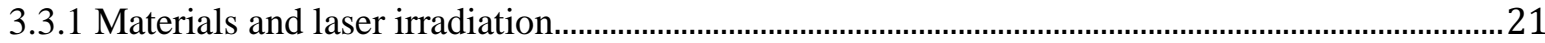

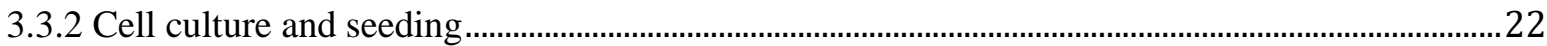

3.3.3 Cell imaging and morphology

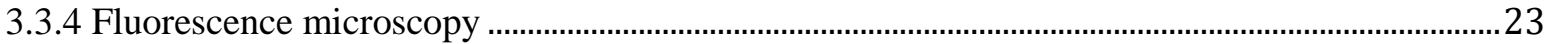

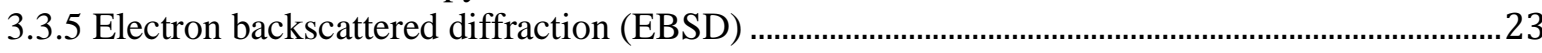

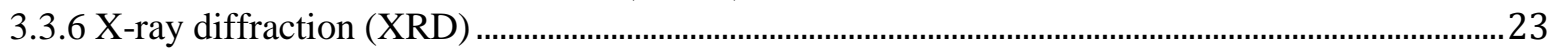

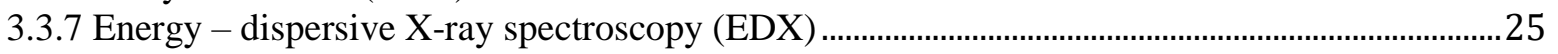

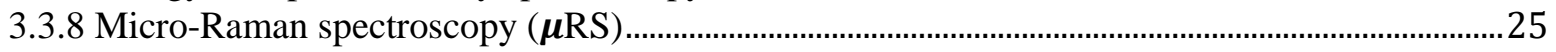

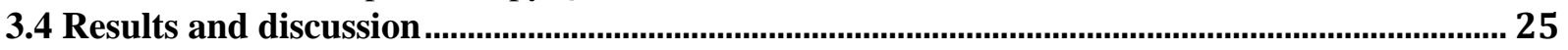

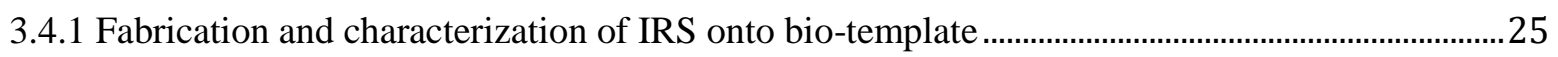

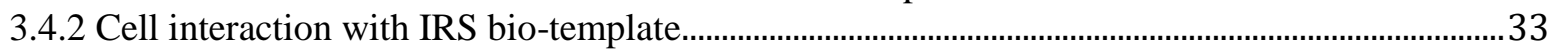

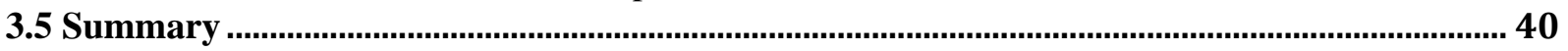

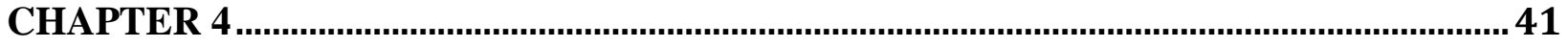

POLYMORPHIC SELF-ASSEMBLED SILICA NANO-WEBS AS A HELA-CENTRIC BIO-TEMPLATE TOWARD AN INTENSIFIED APOPTOSIS PATHWAY .................... 41

4.1 Abstract

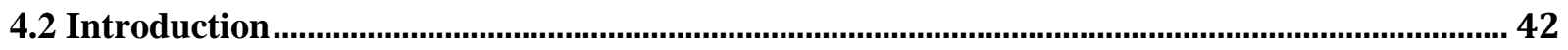

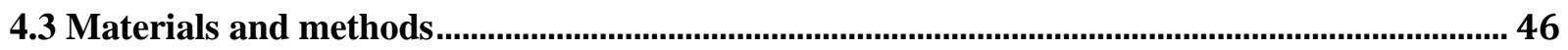

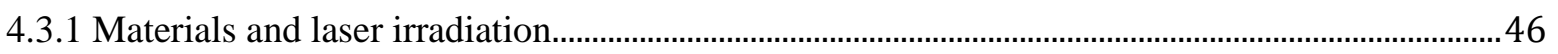

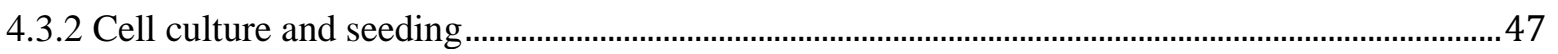

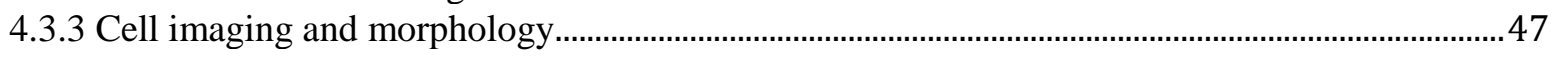

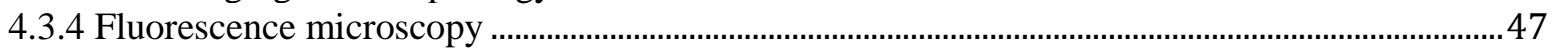

4.3.5 Morphology and physicochemical characterization........................................................................... 48

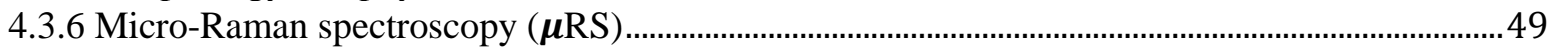

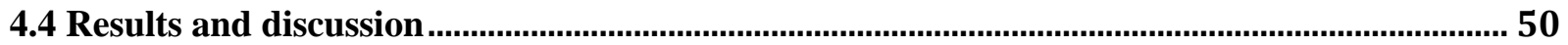

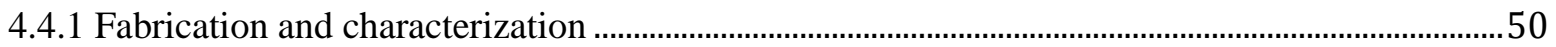

4.4.2 Cellular Response to Silica Nano-webs ............................................................................................ 54

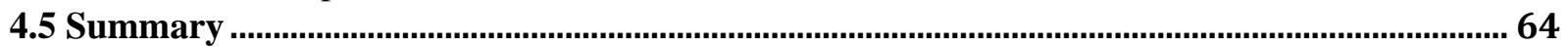

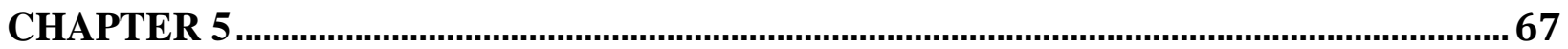

MULTIPLEX PHOTOLUMINESCENT SILICON NANO-PROBE FOR DIAGNOSTIC

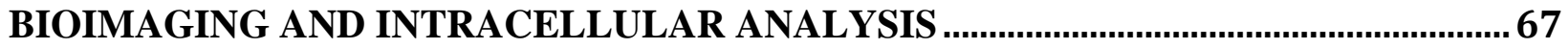

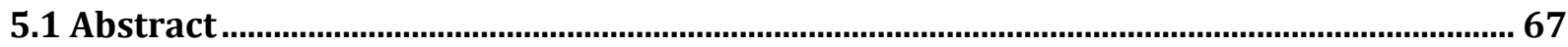

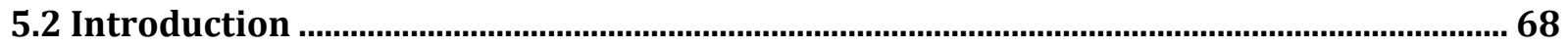

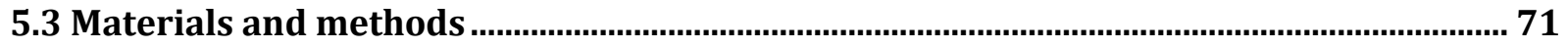

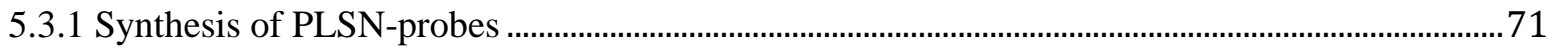

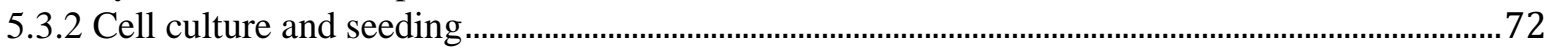

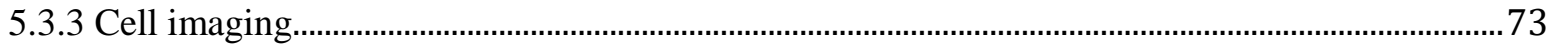

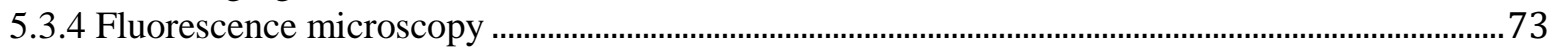

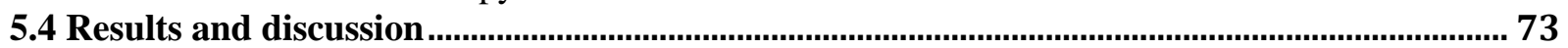

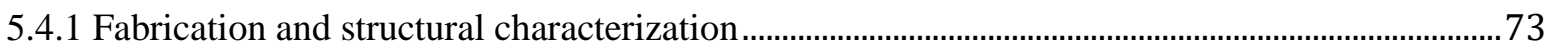

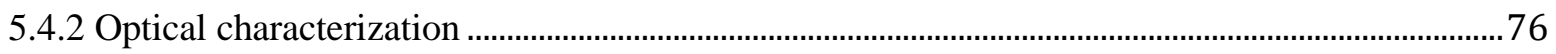

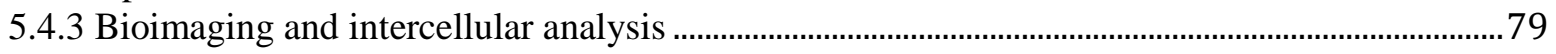

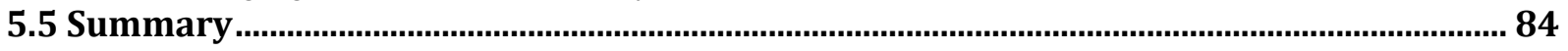


SURFACE ENHANCED RAMAN SPECTROSCOPY (SERS)-ENABLED POLYGONAL SILICON QUANTUM PROBE TOWARDS CANCER DIAGNOSIS .................................. 86

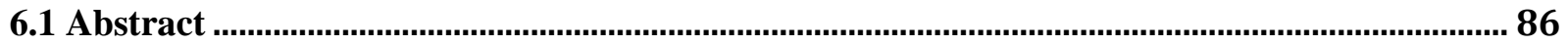

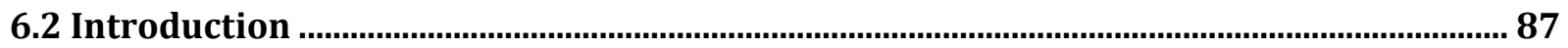

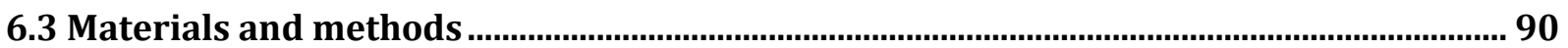

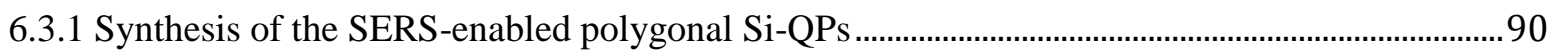

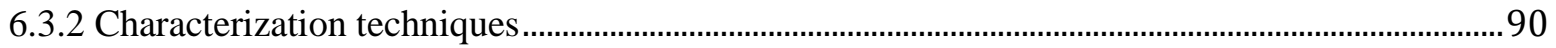

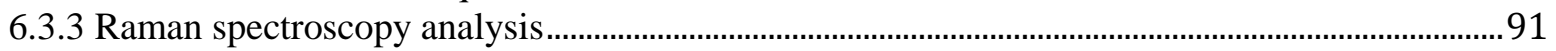

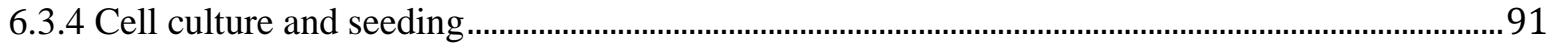

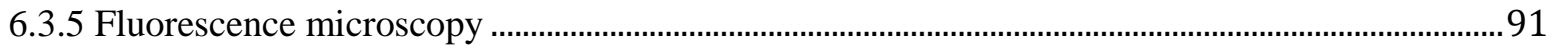

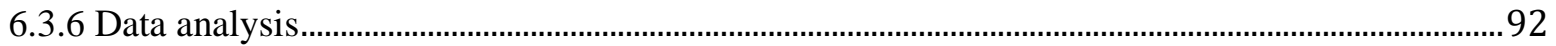

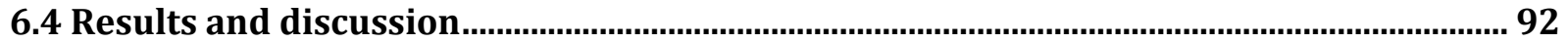

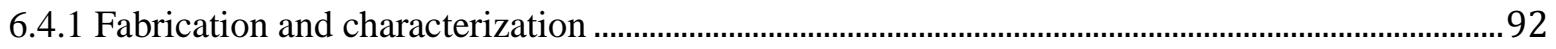

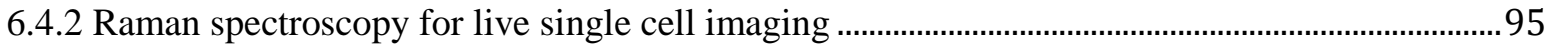

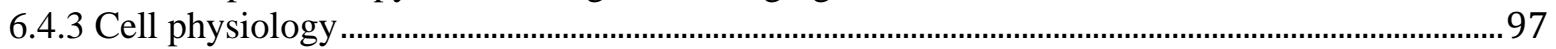

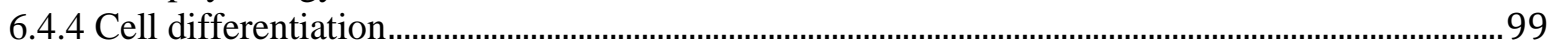

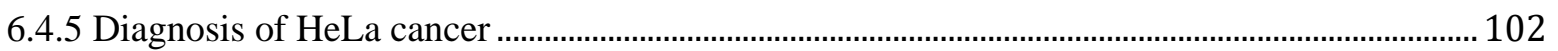

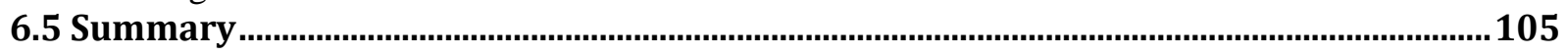

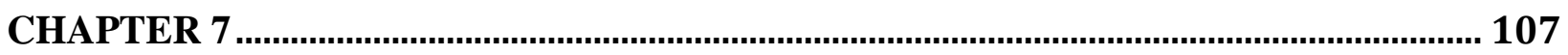

CONCLUSION .............................................................................................................................. 107

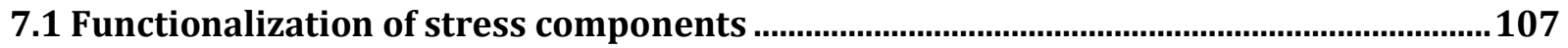

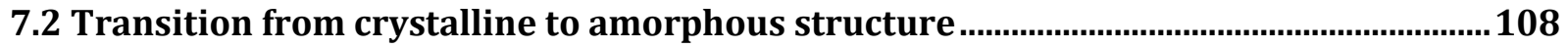

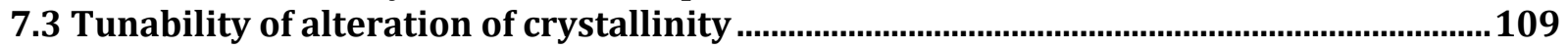

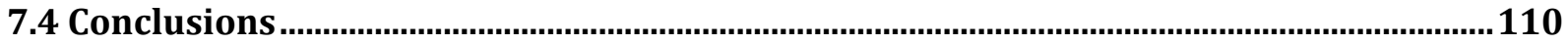

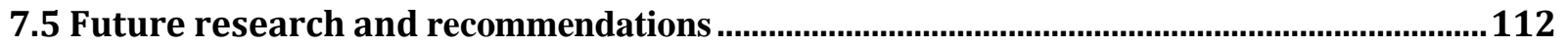

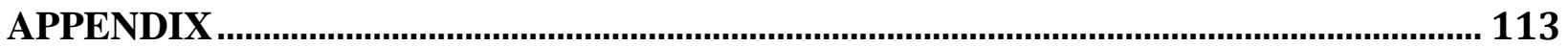

Appendix 1 Influence of suspended amorphous nanostructures......................................................... 113

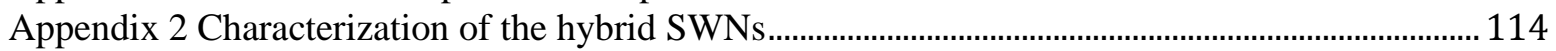

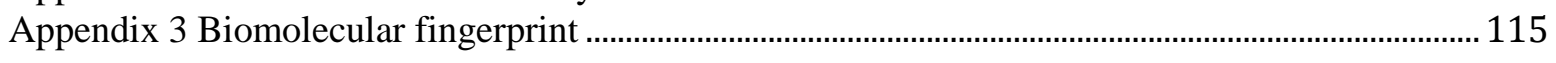

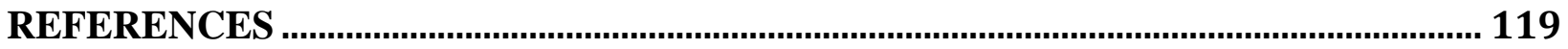




\section{LIST OF FIGURES}

Figure 1.1: Schematic of cell pattering by means of micro-stamp (1) and micro-grooves (2) (Courtesy)

Figure 1.2: Demonstration of using nanoparticles as drug carrier (1). Interaction of tagged nanocarrier with the targeted cell (2). (Courtesy).....

Figure 1.3: Scheme (1) illustrates the relation between the energy level and processes of exciton creation. (2) Exhibits the size dependency of quantum dots on the emitted light (Courtesy) ....... 6

Figure 1.4: Scheme illustration of SERS-enabled Si probe by localized surface plasmon .......... 7

Figure 3.1: Schematic illustration shows the response of cells to IRS. Two types of encounters on the proximity of IRS are: cell breakage and cell protrusion. SEM images of HeLa and Fibroblast (NIH3T3) represent collective cell migration towards stress-free area on the bio-template.

Figure 3.2: Plot A, shows a variation of laser fluence versus ablation threshold of Silicon biotemplate a distinct laser pulse width of (214 fs) and repetition rates ranging from 4 to $26 \mathrm{MHz}$. Plot B, Temperature variation with rep.rate, shows temperature rise exponentially by increasing laser rep.rate. Plot $\mathrm{C}$, shows the temperature vs. heat dissipation time. Figures $\mathrm{D}$ to $\mathrm{G}$ show the temperature gradient simulated by COMSOL Multiphysics. (All the data were taken after 10

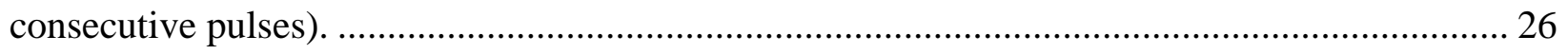

Figure 3.3: A, Backscatter scanning area. B, EBSD mapping of the IRS zone. C, EBSD relative Euler orientation maps. (The color is related to the crystallographic direction that is parallel to the surface). 29

Figure 3.4: XRD patterns of laser irradiated bio-templates at 4, 8, 12, and $26 \mathrm{MHz}$. Enlarged pattern shows emerging peaks, representing of (111),(211), and (221) crystal orientations....... 30 
Figure 3.5: EXD spectrum through LIRS IRS and plain bio-template. A, SEM image of take spectrum. B, EDX Spectrum identifying the presence of Oxygen. 31

Figure 3.6: A, Raman spectra of IRS at $26 \mathrm{MHz}$. B, Raman shifts at 4,8,12, and $26 \mathrm{MHz}$....... 32

Figure 3.7: Shows magnitude of stress component as function of repetition rate. 32

Figure 3.8: Collective cell migration of HeLa and Fibroblast (NIH3T3) in response to functionalized stress component induced on bio-template at different rep.rate are shown on SEM images. The histogram on the right illustrates distance of cell migration corresponding to the magnitude of functionalized stress component. Error bars show SEM; two independent experiments were repeated with $n=3$ in each experiment. Statistical significance is shown with $* \mathrm{p}<0.05, * * \mathrm{p}<0.01$ 34

Figure 3.9: Polar diagrams show, decrease of cell angle by increasing the magnitude of functionalized stress component. A-D, The fluorescent images of Fibroblast (NIH3T3) cell lines seeded on the IRS bio-template with different magnitudes of functionalized stress component, 217.5, 50, -870 and $-1305 \mathrm{MPa}$ respectively. Comparison of images A and D shows that by increasing the functionalized stress component, diversity of cell angle decreases. 35

Figure 3.10: A, Schematic illustration of the distribution of functionalized stress component and cyto-breakage as a result of cell-IRS interaction. B-C, SEM images shows cyto-breakage of Fibroblast (NIH3T3) cells. D-E same phenomena has observed on HeLa cells facing IRS as well. (Arrows and dashed line indicate the side of breakage and the boundary of IRS zone respectively)

Figure 3.11: SEM images show cells protrusion on the side facing IRS. A-B Fibroblast (NIH3T3) cell line, and C-D Hela cell lines. The statistical diagram compares the number of cell protrusions and cyto-breakages on 4,8,12 and $26 \mathrm{MHz}$ for Fibroblast (NIH3T3) and HeLa. Error bars show SEM; two independent experiments were repeated with $n=3$ in each experiment. Statistical 
significance is shown with $* \mathrm{p}<0.05, * * \mathrm{p}<0.01$. (Arrows are indication the lamellipodium protrusion on the SEM images and dashed line indicate the boundary of the IRS zone)............. 36

Figure 3.12: $B$ and D SEM images show the Filopodial extension on the side of the cells opposite to the IRS zone. A and C illustrate the Fibroblast (NIH3T3) cells and HeLa cells respectively on the control samples. Bar graph on the left side (E) comparing the number of filopodia of HeLa and Fibroblat seeded on the IRS bio-template and respective control samples. (Hundred cells were counted for each cell group of samples; error bars denote SD. for each group) 38

Figure 3.13: Fluorescent images of single cell analysis of Fibroblast (NIH3T3) and HeLa cell lines. A and F show single Fibroblast (NIH3T3) and HeLa cell interacting with IRS, arrows indicate both sides of a cell. B and D are example of Fibroblast (NIH3T3) cells seeded on the laser irradiated bio-template at 26 and $4 \mathrm{MHz}$, compared to $\mathrm{C}$ and $\mathrm{E}$ where extension of filopodia and lamellipodia are directional. G and I show the HeLa cells seeded on the laser irradiated biotemplate at 4 and $26 \mathrm{MHz}$ respectively (arrows indicate the cell breakage on side of cell facing the IRS). $\mathrm{H}$ and $\mathrm{J}$ are HeLa cells on the control area. (Optical images showing actin cytoskeleton (green) and nuclei (blue) of cells after 24 hours of culture on IRS bio-template) 39

Figure 4.1: Schematic illustration showing the one-step process of the SNW-plated template. Multiphoton-matter interaction towards the synthesis of the SNW is governed by the laser pulse repetition rate and laser pulse width on which packing index and crystallinity of SNWs plated on the template can be precisely modulated. 50

Figure 4.2: UHR-FE-SEM micrographs in A (a1-a3) illustrate low-packed, medium-packed and high-packed SNWs. HR-TEM images show the Cry-SNW (b1-b3) and Amo-SNW (d1-d3) with respect to their packing index. Size distribution of the constructive component of Cry-and Amo-SNWs plotted in histograms E (e1 and e2), respectively (Statistical analysis done over 1000 particles, with Stan. dev. 0.63). The HR-TEM crystal d-spacing and diffraction patterns demonstrated in image (c1-c2) show the crystallinity of the synthesized SNW. Raman spectra (f1) exhibit the crystallinity changes of the SNW. EDX taken from control and SNW-plated template represent the presence of oxygen because of silica formation (f2 and f3). 52 
Figure 4.3: Cellular response to the crystallinity changes of SNW demonstrated for HeLa and fibroblast cell lines. Interaction of HeLa cells with the Amo-SNW caused an apoptotic-like pathway. Interaction of fibroblasts with both Amo- and Cry-SNWs resulted in a tissue-like structure. Cellular behavior on native (control) substrate showed normal adhesion and proliferation for both HeLa and fibroblast cells.

Figure 4.4: Cellular attraction as a function of packing index of SNWs. SEM and FM of HeLa (A-B) and Fibroblast (C-D) cells, respectively, seeded on Amo-SNW (a1-a3, a5-a7 and c1-c3, c5-c7) and Cry-SNW (b1-b3, b4-b6 and d1-d3, d4-d6) show distinctive attraction characteristics with respect to the packing index of the SNW. Corresponding FM images are also in accordance with SEM micrographs in which attraction of the cells to the SNW increases for highly packed nanowebs. Control samples show random orientation and growth of HeLa (a4) and fibroblast cells (c4). A cell viability assay of HeLa cells (E) shows the increase of dead cells on the AmoSNW along with an increasing packing index. Fibroblast exhibits average of 80 percent cell viability (F). Cytoskeleton to nucleus ratio comparison $(G)$ based on fluorescent imaging revealed the cell shrinkage and nucleus size retraction as HeLa cells interacted with the AmoSNW. Error bars show the standard error of the mean; two independent experiments were repeated with $\mathrm{n}=3$ in each experiment. Statistical significance is shown with $* \mathrm{p}<0.05$, **p $<0.01$ (data presented in graphs are in percentages). 56

Figure 4.5: Statistical analysis based on the cell number per unit area (A) demonstrated that HeLa cells after 24 and 48 hours seeded on low, medium and high packed SNWs for both Amoand Cry-SNW. Cytoskeleton expansion of fibroblast cells (B) at constant cell count and identical unit area show higher attraction of cells to SNWs compare to the control after 24 and 48 hours. Error bars show the standard error of the mean. Two independent experiments were repeated with $\mathrm{n}=3$ in each experiment. Statistical significance is shown with $* \mathrm{p}<0.05$, ** $\mathrm{p}<0.01$ (data presented in graphs are shown as percentages). 57

Figure 4.6: Cellular response to the SNW after 48 hours. SEM micrographs of HeLa cells (AB) and fibroblasts (C-D) after 48 hours of incubation show that the number of HeLa cells on Amo-SNWs (a1-a3) decreased. However, cells on Cry-SNWs (b1-b3) exhibited a proliferative 
behavior. Fibroblast cells showed tissue-like structures on both Amo- and Cry-SNWs (c1-c3, d1-d3). (a4,c4) show HeLa and fibroblast cells seeded on control sample.... 58

Figure 4.7: Demonstration of the interaction of HeLa cells with the SNWs. FM and SEM imaging illustrate that endocytosis of amorphous SNCs is the cause of cell death. The sequence of the apoptosis-induced pathway is also schematically shown. The graph on the top left indicates the efficiency of the induced apoptosis pathway by Amo- and Cry-SNWs. The graph on the left bottom shows the enhancement of cellular attraction as a function of packing index. Error bars show standard error of the mean. Two independent experiments were repeated with $\mathrm{n}=$ 3 in each experiment. Statistical significance is shown with $* \mathrm{p}<0.05$, **p $<0.01$ (data presented in graphs are in percentages). 60

Figure 4.8: HeLa cells in the presence of suspended SNCs (a1-a5); SEM image (a1) and EDX elemental mapping of oxygen (blue) and silicon (green) (a2-a3) and fluorescent imaging (a4a5) represent the SNCs penetrated into the cell (red arrow markers indicate uptake of SNCs by the HeLa cells). HeLa cells on the control sample (b1-b5); SEM imaging and EDX mapping on (b1, b2-b3), where there is no trace of SNCs . Fluorescent image of HeLa cells in the control sample (b4-b5). SEM and FM images of fibroblast cells in presence of suspended SNCs (c1, c4-c5) show enhancement of proliferation behavior compare to the cells on the control (d1, d4d5). EXD analysis of fibroblast in presence of suspended SNCs (c2-c3) compare to the control (d2-d3) do not indicate higher concentration of oxygen and hence uptake of SNCs. (Minimal trace of oxygen in either cell lines are due to substantial nature of organic substance).

Figure 4.9: Cellular interaction with hybrid SNW-plated (Amo and Cry) template exhibited by HeLa and fibroblast cell lines. SEM images of HeLa (A) and fibroblast (B) cells in the presence of hybrid SNWs. HeLa cells exhibited an attraction to the hybrid SNW-plated template; magnified images (a1-a3) show proliferative and apoptotic behavior. Fibroblast cells exhibited an affinity for hybrid SNWs (b1-b3) (dashed lines indicate the boundaries of plated zones). 64

Figure 5.1: Characterization of the PLSN-probes. HR-TEM micrographs illustrating (a1) polyhedron, (a2) intermediate and (a3) polyhedral PLSN-probes. Crystal d-spacing and diffraction 
are demonstrated in images (b1-b3). (C) Size distribution of the fused PLSN-probes plotted in histograms (statistical analysis performed over 1000 random particles with an SD of 0.7). XRD patterns (D) show the dominance of multiple crystal planes upon increased laser fluence. Raman spectroscopy (E) demonstrates the formation of crystalline PLSN-probes. EDX results (F) acquired from the synthesized PLSN-probes do not indicate any compositional changes..... 74

Figure 5.2: Optical properties of the PLSN-probes with respect to crystal plane variation. (A) UV-Vis-NIR spectra of the PLSN-probes exhibit an increase in photon absorption with polyhedral PLSN-probes in the Vis and NIR regions. As the number of crystal planes increases, the absorption increases. (B) Reflectance spectra of the PLSN-probes. The black color spectrum corresponds to the silicon substrate (reference). The PLSN-probes at different ranges of particle size show relatively the same reflection/absorption. The reflectance over the entire UV and NIR band is lowered. PLSN-probes show significantly higher optical absorption, especially in the UV and NIR regions. (C-D) Room-temperature photoluminescence (PL) spectra of the polyhedral PLSN-probes show narrow emission with a significantly large Stokes shift. 75

Figure 5.3: Influence of the multi-crystal planes on PLSN-probes. Irradiation at the visible spectrum (A) shows a stronger excitation of the polyhedral PLSN-probes. Emission of the polyhedral PLSN-probes (B) at excitation wavelengths of 405, 488, 555 and $633 \mathrm{~nm}$ exhibits the multiplex characteristics of the PLSN-probes. Changes in normalized FL intensity due to crystallinity alteration of the PLSN-probes are shown in (C). A comparison of florescence intensity at excitation wavelengths of $405,488,555$, and $633 \mathrm{~nm}$ shows the higher emission intensity of the polyhedral PLSN-probes. 77

Figure 5.4: Cell-selective uptake of the PLSN-probes. Confocal microscopy images of HeLa (A) and fibroblast (B) cell lines without any fluorophores. Image of the cells after incubation with PLSN-probes at 405, 488, 555 and $633 \mathrm{~nm}$ revealed cell-selective uptake. HeLa cells exhibited sequential uptake (a1-a4) through which different layers of the cell, including the cytoskeleton, nucleolemma and nucleus as well as the cell periphery, were screened. Images of fibroblast cells (b1-b4) imply an aversion toward cellular uptake as only the cytoskeleton of the cells could be discerned. Merged images taken at different excitation wavelengths display intercellular details of 
HeLa cells (a5) but only the cell periphery and cytoskeleton of the fibroblast cells (b5). The FL intensity of the multiplex PLSN-probes after 24 and 48 hours of incubation (C) revealed negligible intensity decay. A cell viability assay (D) demonstrated superior biocompatibility of the PLSNprobes after 48 hours on both HeLa and fibroblast cells. 78

Figure 5.5: Intracellular analysis of the HeLa cells. Selective uptake of the PLSN-probes made it possible to visualize intercellular organs of individual cells. From left to right: nuclei, nucleolemma, cytoskeleton and the cell periphery were depicted by internalization of the PLSNprobes. 80

Figure 5.6: PLSN-probes for FL bioimaging. (A-B and C-D) show FL imaging of cells on the control sample and in the presence of PLSN-probes, respectively. The intensity of emitted spectra plotted in (E) illustrates the remarkable FL intensity of the PLSN-probes compared to the control without dyes. 81

Figure 5.7: Studying the health of a cell. (A) PLSN-probes in the size range of 3-5 nm showed an affinity to the cytoskeleton, by which microtubules could be visualized. (B) PLSN-probes ranging from 2-3 nm diffuse through permeable cell membranes and adhere to the nucleus/nucleolemma. Diagnosis of HeLa cells. An FL image (C) shows the overall view of viable and dead cells (dead and healthy cells are indicated by red and yellow arrows, respectively, in the higher-magnification FL image on the right). SEM and FL images demonstrated viable cells (d1 d2) and dead cells (e1, e2). The intensity gradient of the FL images exhibits the dispersion of PLSN-probes within healthy (d3) and dead cells (e3). A comparison of the FL intensities per unit area is shown in (F) and indicates a significantly higher intensity from the dead cells. 82

Figure 5.8: PLSN-probe-assisted FL imaging of HeLa (A) and fibroblast (B) cells. Confocal imaging of HeLa (a1) and fibroblast (b1) cells shows the uptake of the PLSN-probes, as indicated by arrow markers. Cells were stained with FITC and DAPI for both cell lines (a2-a3, b2-b3). Overlay of blue and green emissions (a4, b4). Photobleaching of FITC and DAPI is displayed in (C). 83 
Figure 6.1: Schematic illustration showing the synthesis of the polygonal Si-QPs. TEM micrograph (a) exhibits the polygonal morphology of individual particles. HR-TEM crystal dspacing and diffraction patterns (b-c) demonstrate the crystallinity of the synthesized polygonal Si-QPs. Size distribution plotted in histograms shows 5-20nm range. (Statistical analysis performed over 1000 particles with SD of 0.63). EDX spectrum (d) represent the formation of pure Silicon particles. The UV-Vis spectrum shows higher absorption at longer wavelength. 93

Figure 6.2: Fourier transform infrared (FT-IR) spectroscopy confirms the grafting of amides on the surface of the polygonal Si-QPs. The FT-IR spectra (Figure 6.2) displays as synthesized spectrum (red) of the polygonal Si-QPs, after modification (green) and (blue) the cell culture media. 95

Figure 6.3: Raman mapping of single HeLa cell. (A-C) exhibit the protein, Lipid, and Nucleic acid distribution in the cytoskeleton of a HeLa cell. (D) Shows the overlay of detected substances. SERS activated spectrum of depicted healthy HeLa cell disclose the dominance of Protein, Lipid, and Nucleic acid in different organelle.

Figure 6.4: Raman mapping of apoptotic HeLa cell displays the disintegration of organelle. (AC) exhibit the protein, Lipid, and Nucleic acid distribution in the cytoskeleton of a HeLa cell. (D) Shows the overlay of detected substances. SERS activated spectrum of depicted HeLa cell reveal the dominance of Protein, Lipid, and Nucleic acid in different organelle. 99 Figure 6.5: Statistical analysis shows the ratio of cytoskeleton to nucleus ratio (A). Fluorescence images of fibroblast (B) and HeLa (C) stained with green (FITC) and blue (DAPI). (D) Shows cell viability assessment of HeLa and fibroblast after 24 and 48 hours. The ratio of protein to nucleic acid assessed by Raman spectroscopy (E) shows the nucleus of HeLa increase significantly compared to fibroblast. 100

Figure 6.6: Raman mapping of unstained, unlabelled mammalian fibroblast (NIH3T3) cell displays the disintegration of organelle. (A-C) exhibit the protein, Lipid, and Nucleic acid distribution in the cytoskeleton of a HeLa cell. Overplayed image (D) constructed by merging images (A-C) with color channels. SERS activated spectrum of depicted HeLa cell reveal the 
dominance of Protein, Lipid, and Nucleic acid in different organelle. The sample was irradiated with a light intensity of -- $\mathrm{mW} / \mu \mathrm{m} 2$ at the focal plane in 78 lines of exposure. The exposure time of each line was $180 \mathrm{~s}$, and the images consist of $78 \times 281$ pixels 102

Figure 6.7: Principal component analysis of the data obtained from HeLa and fibroblast cell lines. Scatter plot of HeLa cancer and Fibroblast samples shows differentiation of these cell line based on first three factors of PCA. 103

Figure S1: SEM (A) images show the HeLa cell interaction with suspended Amo-SNSs. Cell roundness is the evidence of cell death induced by endocytosis. The zoom in image shows the porous cytoskeleton of cell that represents the final stage of apoptosis pathway. FM images (B) stained in blue and green distinguish the cytoskeleton and the nucleolus respectively. The penetration of SNWs and their accumulation in the cell body evidenced the cause of cell death.

Figure S2: Characterization of the hybrid SWNs evidences the simultaneous presence of crystalline and amorphous orientation. UHR-FE-SEM micrograph of the Hybrid SNWs shows the morphological structure of as-synthesized SNWs (A). HR-TEM image used for particle size measurement (B) shows self-assembled SNWs. Diffraction patterns (C-D) taken by HR-TEM evidence the formation of Cry- and Amo-SWNs. Size distribution histogram (E) show an average size of $12 \pm 0.5 \mathrm{~nm}$ (Statistical analysis done over 1000 particles count).............. 114 Figure S3: Hybrid Silica Nano-web composes of crystalline and amorphous was synthesized at intermediate laser pulse width of 714 fs. The FM and SEM images (A and B) showed that efficiency of this nanostructure to attract cells has remained same, regardless of the crystallinity of SNWs. However, HeLa cells have shown rounded and ruptured cytoskeleton. The number of dead cells is not comparable with Amo-SNWs. 114 


\section{LIST OF ABBREVIATIONS}

Amorphous (Amo)

Crystalline (Cry)

Carbon materials (SWCNTs)

Cadmium selenide (CdSe)

Chemical vapor deposition (CVD)

Centre for Nanostructure Imaging (CNI)

Extracellular matrix (ECM)

Energy Dispersive X-ray (EDX)

Electron Backscatter Diffraction (EBSD)

Energy-dispersive X-ray spectroscopy (EXD)

Fluorescence (FL)

Fluorescence microscopy (FM)

Field emission scanning electron microscope (FE-SEM)

Heat affected zone (HAZ)

HR-TEM diffraction pattern (FFT)

High-resolution transmission electron microscope (HR-TEM)

Induced Residual Stress (IRS)

Laser chemical vapor deposition (LCVD)

Megahertz (MHz)

Near-infrared (NIR)

Near-ultraviolet (NUV)

Nanoparticles (NPs)

Natural Science and Engineering Research Council of Canada (NSERC)

Photoluminescence (PL)

Transmission electron microscopy (TEM)

Quantum dots (QDs)

Repetition rate (rep.rate)

Silicon (Si)

Slica nano-web (SNW) 


\section{Si-based QDs (Si-QDs)}

Silica nanoclusters (SNCs)

Silicon nanoparticles (SNPs)

Scanning electron microscopy (SEM)

Self-assembled silica nano-webs (SNWs)

Semiconductor quantum dots (silicon-based QDs)

Three-dimensional (3D)

Ultrashort Pulsed Laser (USPL)

Ultraviolet-visible-near-infrared (UV-Vis-NIR)

X-ray Diffraction (XRD) 


\section{CHAPTER 1}

\section{INTRODUCTION}

\subsection{Silicon as an ideal biocompatible materials}

Silicon is known to be the second most abundant element on the earth's crust after oxygen, supplying rich and low-cost resources for a myriad of silicon-based applications. By virtue of its excellent semiconductor and mechanical properties, silicon is the leading semiconductor materials and is dominating the electronics industry. Notably, novel structural, optical or/and electronic characters emerge when the dimensions of silicon materials are reduced to nanoscale level. In the last 20 years, we have witnessed the enormous advancement in fabricating silicon nanomaterials and the rapid development of silicon nanomaterials-based applications in various fields including electronics, energy, environment, biology, and biomedicine. Taking advantage of nontoxicity of silicon, exploration of silicon nanotechnology for biological and biomedical applications is of particular interest.

\subsection{Silicon based nanomaterials for biological applications}

Nanomaterials have drawn great attention in different aspects due to their unique physical and chemical characteristics. In the realm of biology and biotechnology, utilization of nanomaterials in particular nanoparticles have revolutionized the frontiers of future nano-bio-medicines. Biocompatible nanoparticles, for instance, have shown the capability of being used as targeted therapy which can not only be an alternative for current chemotherapy but also have higher efficiency of treatment- Apart from therapeutic applications of nanomaterials in biology, nanoparticles assisted imaging has currently exhibited promising diagnostic applications by which drawbacks of the common methods such as fluorescent dyes could be overcome. Additionally, nanoparticles have numerous potential applications in the fabrication of nano-devices in microelectronic, biomedical, photonic fields, tissue engineering, and filtration. 


\subsubsection{Nanostructured materials for tissue engineering}

The employment of nanostructures in biological usage is exclusively limited to extracellular matrix (ECM). Considering that, cells are inherently sensitive to local signals from chemical and physical stimuli, such as rigidity, smoothness, wettability, surface molecular structure, and surface charge; topographical changes of cell-biomaterial interface lead to advancements of cell patterning, cell isolation, and collective cell migration. Extra Cellular Matrix (ECM) therefore have also been implemented to control the size, shape, and differentiation of cells, as well as cell-cell contacts. Along with the development of micro/nano-fabrication, engineering of cell behavior has been made possible via design of complex three-dimensional (3D) nanostructure with spatiotemporal chemical/physical attributions.

In addition, micro/nano-structures such as nanofibers have been applied for cell culture and scaffolds. These extensive studies benefit the understanding of fundamental cell behaviors and cell-material/cell-microenvironment interactions and promote the development of tissueengineering materials for advanced biomedical implants and devices. Hence, the importance of understanding chemical/physical aspect of nano-bio interface interactions as an essential tool for biological studies and applications has progressively increased. That is because 3D nanostructures are an important part of cell microenvironments, as shown in the nanoscaled villi and podia of cell surfaces and the nanofibers of ECM. Moreover, some important biological studies in recent years, such as cancer diagnosis and therapeutic, have raised requirements for precisely nanoscaled control of cell-material interactions. These factors all together promote the creation of more advanced and smart bio interfaces at the 3D nanoscale. Thus far, Extensive studies underline the capabilities of nanoscale structured temples for tissue engineering, directing and controlling cell growth as examples of biological applications.

\subsubsection{Conventional trends on synthesis of Silicon nano materilas}

Among various techniques of synthesising nanomaterials such as electrospinning, phase separation, self-assembly processes, thin film deposition, chemical vapor deposition (CVD), laser ablation and laser chemical vapor deposition (LCVD), ultrashort pulsed laser ablation has demonstrated an extraordinary capability to precisely modulate size, shape, as well as physical and chemical attributions of the synthesised nanomaterials. Ultrafast laser ablation is a promising technique to generate nanostructures due to its simplicity in equipment configuration, high speed, 
and low investment. Ultrafast lasers have been extensively utilized over the last decade and its rapid development has opened a wide range of new applications in numerous areas such as analytic sampling, thin film deposition, surface micromachining, MEMS and NEMS fabrication, laser nanofabrication, and biomedical applications. Ultrashort lasers have an ability to generate very high peak intensity, resulting in rapid deposition of energy into the material.

The pulse duration in the Ultrashort laser interaction with a solid target appears to be shorter than all distinctive relaxation times: the electron heat conduction time, the electron to ion energy transfer time and consequently the hydrodynamic or, the expansion time. The most important advantage of Ultrashort laser ablation over other pulsed lasers is that there is little or no collateral damage in dielectric materials, and reduced heat affected zone (HAZ) in metals due to shock waves and heat conduction produced in the material being processed. Furthermore, plasma shielding of the incoming laser beam with ablated material can be prevented in Ultrashort lasers which can lead to maximum absorption efficiency. In the following sections, silicon-based nanostructures synthesized by ultrashort pulsed laser in the range of femtosecond in respect to therapeutic and diagnostic applications are discussed.
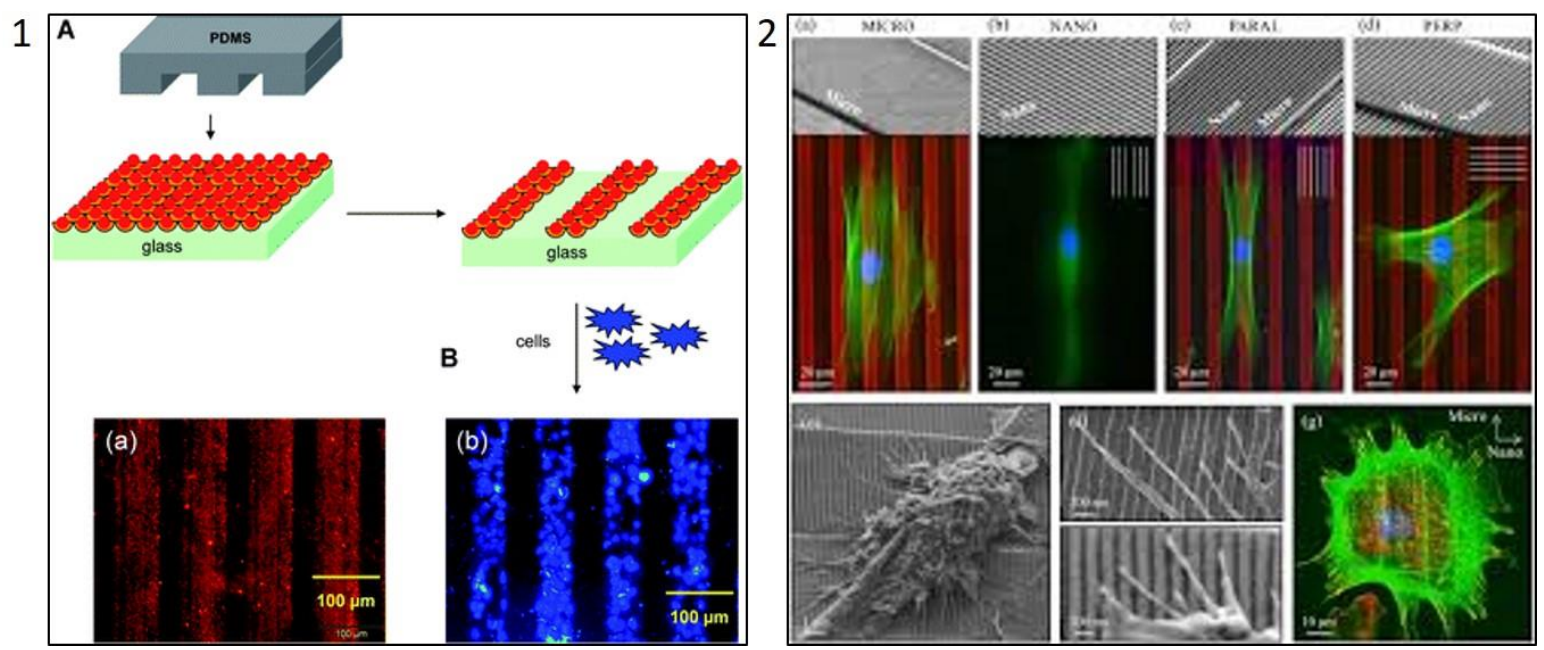

Figure 1.1: Schematic of cell pattering by means of micro-stamp (1) and micro-grooves (2) (Courtesy [chapter3 1-12]) 


\subsection{Regulation of cellular behavior}

Cell patterning is defined as a process to position cells on a substrate and/ or regulating the fate of cells, especially on a biocommatible chip, with specific spatial selection to keep stable cell growth on the substrate. Preferred pattering or arraying of cells is essential for cellular or tissue function. Cell pattering has a wide range of applicartions such as tissue engineering, wound healing, biosensing and lab-on-chip and study of cell migration.
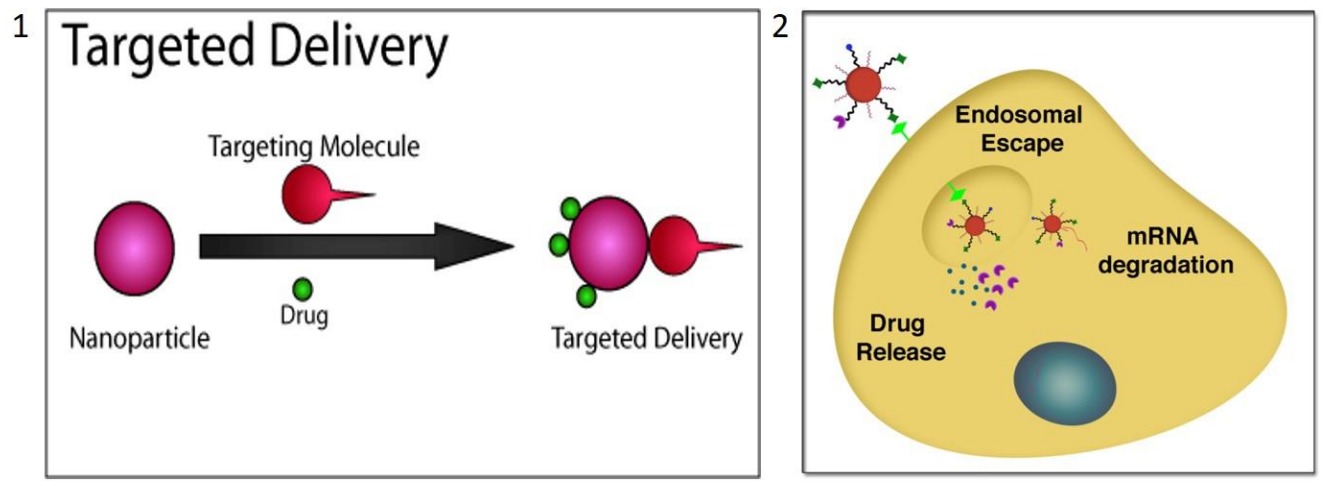

Figure 1.2: Demonstration of using nanoparticles as drug carrier (1). Interaction of tagged nanocarrier with the targeted cell (2). (Courtesy [chapter4 5-21])

Figure 1.1 shows a schematic depiction of the micro-stamp and Raman micro-grooves for cell pattering. Early investigations for cell patterning reported in 1910 studied the interaction between embryonic cells and natural webs. Over the years, other synthetic microstructuces used for cell patterning have been reported. These structuces mostly are reliant on grooved structures, microstamp and grids. Another approach is to use chemical stimulies. Surface coatings of a protein monolayer, for instance, has been commonly implemented to guide cell growth. These coatings either promote or inhibit cell adhesion to define a cell pattern. However, the suitable competent to be deposited is not trivial for cells and is dependent on cell type. Therefore studies of biocompatible polymers, extracellular matrix protein, and new coating methods have regularly been reported. For all above mentioned approaches, an artificial pattern transferrd onto the substrate of cultured cells is essential and commonly accomplished by photolithography. However, an alternative patterning approach that modulate the cell fate without actively confining the cells is required. Hence, a novel concept of using femtosecond laser to precisely induce functionalized stress components onto the bio-template to modulate cells behavior has been investigated (as given 
in chapter 2). The study of cell-substrate interaction and successful regulation of cells migration and directionally has led to better understanding the role of Silicon substrate used as a template for therapeutic and diagnosis.

\subsection{Silicon Based Nanostructures for Cancer Therapy}

In the past decade, there has been vast advancement of silicon nanotechnology, which provides exciting avenues for biological, and biomedical applications. Among them, the exploration of silicon nanotechnology for bioapplications (so-called silicon nano-biotechnology) is one of the most important branches, receiving extensive attention and revolutionizing basic research and clinical applications in recent years. Silicon nanoparticles have established as excellent candidates for medical applications due to their superior biocompatibility and high surface area in nanoscale. However, as shown in Figure 1.2(1,2) the use of silicon nanoparticles for cancer therapy relies greatly on acting as logistic for drug delivery. Moreover, metallic nanoparticles have been commonly used for targeting cancer treatment. However, metal nanoparticles present biocompatibility issues. Despite acting as an accurate drug carrier to a target such as a group of cancer cells, there are negative side effects such as kidney and liver damage due to degradation of metallic nanoparticles. When nanoparticles reach the bloodstream, they can get stuck in internal organs, and after a while they begin to harm the organism due to prolonged toxic effects. The new findings revealed/ reveals that silicon based nanomaterials not only are biocompatible but can even provide a health benefit. When these silicon nanoparticles dissolve, they become silicic acid, which is a vital substance for strong bones and healthy connective tissues.

Therefore, based on the previous studies of scientists worldwide and recent progressions of our group, this dissertation aims to introduce silicon based nanostructure for biological and biomedical applications, particularly for cancer therapy. In quest of synthesis of multifunctional bio-template for cancer therapy, the influence of polymorphic Silica Nano-web on mammalian Fibroblast and cancer HeLa cell lines have been investigated (presented in chapter 3). The objective of crystallinity alteration of the synthesized silica was to create selective functionality by which cancer and mammalian cells were treated distinctively. Moreover, in order to enhance the efficiency of synthesized bio-template, the attractive characteristic of template being used for scaffold has been resembled. Additionally, the therapeutic attribution of this bio-template were 
inherited from silica nanoparticles that are self-assembled to form 3-D Nano-web. It is worthwhile to point out that, a method by which atomic alteration of silicon nanostructures results in selectively target a cell of interest has not been reported.

\subsection{Silicon Nano-Probes for Diagnostic Bioimaging}

Research into quantum dots (QDs) is an intriguing branch of science. The unique characteristic of QDs is that they are nanocrystal semiconductors. This means that under certain conditions the QDs can convert energy into specific wavelengths on the electromagnetic spectrum (Figure 1.3(1)).
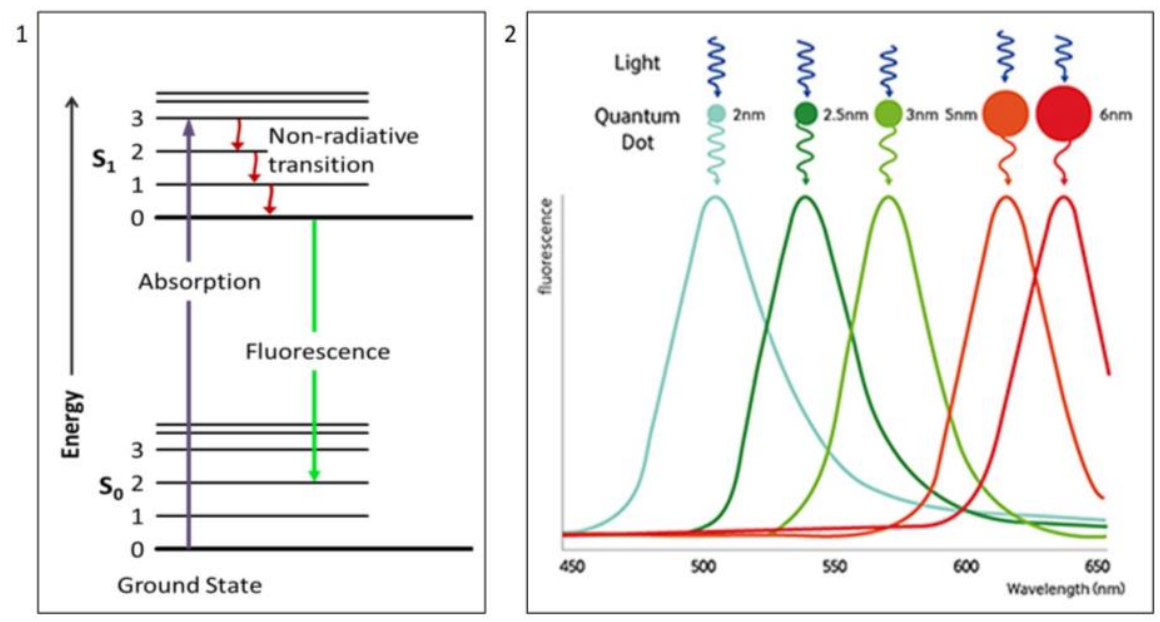

Figure 1.3: Scheme (1) illustrates the relation between the energy level and processes of exciton creation. (2) Exhibits the size dependency of quantum dots on the emitted light (Courtesy [chapter5 12-22])

The colour that the QDs produce is primarily dependent on the size (2 to 10 nanometers) and shape as demonstrated in Figure 1.3 (2). Furthermore, QDs near 10nm will emit red light, while QDs near $2 \mathrm{~nm}$ will emit blue light. The conceptual operation of the QDs is theorized by the quantum confinement effect which occurs when the nanocrystal is smaller than the Bohr radius. The quantum confinement effect can be interpreted as nanocrystals being compressed to smaller than normal. This effect is noticed when QDs are excited, as they produce different colours with respect to their shape and size. Other characteristics of QDs are as follows: geometries are inclusive but not restricted to nanoparticles/cubes/spheres/tubes/carpets/dots/rods, adjustable morphology and 
countless combinations of elements. A few examples of QD molecules include silicon, carbon and cadmium sulfide. Quantum dots have favorable advantages compared to previous methods, which include reduction in photobleaching, quantum yield and a vast range of applications. These applications include LCD displays, lights, sensors, and photovoltaics. There are numerous different methods to synthesize QDs with respect to laser ablation. Silicon QDs have been receiving notable attention due to their reputable abilities as an electronic semiconductor material $[2]$.

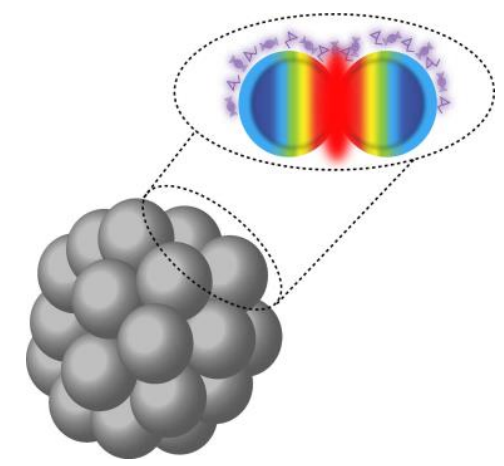

Figure 1.4: Scheme illustration of SERS-enabled Si probe by localized surface plasmon

The advantages of silicon QDs also include the following: economic viability, substantial toxin reduction when compared with heavy metal QDs, large quantity, the ability to produce infrared light, and gradual heat loss to carriers. Silicon QDs can be synthesized near-infrared (NIR) or near-ultraviolet (NUV) in the electromagnetic spectrum. To produce NIR silicon QDs, laser ablation of the silicon must be done in a gaseous atmosphere. Conversely, NUV is created through the process of laser ablation in an aqueous environment. Advancement in biotechnology is demanding for substitution of current fluorescent dyes due to their lack of versatility. The application of conventional fluorophores is limited to the specific wavelength of light and their efficiency is subjected to intensity of light. Photo-bleaching for instance is the other drawback of fluorescent dyes. Crystalline silicon in sub-nano range synthesized by laser-matter interaction has shown to be a feasible replacement for the current fluorescent agents. Moreover, it demonstrated that sub-nano silicon particles can not only be used for bioimaging but they can also be utilized for diagnostic purposes. Therefore, simultaneous benefits of bioimaging and diagnostic can be achieved by alteration of physical and chemical properties of silicon particles in subnano scale. 


\subsection{Silicon Quantum Probe towards Cancer Diagnosis}

Raman spectroscopy is a powerful technique for detection of chemical and bio-compounds but it suffers from a major drawback of low intensity that is required to detect these analytes at the ultrahigh sensitivity. However, this drawback can be tackled by inducing the Surface enhanced Raman Scattering (SERS) in which the analyte signal can become greatly enhanced. Currently noble materials such as gold and silver are being used as SERS-active platforms for analytical detection. The recent studies done in our Lab have shown that semiconductor materials also have the ability to exhibit SERS characteristics that can be used as an alternative to noble metals. However, the current state of using SERS-active platforms can only be exploited for chemical sensing. The 5th chapter of this dissertation presents the synthesis of polygonal silicon nanoprobes though a multi-photon ionization mechanism that can be used for in-situ live cell Raman spectroscopy. The unique characteristic of Si probes relies on localized surface plasmon (LSP) introduced by polygonal morphology which enables the SERS (Figure 1.4). The other advantage of the synthesis is that quantum size of this probe along with an affinity to functional groups has overcome the limitation of cell internalization.

\subsection{Summary of the current limitations}

The current application of nanoparticles in the field of biotechnology is limited to take advantage them as a drug carrier, where they play role as logistic carrier for duped drug. However, the nanoparticles can be tuned to act as a drug. Since there are limited studies surrounding this issue, this thesis aimed to investigate the possibility of utilizing Si-based nanomaterials for therapeutic and diagnostic applications.

Additionally, early cancer diagnosis is essential for cancer therapy and successful treatment. Hence, there is an increasing interest in developing and implementing more efficient and accurate methods to diagnose. One of the conventional methods of cancer diagnosis is sampling and cell culture. Although this method is well-established, it is not time efficient and also cannot be implemented as so called in-situ. 
These limitations of the conventional therapy and diagnosis are the motivation behind this thesis. The contents of this thesis aims to take advantage of multidisciplinary methods such as multiplex photoluminescence and Raman spectroscopy for cancer therapy and diagnosis.

\subsection{Research Objective}

Si-based nanostructures/particles synthesized through ultrafast pulsed laser ionization offer unique physical and chemical characteristics. This fabrication technique is able to generate a wide range of shape, size with precise control on physicochemical properties. This controllability on the synthesized materials has been explored to be used for different objectives towards therapeutic and diagnostic of cancer HeLa and mammalian Fibroblast cell lines. The main objectives of this research fall in the following studies:

\subsubsection{To determine the interaction of cell-matter and modulation of cellular behavior}

The new concept of cell pattering without means of external stimulus has been investigated. The goal is to achieve a method by which cell regulation is based on cellular perception.

\subsubsection{To synthesize polymorphic 3-D silica nanostructures}

To synthesize crystalline and amorphous 3-D silica Nano-structure at different packing index. Varying the laser pulse width showed that polymorphic silica Nano-structure can be synthesized while pulse repetition rate has an influence on the density (packing index) of the deposited Nanostructure.

\subsubsection{To generate a multiplex PhotoLuminescent probe for cell-imaging and diagnosis}

To investigate the influential multiplane crystallininity of Silicon nanoparticles on efficiency of PhotoLuminesce property. Silicon-based Nanoparticles consist of multiple crystal structures generated with respect to cell-imaging and diagnosis.

\subsubsection{Fabrication of Raman sensitive probe for cancer diagnosis}


To study the cellular behavior, diagnose cancerous cell lines and fabricate SERS-active probes. Ultrashort pulsed laser was utilized to fabricate the probes within quantum scale. In this study, the role of quantum scale si-based probes on cencer detection and cell difrentiation was investigated.

\subsection{Organization of thesis}

This thesis has been organized in a manuscript based manner. The following is the list of manuscripts published in previewed scientific journals, as presented in each chapter.

Chapter-2 Summarizes the experiments and instrumentations used to conduct this thesis.

Chapter-3 Describes the role of induced stress components on cell behavior. The new concept of using residual stress to remotely signal cell fate has established and examined on two separate cell lines; cancerous HeLa (with aggressive proliferation characteristic) and mammalian fibroblast that has a tendency to form tissue. Our finding revealed the minimum stress required for cell response, defined as threshold of cytocompatibility. Studding the cell matter interaction has proven the biocompatibility of the substrate and helped to design more efficient platform for therapeutic applications.

Chapter-4 Demonstrates the therapeutic application of the silicon based 3D nanostructures. The developed Self-assembled Silica Nano-webs has an advantage of acting as extracellular matrix (ECM) and nanoparticles concurrently. These unique properties lead to cell attractions and selective therapy by which the HeLa cancer cells undergo an intensified apoptotic pathway whereas fibroblast cells exhibit tissue structures. The selectivity functions of Silicon nanoscale materials have been extended to be used for diagnosis purposes which is further investigated in the next chapter.

Chapter-5 Introduces a label-free multiplex photoluminescent probes as a potential substitute for quantum dots in bioimaging with added value of potential as a diagnosis agent. The silicon nanoprobes exhibited cell-selective uptake that allows the differentiation and diagnosis of HeLa and fibroblast cells. Moreover, the synthesized nano-probes showed supremacy in compare to conventional organic dyes for cell staining. Although the diagnosis application of them are the prior objective of this study, the results of this chapter implied the idea of using quantumized 
silicon particles for extended cell differentiation and diagnosis with an overlook on clinical applications.

Chapter-6 Delineates the synthesis and SERS-activation of quantum-scale silicon probes for cancer diagnosis. Polytonality of the synthesized Silicon quantum probes enhances the Raman spectrum of cellular components. The current methods to enhance Raman scattering relies on using platforms which cannot be implemented for intercellular (in-situ) analysis. This study shows the capability of the polygonal Silicon quantum probe to internalize the targeted cells and be used to monitor the chemical changes of live-cells. The results have given insight to differentiation and diagnosis of cancer cells.

Chapter-7 Summarizes the discussion in regards to manufacturing and synthesizes of the Silicon based nanomaterials as well as the results of these studies, and proposals for the future direction of this research. 


\section{CHAPTER 2}

\section{EXPERIMENTAL AND METHODS}

\subsection{Materials and methods}

The substrates used in this thesis are high-quality polished single crystal $\mathrm{N}$-type silicon wafers with crystallographic orientation of (100) and $625 \mu \mathrm{m} \pm 25 \mu \mathrm{m}$ thickness. Supplied by University Wafer. Prior to Laser irradiation the silicon wafer diced into $2 \mathrm{~cm}^{2}$ square-shape samples. In order to remove the debris and residues, the silicon ships were ultrasonically cleaned in a 50 degree Celsius acetone bath for 15 minutes, followed by rinsing and drying.

\subsubsection{Experimental setup}

Based on the required properties of the nanomaterials, the laser parameters were precisely tuned. An ultrashort pulsed laser (USPL) in femtosecond range was utilized; (Yb-doped femtosecond laser beam (Clark-MXR, Inc.; IMPULSE Series ultrashort pulse laser)). This laser system is capable of producing central wavelengths of $1040 \mathrm{~nm}$ and a laser pulse width varying from $214 \mathrm{fs}$ to $1428 \mathrm{fs}$. As well as, the laser pulse repetition rate is tuneable between $4 \mathrm{MHz}$ (Low rep.rate) and $26 \mathrm{MHz}$ (High rep.rate). The maximum operating average power is $16 \mathrm{~W}$. Moreover, a collateral damage caused by laser-matter interaction is minimal or negligible in the implemented laser. The utilized laser setup in this study was also equipped with a computerized Galvano scanner to precisely implement the synthesis processes.

\subsubsection{Laser induced functionalized stress component}

To precisely functionalize the stress component on the IRS, a pulse repetition rate of 4,8,12 and $26 \mathrm{MHz}$, and scanning velocity of $10 \mathrm{~mm} \mathrm{~s}^{-1}$ were implemented. Laser pulse width and average power were set up and remained constant at 214fs and $16 \mathrm{~W}$ respectively. The laser spot-size at the

focal point was adjusted to $10 \mu \mathrm{m}$ diameter after manipulation by a pair of galvanometers through 
optics lenses. Prior to seeding the cells, the sample was irradiated at the focal point by a computerized Galvano scanner to precisely implement and IRS biomimetic architecture.

\subsubsection{Synthesis of Silicon Nano web}

The self-assembled silica nanostructures were synthesized by a single-step femtosecond laser processing of silicon substrate. For the purpose of this study, we fabricated the self-assembled silica nanostructures at pulse widths of 214, 714 and 1428 fs and repetition rates of 4, 12 and $26 \mathrm{MHz}$ with laser fluence of $1.57,3.39$ and $10.18 \mathrm{~J} / \mathrm{cm}^{2}$ in order to achieve high, medium and low packing indices of synthesized nanostructures, respectively (laser power of $10 \mathrm{~W}$ and Gaussian $1 / \mathrm{e}^{2}$ beam diameter of $\left.10 \mu \mathrm{m}\right)$. Scanning speed was constant at $2 \mathrm{~mm} / \mathrm{s}$.

\subsubsection{Synthesis of photoluminescence silicon nano probes}

The self-assembled PLSN-probes were synthesized by single-step femtosecond laser processing of the silicon substrate. For the purpose of this study, we fabricated the polyhedron, intermediate and polyhedral PLSN-probes with a pulse width of $214 \mathrm{fs}$ and repetition rates of 4, 12 and $26 \mathrm{MHz}$, respectively. The laser fluences at 4, 12 and $26 \mathrm{MHz}$ were measured as $1.57,3.39$ and $10.18 \mathrm{~J} / \mathrm{cm}^{2}$, respectively. (The laser power was $16 \mathrm{~W}$, with a Gaussian $1 / \mathrm{e}^{2}$ beam diameter of $10 \mu \mathrm{m}$, and the scanning speed was $2 \mathrm{~mm} / \mathrm{s}$; all parameters were held constant.)

\subsubsection{Synthesis of the SERS-enabled polygonal Si-QPs}

The sequential multiphoton ionization was initiated by laser irradiation of single crystalline Silicon chip. For the purpose of this study; average laser power, repitation rate (rep.rate) and laser pulse width has set to $16 \mathrm{~W}, 26 \mathrm{MHz}$ and $214 \mathrm{fs}$. An inert atmosphere condition applied to prevent oxidation of the synthesized Si-QPs. Utilizing the pulsed laser (USPL) is advantageous since the absorption of multiphoton excitation energy overcomes the ionization threshold of the Silicon chips. Moreover, given the localized laser beam spot $(10 \mu \mathrm{m})$ within the focal point, results in precise control over the synthesized Si-QPs. 


\subsection{Cell culture and seeding}

In order to remove all debris and impurities, samples were ultrasonically cleaned followed by exposer to the UV light for 20 minutes prior to seeding the cells. Laser treated bio-templates were rinsed with Phosphate Buffered Saline (PBS) (1X strength compose of: $138 \mathrm{mM} \mathrm{NaCl}, 2.7$ $\mathrm{mM} \mathrm{KCl}, 1.9 \mathrm{mM} \mathrm{NaH}_{2} \mathrm{PO}_{4}, 8.1 \mathrm{mM} \mathrm{Na} 2 \mathrm{HPO} 4$ ) before seeding the cells then dried and sterilized under UV light for 20 minutes. Fibroblasts cell lines (NIH3T3) and human cervical cancer cell lines (HeLa, ATCC, American Type Culture Collection, and ATCC No. CCL-2) were employed in cell experiments to ascertain comparative cell controllability of mammalian and cancer cell lines. Fibroblast (NIH3T3) cells were grown in a DMEM medium containing 10\% heat activated fetal bovine serum with $1 \%$ penicillin-streptomycin antibiotics (Pen-strep). HeLa cells were grown in a DMEM-F12 medium supplemented with $10 \%$ fetal bovine serum and $1 \%$ Pen-strep. Subsequently, the cells were separately cultured on the substrates that are placed in petri dishes with seeding density of 750,000 cells $/ \mathrm{cm}^{2}$ of substrate surface area. The petri dishes were placed in an incubator for 24 hours at $37^{\circ} \mathrm{C}$ temperature.

\subsubsection{Cell imaging and morphology}

For morphological study of cells, after the prescribed time period, spent medium were aspirated. This was followed by the fixing of samples in $2 \%$ glutaraldehyde in $0.1 \mathrm{M} \mathrm{pH} 7.3$ sodium cacodylate buffer for an hour. Next, the samples were immersed in $0.1 \mathrm{M}$ sodium cacodylate buffer with $0.2 \mathrm{M} \mathrm{pH} 7.3$ sucrose for 20 minutes. Preceded by dehydration as an increasing concentrations of alcohol for 20 minutes. The samples were then dried to a critical point. At the conclusion of the experiment, cells were ready to be directly observed using Scanning Electron Microscopy (SEM). Images were acquired at $100 \mathrm{x}$ to $10,000 \mathrm{x}$ magnification with and accelerating voltage of $10 \mathrm{kV}$, and a probe current of 50 to $60 \mu \mathrm{A}$. 


\subsection{Instrumentation}

\subsubsection{Electron backscattered diffraction (EBSD)}

The crystal orientation of the sample of interest was determined using EBSD. Oxford Instruments INCA interfaced to a Hitachi SU3500 SEM with a LaB6 electron gun. These systems can automatically obtain a crystal orientation map by scanning a rectangular area on the surface of the specimen, which is titled $70^{\circ} \mathrm{C}$ from the horizontal.

\subsubsection{X-ray diffraction (XRD)}

The elemental composition and crystal structure of the samples was characterized using Rigaku MiniFlex 600 Benchtop X-ray diffraction (XRD) under $\mathrm{Cu}-\mathrm{K} \alpha$ radiation $(\lambda=0.154 \mathrm{~nm})$.

\subsubsection{Energy - dispersive X-ray spectroscopy (EDX)}

To identify possible compositional changes on the synthesized nanomaterials, elemental analysis was performed. The advantage of employing SEM/EDX lies in shallow penetration depth of the interactive electron beam in constract to XRD. Thus, surface elemental analysis can be achieved.

\subsubsection{Micro-Raman spectroscopy $(\mu \mathrm{RS})$}

Micro-Raman spectroscopy was utilized to measure and characterize the types of stresses (Tensile / Compressive) within a small measurement spot size, below $5 \mu \mathrm{m}$ in diameter, and a submicron spatial resolution. Moreover, depending on the experimental setup Micro-Raman spectroscopy was used for studying the chemical composition. Raman spectra were obtained using a Bruker SENTERRA, Dispersive Raman Microscope having a central wavelength of $532 \mathrm{~nm}$ as an excitation source. 


\subsubsection{Cell imaging and morphology}

The cellular morphology of cells seeded on samples was observed using a Hitachi SU1510 scanning electron microscope. After the prescribed time period, spent media were aspirated. This was followed by the fixing of samples in $2 \%$ glutaraldehyde in $0.1 \mathrm{M} \mathrm{pH} 7.3$ sodium cacodylate buffer for 1 hour. Next, the samples were immersed in $0.1 \mathrm{M}$ sodium cacodylate buffer with $0.2 \mathrm{M} \mathrm{pH} 7.3$ sucrose for 20 minutes, followed by dehydration at increasing concentrations of alcohol for 20 minutes. The samples were then critical point dried and coated by gold, at this point cells were ready to be directly observed using SEM.

\subsubsection{Fluorescence microscopy}

To conduct fluorescent visualization the samples are first fixed in methanol-free paraformaldehyde followed by incubation in skim milk to prevent non-specific binding. Actin and cytoskeleton are stained by incubation of the samples with phalloidin-Alexa Fluor 488 (Life Technologies) followed by DAPI (4', 6'-diamidino-2-phenylindole, Life Technologies) to stain the nucleus. The samples are studied using an epi-fluorescent Nikon E-400 microscope (Nikon, Canada). Seeded cells were also fluorescence-imaged on a Zeiss LSM 710 META upright confocal laser-scanning microscope using 60× magnification water-dipping lenses for the monolayer cultures. Wavelengths of 405, 488, 555 and $633 \mathrm{~nm}$ were used. Image data acquisition and processing were performed using a Zeiss LSM Image Browser, Zeiss LSM Image Expert and ImageJ.

\subsubsection{Morphology and physicochemical characterization}

Ultra-high resolution Field emission SEM (UHR-FE-SEM) and energy-dispersive X-ray spectroscopy (EDX) (Hitachi SU8200) were employed to characterize the surface morphology

and element analysis of the self-assembled silica nanostructures deposited on the samples. Nanoclusters of nanoparticles were examined in terms of crystallinity and particle size distribution using high-resolution transmission electron microscopy (HR-TEM) (JEOL JEM2010). To separate the nanostructures from the templates for HR-TEM analysis, we immersed the samples in an isopropyl alcohol solution and ultrasonically vibrated the solutions. A drop 
of the solution was then placed on a grid and dried in a desiccator. (The overall number of particles counted to plot the size distribution histogram were over 1000 particles).To characterize the crystallinity of silica clusters, the diffraction pattern was obtained. An ultra-high-resolution scanning electron microscope was also employed for the morphological characterization and to determine the packing index of nanostructures made of self-assembled silica clusters. Packing index was defined as nanoparticle size distribution and nanogap size distribution for each ablation area. Analysis of images obtained from HR-TEM and HR-SEM, using image processing software ImageJ, revealed three classifications of low, medium, and high structural packing indices. Further elemental analytical techniques were employed using Oxford EDX to identify the elemental changes as a result of silicon oxidation. Moreover, this technique was exploited to characterize the SNC uptakes caused by endocytosis. Compression of oxygen stoichiometry of cells on controls with cells in the presence of SNCs determined by elemental mapping elucidated the apoptotic behavior of the HeLa cell line. 


\section{CHAPTER 3}

\section{FUNCTIONALIZED STRESS COMPONENT ONTO BIO-TEMPLATE AS A PATHWAY OF CYTOCOMPATIBILITY}

Keshavarz, M.; Tan, B.; Venkatakrishnan, K. "Functionalized Stress Component onto Biotemplate as a Pathway of Cytocompatibility." Scientific Reports 2016, 6, 35425.

\subsection{Abstract}

This in-vitro study introduces residual stress as a third dimension of cell stimulus to modulate the interaction between cells and bio-template, without the addition of either chemical or physical stimuli onto the bio-template surface. Ultrashort Pulsed Laser (USPL) irradiation of silicon-based bio-template causes recrystallization of silicon, which mismatches the original crystal orientation of the virgin silicon. Consequently, subsurface Induced Residual Stress (IRS) is generated. The IRS components demonstrated a strong cytocompatibility, whereas the peripheral of IRS, which is the interface between the IRS component and the virgin silicon surface, a significant directional cell alignment was observed. Fibroblast cells showed to be more sensitive to the stress component than Hela cancer cells. It revealed that cytocompatibility in terms of cell migration and directional cell alignment is directly proportional to the level of the IRS component. Higher stress level results in more cell alignment and border migration width. There is a stress threshold below which the stress component completely loses the functionality. These results pointed to a functionalized biotemplate with tunable cytocompatibility. This study may lead to a new tool for the designing and engineering of bio-template.

\subsection{Introduction}

There is an ample amount of literature surrounding the needs for modulation of cell behavior for bio-applications, which indicates that study of cell interaction with biomaterials/bio-templates is 
of great importance for controlling cell behavior, growth, and migration. With advancement in Nano and Microscale technologies, a variety of approaches have been developed to engineer invitro cell interaction, including topographical patterning, changing surface chemistry, mechanical loading (stiffness), and the combinations of these methods ${ }^{1}$. Micro and nano-topographic patterns is a popular tool for directional migration of cells. This method mechanically guides the cell migration by restricting cell mobility in channels and grooves ${ }^{1-6}$. Lithography, for instance, is one of the methods to create microchannels with an acceptable feature resolution ${ }^{7}$. The major challenge of this approach is that the fabricated topographic pattern does not represent the true extra cell matrix in nature.

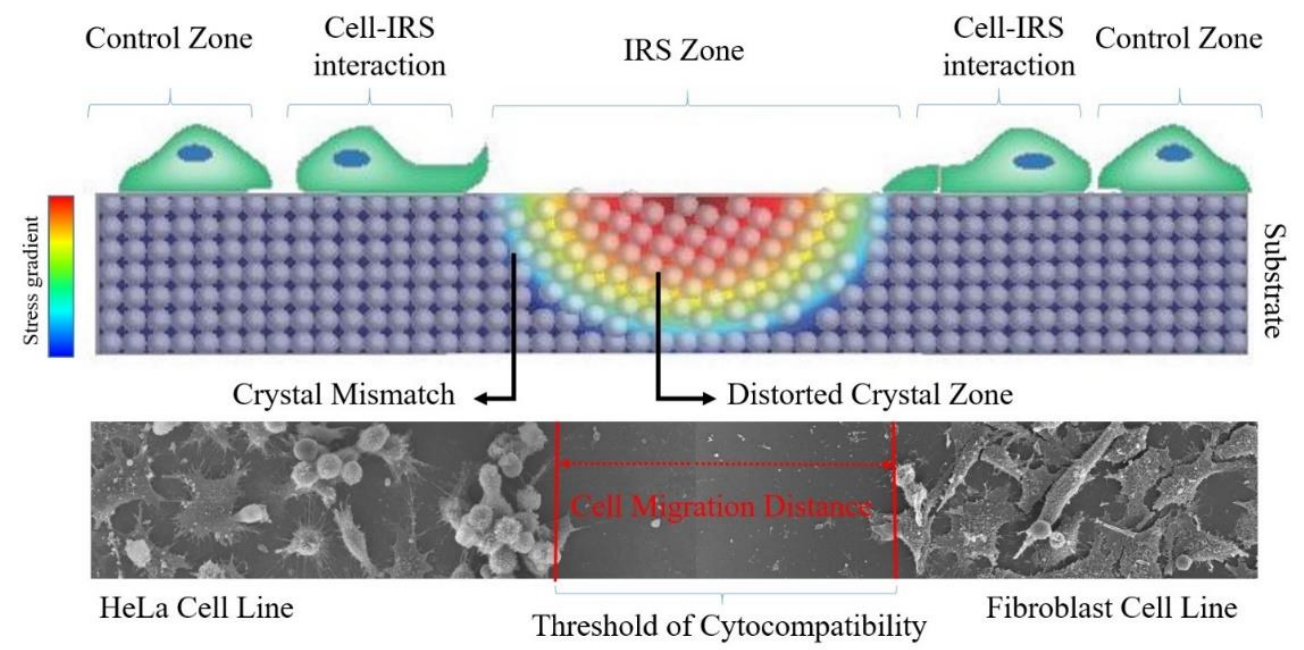

Figure 3.1: Schematic illustration shows the response of cells to IRS. Two types of encounters on the proximity of IRS are: cell breakage and cell protrusion. SEM images of HeLa and Fibroblast (NIH3T3) represent collective cell migration towards stressfree area on the bio-template.

Cells in a real tissue are subject to an environment of three-dimensional space, multiple soluble factors, and cell-cell interactions ${ }^{1,8-9}$. Some effort has been made to partially address this issue. For example, Zhou et al. have demonstrated a two-level topographic pattern made by an integrated lithography to cue cells. Modification of surface chemistry is another approach of bio-template. Premnath et al. have recently performed an in-depth study of programing cancer cells through phase-functionalized silicon-based biomaterials ${ }^{10}$. It revealed that changing the surface chemistry of the silicon substrate by formation of oxides contributes to the control of directional migration, cytoskeleton shape, and cell population ${ }^{10-12}$. This finding has provided valuable insight into 
cellular responses to changes in material-phase stimuli. However, the functionality of this method relies on the changing of surface chemistry. Recent studies have shown that mechanical loading (stress and strain) can be implemented as a cue for cell signaling and migration ${ }^{13-167}$. However, the application of this method is restricted to polymer-based bio-templates ${ }^{17}$. It cannot be applied to silicon-based bio-templates which are widely used as biocompatible templates, due to the brittle nature of silicon ${ }^{18-19}$. In this research work, we introduced a novel concept of using laser induced residual stress (IRS) on a monocrystalline silicon substrate as stimuli for cell modulation. This method does not employ chemicals or topographic structures. It stimulates cell migration and alignment through subsurface stress. To the best of our knowledge, this is the first time that subsurface stress has been used on silicon bio-template for the modification of cytocompatibility. A monocrystalline silicon wafer has been chosen as an experimental substrate because silicon crystals are sensitive to mechanical and thermal excitation. Silicon is increasingly being considered for bio-applications ${ }^{20-22}$. Aside from biological in favor of using silicon as a bio-template, slight changes in the crystal orientation of a silicon substrate leads to the build-up of a residual stress component. This unique property can be attributed to the mono crystallinity of silicon, making it a favorable material for use as a bio-template. In order to accurately tune the functionalization of stress components on the IRS, the bio-template was exposed to the irradiation from an Ultrashort Pulsed Laser (USPL). The combination of shockwave formations and intense thermal expansion generated by the laser irradiation induces crystal distortion. The resulting mismatch of distorted crystal orientation and virgin crystals causes residual stress. The functionality of the stress component was controlled by varying repetition rate (rep.rate) of the laser. The repetition rate determines the level of stress of the functionalized stress component, and it tunes the degree of biocompatibility of the bio-template. This functionalized property elicits precise control on cell behavior, such as directional cell migration and self-cytocompatibility. Fibroblast and cancerous (HeLa) cells seeded respectively onto IRS zones and the responses were observed after 24 hours of incubation. Both cell lines showed a significant collective migration tendency towards virgin areas on the bio-template. In contrast to the cell migration trend, cell mobility was confined by the functionalized stress component. In this case, the migration distance can be regulated by the level of stress within the functionalized stress component. The Fibroblast (NIH3T3) cells are found to be distinctively more sensitive to the functionalized stress component. Precisely speaking, the threshold of cytocompatibility is comparatively lower in Fibroblast (NIH3T3) cells. Moreover, 
directional cell alignment was observed in the proximity of IRS. The results showed that, for the first time, the significant promise of creating self-cytocompatibility properties in silicon biocomponent without resorting to chemical stimuli and/or topographical structures. Figure 3.1 is a schematic drawing shows the response of cells to IRS. The cell interactions, topographical, elemental, compositional, and crystallographic properties of the IRS bio-template were characterized by the Scanning Electron Microscopy (SEM), Florescent Microscope, X-ray Diffraction (XRD), Energy Dispersive X-ray (EDX), and Electron Backscatter Diffraction (EBSD), respectively. Finally, to identify the threshold of cytocompatibility, the minimum level of stress to observe cytocompatibility, was evaluated with Micro-Raman spectrum shift.

\subsection{Materials and methods}

\subsubsection{Materials and laser irradiation}

High-quality polished single crystal N-type silicon wafers with crystallographic orientation of (100) and $625 \mu \mathrm{m} \pm 25 \mu \mathrm{m}$ thickness were supplied by University Wafer. After dicing the wafers

into $2 \mathrm{~cm}^{2}$ square-shape samples, they were ultrasonically cleaned in a 50 degree Celsius acetone bath for 15 minutes, followed by rinsing and drying. In order to induce residual stress, these substrates were exposed to a diode-pumped, Yb-doped femtosecond laser beam (Clark-MXR, Inc.; IMPULSE Series ultrashort pulse laser). This laser system is capable of producing central wavelengths of $1040 \mathrm{~nm}$ and a laser pulse width varying from 214 fs to $1428 \mathrm{fs}$. As well as, the laser pulse repetition rate is tuneable between $4 \mathrm{MHz}$ (Low rep.rate) and $26 \mathrm{MHz}$ (High rep.rate). The maximum operating average power is $16 \mathrm{~W}$. Utilizing an ultrashort pulsed laser (USPL) (femtosecond range) is advantageous since the absorption of multiphoton excitation energy is confined to the localized laser beam spot within the focal point. This results in minimal collateral damage caused by laser-matter interaction. To precisely functionalize the stress component on the IRS, a pulse repetition rate of 4,8,12 and $26 \mathrm{MHz}$, and scanning velocity of $10 \mathrm{~mm} \mathrm{~s}^{-1}$ were implemented. Laser pulse width and average power were set up and remained constant at $214 \mathrm{fs}$ and $16 \mathrm{~W}$ respectively. The laser spot-size at the focal point was adjusted to $10 \mu \mathrm{m}$ diameter after manipulation by a pair of galvanometers through optics lenses. Prior to seeding the cells, the 
sample was irradiated at the focal point by a computerized Galvano scanner to precisely implement and IRS biomimetic architecture.

\subsubsection{Cell culture and seeding}

In order to remove all debris and impurities, samples were ultrasonically cleaned, following the procedure as previously mentioned. Laser treated bio-templates were rinsed with Phosphate Buffered Saline (PBS) (1X strength compose of: $138 \mathrm{mM} \mathrm{NaCl}, 2.7 \mathrm{mM} \mathrm{KCl,} 1.9 \mathrm{mM} \mathrm{NaH} \mathrm{PO}_{4}$, $8.1 \mathrm{mM}$ Na2HPO4) before seeding the cells then dried and sterilized under UV light for 20 minutes. Fibroblasts cell lines (NIH3T3) and human cervical cancer cell lines (HeLa, ATCC, American Type Culture Collection, and ATCC No. CCL-2) were employed in cell experiments to ascertain comparative cell controllability of mammalian and cancer cell lines. Fibroblast (NIH3T3) cells were grown in a DMEM medium containing $10 \%$ heat activated fetal bovine serum with $1 \%$ penicillin-streptomycin antibiotics (Pen-strep). HeLa cells were grown in a DMEM-F12 medium supplemented with $10 \%$ fetal bovine serum and 1\% Pen-strep. Subsequently, the cells were separately cultured on the substrates that are placed in petri dishes with seeding density of 750,000 cells $/ \mathrm{cm}^{2}$ of substrate surface area. The petri dishes were placed in an incubator for 24 hours at 37 ${ }^{\circ} \mathrm{C}$ temperature.

Statistics. All cell assay were carried out at least three times and the data points are averages unless otherwise mentioned. The error bars indicate as mean \pm standard deviations (SD). Statistical significance were completed using one-way analysis of variance (ANOVA), with *p $<0.05$, **p $<0.01$ suggesting significant difference. Additionally, the control samples were compared for each significant level shown in Figures (3.8 and 3.11).

\subsubsection{Cell imaging and morphology}

The surface morphology of the samples, seeded with cells, were observed using a (Hitachi, SU1500) scanning electron microscope (SEM). For this regard, after the prescribed time period, spent medium were aspirated. This was followed by the fixing of samples in $2 \%$ glutaraldehyde in $0.1 \mathrm{M}$ pH 7.3 sodium cacodylate buffer for an hour. Next, the samples were immersed in $0.1 \mathrm{M}$ sodium cacodylate buffer with $0.2 \mathrm{M} \mathrm{pH} 7.3$ sucrose for 20 minutes. Preceded by dehydration as an 
increasing concentrations of alcohol for 20 minutes. The samples were then dried to a critical point. At the conclusion of the experiment, cells were ready to be directly observed using Scanning Electron Microscopy (SEM). Images were acquired at 100x to 10,000x magnification with and accelerating voltage of $10 \mathrm{kV}$, and a probe current of 50 to $60 \mu \mathrm{A}$.

\subsubsection{Fluorescence microscopy}

To conduct fluorescent visualization the samples are first fixed in methanol-free paraformaldehyde followed by incubation in skim milk to prevent non-specific binding. Actin and cytoskeleton are stained by incubation of the samples with phalloidin-Alexa Fluor 488 (Life Technologies) followed by DAPI (4', 6'-diamidino-2-phenylindole, Life Technologies) to stain the nucleus. The samples are studied using an epi-fluorescent Nikon E-400 microscope (Nikon, Canada).

\subsubsection{Electron backscattered diffraction (EBSD)}

A fundamental difference between EBSD and XRD is that X-ray penetrates through the entire thickness of the sample with a $10 \mathrm{~mm}$ beam size, whereas EBSD receives backscattered electrons from the top 10-50 $\mathrm{nm}$ due to limited backscattering depth of the electrons [22]. The local change in the crystal orientation due to laser strike associated with rapid cooling and shock waves can be determined using EBSD. Crystal orientation was measured with EBSD systems, Oxford Instruments INCA interfaced to a Hitachi SU3500 SEM with a LaB6 electron gun. These systems can automatically obtain a crystal orientation map by scanning a rectangular area on the surface of the specimen, which is titled $70^{\circ} \mathrm{C}$ from the horizontal.

\subsubsection{X-ray diffraction (XRD)}

The elemental composition and crystal structure of the substrate was characterized using Rigaku MiniFlex 600 Benchtop X-ray diffraction (XRD) under $\mathrm{Cu}-\mathrm{K} \alpha$ radiation $(\lambda=0.154 \mathrm{~nm})$. Additionally, XRD analysis of the IRS revealed a pronounced (211) peak that emerged when the laser rep.rate was increased. These spectra are in well agreement with EBSD results in Figure 3.5. Secondary phases were not detected in the XRD spectra of IRS samples, which shows there is no compositional deviation from an untreated silicon wafer. To further corroborate with the XRD analysis, Raman and EDX spectra were acquired. 


\subsubsection{Energy - dispersive X-ray spectroscopy (EDX)}

Further surface analytical techniques were employed to identify possible compositional changes on the IRS zone and compression within the untreated silicon surface. The advantage of employing SEM/EDX lies in shallow penetration depth of the interactive electron beam in constract to XRD. Thus, surface elemental analysis can be achieved.

\subsubsection{Micro-Raman spectroscopy $(\mu \mathrm{RS})$}

Micro-Raman spectroscopy is capable of characterizing types of stresses (Tensile / Compressive) within a small measurement spot size, below $5 \mu \mathrm{m}$ in diameter, and a submicron spatial resolution ${ }^{23}$. Although Micro-Raman spectroscopy was mostly being used in chemical composition studies as a complementary technique to other methods, deducing crystallinity information is advantageous to this technique as a non-destructive method to measure residual stress $^{24}$. Raman spectra were obtained using a Bruker SENTERRA, Dispersive Raman Microscope having a central wavelength of $532 \mathrm{~nm}$ as an excitation source. The Raman shift measurements were taken perpendicularly along the IRS of several samples at $10 \mu \mathrm{m}$ intervals. Through the known relationship between the Raman shift and stress, the stress distributions on the lasertreated samples were determined.

\subsection{Results and discussion}

\subsubsection{Fabrication and characterization of IRS onto bio-template}

Interaction of ultra-short pulsed laser with matter falls into two steps: a localized thermal accumulation and shockwave caused by multi-photon absorption, and a blast of plasma formation $^{25-26}$. Thereby the sequential interaction of USPL trains with the substrate is triggered to increase the localized functional stress component along with the laser path ${ }^{27-28}$. Laser irradiation of silicon-based bio-templates using different laser repetition rates has prompted a gradient of residual stress at the peripheral of IRS ${ }^{29-30}$. The amplitude of this laser-induced stressed zone is proportional to the repetition rate of the laser. However for a given pulse width, the gradient of laser fluence decreases with increasing laser pulse repetition rate from $4 \mathrm{MHz}$ to $26 \mathrm{MHz}$; here, 
the transmitted energy, in terms of heat and pressure of the shockwave, is intensified. To expound upon the relationship of laser ablation threshold and laser fluence with repetition rate, the following equations were considered;

Laser fluence is given by,

$$
1
$$

Where $\mathrm{E}$ is laser pulse energy $[\mathrm{J}]$ and $\mathrm{A}_{\text {eff }}$ is effective focal spot area $\left[\mathrm{cm}^{2}\right]$. Laser Pulse energy is given by,

$$
\mathrm{E}=\frac{P_{\text {avg }}}{F_{\text {rep }}}
$$

Where $\mathrm{P}_{\mathrm{avg}}$ is the Laser average power [W] and Laser repetition rate is $\mathrm{F}_{\text {rep }}[\mathrm{KHz}]$. Ablation threshold for a single laser pulse is given by,

$$
T=K \cdot \ln \left(\frac{F_{t}}{F_{t h}}\right)
$$

Where $F_{t}$ is the laser fluence, $F_{t h}$ is the ablation threshold, and $\mathrm{K}$ is related both to the absorption depth of the light in the bulk material, and the thermal diffusion length $(2 \sqrt{ } D \times \tau$ where $D$ is the thermal diffusivity and $\tau$ is the laser pulse duration).
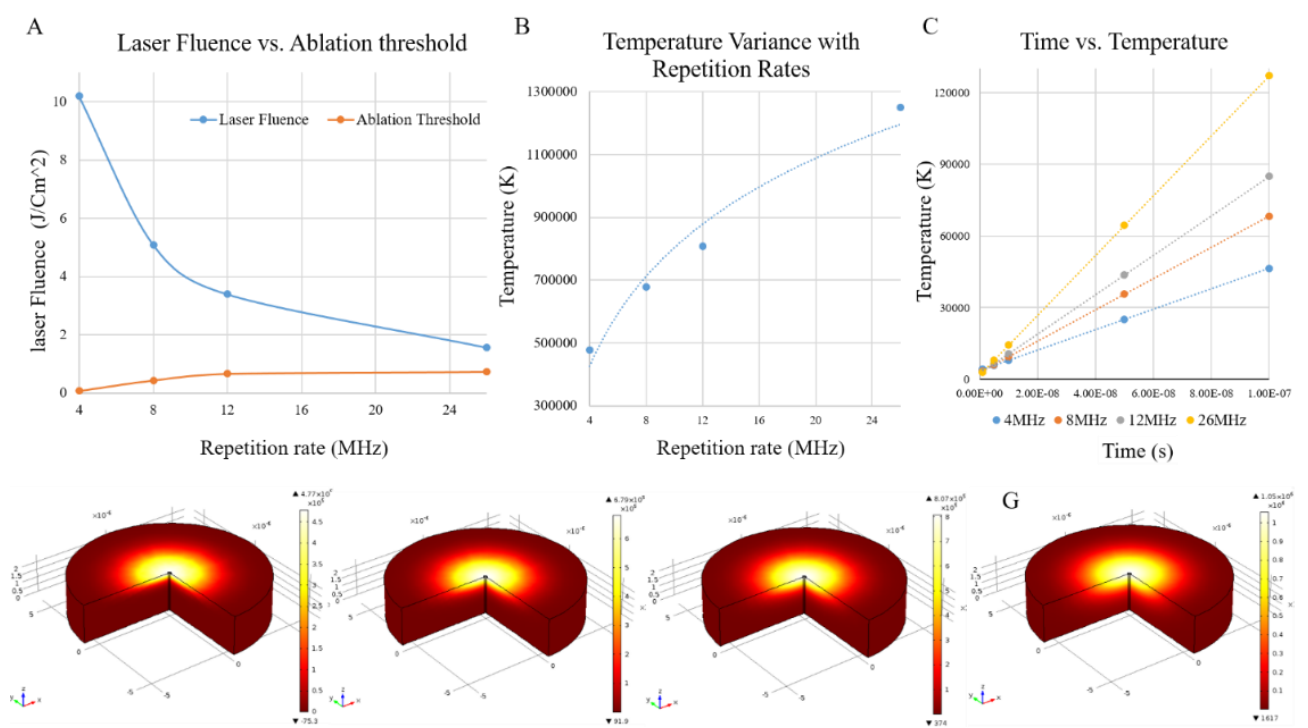

Figure 3.2: Plot A, shows a variation of laser fluence versus ablation threshold of Silicon bio-template a distinct laser pulse width of (214 fs) and repetition rates ranging from 4 to $26 \mathrm{MHz}$. Plot B, Temperature variation with rep.rate, shows temperature rise 
exponentially by increasing laser rep.rate. Plot C, shows the temperature vs. heat dissipation time. Figures D to G show the temperature gradient simulated by COMSOL Multiphysics. (All the data were taken after 10 consecutive pulses).

Figure 3.2 Plot A shows the variation of laser fluence versus the ablation threshold in terms of energy generated per unit area at distinct repetition rates and 214 (fs) laser pulse width. In this plot, the effect of the pulse-to-pulse interval was not taken into consideration. However, multi-photon absorption simulation using COMSOL Multiphysics unveiled that the accumulative influence of laser pulses increases by decreasing pulse-to-pulse interval, that is, laser rep.rate. When the biotemplate is irradiated with a USPL the surface temperature of substrate exponentially rises by increasing laser rep.rate. The difference in temperature between face and subsurface of the silicon bio-template, caused by rapid heat dissipation, creates intricate crystal distortions. This changes the crystal orientation of the IRS zone from inherent (100) to (211) and a different order of crystallinity. Another advantage of utilizing tunable USPL is the ability to precisely modulate the IRS, avoiding collateral damage created by exceeding the ablation threshold of an irradiated biotemplate. In particular, amplifying the repetition rate from $4 \mathrm{MHz}$ to $26 \mathrm{MHz}$ causes extensive accumulation of heat on the exposure zone - owing to maximizing laser-matter interaction time, more thermal and shockwave energy is transferred to the substrate. Therefore, incremental heat generation intensifies thermal stress, resulting in the formation of the laser induced residual stress zone. Earlier mentioned laser parameters such as fluence and rep.rate also have a similar effect in amplifying the laser shockwave.

In an attempt to understand the heat accumulation generated by the interaction between an ultrafast laser and a bio-template, COMSOL Multiphysics was exploited to simulate the average heat flux disturbed during multiphoton absorption. As mentioned, the simulation was created on COMSOL Multiphysics software and the built-in Heat Transfer in Solids Module was used to solve the following heat transfer equation:

$$
\rho C_{p} \frac{\partial T}{\partial t}+\rho C_{p} \boldsymbol{u} \cdot \nabla T=\nabla \cdot(k \nabla T)+Q
$$

Where $\mathrm{k}$ is the thermal conductivity of silicon, $\rho$ is the material density, and $\mathrm{Cp}$ is the heat capacity of the silicon substrate at constant pressure. The pulse width and rep.rate of the USPL were varied for each computational run of the simulation by adjusting the parameters for each new computation. To represent the silicon wafer, a cylindrical geometry was created with a thickness 
of $1 \mu \mathrm{m}$ and with a radius identical to the spot size $(5 \mu \mathrm{m})$ in order to facilitate more precise meshing. A Gaussian pulse (I) was used to mimic the distribution of intensity within a laser beam, the highest intensity being in the center with decreasing intensity towards the circumference. A

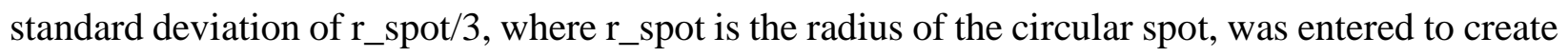
increasingly higher temperatures in the center of the ablation spot. This allowed the simulation of a more realistic temperature distribution. Within the heat flux equations of COMSOL, the laser power (p_laser) was multiplied to the Gaussian functions to produce a gradient intensity (hf) within the laser spot area:

$$
h f(x, y)=p_{\text {laser }} * g p 1(x) * g p 1(y)
$$

The rep.rate and pulse width were incorporated into a single equation to create a rectangular (rect2) pulse train (an3) with parameters that could be manipulated:

$$
\operatorname{an} 3(t, x, y)=\operatorname{rect} 2(t) * h f(x, y)
$$

A heat flux function (an4) was then formulated:

$$
\operatorname{an} 4(t, x, y)=h f(x, y) * \operatorname{an} 3(t)
$$

Finally, within the Heat Transfer in Solids Module, the above equation was entered as a general inward heat flux to produce:

8

$$
q_{o}=\text { emissivity } * \operatorname{an} 4(x, y, t)
$$

Here, emissivity was taken as 0.8. Within COMSOL, the bottom surface of the silicon wafer geometry was thermally insulated and the initial temperature of the wafer was defined at $293.15 \mathrm{~K}$. A free tetrahedral mesh was used on the entirety of the geometry with a maximum element edge length of 1.8E-6 meters. Upon completion of the computations, the wafer thickness was scaled up to five times to allow improved observation of the depth of the heat gradient within the thickness.

For the following study, a classic heat transfer model was applied in order to describe the phenomenon of accumulative temperature rise caused by amplifying the repetition rate from 4 MHz to $26 \mathrm{MHz}$. However, as calculated and plotted on Figure 3.2-A, laser fluence is expected to decrease with increasing repetition rates. Also, shortening pulse to pulse interval at higher repetition rates, increases the initial temperature for the subsequent pulse. Therefore, heat accumulation is expedited by multiplying the number of pulses, as shown on Figure 3.2-B. As seen 
in Figure 3.2-C, heat dissipation time is longer than pulse interval at a higher rep.rate and thus, thermal energy would be expected to build up by increasing the rep.rate. In contrast, at a lower rep.rate, accumulated thermal energy is insufficient, since the heat induced by a single pulse has dissipated by the time the next pulse arrives. Figures 3.2-D to $G$ show the heat generated by multiphoton absorption at different rep.rates, the cross sectional view displays the heat dissipation depth and expansion of IRS at higher rep.rates. The pulse-to-pulse duration is a function of the repetition rate of a laser and therefore, has a significant influence on laser matter interaction - the substrate in the focal point gains energy and is thermally loaded by sequential pulses. Therefore, different repetition rates $(4,8,12$ and $26 \mathrm{MHz})$ at constant a 214 fs pulse-width have been implemented to irradiate the bio-template, through which the functionalized stress component can be induced. Moreover, to characterize the formation of distinct crystal orientations XRD and EBSD have been carried out.
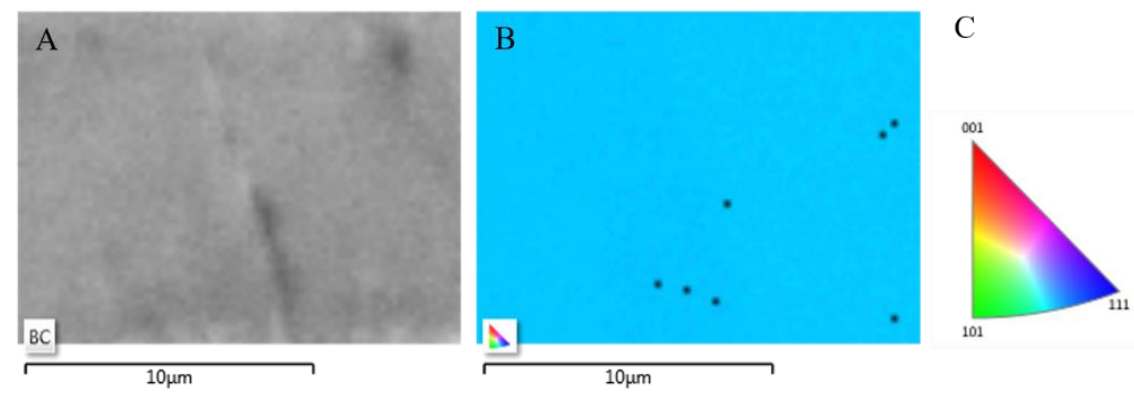

Figure 3.3: A, Backscatter scanning area. B, EBSD mapping of the IRS zone. C, EBSD relative Euler orientation maps. (The color is related to the crystallographic direction that is parallel to the surface)

The crystalline orientation map of the IRS zone is shown in Figure 3.3. The mapping area shown in Figure 3.3 Plot A is $200 \mu \mathrm{m}^{2}$, with a mapping step of $0.2 \mu \mathrm{m}$ where an index of over $85 \%$ was achieved. The different colors of the orientation map correspond to the different crystallographic orientations of scanned area. The (001) crystallographic plane was set as the reference direction. Hence, comparison of collected information from backscattered Kikuchi diffraction patterns at each EBSD mapping point revealed a deviation from the reference orientation. The EBSD mapping result shows that the IRS zone is blue in color, whereas crystal orientation on the unirradiated zone is red in color (according to the EBSD relative Euler orientation map Figure 3.3C) which indicates a deviation from (100) to (211). The color variation of the orientation map shown on Figure 3.3-B corresponds to the different crystallographic orientations. In this case, 
(211) is the dominant crystal plane. The corresponding orientation for each color is represented on Figure 3.3-C. This EBSD outcome is in line with the resulting XRD pattern.

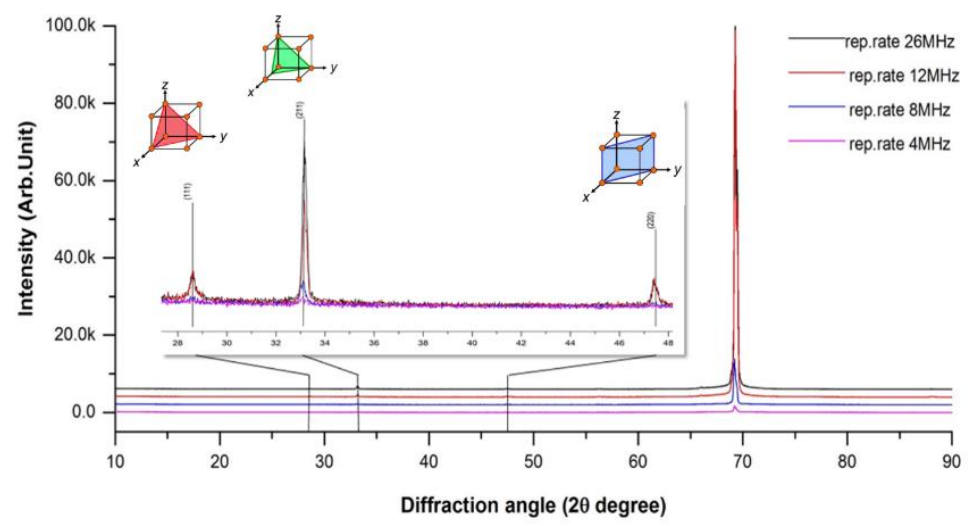

Figure 3.4: XRD patterns of laser irradiated bio-templates at 4, 8, 12, and $26 \mathrm{MHz}$. Enlarged pattern shows emerging peaks, representing of (111),(211), and (221) crystal orientations.

The influence of laser irradiation on the crystal orientation of bio-templates was acquired by utilizing X-ray diffraction, as plotted on Figure 3.4. Diffraction patterns of the IRS bio-templates at different laser repetition rates were compared with a single crystal silicon substrate as a reference. D-spacing calculation of diffraction pattern of reference substrate shows a characteristic peak at $2 \Theta(69.2)$, which stands for (100). While after exposure to an USPL, distinct peaks such as (111), (211) and (220) were emerged. An increment in intensity of a peak represents (211) crystal orientation observed by increasing repetition rate of the laser pulse from 4 to $26 \mathrm{MHz}$. However, this peak has relatively lower intensity compared to a dominant (100) peak due to the fact that only a thin facial layer of a bio-template undergoes crystal orientation changes. The XRD result agrees with the EBSD outcome, both of methods demonstrating changes of crystal orientation on IRS zones. Further characterization has been done using EDX to examine the possibility of surface oxidation or surface compositional changes. The XRD analysis revealed that the compositional aspect of all samples remained intact. Surface compositional characterization of IRS at different laser repetition rates was examined by EDX. Figure 3.5 shows an EDX spectral. Elemental analysis of the spectrum revealed that there is no compositional difference in comparison to a plain silicon wafer. This indicates that multiphoton substrate interaction to induce functional stress component using the previously mentioned parameters, does not cause compositional changes. However, 
alternations in crystal orientation have clearly been observed in XRD patterns and through EBSD mapping.
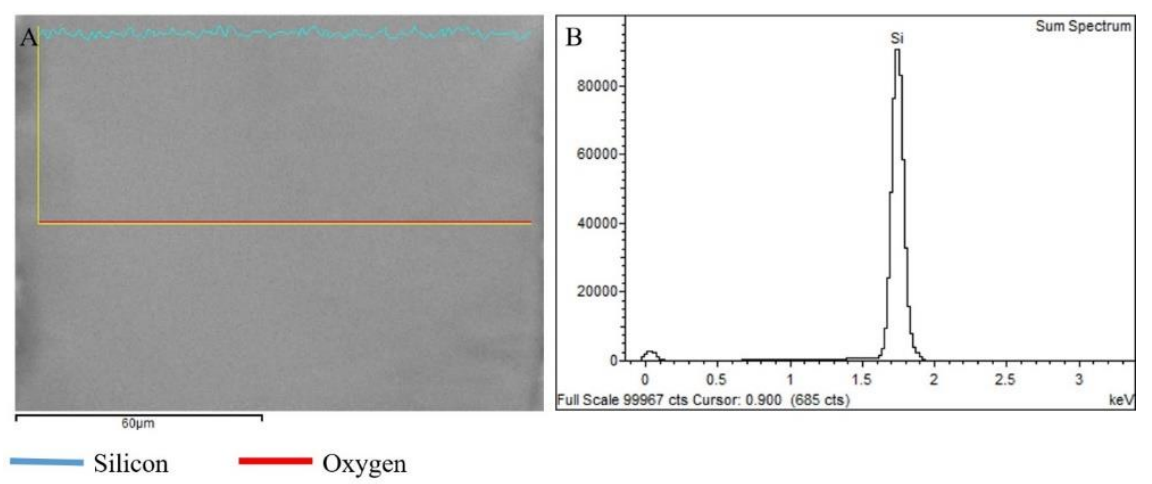

Figure 3.5: EXD spectrum through LIRS IRS and plain bio-template. A, SEM image of take spectrum. B, EDX Spectrum identifying the presence of Oxygen.

To analyze the relationship between laser irradiation energy and functionalized stress components, IRS was characterized by Raman spectroscopy in terms of wavelength shift. In general, as a result of compressive stress, Raman frequency increases while tensile stress results in downward shift of Raman frequency ${ }^{23-24,31-32}$. Figure 3.6-A shows Raman spectra of a bio-template irradiated at 26MHz. As shown in Figure 3.6-A, there is a slight difference in measured peak position when moving outward within the IRS zone. Raman shift variation measured at each $10 \mu \mathrm{m}$ (up to 50 $\mu \mathrm{m}$ ) from the proximity of IRS at different rep.rates, are illustrated in Figure 3.6-B. The characteristic Raman scattering frequency of the silicon-based bio-template used in this study was $\omega=521 \mathrm{~cm}^{-1}$ however, this value shifted as a result of USPL-induced stress. Mapping of Raman shift differences through the IRS zone leads us to determine the thresholds of HeLa and Fibroblast (NIH3T3) cell lines in sensing the minimum required functionalized stress component by which a cell-free zone can be created as a result of collective cell migration. Slight changes in measured frequency, in this case for $0.5 \mathrm{~cm}^{-1}$ peak shift in silicon, represents a stress sensitivity of about 4 $\mathrm{KPa}^{32}$. The relation between Raman peak shift and stress was assumed to be simply linear, and the effect of all stain tensor components are not considered ${ }^{31-32}$. Components of strain in crystalline materials affect the frequency of the Raman phonons and as such, the position of the Raman peak. For instance, for uniaxial or biaxial stress in the (100) plane of silicon, this relation is:

$$
\text { Uniaxial } \sigma(\mathrm{MPa})=-435 \Delta \omega\left(\mathrm{cm}^{-1}\right)
$$


It can be inferred from Figure 3.8-13 that laser irradiation has induced a significant level of residual stress on the bio-template.
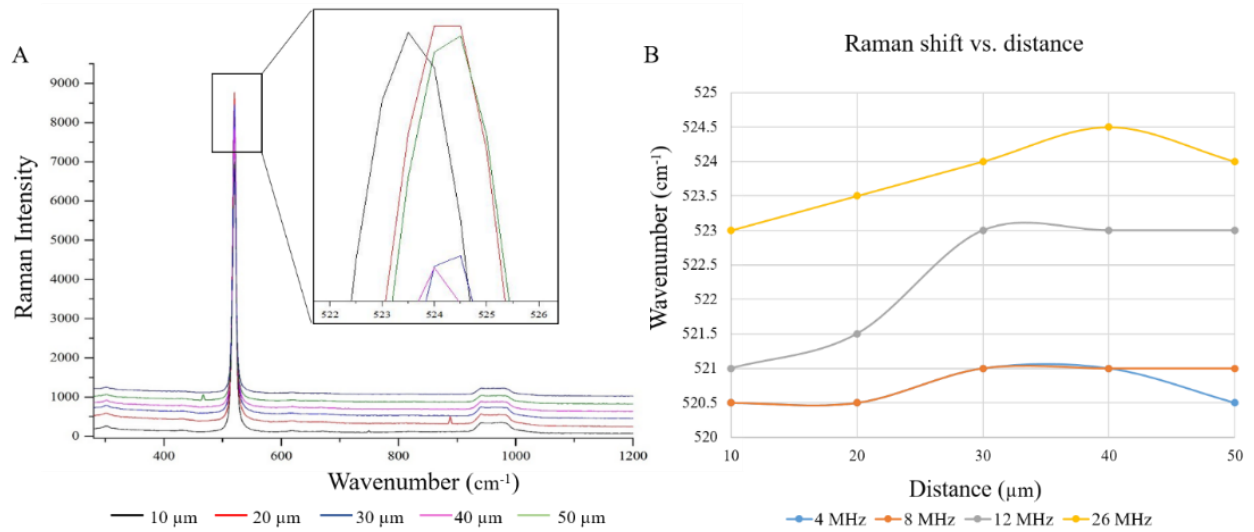

Figure 3.6: A, Raman spectra of IRS at $26 \mathrm{MHz}$. B, Raman shifts at 4,8,12, and $26 \mathrm{MHz}$

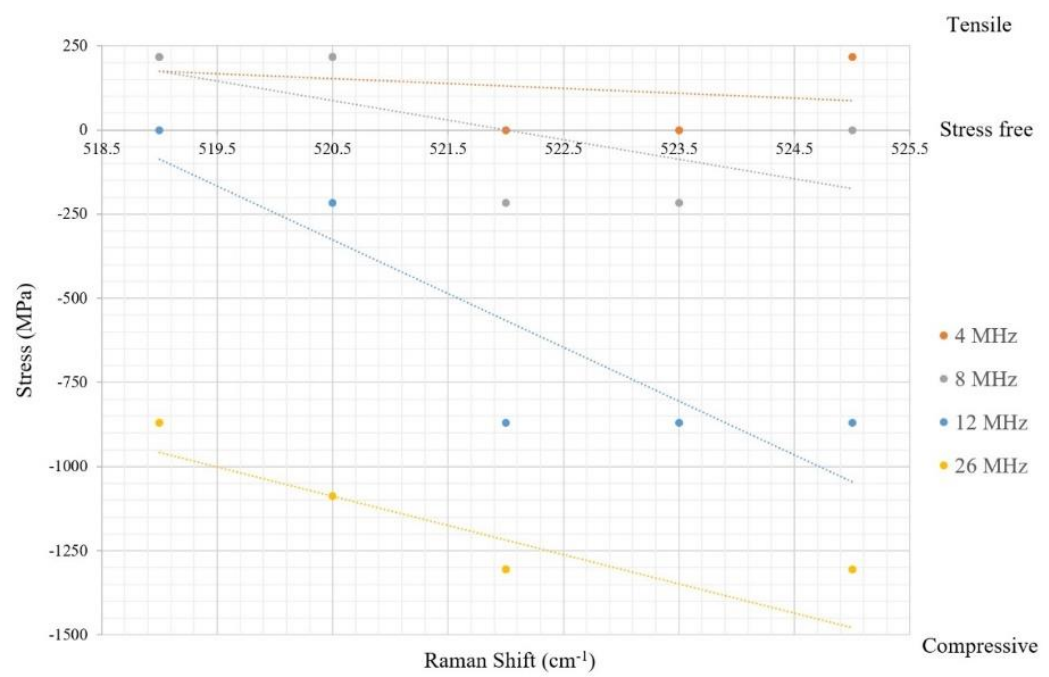

Figure 3.7: Shows magnitude of stress component as function of repetition rate.

Laser fluence that can be precisely tuned through the adjustment of laser parameters, such as rep.rate, laser power, to accurately determine the tensile and compressive stress. The results from cell culture show that the degree of functionalization of the induced stress component depends on the level of stress. A general observation is that a considerable amount of compressive stress was 
induced at rep. rates higher than $8 \mathrm{MHz}$. Comparison of collective cell migration distances from the IRS, demonstrated that compressive stress has a profound impact on cell response.

\subsubsection{Cell interaction with IRS bio-template}

\subsubsection{Collective cell bio-template}

A culture medium that contained $3 \times 10^{6}$ cells $/ \mathrm{mL}$; HeLa and Fibroblast (NIH3T3) cell lines were separately seeded and incubated for $24 \mathrm{hrs}$. Interestingly, collective cell migration from the IRS zone towards the plain surface was observed on fluorescent and SEM images. As shown on images in Figure 3.8 both Fibroblasts (NIH3T3) (Fib) and cancerous cells (Hela) responded to a functionalized stress component as a migration stimulus. Thus the sensitivity of cells to the residual stress required to cue them has been secured as a threshold of cytocompatibility. Moreover, as evidenced in Figure 3.8, higher sensitivity of cells to the compressive stress compared to the tensile stress is significantly noticeable. As presented in Figure 3.8 by increasing the repetition rate, the cells migration distance was extended. It is implied that, as a result of increasing the repetition rate, the intensity of residual stress has magnified in linear regression with the average migration distance of cells from IRS zones. Therefore, distinct boundary on proximity of the IRS zone has created a cell-free zone. Given the cell-free zone indirectly rule out the possibility of topographical changes in terms of surface 20ness, as the profound influence of surface roughness on proliferative behavior of cell by which increment of roughness enhances the cell growth and proliferation is well known ${ }^{33}$. The width of this cell-free zone is directly proportional to the induced residual stress and hence laser repetition rate. It can be seen that induced residual stress on bio-templates can be functionalized in order to regulate cells behavior. 


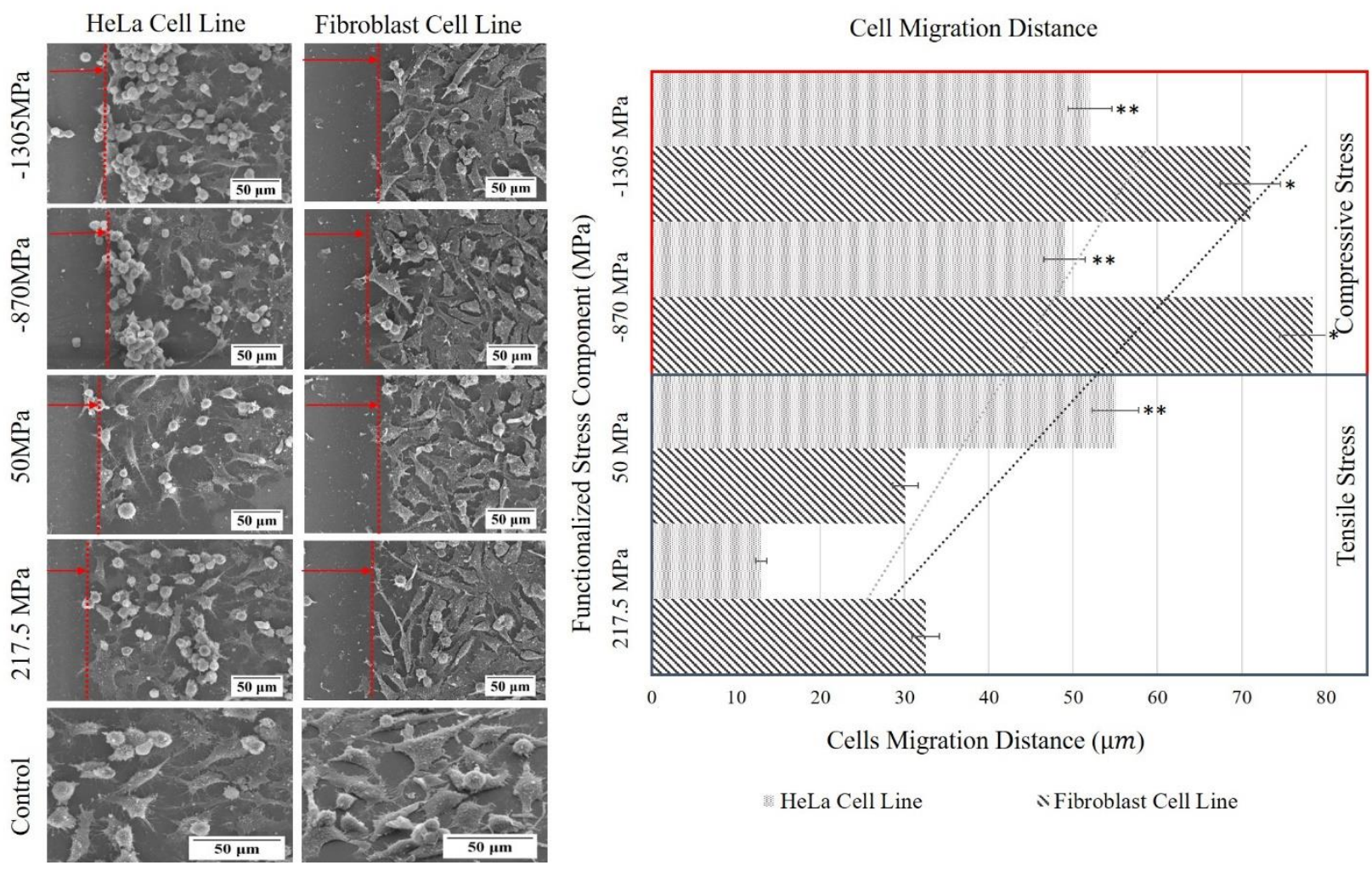

Figure 3.8: Collective cell migration of HeLa and Fibroblast (NIH3T3) in response to functionalized stress component induced on bio-template at different rep.rate are shown on SEM images. The histogram on the right illustrates distance of cell migration corresponding to the magnitude of functionalized stress component. Error bars show SEM; two independent experiments were repeated with $\mathrm{n}=3$ in each experiment. Statistical significance is shown with $*_{\mathrm{p}}<0.05, * * \mathrm{p}<0.01$.

Figure 3.7 shows a significant increase in residual stress at repetition rates greater than $8 \mathrm{MHz}$. The measured Raman shift indicates that compressive stress is dominant at higher laser repetition rates. In addition, transition of tensile to compressive stress at 4 and $8 \mathrm{MHz}$ has been determined to be a threshold of cytocompatibility, by which a minimum width of $30 \mu \mathrm{m}$ for Fibroblast (NIH3T3)and $22.3 \mu \mathrm{m}$ for HeLa have been formed through collective cell migration. Higher sensitivity of Fibroblast (NIH3T3) cells was noticed compare to HeLa cells. Due to the higher mobility and smaller size of the cytoplasm of HeLa cells, their reduced sensitivity to the IRS can be speculated. Additionally, cytoskeleton breakage on cells moving away from the IRS and protrusion on cells who encounters IRS were observed. Cell response to IRS was characterized in terms of cell directionality and persistence distance. The polar diagrams (Figure 3.9) show directionality of collective cells on four different conditions within a $100 \mu \mathrm{m}$ proximity of central 
the IRS zone. Given polar diagrams on Figure 3.9, a significant difference between cell angles on Figure 3.9-A and Figure 3.9-D has been observed, each image compares a tendency of cell alignment on the proximity of the IRS with the control area far from the IRS. To calculate directionality of the cell migration, symmetry of IRS zone was taken into account. The boundary of cell interaction with IRS was defined as $(0,0)$ of the virtual origin, an absolute value of cell angle considered as directional value.

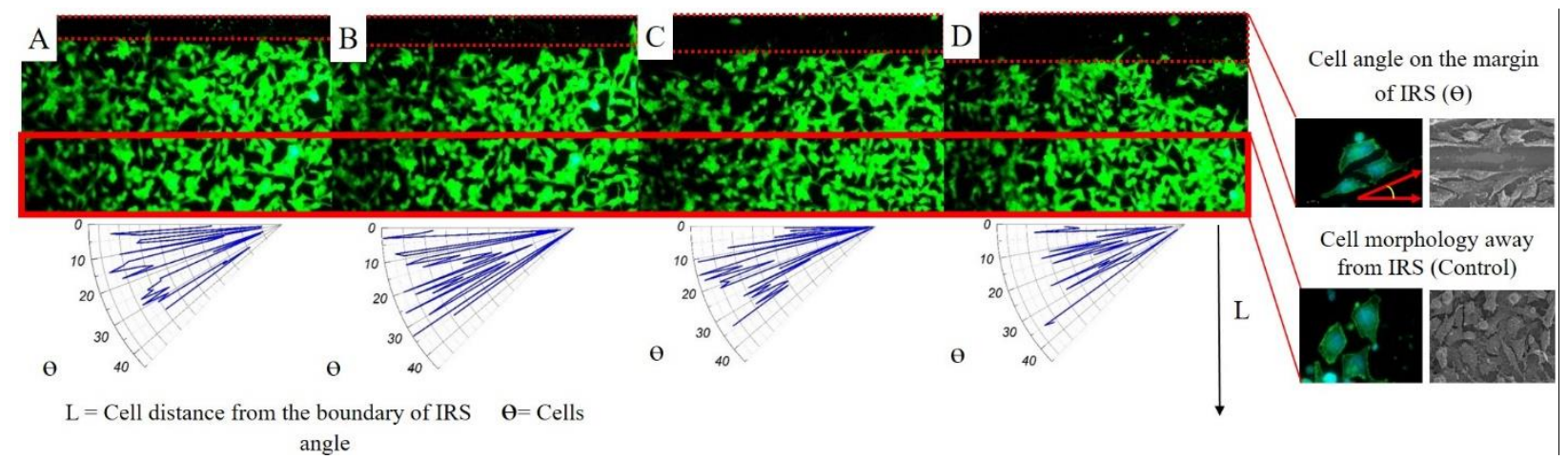

Figure 3.9: Polar diagrams show, decrease of cell angle by increasing the magnitude of functionalized stress component. A-D, The fluorescent images of Fibroblast (NIH3T3) cell lines seeded on the IRS bio-template with different magnitudes of functionalized stress component, 217.5, 50, -870 and -1305 MPa respectively. Comparison of images A and D shows that by increasing the functionalized stress component, diversity of cell angle decreases.

At higher functionalized stress component induced onto the bio-template by shorter laser pulse separation time (high rep.rate), cells tend to be oriented more closely along the $\mathrm{X}$ vector of the virtual coordinate than for a longer pulse separation time (short rep.rate). Cell angles gradually increase towards the unirradiated zone on which cells on this zone do not show preferential direction. In other words, cells tend to become parallel at the proximity of IRS. The same propensity was observed on HeLa cell lines. Preferable migration towards plain areas was pronounced on cells at outer edges of peripheral laser induced zones. This attribute allowed us to manipulate the directionality and morphology of the cells. It can be concluded that cell morphology is an effect of cell migration and self-adoption of a cell's cytoskeleton that implies better understanding of the effects of controlling cell behaviour. Although the morphology of cells away from the IRS zone (control area) showed a tendency toward a polygonal shape. 
Fibroblast Cells

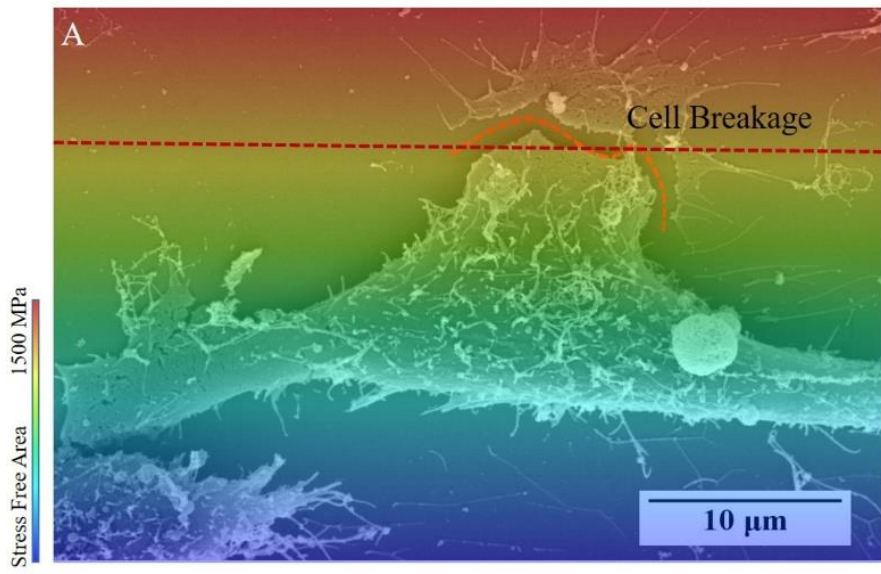

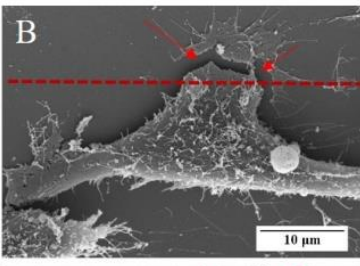

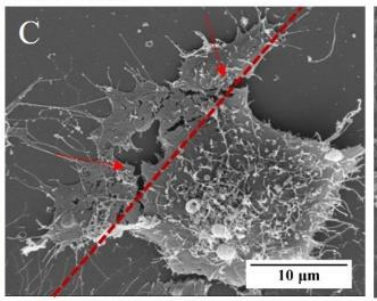

Hela Cells

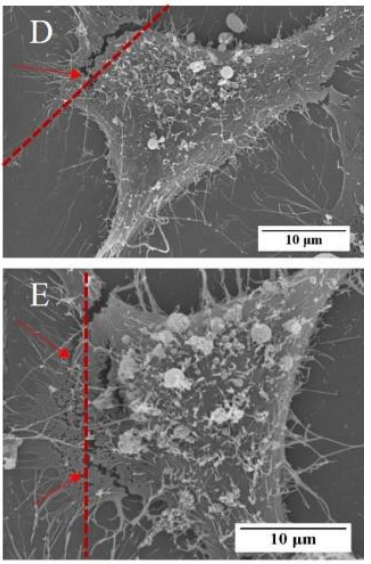

Figure 3.10: A, Schematic illustration of the distribution of functionalized stress component and cyto-breakage as a result of cellIRS interaction. B-C, SEM images shows cyto-breakage of Fibroblast (NIH3T3) cells. D-E same phenomena has observed on HeLa cells facing IRS as well. (Arrows and dashed line indicate the side of breakage and the boundary of IRS zone respectively)
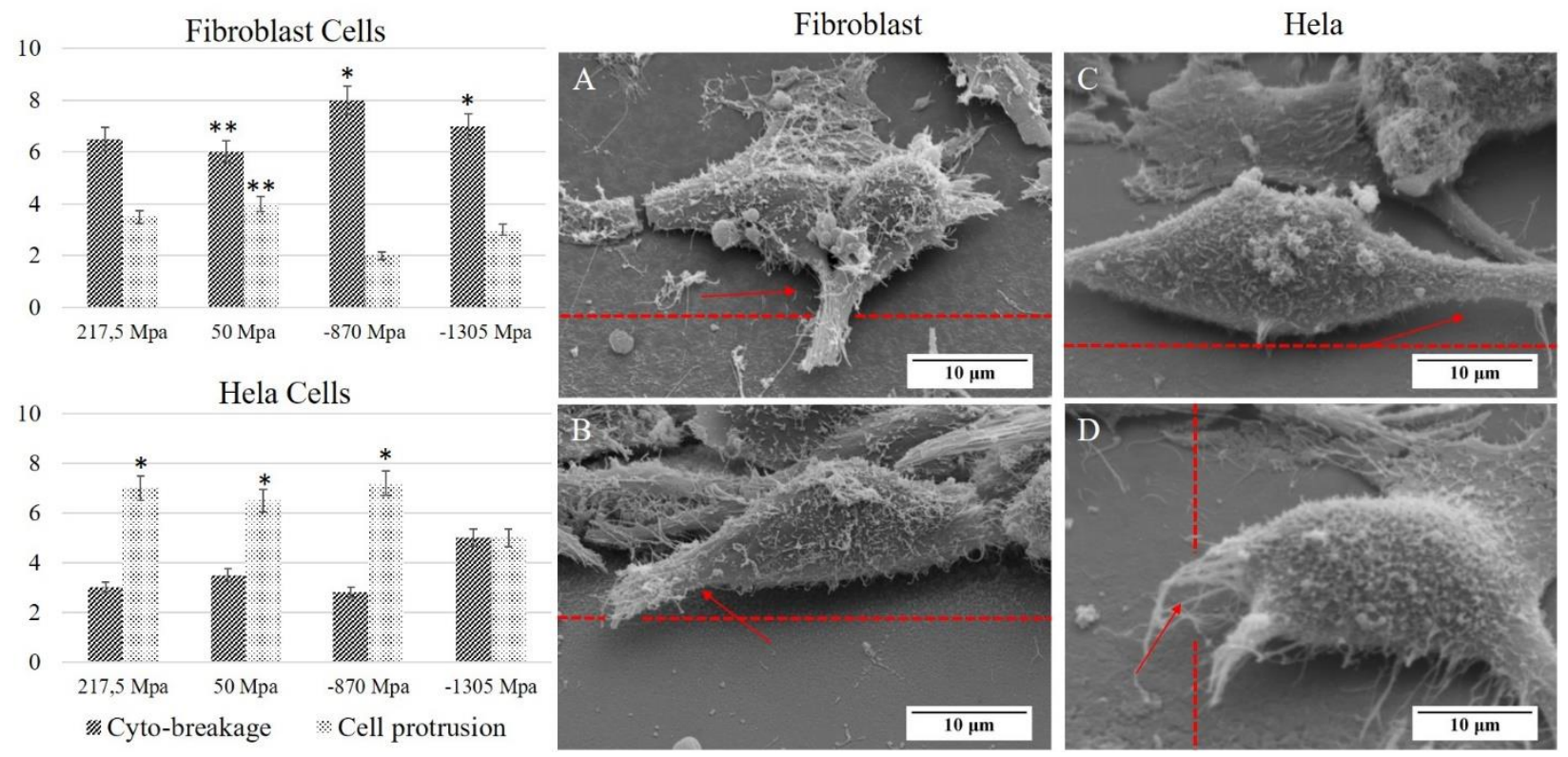

Figure 3.11: SEM images show cells protrusion on the side facing IRS. A-B Fibroblast (NIH3T3) cell line, and C-D Hela cell lines. The statistical diagram compares the number of cell protrusions and cyto-breakages on 4,8,12 and $26 \mathrm{MHz}$ for Fibroblast (NIH3T3) and HeLa. Error bars show SEM; two independent experiments were repeated with $\mathrm{n}=3$ in each experiment. Statistical significance is shown with $* \mathrm{p}<0.05, * * \mathrm{p}<0.01$. (Arrows are indication the lamellipodium protrusion on the SEM images and dashed line indicate the boundary of the IRS zone) 


\subsubsection{Cytocompatibility to induced residual stress (inter-cellular response)}

Quantitative information about cells-matter interaction can be inferred by investigation of cell morphology ${ }^{34-35}$. Therefore, interaction of an individual cell with functionalized stress component was examined. Two distinct phenomena of cyto-breakage and cell protrusion were observed on cells in the proximity of the IRS zone. Upon formation of filopodia after seeding the cells on the bio-template, they perpetually sense their environs to further formation of lamellipodia and ultimately direct cell movement. Cell retraction by which cyto-breakage occurred was reaction to failed adhesion and premature formation of filopodia facing the IRS. The SEM images of HeLa and Fibroblast (NIH3T3) cells, shown in Figure 3.10, indicate that although filopodia have been developed on the both sides of cells, cyro-breakages have taken place on the side of the cell facing to the IRS zone. This is evidence that cell migration through retraction and expansion is prohibited by a functional stress component. On the other hand, during 24 hours of incubation, it is likely that cells migrate towards IRS zone. In this case, cells tend to protrude on the side facing the IRS zone as shown in Figure 3.11. Additionally, qualitative observation exhibited that cell protrusion is more dominant on HeLa cell line compared to Fibroblast (NIH3T3) cell line, in which cyto-breakages was common. Lamellipodia are the actual motor which pulls the cell forward during the process of cell migration. Protruding of lamellipodium, indicates no preference of a cell to attach to the IRS zone as SEM images showed on Figure 3.11 A-D. Therefore, disinterest of lamellipodia to the IRS zone causes cell protrusions. On the other hand, there is a strong belief that the cell crawling in the process of migration is initiated by the development of stable filopodium-substrate adhesion, followed by development of lamellipodium ${ }^{36-38}$. Hence cell protrusion is dependent on collaborative interaction of filopodia and lamellipodia ${ }^{39}$. However lamellipodia function as key structure on cell migration, filopodia extensions are essential for guidance and cellular architecture $^{40}$. Investigation of cell protrusion were made possible by high resolution SEM imaging and tilting technique of substrate up to $45^{\circ}$. 

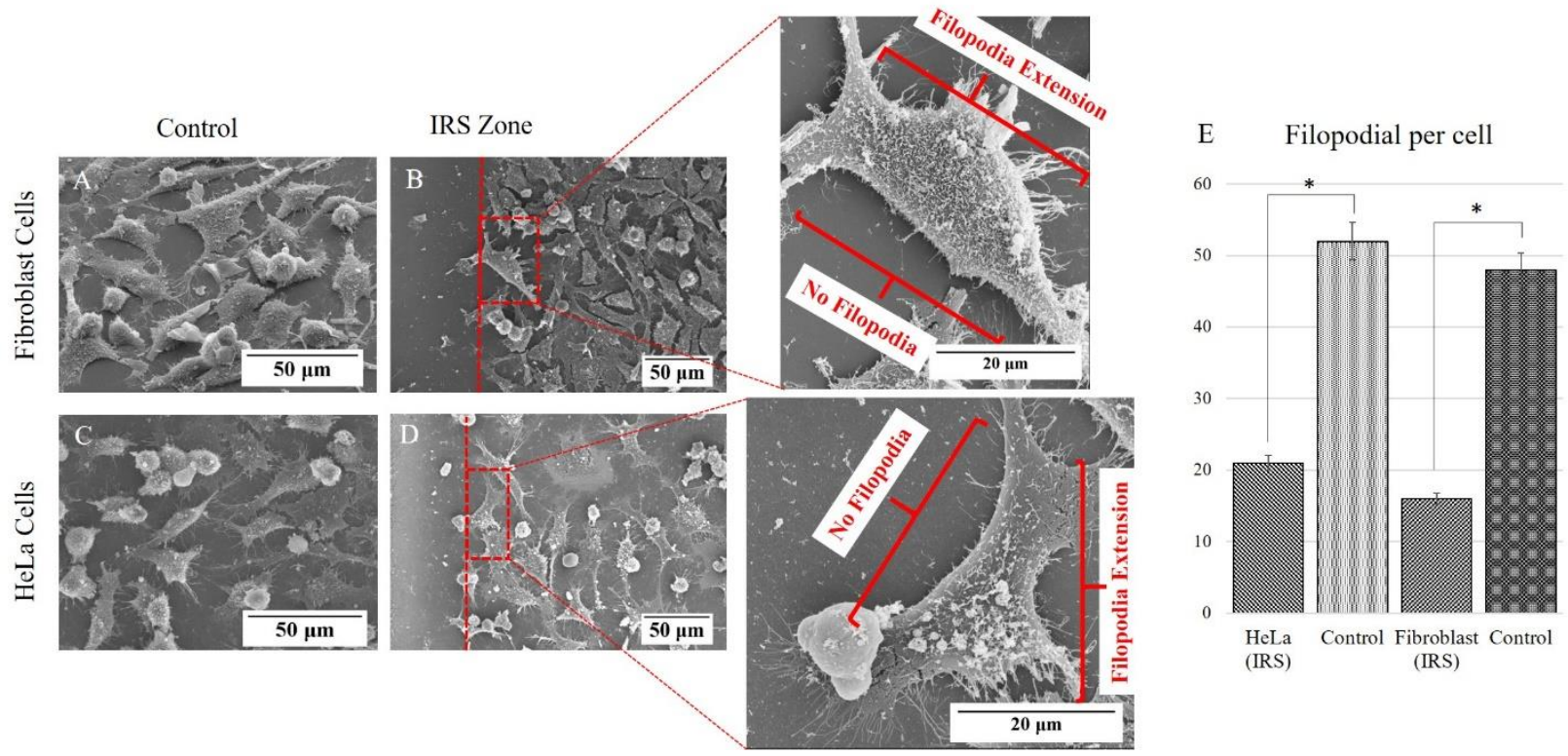

Figure 3.12: $B$ and D SEM images show the Filopodial extension on the side of the cells opposite to the IRS zone. A and C illustrate the Fibroblast (NIH3T3) cells and HeLa cells respectively on the control samples. Bar graph on the left side (E) comparing the number of filopodia of HeLa and Fibroblat seeded on the IRS bio-template and respective control samples. (Hundred cells were counted for each cell group of samples; error bars denote SD. for each group)

\subsubsection{Filopodial extensions}

Filopodia serve as probing sensors at the frontier of cells that are extended beyond the leading edge of lamellipodia. This attribution leads them to have a significant function in cell migration as well as cell environmental sensing ${ }^{37}$. The number of filopodial extensions are the evidence of cell preference to adhere and grow ${ }^{41}$. Quantitative image analysis, revealed that the side of cells facing the peripheral of the IRS zone has relatively no or a reduced number of filopodial extensions compared to the opposite side of cell that faces untreated substrate (control area) as depicted in Figure 3.12 E. This immature development of filopodial extensions was observed on both cell lines of HeLa and Filapodia as shown on Figure 3.12. In addition, the formation of new filopodia has proven to be strongly dependant on pre-existing filopodia. Taking into account the attributes of filopodial, the two main functions of environmental sensing and driving force generated for cell migration together with filopodial-originated actin bundles, best describe the role of these needlelike structures in directing cell migration ${ }^{42-43}$. Filopodia are the starting point for essential adhesion 
and movement. Therefore, the final cellular position, actin bundles, and formation of new filopodia are affected by interaction of these structures with IRS.

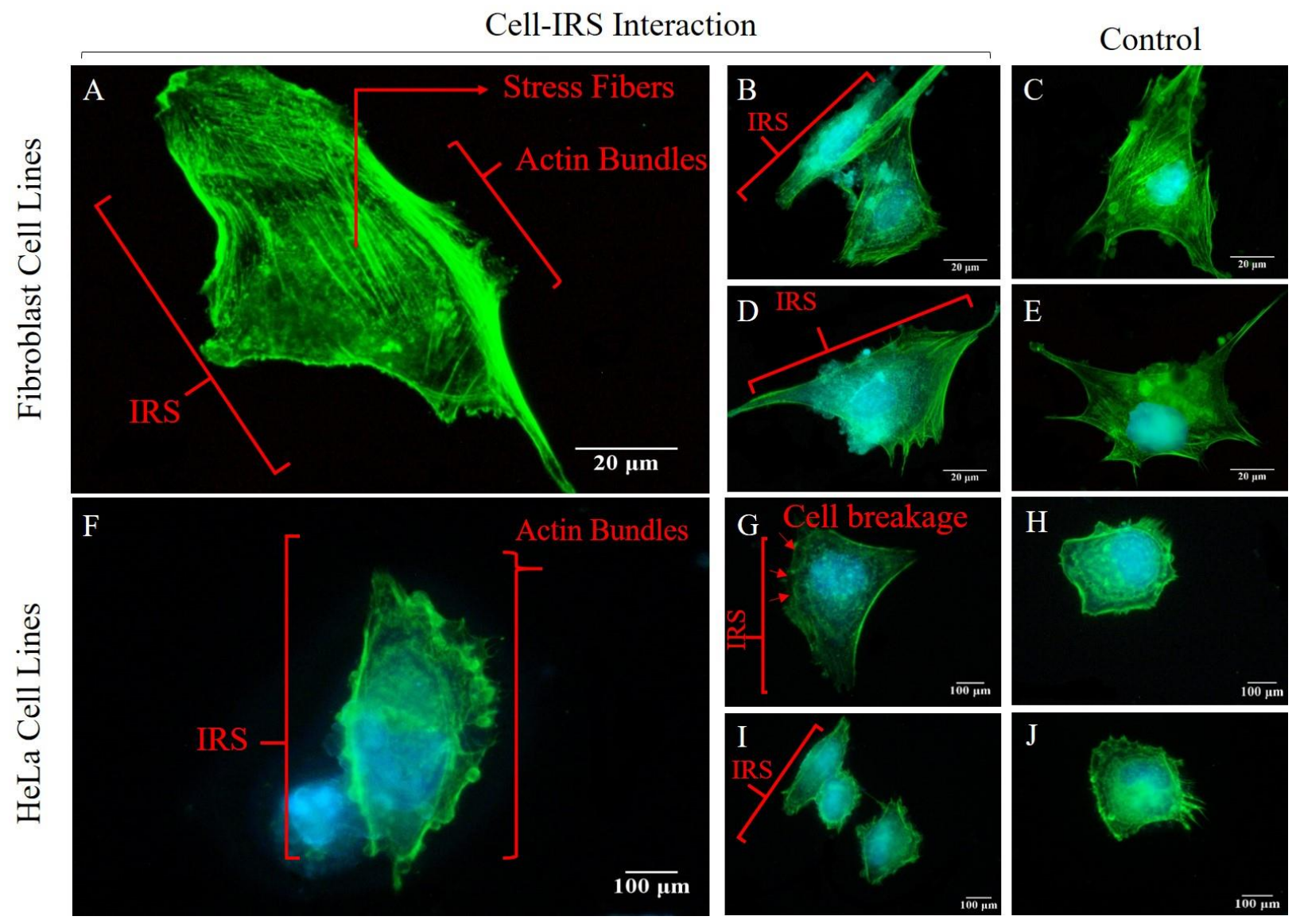

Figure 3.13: Fluorescent images of single cell analysis of Fibroblast (NIH3T3) and HeLa cell lines. A and F show single Fibroblast (NIH3T3) and HeLa cell interacting with IRS, arrows indicate both sides of a cell. B and D are example of Fibroblast (NIH3T3) cells seeded on the laser irradiated bio-template at 26 and $4 \mathrm{MHz}$, compared to $\mathrm{C}$ and $\mathrm{E}$ where extension of filopodia and lamellipodia are directional. G and I show the HeLa cells seeded on the laser irradiated bio-template at 4 and $26 \mathrm{MHz}$ respectively (arrows indicate the cell breakage on side of cell facing the IRS). $\mathrm{H}$ and $\mathrm{J}$ are HeLa cells on the control area. (Optical images showing actin cytoskeleton (green) and nuclei (blue) of cells after 24 hours of culture on IRS bio-template)

\subsubsection{Stress fibres and cell migration}

Stress fibers play an important role in providing numerous functions such as cellular contractility, cell adhesion, and migration ${ }^{44}$. Upon attachment of a cell to the bio-template, the pulling force required for traction is provided by adhesion. Thus, cell transition processes are made possible by contraction of the cell body to the new site. Vacillant cells such as Fibroblast (NIH3T3) showed 
oriented ventral stress fibers perpendicular to the vector of migration. Moreover, a higher concentration of actin bundles formed on the locomotion face of cells are observed. This is caused by stronger adhesion at the migration side compared the rear. Cells in the proximity of IRS have shown, actin bundle concentration on the opposite side of cell breakage, while stress fibers are stretched perpendicular to the direction of migration. (Figure 3.13). Taken together, here we reported a novel method of remote stimulation of cell migration by laser-induced residual stress on silicon-based bio-template. It provided a precise manipulation of cell-bio-template interaction, unlike topographical and/or chemical surface modification, cell response to IRS relies on environmental probing of cells.

\subsection{Summary}

In this commentary I conclude a highly coordinated cell behaviour based on cell interaction with IRS on a bio-template which regulates filopodia formation, adhesion, and collective cell migration. An USPL was employed to modulate the functionalized stress component of IRS. It was found that varying repetition rates have a significant influence on the magnitude of functionalized stress component through which distance of cell migration can be controlled. Cell behavior indicates that there is a necessity of a minimum induced residual stress onto the bio-template in order for a cell to respond, which has been defined as a threshold of cytocompatibility. This was confirmed using fluorescent and SEM imaging to identify the cell morphology and direction at the periphery of IRS. Taking into account the function of filopodia on environmental sensing and direction of migration, we can verify the importance of the filopodia to perceive the presence of residual stress. Moreover, accuracy of the USPL for precisely modulating the functionalized residual stress proves to be an advantage in manufacturing a mimetic architectural lab-on-a-chip. With this, various biomedical applications such as drug testing, fundamental biological studies, and toxicology studies can be carried out. The findings of this study opens a new front on cell regulation and cell patterning by means of virtual stimuli which has shown to be perceivable by cell's environmental sensing. 


\section{CHAPTER 4}

\section{POLYMORPHIC SELF-ASSEMBLED SILICA NANO-WEBS AS A HELA- CENTRIC BIO-TEMPLATE TOWARD AN INTENSIFIED APOPTOSIS PATHWAY}

Keshavarz, M.; Tan, B.; Venkatakrishnan, K. "Cell Selective Apoptosis Induced by Polymorphic Alteration of Self-Assembled Silica Nanowebs." ACS Applied Materials \& Interfaces 2017, 9, 6292-6305.

\subsection{Abstract}

The biocompatibility of silicon-based nanomaterial makes it suitable for biophysical and biomedical applications. However, the application of silicon-based nanomaterials has been mainly restricted to nanoparticles (NPs) as a potential drug carrier and the extracellular matrix (ECM) as a platform for cell adhesion and proliferation. Here, we introduced silica NPs self-assembled into a 3D nano-web architecture that was shown to inherit the therapeutic and proliferative attributes of both NPs and ECMs. The self-assembled silica nano-web (SNW) has, therefore, not only shown targeted drug-like behavior in HeLa cells without the use of bio-markers but also has shown ECM characteristics. The ECM characteristics of SNWs enhanced the cellular attraction and proliferation by which fibroblasts exhibit tissue-like behavior, and HeLa cells underwent an intensified induced apoptosis. These properties are tailored by the alteration of the polymorphic heterogeneities of the SNW as a novel nano-biointerface for exceptional apoptosis induction through the enhancement of cellular attraction, which, to the best of our knowledge, has not been previously reported. These attributes enable selective functionality with which cancerous HeLa and mammalian fibroblast cells were affected differently. Moreover, simultaneous control of the packing index and crystallinity of the SNWs, to which the cells had been attracted, possessed the additional advantage of modulating the selective functionality of this nano-biointerface. These polymorphic characteristics were tailored by the alteration of the crystallinity of the synthesized SNW via precision control of the ionization level of the silicon substrate, whose requisite ionization energy was generated by an ultrashort pulsed laser. Our results showed that the 
therapeutic functionality of the SNW-plated template can be elucidated via the endocytosis of amorphous SNWs. Due to the efficient cellular attraction and remarkable contrast in the cellular response to the SNW-plated template, we expect that these findings will provide new insights and opportunities for designing and engineering novel cell-material interfaces for advanced biomedical applications in cancer research.

\subsection{Introduction}

The emergence of nanotechnology in the last few decades has revolutionized the forefront of cancer therapy. Furthermore, the use of silicon-based nanomaterials in biomedical applications has been growing due to their non-toxicity, stiffness, biodegradability, and biocompatibility. Thus far, silicon-based nanomaterials have been primarily implemented either in the form of nanoparticles or nanostructures. Silicon nanoparticles (SNPs) have been predominantly employed as a carrier for drug delivery or as a core material coated with biomarkers ${ }^{1-6}$. Applications of SNPs as a drug carrier in the field of cancer treatment has been promising for targeted therapy, however, as synthesized SNPs require post-processing such as chemical surface modification for targeting specific cells. Therefore, the conjugation of proteins or specific biomolecules with nanoparticles as biomarkers is an essential step towards therapeutic and diagnostic applications ${ }^{7-11}$. Porous silica nanoparticles, for instance, have shown considerable attention as potential drug carriers because of their high stability and biocompatibility ${ }^{12-18}$. Mesoporous silica nanoparticles synthesized by Meng et al. via the sol-gel method exhibited cancer cell killing with aid of doxorubicin and Pglycoprotein siRNA labeling ${ }^{19}$. Similarly, Rosenholm et al. developed a hybrid mesoporous silica nanoparticle tagged by poly (ethylene imine) and different target moieties ${ }^{20}$. Mesoporous silica nanoparticles synthesized by Zhang et al. exhibited an additional distinction by which selective cancer cell therapy can be achieved by the addition of folate-targeting agents ${ }^{21}$. Thus, additional drugs such as doxorubicin are required to be conjugated with carriers for therapeutic purposes. While SNPs used as logistic carriers are biocompatible, the risk of cross-contamination of cells/molecules through chemical surface modification exists. Moreover, the interaction of chemical composition (used as a post-processing step for surface modification of SNPs) with cells may result in an unexpected phenotype ${ }^{22}$. In sum, it is still an imposing challenge to take advantage of SNPs without the use of a chemical modifier ${ }^{23-25}$. Additionally, to the best of our knowledge, 
the polymorphic heterogeneities of structured silica without the use of bio-labels have not been studied.

Furthermore, recent studies have demonstrated that the employment of nanostructures can provoke the proliferative behavior of cells. Nevertheless, the employment of nanostructures has been exclusively limited to the extracellular matrix (ECM) as a template by which cellular behavior can be modulated in a particular manner, such as controlling the fate of the cell to adhere and proliferate ${ }^{26-27}$. Topographical changes of cell-biomaterial interface have also led to advancements in cell patterning, cell isolation, and collective cell migration ${ }^{28-29}$. The ECM, therefore, has been implemented to control the size and shape of cells as well as the enhancement of proliferation for tissue engineering and wound repair. In addition, micro/nano-structures such as nanofibers have been applied for cell culture and scaffolds ${ }^{30-31}$. These extensive studies improve the understanding of the fundamental behavior of cells and cell-material/cell-microenvironment interactions and promote the development of tissue-engineering materials for advanced biomedical implants and devices. Santos et al. for instance showed the importance of physical and chemical properties of the nanostructured platform on cell growth and proliferation. Moreover, cellular behavior was investigated and it revealed that cell adhesion, viability and morphological changes of in this case Hela and fibroblast cell lines are dependent on the level of protein adsorption on a modified surface $^{32}$. Hence, an extended surface area of the nanostructured platforms is the favorable fate for the cells to adhere and proliferate as adsorption of proteins drastically increases by expansions of surface area $^{33-34}$. Along with the development of micro/nano-fabrication, silica nanostructures have been generated. Through these silica nanostructures, engineering of cellular behavior can be achieved via the design of functionalized three-dimensional (3D) nanostructures with spatiotemporal chemical/physical attributes. Indeed, 3D nanostructures are an important part of the cell microenvironments, such as the nanoscaled villi and podia of cell surfaces and the nanofibers of ECM. Hence, the chemical/physical aspect of nano-biointerface interactions has progressively increased as an essential tool for biological studies and applications ${ }^{34}$. Moreover, important biological studies in recent years, such as cancer diagnosis and therapeutics, have raised the requirements for precise nanoscaled control of cell-material interactions ${ }^{35}$. Together, these factors encourage the creation of more advanced and smarter biointerfaces at the 3D nanoscale. Thus far, extensive studies underline the biocompatible capabilities of silica nanostructure temples for tissue engineering, direction and cell growth control ${ }^{34-35}$. However, the biofunctionality of 
silica-based nanostructures on which self-attractive characteristics and enhanced cell-induced apoptosis are based, as well as their efficacy for different cell lines, has not been reported.

Despite the exploitation of silicon-based nanomaterials in biomedical applications, to the best of our knowledge, the therapeutic properties of SNPs and cell-capturing attributes of silica nanostructure have not been concurrently studied. Furthermore, not only has the cellular interaction with 3D structure, in this case the silica nano-web, not been fully investigated, but more importantly, their interaction with mammalian and cancer cell lines with respect to their polymorphology remains unknown. Unlike conventional nanostructured biointerfaces, selfassembled silica nano-webs (SNWs) have a series of unique attributes provided by multi-photon ionization, with which crystalline or amorphous silica can be formed. Here, we report a HeLacentric nano-biointerface tailored by laser ionization to be efficiently attracted to both cancer and mammalian cell lines followed by selective functionality. While this method is superior for the induction of apoptosis pathways in HeLa cells, fibroblast cells exhibited a tissue-like structure. Hence, the SNW functionalized from the dormant state of silicon was converted to a drug-like therapeutic for HeLa cells, whereas it showed attraction and enhancement of proliferation for fibroblasts inherited from the ECM.

We investigated the effects of an amorphous (Amo) and crystalline (Cry) structure of SNW on HeLa and fibroblast cellular behavior. To the best of our knowledge, neither the effect of the polymorphic property of silica nanostructure on cellular response nor the interaction of a nanoweb framework with cells has been investigated. The findings show that both HeLa and fibroblast cell lines were attracted to the bio-template plated with the SNW. Strikingly, both cell lines exhibited a distinct response to the crystallinity changes in which a close interaction of fibroblasts with amorphous and crystalline SNW was observed. HeLa cells, however, interacted with amorphous silica through an explicit apoptotic pathway. The selective functionality of this material was examined after 24 and 48 hours of incubation for each cell line. Fibroblast cells exhibited a well-stretched cytoskeleton. In contrast, HeLa cells exhibited premature filopodial extension, a decreased number of filopodia, cell clustering, and a small round shape indicating an apoptotic pathway $^{31,36}$. Moreover, the precise control of the ionization level during synthesis enabled us to tune the crystallinity and yield of the SNW, which permitted the simultaneous study of the effects of varying packing indexes and amorphization of the SNW on the cellular response. The results showed that not only did increasing the packing index of the SNW escalate the attraction of 
fibroblast and HeLa cells, but it also enhanced the proliferative behavior of fibroblasts, whereas in HeLa cells, the efficiency of the induced apoptotic pathway increased.

Here, we report the induced apoptosis-like mechanism of silica amorphization on cancer cells, particularly the HeLa cell line, through the endocytosis-induced apoptotic pathway using multiphoton-matter ionization as a versatile synthetic method. Scanning electron microscopy (SEM) showed that the overall membrane structure of HeLa cells remained intact after being attracted by Cry-SNW; however, the cytoskeletons became rounded. In contrast, the number of apoptosis-like cells with porous cytoskeletons increased on the Amo-SNW in both the plated and free-standing form. It is well known that nanomaterials are capable of entering living cells, often by utilizing cellular endocytic mechanisms ${ }^{37-39}$. Therefore, we characterized and examined the cellular internalization/endocytosis (endocytosis, in which nanoparticles are engulfed by the cell membrane and drawn into the cell, leading to a loss of cell wall integrity), viability and cytotoxicity for this SNW. The penetration-induced cell morphological changes to the cytoskeleton were explored by SEM observation. In addition to this imaging technique, an elemental analysis using energy-dispersive X-ray spectroscopy (EDX) revealed a remarkable presence of SNPs within the cells seeded on the template dispersed with the Amo-SNW. High-resolution transmission electron microscopy (HR-TEM) provided evidence of amorphization under higher ionization conditions. The electron diffraction pattern and Raman spectroscopy further revealed that by precisely tuning the ionization energy, an SNW composed of either the crystalline and amorphous structure with different SNC size distribution or both can be synthesized. In vitro experiments showed that the efficiency of SNWs in attracting cells is inversely correlated with the size of the SNCs. Moreover, SEM and Fluorescence Microscopy (FM) analysis revealed a promising ability of the SNW-plated template to attract both cell lines, while the intact proliferative behavior of fibroblasts exhibited the non-toxicity of WNSs after 24 and 48 hours of incubation. Literatures surrounding the cytotoxicity of crystalline and amorphous silica are mainly limited to the consequences of inhalation of SNPs on lung fibrosis ${ }^{40}$. However, there are few studies indicating that uptake of amorphous SNPs induces apoptosis pathway to HeLa cells whereas fibroblast cells are not affected by either crystallinity of silica ${ }^{41}$. Further observation revealed that the adherence of HeLa to the deposited WNS could increase the permeability efficiency of cell membranes. Quantitative and qualitative studies on the adhesion, viability and morphology of the cells were conducted using SEM analysis and FM. Additionally, the physicochemistry properties of the experimental samples 
were evaluated using EDX and Raman spectroscopy. Taken together, the findings suggest that the induced apoptosis-liked mechanism of SNWs is likely as follows: the SNW attracts cells by providing a preferred area of adhesion, and then internalization causes cell membranes to rupture, resulting in the final apoptosis pathway. These advantages and capabilities reveal the selfassembled silica nano-web as a HeLa-specific template with an indispensable role in developing advanced selective functional nano-biointerface materials. Our findings provide insights into the potential cellular outcomes of human cervical cancer cells as they interface with SNWs.

\subsection{Materials and methods}

\subsubsection{Materials and laser irradiation}

High-quality polished single crystal N-type silicon wafer with crystallographic orientation of (100) and $625 \mu \mathrm{m} \pm 25 \mu \mathrm{m}$ thickness was supplied by University Wafer University Wafer, Boston, Massachusetts, united states. After dicing the wafer into $2 \mathrm{~cm}^{2}$ square-shaped samples, the samples were ultrasonically cleaned in a $50^{\circ} \mathrm{C}$ acetone bath for 15 minutes, followed by rinsing and drying. To synthesize the deposition of the SNCs, these substrates were exposed to a diode-pumped, Yb-doped femtosecond laser beam (Clark-MXR, Inc.; IMPULSE Series ultrashort pulse laser). This laser system is capable of producing central wavelengths of 1040 $\mathrm{nm}$, and the pulse width can be varied from 214 fs to $1428 \mathrm{fs}$, and the tunable laser pulse repetition rate can vary between $4 \mathrm{MHz}$ (Low rep. rate) and $26 \mathrm{MHz}$ (High rep. rate). A maximum operating average power of $16 \mathrm{~W}$ is attainable. Utilizing ultrashort pulsed laser (USPL) (femtosecond range) is advantageous because the absorption of multiphoton excitation energy delivers the required energy for ionization and provides a localized laser beam spot within the focal point, resulting in precise control of the deposition pattern and area. Samples were therefore irradiated at the beam focal point, and a computerized Galvano scanner was used to precisely implement the synthesis processes. The self-assembled silica nanostructures were synthesized by a single-step femtosecond laser processing of silicon substrate. For the purpose of this study, we fabricated the self-assembled silica nanostructures at pulse widths of 214,714 and $1428 \mathrm{fs}$ and repetition rates of 4, 12 and $26 \mathrm{MHz}$ with laser fluence of 1.57, 3.39 and 10.18 $\mathrm{J} / \mathrm{cm}^{2}$ in order to achieve high, medium and low packing indices of synthesized nanostructures, 
respectively (laser power of $10 \mathrm{~W}$ and Gaussian $1 / \mathrm{e}^{2}$ beam diameter of $10 \mu \mathrm{m}$ ). Scanning speed was constant at $2 \mathrm{~mm} / \mathrm{s}$.

\subsubsection{Cell culture and seeding}

Experimental samples were sterilized under UV light for 20 minutes prior to seeding the cells. Fibroblasts (NIH3T3) and human cervical cancer cell line (HeLa, ATCC, American Type Culture Collection, and ATCC No. CCL-2) were employed in cell experiments to ascertain the comparative functionality of mammalian and cancer cell lines in response to fabricated samples. Fibroblast cells were grown in DMEM containing 10\% heat-activated fetal bovine serum with $1 \%$ penicillin-streptomycin antibiotics (Pen-strep). HeLa cells were grown in DMEM-F12 supplemented with $10 \%$ fetal bovine serum and 1\% Pen-strep. Subsequently, the cells were separately cultured on the substrates placed in Petri dishes with a seeding density of 750,000 cells $/ \mathrm{cm}^{2}$ of substrate surface area. The Petri dishes were placed in an incubator for 24 and 48 hours at $37^{\circ} \mathrm{C}$. Following the study of the cellular response to the nanostructures, the morphology and adhesion characteristics of cells were studied via SEM and FM.

\subsubsection{Cell imaging and morphology}

The cellular morphology of cells seeded on samples was observed using a Hitachi SU1510 scanning electron microscope. After the prescribed time period, spent media were aspirated. This was followed by the fixing of samples in $2 \%$ glutaraldehyde in $0.1 \mathrm{M}$ pH 7.3 sodium cacodylate buffer for 1 hour. Next, the samples were immersed in $0.1 \mathrm{M}$ sodium cacodylate buffer with $0.2 \mathrm{M} \mathrm{pH} 7.3$ sucrose for 20 minutes, followed by dehydration at increasing concentrations of alcohol for 20 minutes. The samples were then critical point dried and coated by gold, at this point cells were ready to be directly observed using SEM.

\subsubsection{Fluorescence microscopy}

Cell adhesion characteristics were also studied using FM. For FM, the samples are first fixed in methanol-free paraformaldehyde followed by incubation in skim milk to prevent non-specific 
binding. To stain the actin and cytoskeleton, the samples were incubated with Alexa Fluor 488 (Life Technologies) followed by DAPI (Life Technologies) to stain the nucleus. The samples were studied using a fluorescence microscope (Nikon, Canada).

\subsubsection{Morphology and physicochemical characterization}

Ultra-high resolution Field emission SEM (UHR-FE-SEM) and energy-dispersive X-ray spectroscopy (EDX) (Hitachi SU8200) were employed to characterize the surface morphology and element analysis of the self-assembled silica nanostructures deposited on the samples. Nanoclusters of nanoparticles were examined in terms of crystallinity and particle size distribution using high-resolution transmission electron microscopy (HR-TEM) (JEOL JEM2010). To separate the nanostructures from the templates for HR-TEM analysis, we immersed the samples in an isopropyl alcohol solution and ultrasonically vibrated the solutions. A drop of the solution was then placed on a grid and dried in a desiccator. (The overall number of particles counted to plot the size distribution histogram were over 1000 particles).To characterize the crystallinity of silica clusters, the diffraction pattern was obtained. An ultra-high-resolution scanning electron microscope was also employed for the morphological characterization and to determine the packing index of nanostructures made of self-assembled silica clusters. Packing index was defined as nanoparticle size distribution and nanogap size distribution for each ablation area. Analysis of images obtained from HR-TEM and HR-SEM, using image processing software ImageJ, revealed three classifications of low, medium, and high structural packing indices. Further elemental analytical techniques were employed using Oxford EDX to identify the elemental changes as a result of silicon oxidation. Moreover, this technique was exploited to characterize the SNC uptakes caused by endocytosis. Compression of oxygen stoichiometry of cells on controls with cells in the presence of SNCs determined by elemental mapping elucidated the apoptotic behavior of the HeLa cell line. 


\subsubsection{Micro-Raman spectroscopy $(\mu \mathrm{RS})$}

Although micro-Raman spectroscopy has been primarily used in chemical composition studies as a complementary technique to other methods, deducing crystallinity information is advantageous to this technique as a non-destructive method to determine the formation of amorphous silica. Raman spectroscopy was used to quantify the relative amounts of amorphous and crystalline silica in deposits. By mapping an area of deposited silica, the uniformity of the distribution of the two amorphous and crystalline was observed. Raman spectra were obtained using a Bruker SENTERRA dispersive Raman microscope with a central wavelength of $532 \mathrm{~nm}$ as the excitation source. This is an application for which Raman spectroscopy is particularly well suited. Silicon-silicon bonds are symmetrical and result in strong Raman scattering. Crystalline silicon has highly uniform bond angles and bond lengths and exists in a limited number of states.

\section{Statistics}

All cell-based assays were performed at least three times, and the data points are averages unless otherwise noted. The error bars indicate the mean \pm standard deviation (SD). Statistical significance was evaluated using one-way analysis of variance (ANOVA), with *p $<0.05$ and **p $<0.01$ suggesting significant difference.

\section{Appendix 1}

Appendix 1, details about HeLa cell interaction with freestanding SNWs after 24 hours of incubation. Appendix 2, further characterizations of Hybrid-SNWs including morphological, crystallographic analysis and histogram of particle size distribution. 


\subsection{Results and discussion}

\subsubsection{Fabrication and characterization}

The nano-biointerface templates were synthesized by single-step femtosecond laser processing of silicon ( $\mathrm{Si}$ ) chips in atmospheric conditions, as depicted in the schematic in Figure 4.1. A computerized Galvano scanner laser enabled precise 2D (X-Y) movement of the laser beam on the $\mathrm{Si}$ chip surface. Therefore, pre-designed patterns could be transferred onto the surface of the $\mathrm{Si}$ samples.

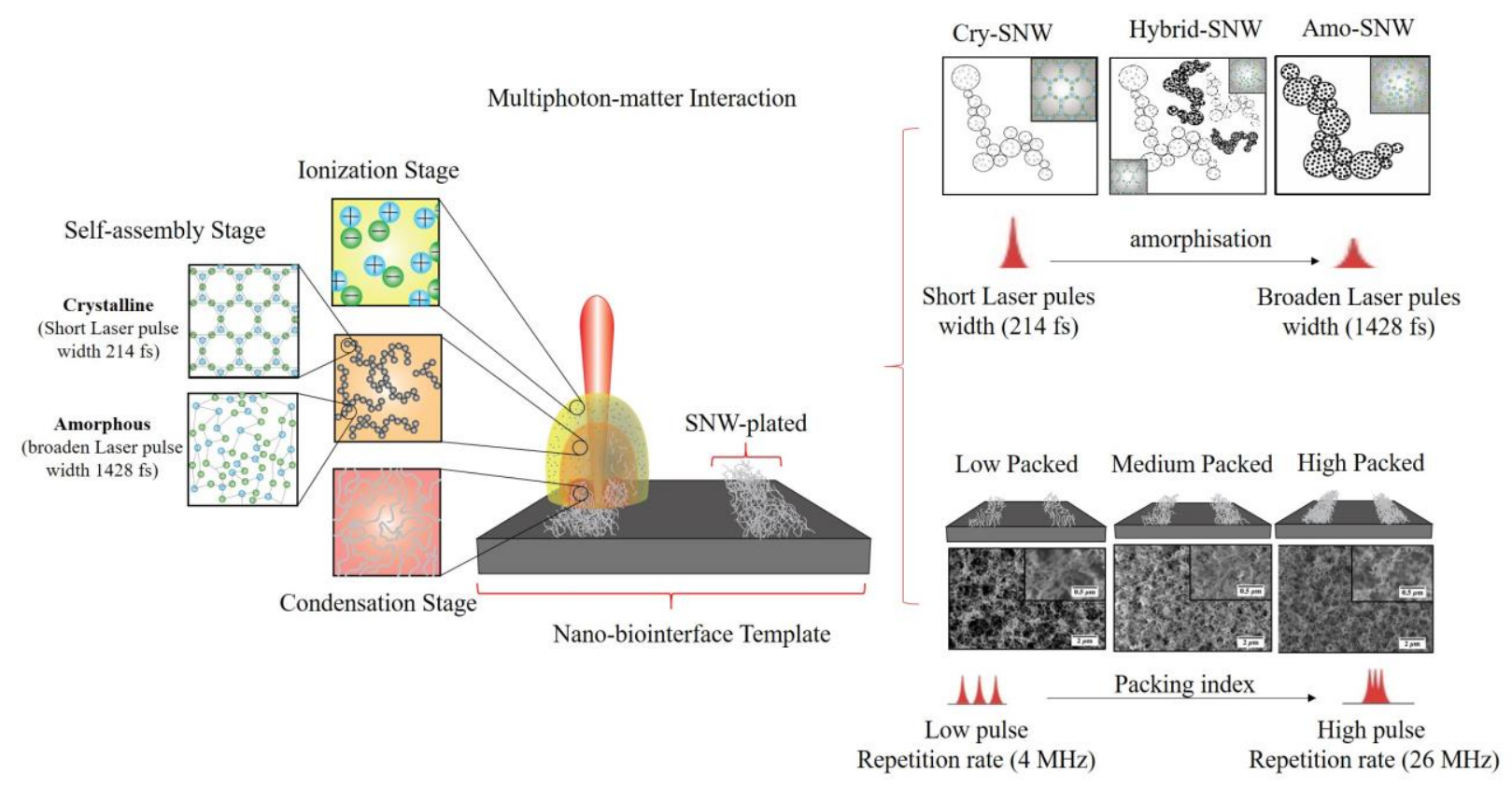

Figure 4.1: Schematic illustration showing the one-step process of the SNW-plated template. Multiphoton-matter interaction towards the synthesis of the SNW is governed by the laser pulse repetition rate and laser pulse width on which packing index and crystallinity of SNWs plated on the template can be precisely modulated.

The laser processing of the Si chips at megahertz $(\mathrm{MHz})$ pulse repetition rates triggers surface ablation, evaporation, and the formation of the plume, which is composed of ionized $\mathrm{Si}$ and air molecules. Expansion of the plume in the background air causes condensation as a result of temperature dissipation, leading to the formation of the nucleus and growth of self-assembled silica nanoclusters. The nanoclusters after plume condensation form a deposition of 
accumulated nanowebs on the Si samples. The packing index of synthesized silica nanowebs is a function of self-assembled nanoparticle size variation during the ionization process.

The most influential laser parameters by which these attributes have been governed are laser repetition rate and pulse duration. Increasing the increment of the repetition rate from $4 \mathrm{MHz}$ to $26 \mathrm{MHz}$ decreases the average nanoparticle size ${ }^{42}$. Furthermore, the increment of the repetition rate has a direct relationship with the yield of the synthesized nanoweb. In other words, the increment of laser fluence, which is the energy delivered per unit area during the exposure of the Si sample to the laser beam, intensifies the tendency of ion species to diffuse and grow the nuclei.

Moreover, the average area of vacuities of the nanoweb, measured using image analysis software, was defined as a packing index of synthesized nanomaterials. Reduction of nanoparticle size along with the increment of yield materials contributed to closely pack synthesized nanostructures with an average area of $3.1,2.5$, and $0.9 \mathrm{~nm}^{2}$, respectively, for 4, 12, and $26 \mathrm{MHz}$. Therefore, ionization energy governed by laser fluence has an analogous influence on the packing index of the condensed silica nanoweb.

Additionally, prolonging the laser pulse from 214 fs to $1428 \mathrm{fs}$ showed the predominance of the amorphous phase of the silica structure, in which the ionized molecular fragments were desorbed, believed to be caused of amorphization ${ }^{43-46}$. As shown in Figure 4.1 an intermediate laser pulse width (714 fs) has resulted in formation of hybrid-SNWs which contains both Cry and Amo silica structure. However, High resolution FE-SEM revealed that laser repetition rate has an equal influence on packing index of the synthesized SNWs. The HR-TEM diffraction pattern (Figure 4.2 (C)) also confirmed the formation of amorphous silica, as shown in Figure $4.2(\mathrm{c} 2)$. 


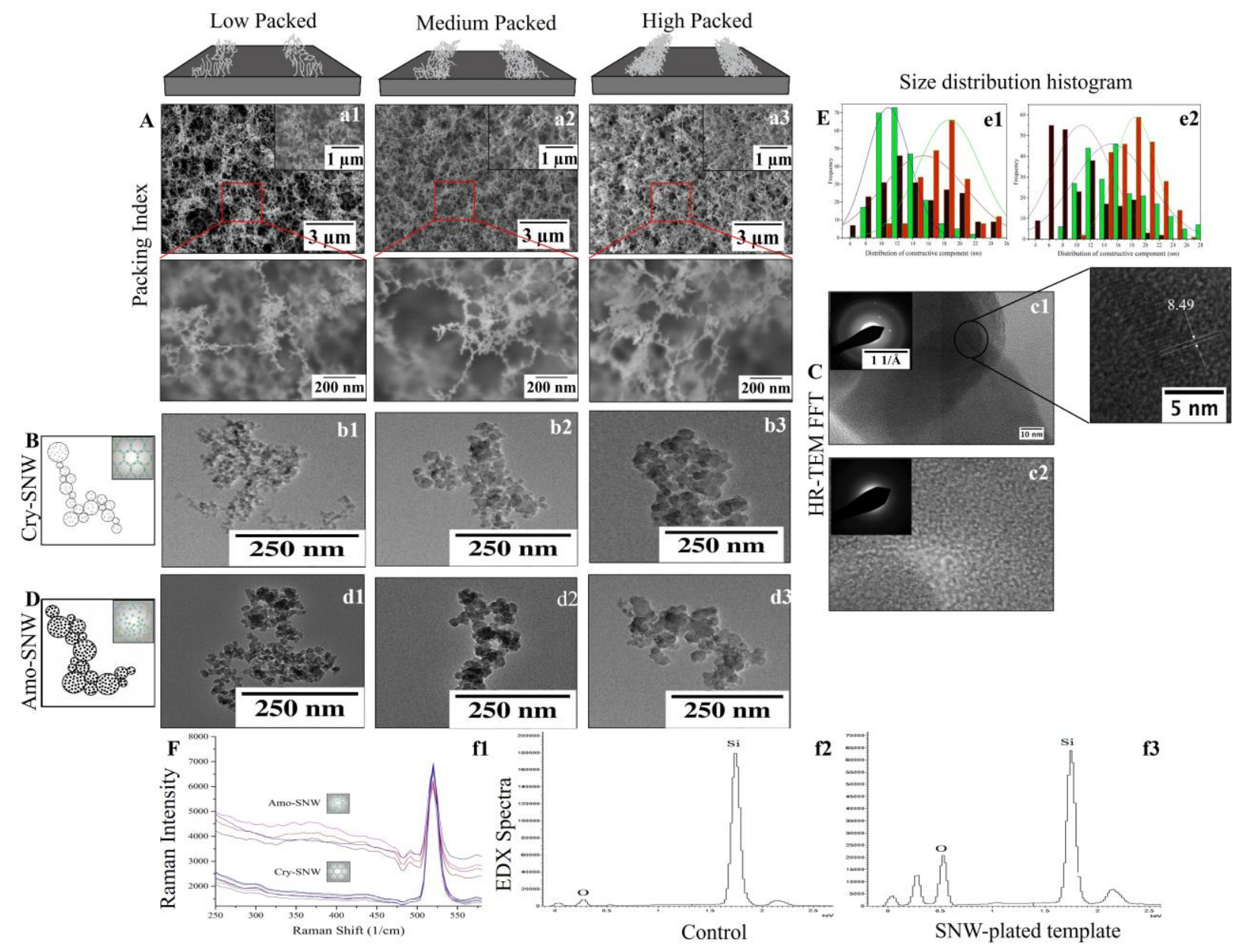

Figure 4.2: UHR-FE-SEM micrographs in A (a1-a3) illustrate low-packed, medium-packed and high-packed SNWs. HR-TEM images show the Cry-SNW (b1-b3) and Amo-SNW (d1-d3) with respect to their packing index. Size distribution of the constructive component of Cry- and Amo-SNWs plotted in histograms E (e1 and e2), respectively (Statistical analysis done over 1000 particles, with Stan. dev. 0.63). The HR-TEM crystal d-spacing and diffraction patterns demonstrated in image (c1c2) show the crystallinity of the synthesized SNW. Raman spectra (f1) exhibit the crystallinity changes of the SNW. EDX taken from control and SNW-plated template represent the presence of oxygen because of silica formation (f2 and f3).

Furthermore, the disturbed plume during atmospheric laser-matter interaction contains many species, i.e., ionized substrate molecules (anions and cations of the silicon matrix molecules) as well as neutral and reactive gases. Hence, reactive oxygen participates in chemical phase transformation through which Si-O bonding occurs. EDX analyses further corroborated the presence of oxygen in all the synthesized SNWs (low, medium, and high packed), as shown in Figure 4.2 (f2 and f3). The use of ultra-short pulsed laser (USPL) has provided the advantages 
of single-step processing of a silica nanoweb through which precisely tuning the laser parameters controls the particle size as well as direct amorphization. In this study, three sets of samples were plated with distinct low, medium and high packing indices for both crystalline and amorphous structures to probe the cellular response to the synthesized SNWs. The SNW plated templates at three different packing indices are demonstrated in 3.2 (A) (a1-a3). HRTEM images show the self-assembled Cry-SNW (b1-b3) and Amo-SNW (d1-d3) with respect to their packing index. Figure (4.2) (E) (e1 and e2) also shows the size distribution variation of the constructive component of Cry- and Amo-SNWs with respect to their packing indices. These data were measured using HR-TEM micrographs and image-processing software. The average size of the nanoparticles was 6,12 and $19 \mathrm{~nm}$ and 9, 15 and $22 \mathrm{~nm}$, respectively, for crystalline and amorphous structures at low, medium and high packing densities. Raman spectra (Figure 4.2 (f1)) showed a strong band and sharp peaks at $521 \mathrm{~cm}^{-1}$, which is characteristic of a crystalline substance. In contrast, amorphous silica that is less oriented in its atomic arrangement exhibited a broadened peak at approximately $480 \mathrm{~cm}^{-1}$ that was readily distinguishable from that of crystalline silicon. Figure (4.2) (f1) shows Raman spectra from samples that range from pure crystalline to those containing considerable amounts of amorphous phase synthesized at 214 and 1428 fs, respectively. A transition from short laser pulsed width to broadened pulse width causes the crystallinity changes from crystalline to hybrid (Cry and Amo) and ultimately amorphous state of silica. The laser pulse width at which both Cry-SNW and Amo-SNW created, has found to be 714fs. The cell response of Hela and fibroblast to the synthesized Hybrid-SNWs has demonstrated a mediatory impact of Hybrid structures on HeLa cells. 


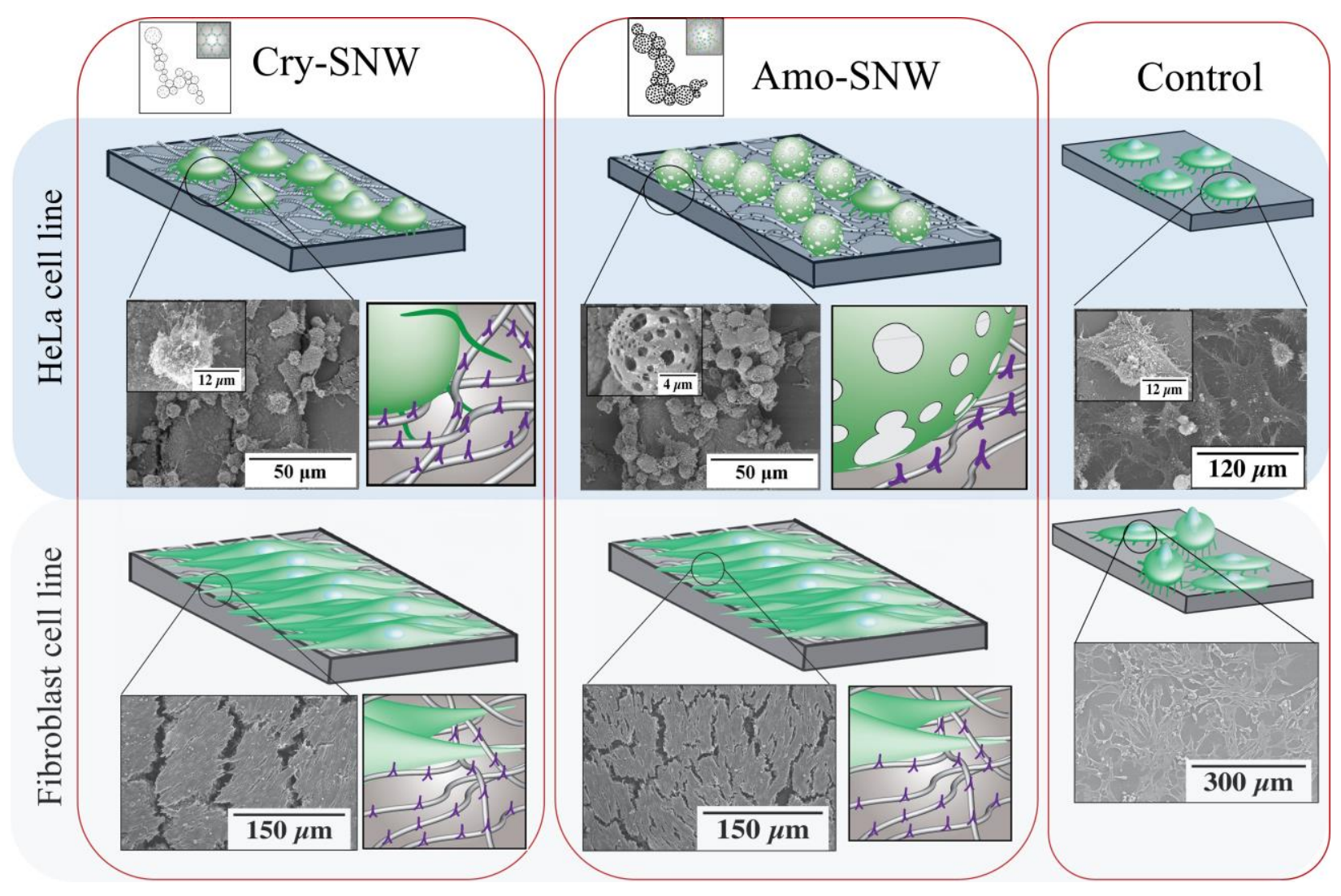

Figure 4.3: Cellular response to the crystallinity changes of SNW demonstrated for HeLa and fibroblast cell lines. Interaction of HeLa cells with the Amo-SNW caused an apoptotic-like pathway. Interaction of fibroblasts with both Amo- and Cry-SNWs resulted in a tissue-like structure. Cellular behavior on native (control) substrate showed normal adhesion and proliferation for both HeLa and fibroblast cells.

\subsubsection{Cellular Response to Silica Nano-webs}

\subsubsection{Influence of the packing index of the SNW - plated template on cellular attraction}

HeLa and fibroblast cells were cultured for 24 and 48 hours on untreated silicon samples (control) and experimental samples fabricated, as described above, to study the attraction, adhesion, and proliferation criteria of cell lines. The results of the control have been taken as a reference for the experiments. Cellular interaction with crystallinity alteration and packing index changes of SNWs were examined in terms of the cell viability, morphology and efficiency of SNWs to attract cells. As demonstrated in Figure 4.3, distinct cellular responses were observed on which the cellular interaction of HeLa cells with the Amo-SNW showed cell 
roundness and cytoskeleton rupture, while the Cry-SNW exhibited only a minor deduction in filopodia extension. Fibroblast cell interaction with both Amo- and Cry-SNWs displayed a contrary behavior in which tissue-like structure as a result of growth enhancement was observed. Remarkably, an analogous tendency of cells to adhere on the SNW-plated template regardless of the crystallinity of SNWs was observed in both cell lines. Figure 4.4 compares the cell number and morphology of fibroblasts and HeLa cells attached to control and experimental templates. Comparisons of SEM and FM micrographs taken from low, medium and highly packed nanostructure, shown in Figure 4.4 (A-B), indicate that HeLa cellular attraction increases as the packing index of the nanostructures increases. As depicted in Figure 4.5 (B) Cytoskeleton expansion of fibroblast cells at constant cell count and identical unit area show higher attraction of cells to SNWs compare to the control after 24 and 48 hours by increment factor of 3. Stretched and extended cellular morphology also suggested that fibroblasts have a greater affinity with the amorphous silica than with the crystalline structure. The fibroblasts seeded on the control sample exhibited a regular shape, smaller size, and random distribution. In contrast, adhered fibroblast cells on the SNW-plated template exhibited a well-ordered distribution within filopodia and actin filaments, especially after 48 hours of incubation. While the number of seeded fibroblast cells increased after 48 hours in the both control and experimental samples, tissue-like morphology was observed on the SNW, as evidenced by SEM imaging in Figure 4.6. Confluent monolayers were formed in a way that made distinguishing a single cell nearly impossible. Thus, highly packed nanostructures of amorphous silica enhanced fibroblast proliferation. Further analysis using FM was performed to study cellular interactions with the SNWs. 


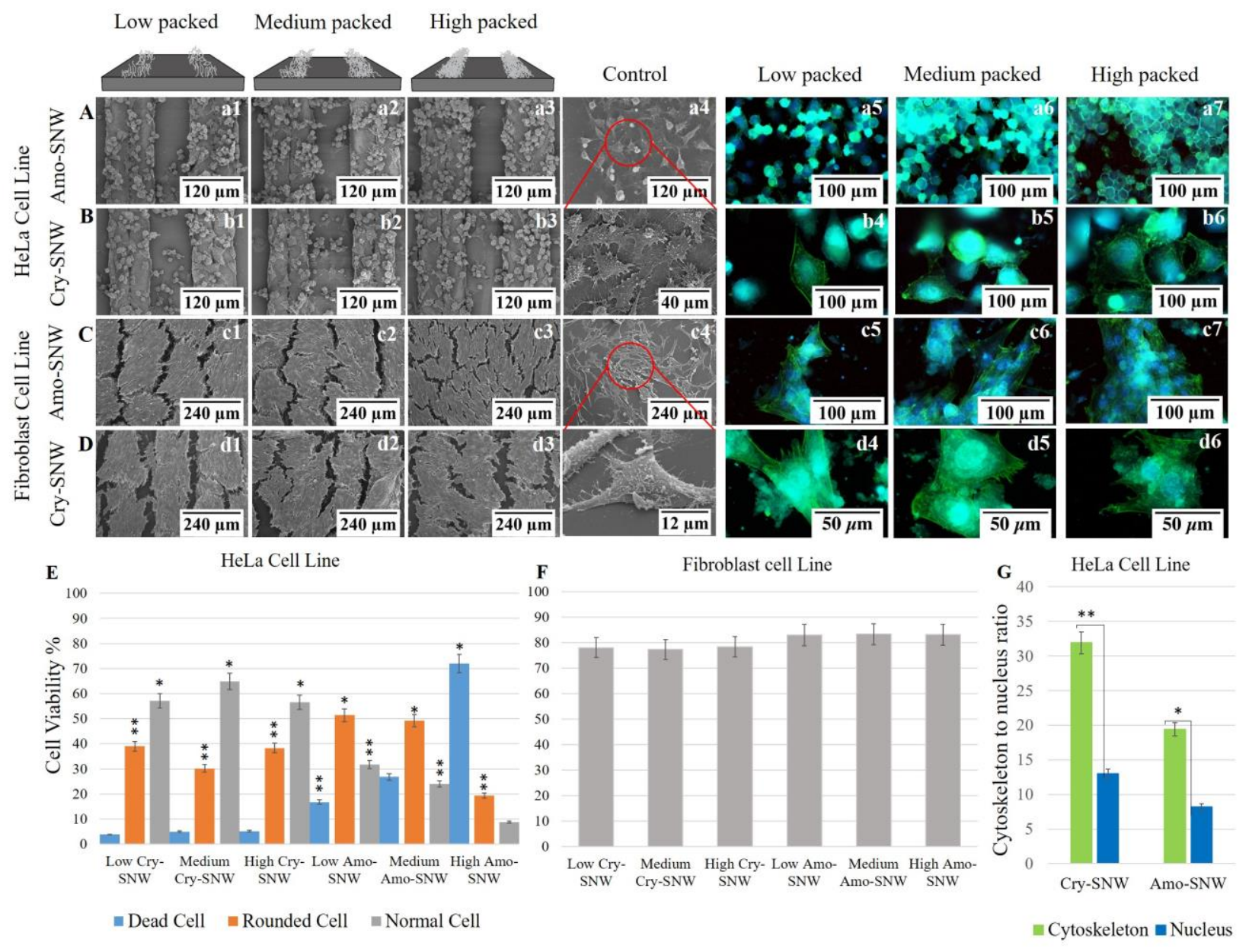

Figure 4.4: Cellular attraction as a function of packing index of SNWs. SEM and FM of HeLa (A-B) and Fibroblast (C-D) cells, respectively, seeded on Amo-SNW (a1-a3, a5-a7 and c1-c3, c5-c7) and Cry-SNW (b1-b3, b4-b6 and d1-d3, d4-d6) show distinctive attraction characteristics with respect to the packing index of the SNW. Corresponding FM images are also in accordance with SEM micrographs in which attraction of the cells to the SNW increases for highly packed nanowebs. Control samples show random orientation and growth of HeLa (a4) and fibroblast cells (c4). A cell viability assay of HeLa cells (E) shows the increase of dead cells on the Amo-SNW along with an increasing packing index. Fibroblast exhibits average of 80 percent cell viability (F). Cytoskeleton to nucleus ratio comparison (G) based on fluorescent imaging revealed the cell shrinkage and nucleus size retraction as HeLa cells interacted with the Amo-SNW. Error bars show the standard error of the mean; two independent experiments were repeated with $n=3$ in each experiment. Statistical significance is shown with $* p<0.05$, $* * \mathrm{p}<0.01$ (data presented in graphs are in percentages).

The cellular behavior of HeLa cells and fibroblasts in the presence of SNWs and in control samples is shown in Figure 4.4 (A-D). The findings from the study of fluorescence were in agreement with our SEM observation of the cell morphology and adhesion mechanism. The 
morphology of cells seeded on the control sample (Figure 4.4 (a4,c4)) revealed a random orientation of grown cells with well-defined and flattened cellular organisms, numerous filopodia extensions, and focal adhesion points, which are cell-substrate interaction points. Moreover, the presence of randomly oriented stress fibers indicates the random arrangement of cells on the control ${ }^{47-49}$. A comparison of the cytoskeleton to nucleus ratio (Figure $4.4 \mathrm{G}$ ) and the number of stress fibers between the control and the experimental groups were reduced, which indicates that the cells are apoptotic ${ }^{50}$.

A

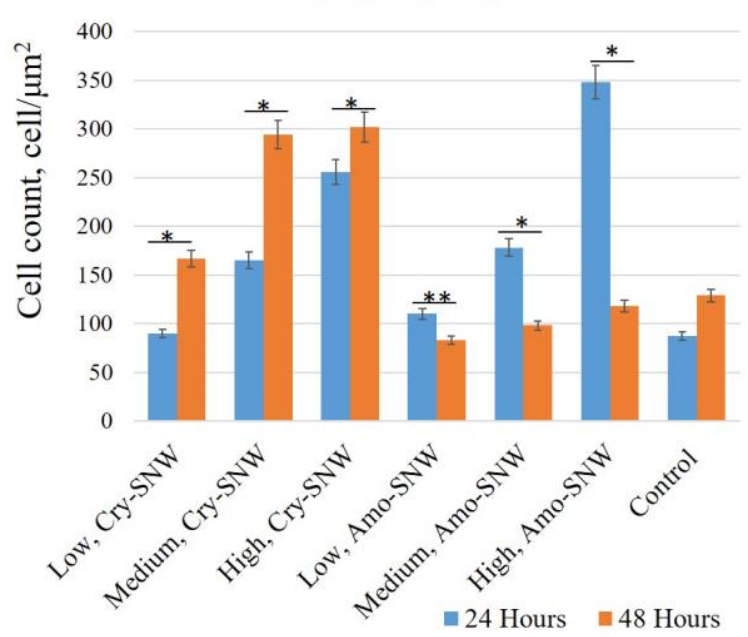

B

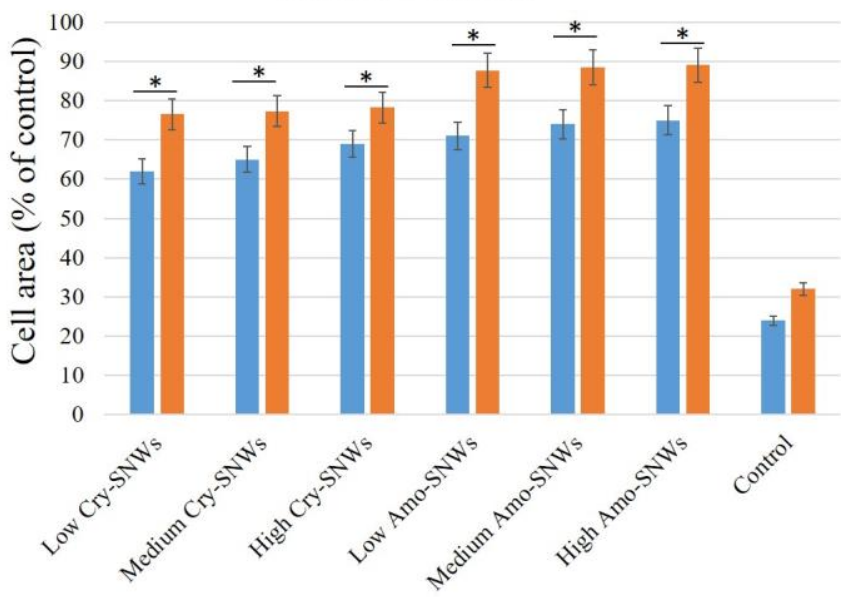

Figure 4.5: Statistical analysis based on the cell number per unit area (A) demonstrated that HeLa cells after 24 and 48 hours seeded on low, medium and high packed SNWs for both Amo- and Cry-SNW. Cytoskeleton expansion of fibroblast cells (B) at constant cell count and identical unit area show higher attraction of cells to SNWs compare to the control after 24 and 48 hours. Error bars show the standard error of the mean. Two independent experiments were repeated with $n=3$ in each experiment. Statistical significance is shown with $* \mathrm{p}<0.05, *{ }^{*} \mathrm{p}<0.01$ (data presented in graphs are shown as percentages).

In vitro interaction of cervical cancer cells with polychromic SNWs was assessed using HeLa cell lines after 24 and 48 hours of incubation. The response of HeLa cells to these SNWs revealed distinctive functionalities, in which the packing index and crystallinity of SNW-plated templates played remarkable roles. First, the influence of the packing index was investigated in terms of cellular attraction to the nanowebs, which is a primary step for cell adhesion. It was observed that the packing index of nanowebs has a notable effect on the modulation of cellular attraction. At 24 hours, significantly more cells had attached to the high-packed SNW compared 
to the low-packed SNW, which was comparable between HeLa and fibroblast cells. As the cell count indicated in Figure 4.5 (A), cell adhesion as a consequence of protein adsorption and topographical changes was significantly multiplied. This increased cell attraction was predominant in highly packed nanowebs, which were composed of a relatively smaller SNC size. Cell affinity is believed to be the result of simultaneous changes in physical and chemical aspects of the deposited nanostructures. Cells are profoundly influenced by surface roughness, and many cell types can sense topographical changes well into the $\mathrm{nm}$ range ${ }^{51-53}$. Moreover, it is conceivable that a decrease in nanoscale size has a drastic influence on surface area. It has been postulated that the increase of surface area on a highly packed SNW, which causes intensification of protein adsorption, along with topographical changes, is the initial stimulus of cell attraction ${ }^{54-57}$. Upon immersion of the samples in cell culture medium, nanostructures adsorb the protein content in the FBS prior to the commencement of cell adhesion ${ }^{58-59}$. Cell adhesion is therefore governed by protein type. Thus, different proteins (e.g., albumin, fibronectin, and vitronectin) play major roles in cellular attraction ${ }^{60}$.

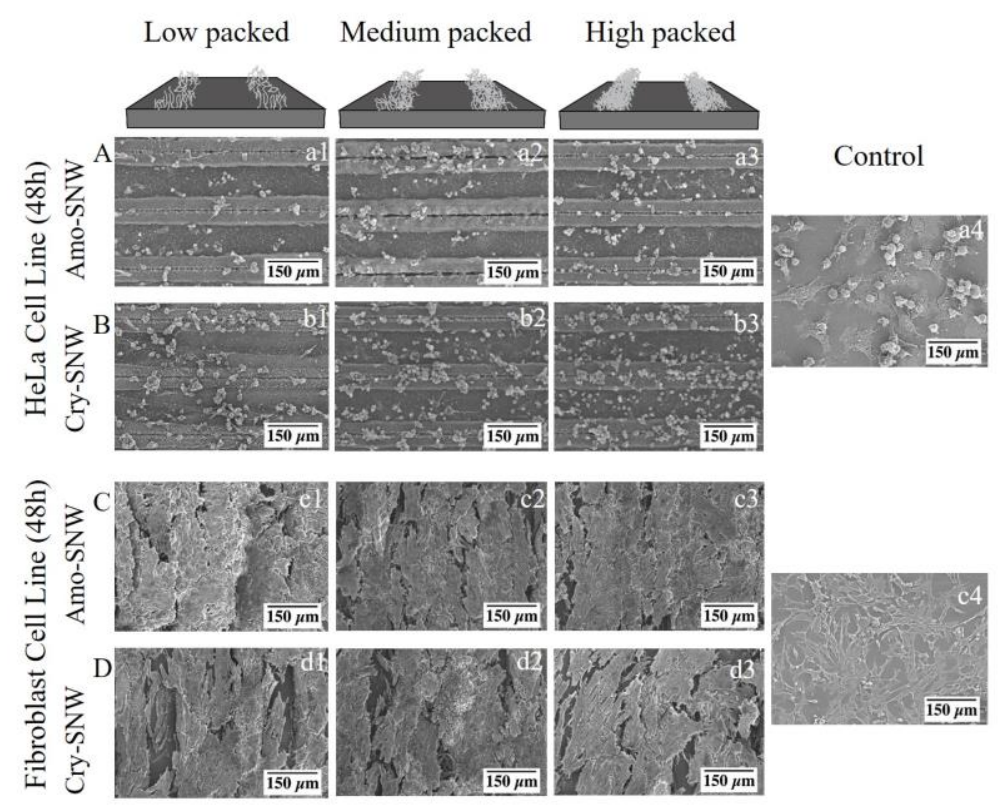

Figure 4.6: Cellular response to the SNW after 48 hours. SEM micrographs of HeLa cells (A-B) and fibroblasts (C-D) after 48 hours of incubation show that the number of HeLa cells on Amo-SNWs (a1-a3) decreased. However, cells on Cry-SNWs (b1-b3) exhibited a proliferative behavior. Fibroblast cells showed tissue-like structures on both Amo- and Cry-SNWs (c1-c3, d1-d3). (a4,c4) show HeLa and fibroblast cells seeded on control sample. 
It is believed that fibronectin and vitronectin - known cell-adhesive serum proteins - are the key elements mediating the cellular attraction to the nanostructures, and hence, cell adhesion ${ }^{52,61}$. Depending on the physicochemical property of absorbance, in this case of the nanowebs, the packing index was one of the persuasive functions driving cellular attraction. For HeLa cell lines, SNWs with higher packing indices showed a greater degree of cellular attraction than did those with lower indices. This attractive behavior was also exhibited in the fibroblast cells. The proliferation and health of the fibroblast and HeLa cells were further analyzed based on silica polymorphism. After 48 hours of incubation and cell growth, there were drastic changes in cell attachment characteristics of the HeLa cell line with respect to the crystallinity of SNWs (presented in Figure 4.6), where Amo-SNWs resulted in a drastic reduction in cell number.

\subsubsection{Intracellular reaction to the polymorphic alteration of SNWs}

The cellular response of HeLa and fibroblast cell lines to packing index was found to be similar. However, contradicting responses of cells to crystallinity were also observed. While the fibroblast cells grew and spread well on both the Cry- and Amo-SNWs, HeLa cells showed an apoptotic-like response to the Amo-SNWs. Moreover, the attractive feature of closely packed nanowebs enhanced apoptotic efficiency. Morphological assessment of HeLa cells seeded on both Cry- and Amo-SNW-plated templates revealed explicit changes to the cytoskeleton. This observation showed that in comparison to the Cry-SNWs, the number of rounded cells increased drastically on Amo-SNWs, and amorphization of the synthesized silica nanoweb was achieved via the extended multi-photon matter interaction. As the statistical assessment of cell viability in Figure 4.4 (E) shows, the number of dead cells per unit area dramatically increased on the Amo-SNWs. While viability assessment of fibroblast cells (Figure 4.4 (F)) seeded on low, medium and high packed of Cry-SNWs and Amo-SNWs exhibited the average of 80 percent cell visibility which is comparable with the control sample. However, distinctive morphological changes imply proliferative characteristic of fibroblast cells in presence of the SNWs. The results of 24 hours of incubation of HeLa cells on closely packed Amo-SNWs indicated 71.1\% apoptotic cells, $19.9 \%$ rounded cells and only $8.9 \%$ cells of normal morphology. Seeded cells on Cry-SNWs with similar packing indices showed approximately 6\% apoptotic cells, 56\% rounded cells and $38 \%$ normal cells. Additionally, a drastic decrease in the cell number of Amo- 
SNWs was observed after 48 hours, as SEM images show in Figure 4.6 (A). This disparity in the reduction of cell number can be attributed to the apoptosis pathway induced by endocytosis of Amo-SNCs through which dead cells lose their adhesion to the nanoweb and become detached. Cry-SNWs, however, exhibited an opposite trend in which cell count had increased after 48 hours. The apoptotic pathway induced by endocytosis in HeLa cells is illustrated in Figure 4.7.
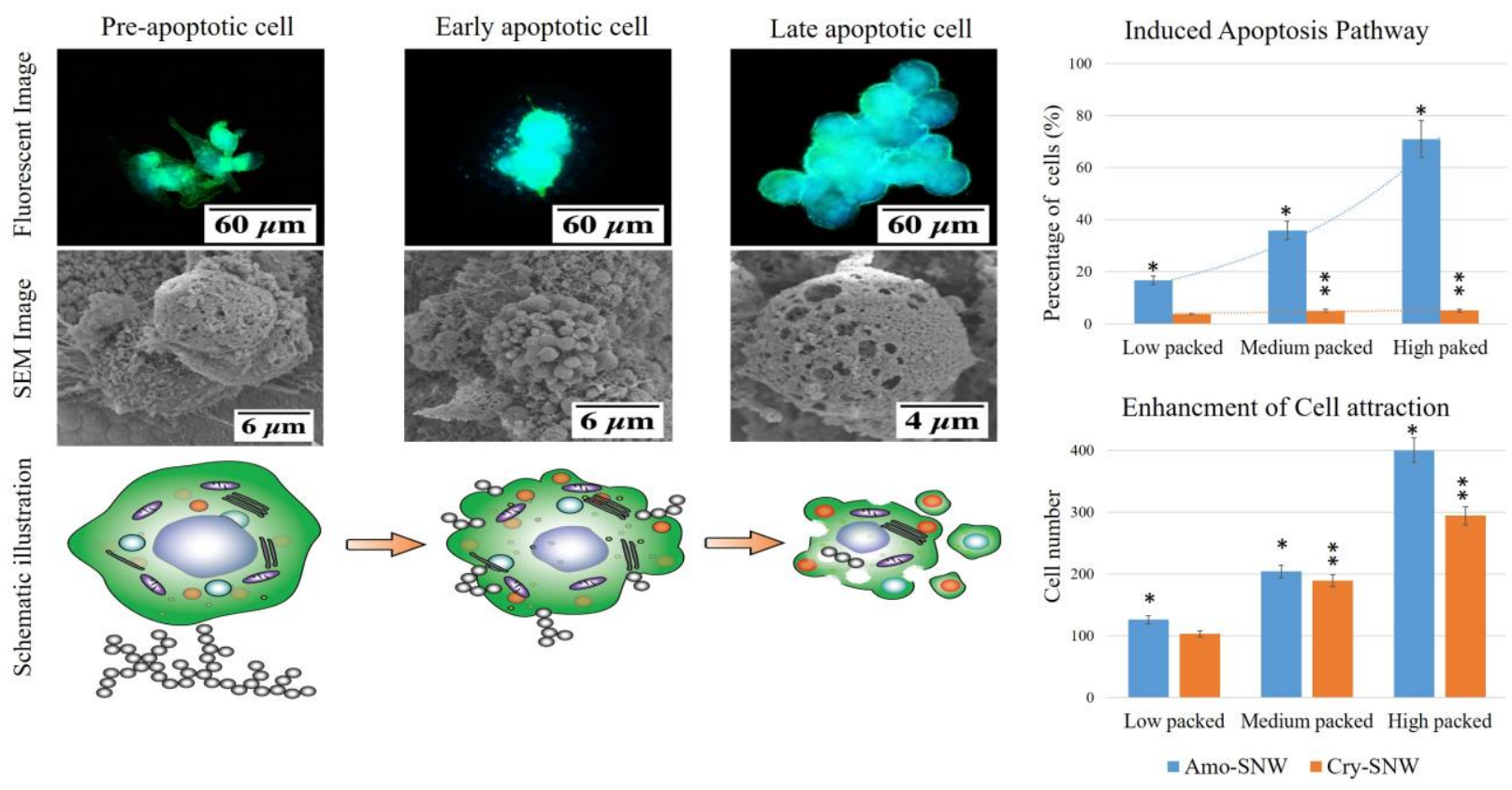

Figure 4.7: Demonstration of the interaction of HeLa cells with the SNWs. FM and SEM imaging illustrate that endocytosis of amorphous SNCs is the cause of cell death. The sequence of the apoptosis-induced pathway is also schematically shown. The graph on the top left indicates the efficiency of the induced apoptosis pathway by Amo- and Cry-SNWs. The graph on the left bottom shows the enhancement of cellular attraction as a function of packing index. Error bars show standard error of the mean. Two independent experiments were repeated with $n=3$ in each experiment. Statistical significance is shown with $* \mathrm{p}<$ $0.05, * * \mathrm{p}<0.01$ (data presented in graphs are in percentages).

During the incubation period, cells preferentially attach to areas of higher protein adsorption, i.e., the closely packed nanoweb. After initial attachment, it is hypothesized that the contiguity of cells to the Amo-SNWs causes the uptake of constructive components of the nanowebs, namely, SNCs. This interaction leads to a loss of cellular integrity, and thus, the potential for growth is greatly hindered ${ }^{39,62-64}$. HeLa cells seeded on Cry-SNWs after 24 hours showed the least cell death but greater cell proliferation after 48 hours. Therefore, apoptosis in HeLa cells 
was induced by the uptake of Amo-SNCs. As presented in Figure 4.7, the interaction of HeLa with both the SNW-plated template and freestanding SNWs caused the rounding of the cytoskeleton, a drastic reduction in size, and a lack of filopodia extensions. Cell roundness is believed to be a stage preceding cell death through the apoptosis pathway. However, the rounded shape of cells could either be due to the mitosis that cells undergo during cell division or an early stage of the apoptosis pathway in which the cell membrane shrinks and forms a spherical shape. To better differentiate these two phenomena, 48 hours of cell incubation was performed. Cellular deduction on the Amo-SNWs eliminated the possibility of proliferation through cell division. The cellular attraction characteristic of the closely packed SNWs was shown to augment this attribute. By using EDX to trace the existence of SNCs in the cell cytoskeleton, the intrinsic pathway that was activated upon the uptake of SNCs into the cell membrane could be detected. Elemental mapping of cells seeded on the control sample and in the presence of the Amo-SNW, as shown in Figure 4.8 (A and D), elucidates the oxygen present due to Si-O bounding (a2 and c2). It has been shown many times that cancer cells have an intrinsic tendency to migrate to other areas and metastasize ${ }^{65-66}$. Due to this attribute, we hypothesize that the attractive characteristics of the SNW-plated template that immobilize the adhered cells, along with endocytosis, are primary inducers of the apoptosis pathway.

\subsubsection{Cell response to the freestanding SNWs}

After synthesis of the same types of SNWs, these SNWs were suspended in the cell culture medium in order to rule out the influence of cell immobilization. Thus, cellular interaction with suspended SNWs was investigated to eliminate the influence of immobilization through the attractive characteristic of plated template and, in particular, to study the apoptosis of HeLa cells through several different endocytic pathways ${ }^{63,67-69}$. Although the attractiveness of the SNW-plated template to cells dramatically enhanced the proliferation of fibroblasts and the apoptosis of HeLa cells, it is understood that suspended Amo-SNWs have an identical apoptotic effect on HeLa cell behavior. Notably, a significant enhancement of apoptotic properties was observed as the size of the constitutive components of Amo-SNWs decreased from $22 \mathrm{~nm}$ to 9 $\mathrm{nm}$. Utilization of SEM imaging to investigate the cellular morphology confirmed that porous round HeLa cells underwent the apoptosis pathway. As a result of the penetration of nanoparticles 
into the nucleus, the damaged cell causes cytoskeleton shrinkage, which is the initiation of cell death, as evidenced in SEM and FM images in Figure 4.7 (a1 and a5). In addition, EDX analysis showed a trace of SNCs in the cytoskeleton of cells (Figure 4.7(a2-a3)) which is in accordance with FM image (Figure 4.7(a4-a5)) where accumulation of SNCs are evident in bright dots (Figure 4.7(a5)). In spite of the fact that presence of oxygen is an inevitable in organic substance, not only higher concentration of oxygen as a result of SNCs uptake can be distinguished by compression of the cells seeded on control and the ones with suspended SNCs, but also presence of silicon element manifests the uptake of SNCs. Taken together the EXD and FM results from HeLa and fibroblast led to a conclusion that induced apoptosis pathway to the Hela cells is due to uptake of Amo-SNCs while fibroblast cells did not show a trace of uptake (Figure 4.8 (C-D)).

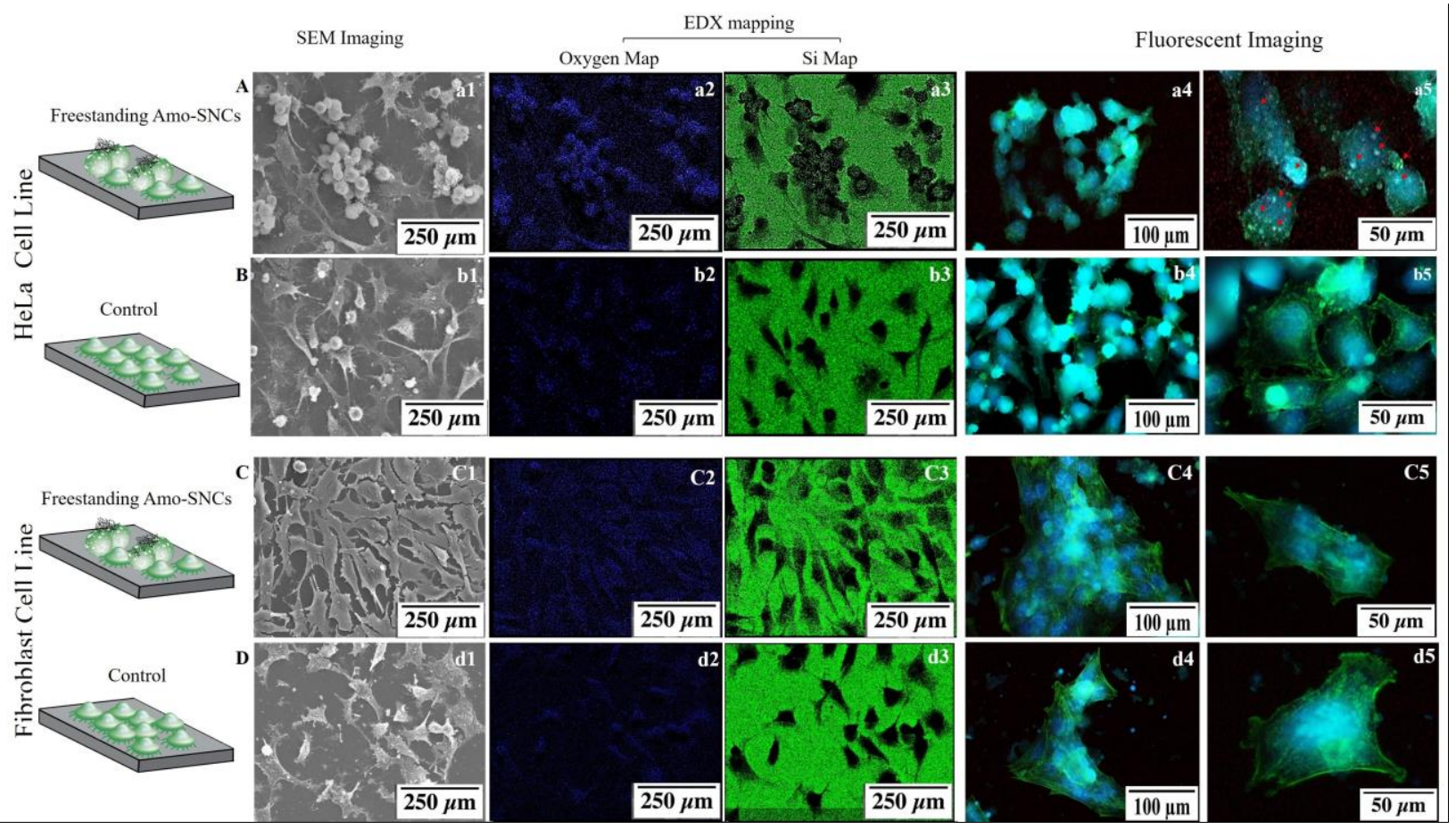

Figure 4.8: HeLa cells in the presence of suspended SNCs (a1-a5); SEM image (a1) and EDX elemental mapping of oxygen (blue) and silicon (green) (a2-a3) and fluorescent imaging (a4-a5) represent the SNCs penetrated into the cell (red arrow markers indicate uptake of SNCs by the HeLa cells). HeLa cells on the control sample (b1-b5); SEM imaging and EDX mapping on (b1, b2-b3), where there is no trace of SNCs. Fluorescent image of HeLa cells in the control sample (b4-b5). SEM and FM images of fibroblast cells in presence of suspended SNCs (c1, c4-c5) show enhancement of proliferation behavior compare to the cells on the control (d1, d4-d5). EXD analysis of fibroblast in presence of suspended SNCs (c2-c3) compare to the control (d2-d3) do not indicate higher concentration of oxygen and hence uptake of SNCs. (Minimal trace of oxygen in either cell lines are due to substantial nature of organic substance). 
Furthermore, we hypothesized that altered cytoskeleton size and reduction of nucleus size in HeLa cells in the presence of the SNW might cause damage to chromatin organization and gene expression $^{70}$. The inhibition of apoptosis that is caused by proteins in HeLa cells is partially responsible for the immortality of HeLa cells and is believed to be the main driver of resistance to chemotherapy and common therapeutic methods ${ }^{71-72}$. These Amo-SNWs have shown the ability to induce an apoptosis pathway despite this inhibiting feature of the HeLa cell line. This endocytic property of Amo-SNWs provides promising insight into the development of an alternative treatment for cervical cancer cells. In opposition to other research studies that utilize labeled nanoparticles as a logistic or carrier to deliver drugs, this study reveals an attractive characteristic of nanowebs that effectively increases the therapeutic function of a synthesized polymorphic SNW. There is an approximately 4 -fold higher efficiency at attracting cells by reducing the packing index of SNWs and the emergence of an apoptosis pathway as a result of silica amorphization. The appendix 1 shows details about HeLa cell interaction with freestanding SNWs after 24 hours of incubation.

\subsubsection{Cellular response to the hybrid polymorphic SNWs}

In the second part of the study, we examined the effects of the hybrid crystalline/amorphous nanoweb on HeLa and fibroblast cell behavior. The SEM images presented in Figure 4.8 show that cellular attraction has a direct relationship with the packing index of SNWs, while the crystallinity still plays a major role in the cellular response. The fibroblast cell interaction with the hybrid nanoweb of an amorphous/crystalline nature exhibited a proliferative response after 24 hours and tissue-like behavior after 48 hours, while HeLa cells showed a minimally induced apoptosis pathway. This study found that by regulating the proportional relation of the crystalline/amorphous structure, cell behavior can be modulated, prolonging the apoptotic response of HeLa cells. 


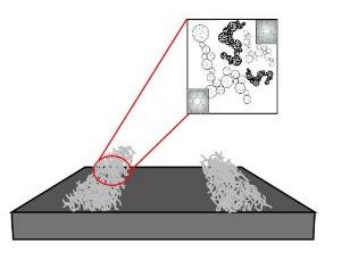

Hybrid SNW-plated template
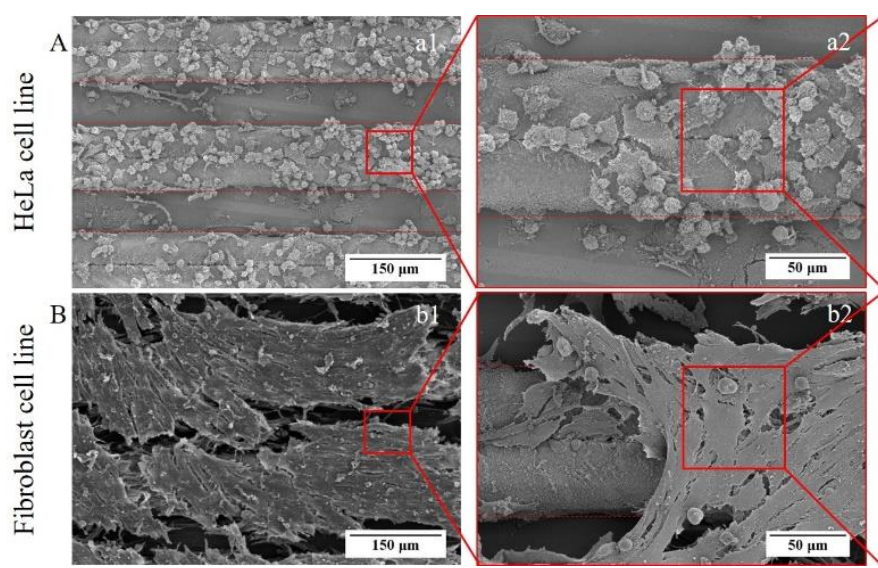

Figure 4.9: Cellular interaction with hybrid SNW-plated (Amo and Cry) template exhibited by HeLa and fibroblast cell lines. SEM images of HeLa (A) and fibroblast (B) cells in the presence of hybrid SNWs. HeLa cells exhibited an attraction to the hybrid SNW-plated template; magnified images (a1-a3) show proliferative and apoptotic behavior. Fibroblast cells exhibited an affinity for hybrid SNWs (b1-b3) (dashed lines indicate the boundaries of plated zones).

Hybrid SNWs composed of both crystalline and amorphous structures were synthesized at an intermediate laser pulse width of 714 fs. The SEM images (Figure 4.8) show that the efficiency of these nano-webs to attract cells remained the same, regardless of the associated crystallinity. However, HeLa cells exhibited a rounded and ruptured cytoskeleton, while the number of apoptotic HeLa cells was not comparable with that observed in fully Amo-SNWs. Further characterizations of hybrid SNWs including morphological, crystallographic analysis and particle size distribution histogram provided in appendix 2.

\subsection{Summary}

Silicon-based nanomaterial has broadened the frontiers of cancer therapy through drug delivery, cell regulation, and advancements in cell imaging. However, to the best of our knowledge, the functionalization of dormant silicon via multiphoton ionization to the SNW with HeLa centric characteristic has never been reported. This in-vitro study probed the interaction of HeLa cancer cells and mammalian fibroblast cells with a polymorphic self-assembled SNWs tailored to simultaneously take advantage of the therapeutic attributes of SNPs and the attraction characteristics of the ECM, without the need of its conjugation with a biomarker. This research focused on the enhancement of cellular attraction and selective functionality through which 
HeLa cells showed induction of apoptosis-like pathways, and fibroblasts exhibited tissue-like behavior. Plated-SNWs demonstrated that not only does the closely packed nanostructure enhance cell attraction effectively but amorphization selectively targets the HeLa cancer cells by embolization and/or nanoparticle internalization (e.g., endocytosis) through which the apoptosis pathway is induced. In contrast and in accordance with the SEM and FM results, the fibroblast cell line showed an enhancement of proliferation on both Cry- and Amo-SNWs. The cell attraction characteristics of Cry-SNWs and Amo-SNWs could be explained in terms of topography and surface chemistry, both of which influence protein absorption and ultimately, cell adhesion. It is hypothesized that the higher efficiency of plated-SNWs in mediating cellular attraction and induction of the apoptosis pathway compared to the free-standing SNWs is due to the immense adherent surface area provided by 3-D nanostructures as well as protein adsorption, ultimately preventing the immobilized cell from uptake followed by blocking the cell wastage that causes cell death. In addition, due to the metastatic potential of HeLa cells, viability is more likely to be hampered by immobilization. The other mechanism in which HeLa cells underwent the apoptosis pathway appears to be a result of nanoparticle internalization through cellular uptake. However, further investigations are ongoing to comprehensively understand the mechanism of nanoparticle uptake and intercellular fate. Fibroblast cells were attracted to the SNWs; however, unlike HeLa cells, they did not undergo cell death. Amorphous nanoparticles on the order of $9 \mathrm{~nm}$ caused the greatest nucleolar damage through cell membrane penetration. However, crystalline nanoparticles of nearly the same order of particle size did not induce the apoptotic pathway in the HeLa cell line. These findings suggest that amorphous SNPs in the range of 6 to $19 \mathrm{~nm}$ in diameter cause damage to HeLa cells through endocytosis. In this study particularly, we have shown the potential of silica amorphization to selectively target HeLa cells without the use of biolabels or markers and with minimal to no effect upon mammalian fibroblast cells. Moreover, in accordance with the current literature, the packing index of nanostructures is an influential factor for cellular attraction. This attribute of the platedSNW can thus be exploited to enhance the efficiency of cell-nanostructure interaction. Although fibroblast cells were not the main focus of this study, the impact of SNWs on their proliferation could be used for tissue generation and wound healing. Quantitative analysis revealed that as a result of HeLa cell interaction with high, medium and low packed Amo-SNWs, 71\%, 35.8\% and $16.8 \%$ of cells, respectively, underwent apoptosis compared to the control sample. Cry- 
SNWs, in contrast, caused cell death in $5.1 \%, 5 \%$ and $3.8 \%$ of cells. The difference in cell death is associated with the size of the nanoparticles as constrictive components of synthesized SNW. However, fibroblast cells interaction with Amo and Cry exhibited average 80 cell viability. Also the EDX result as a supplementary method to the statistical and cell morphological analysis revealed the inertness of Amo-SNWs to the fibroblast cells. However, the mechanism by which Amo-SNWs induces apoptosis-like pathway to the HeLa cells could be further investigated. Our findings also revealed that the packing index of the synthesized SNWs can be tailored by precise control of the laser repetition rate, increasing the laser pulse repetition rate from 4 to 26 $\mathrm{MHz}$, thereby causing a decrease in the nanoparticle size and an increase in the yield rate of deposition. Therefore, the closely packed nanostructures were synthesized at a higher pulse repetition rate. Moreover, the crystallinity of SNWs was found to be influenced by laser pulse width, with a prolonged pulse width capable of creating amorphous crystallinity. While additional studies are needed to fully understand the mechanisms of cellular uptake and intracellular fate of nanoparticles for different cell lines, the proposed polymorphic silica nanostructures could open an alternative avenue for developing HeLa cancer cell therapy. 


\section{CHAPTER 5}

\section{MULTIPLEX PHOTOLUMINESCENT SILICON NANO-PROBE FOR DIAGNOSTIC BIOIMAGING AND INTRACELLULAR ANALYSIS}

Keshavarz, M.; Tan, B.; Venkatakrishnan, K. “Multiplex Photoluminescent Silicon Nano-Probe for Diagnostic Bioimaging and Intracellular Analysis."Submitted to Advanced Science.

\subsection{Abstract}

We introduce herein a label-free multiplex photoluminescent silicon nano-probe (PLSN-probe) as a potential substitute for quantum dots (QDs) in bioimaging. An inherently non-photoluminescent silicon substrate was altered to create the PLSN-probe, which overcomes the major drawbacks of presently available QDs, including cytotoxicity, delivery issues and particle size dependency. Additionally, crystallinity alterations of the multiplane crystalline PLSN-probes, synthesized via an ultra-short pulsed laser (USPL) operating in the femtosecond regime, led to broad absorption and multiplex fluorescence emissions, which are attributed to the simultaneous existence of multiple crystal planes. The PLSN-probe not only demonstrates unique optical properties that can be exploited for bioimaging but also exhibits cell-selective uptake that allows the differentiation and diagnosis of HeLa and fibroblast cells. To the best of our knowledge, a label-free multiplex PLSN-probe tailored for the bioimaging of different cellular organs, with the potential ability to diagnose cancerous HeLa cells and to assess the health of a cell, has not yet been reported. Multiplex emissions of the PLSN-probe illuminate different organs of a cell such as the nucleus, nucleolemma, and cytoskeleton, depending on size-based preferential uptake by the cell organs. The PLSN-probe showed excellent biocompatibility and an affinity for being self-internalized by cancerous HeLa and mammalian fibroblast cell lines. This in vivo study revealed that cancerous HeLa cells have a higher propensity for taking up the PLSN-probe compared to fibroblast cells, allowing the diagnosis of cancerous HeLa cells. Additionally, the fluorescence intensity per unit area of the cell was found to be a reliable means for distinguishing between dead and healthy cells. Furthermore, the PLSN-probes demonstrated an enhanced lifetime and brightness when used in 
combination with either of two commonly used organic dyes: DAPI and FITC. We anticipate that the multifunctionality of the PLSN-probes will lead to better insight into the use of such probes for bioimaging and diagnosis applications.

\subsection{Introduction}

Fluorescence (FL) microscopy is extensively used as a crucial tool for biological applications such as biomedical imaging and clinical diagnostics ${ }^{83}$.(1-2) However, conventional cell imaging techniques that utilize organic fluorophores have some major problems due to their non-specific accumulation within cells and photobleaching of the dyes ${ }^{84}$. Commercially available organic dyes have been routinely used in life sciences for FL imaging; however, these dyes have some inadequacies that hinder them in the rapidly growing field of bioimaging. The main drawbacks include a limited lifetime (a few ns), progressive reduction of FL (photobleaching), low contrast in some applications, emission spectra with a red tail and low emission times. Hence, an imaging method that is not reliant on fluorophore dyes is needed ${ }^{85}$. Therefore, extensive efforts have been made to produce an alternative to organic fluorescent molecules to alleviate these problems ${ }^{86}$. In particular, recent research has highlighted the feasibility of using quantum dots (QDs) as optically efficient alternatives to fluorescent dyes for bioimaging applications. However, the applicability of QDs for live cell imaging has been severely restricted because of cytotoxicity problems, blinking, particle size dependency, and reliance on bioconjugation for delivery. Hence, methods for labeling as well as biocompatible coatings are essential for $\mathrm{QDs}^{87}$.

A wide variety of quantum-scale materials have been studied for diverse bioimaging usage; among them, semiconductor quantum dots (silicon-based QDs), noble nanoparticles (gold), superparamagnetic oxides (iron oxide), and carbon materials (SWCNTs) have been extensively exploited for optical bioimaging purposes ${ }^{88}$. As strong contrast agents, these nanoscale materials possess a range of advantages over conventional organic-based fluorescent dyes, including a higher stability in terms of photobleaching and broader excitation and narrower emittance wavelengths. For instance, cadmium selenide (CdSe) QDs have been widely used due to their broad range of emitted colors, which are tunable based on their size ${ }^{89}$. However, the cytotoxicity of QDs doped with heavy metallic constituents such as cadmium remains a serious concern ${ }^{90}$. To address this issue, a surface post-modification process is required to obtain biocompatible doped 
QDs. Despite surface modifications of the QDs, the inherent cytotoxicity of doped QDs has hampered their clinical application ${ }^{89}$. Although numerous studies have been performed to coat the toxic cores of QDs containing II-VI metallic groups, safety concerns have not yet been completely overcome, and the inherent cytotoxicity of noble QDs is one of the major obstacles for their further clinical application ${ }^{91}$.

The surface chemistry of Si-based QDs (Si-QDs) is of great interest because of their considerable difference from heavy metal QDs. In addition, the utility of Si-QDs in bioimaging lies in their optical properties, which are size- and crystallinity-dependent. Si-QDs have shown a substantial breakthrough as a bioimaging agent due to their nontoxicity and biodegradability; one byproduct of degradable Si-QDs, silicic acid, can be readily excreted via the urine. Although Si-QDs have drawn attention due to their superior biocompatibility, key challenges of high quantum yield and long-term stability in water and biological media must be overcome ${ }^{92}$. Additionally, the delivery of Si-QDs is dependent on bioconjugation ${ }^{93}$. Numerous studies have been reported on the targeted usage of bioimaging agents. For instance, Tilley et al. demonstrated non-specific uptake of allylamine-terminated blue-emitting Si-QDs in HeLa cells ${ }^{94}$. Reipa's laboratory conjugated redemitting silicon nanoparticles to streptavidin and demonstrated specific binding of the nanoparticles to biotinylated polystyrene beads ${ }^{95}$. Despite progress in bioconjugation chemistry, the size-tunable luminescence characteristics, as well as the minimal auto-fluorescence of the $\mathrm{Si}$ QDs, present challenges ${ }^{96}$. One of the customary coatings of QDs, mercaptoacetic acid, has been found to be cytotoxic ${ }^{97-98}$. The exposed metallic core of coated QDs due to dissolution of the coating can also be toxic ${ }^{97}$. Cadmium and selenium ions, which are used in the core of QDs, are known to be cytotoxic ${ }^{98}$. In addition, erosion of the shell may cause undesirable reactions in vivo ${ }^{97}$. QDs may be toxic based on their composition, as reported in in vitro studies ${ }^{97}$. CdSe QDs coated with mercaptoacetic acid were found to be toxic to rat pheochromocytoma cells ${ }^{97}$. The mechanism of cell death is unknown, but it is believed to be caused by the free $\mathrm{Cd}$ released by core degradation ${ }^{99}$.

Moreover, QDs are highly sensitive to surface defects, which can reduce the quantum yield of QDs by affecting the recombination of electrons and holes, resulting in blinking of the QDs ${ }^{100}$. This flaw, however, can be diminished by coating a shell around the QD ${ }^{100-101}$. QDs have shown a strong tendency to aggregate when placed in live cells, which interferes with cell function ${ }^{102}$. A difficulty also exists in delivering QDs inside cells without killing and/or damaging the cells in the 
process $^{103}$. Hence, conjugation of the QDs with different biomolecules or bio-labels is required ${ }^{104}$. Although QDs are in the nanometer size range, bioconjugation increases their size, rendering their delivery into cells more difficult ${ }^{105}$.

Here, we report the first development of multiplex emissions from PLSN-probes. The transition from a single (polyhedron) to multiplanar (polyhedral) silicon crystal structure yields a higher absorption and hence a superior FL throughput. Unlike QDs, the PLSN-probes not only exhibit superior optical properties including higher brightness and narrower emission and broader absorption spectra, but also cell-selective uptake, which eliminates the need for coating and bioconjugation. This unique nano-probe emits a higher FL intensity when there is a greater number of crystal planes (polyhedral PLSN-probes), and it is hypothesized that the multi-crystal orientation is the cause of the multiplex emission of the PLSN-probes, which has never been observed with QDs.

The use of ultra-short pulsed laser (USPL)-assisted ionization to synthesize PLSN-probes under inert atmospheric conditions resulted in impurity-free and well-characterized 3D structures. The PLSN-probes were created based on kinetic self-assembly activated by multiphoton ionization. By using USPL, we were able to systematically transform a non-luminescent bulk single-crystal silicon wafer into multiplex photoluminescent nano-probes with tunability via synthesized crystal orientations $^{106}$. The higher number of concurrent crystal planes increases the absorption and subsequently the FL emission of the PLSN-probes. The wide-range absorption and multiplex narrow emittance of the PLSN-probes, wavelength-dependent phenomena, were exploited to illuminate different organs of the cells. Internalization of the PLSN-probes into cells allowed intercellular observation at the distinct excitation wavelength, demonstrating that the functions of multiple dyes can be simultaneously mimicked by PLSN-probes. The size distribution of the synthesized PLSN-probes was regulated to dimensions in which the quantum confinement effect is preeminent by precisely tuning the laser fluence. The smaller-sized Si-NPs, ranging between 2 and $2.6 \mathrm{~nm}$, show an affinity for internalization, allowing the cell nucleus and nucleolemma to be discerned. PLSN-probes measuring $3.5 \mathrm{~nm}$ were also permeable through the cell membrane and were able to indicate details of external organs such as the cytoskeleton, microtubules, and lamellipodium. Additionally, this versatile method of bioimaging has shown diagnostic competency through which HeLa cancer cells can be differentiated from mammalian fibroblast cells. Moreover, dead and viable cells can be screened by a comparison of emitted intensities. The 
PLSN-probes showed excellent biocompatibility and high photostability within live cells for longer periods compared to fluorescent dye alone. The biocompatibility and photostability of the PLSN-probes were examined after 24 and 48 hours of incubation on two HeLa and fibroblast cell lines. Notably, this is the first report on the use of PLSN-probes as a cell-selective uptake bioimaging agent with the direct possibility of diagnosing HeLa cells as well as a general efficiency for bioimaging.

\subsection{Materials and methods}

To investigate the morphology and dimensions of the synthesized PLSN-probes, a JEOL JSM4800 field emission scanning electron microscope (FE-SEM) and a JEOL JEM 2100 highresolution transmission electron microscope (HR-TEM) were used. HR-TEM was performed to determine the size distribution and morphology of the PLSN-probes; for this method, a droplet of suspended PLSN-probes was placed on a copper grid for direct observation after drying. Image analysis software was used to analyze the HR-TEM micrographs, perform size measurements and compile a distribution histogram. An HR-TEM diffraction pattern (FFT) was also obtained to confirm the formation of crystalline PLSN-probes. Energy dispersive X-ray spectra (EDX) analyses using Oxford Instruments further corroborated the formation of pure PLSN-probes. The Raman spectra were recorded with a Bruker SENTERRA dispersive Raman microscope using a wavelength of $325 \mathrm{~nm}$. The Raman spectra showed a strong band and sharp peaks at $521 \mathrm{~cm}^{-1}$, which is characteristic of crystalline PLSN-probes. Ultraviolet-visible-near-infrared (UV-VisNIR) absorption and reflection spectra were obtained with a Hitachi UV-3100 UV-Vis-NIR spectrophotometer. Photoluminescence (PL) spectra were recorded on a Shimadzu RF-5301PC spectrofluorophotometer at room temperature.

\subsubsection{Synthesis of PLSN-probes}

A single-crystal N-type silicon wafer with a (100) crystallographic was sliced into 2 - $\mathrm{cm}^{2}$ squareshaped samples, which were ultrasonically cleaned in a $50^{\circ} \mathrm{C}$ acetone bath for 15 minutes, followed by rinsing and drying. To synthesize the PLSN-probes, silicon substrates were exposed to a diode- 
pumped Yb-doped femtosecond laser beam (Clark-MXR, Inc., IMPULSE Series ultra-short pulse laser). This laser system is capable of producing central wavelengths of $1040 \mathrm{~nm}$ with a pulse width that can be varied, ranging from $214 \mathrm{fs}$ to $1428 \mathrm{fs}$, as well as a tunable laser pulse repetition rate that can vary between $4 \mathrm{MHz}$ (low rep. rate) and $26 \mathrm{MHz}$ (high rep. rate). A maximum average operating power of $16 \mathrm{~W}$ was attainable. Utilizing the ultra-short (femtosecond range) pulsed laser (USPL) is advantageous since the absorption of multiphoton excitation energy delivers the required energy of ionization and also, given the localized laser beam spot within the focal point, results in precise control over the synthesized PLSN-probes. The samples were, therefore, irradiated at the beam focal point, with a computerized galvano scanner precisely implementing the synthesis processes. The self-assembled PLSN-probes were synthesized by single-step femtosecond laser processing of the silicon substrate. For the purpose of this study, we fabricated the polyhedron, intermediate and polyhedral PLSN-probes with a pulse width of $214 \mathrm{fs}$ and repetition rates of 4, 12 and $26 \mathrm{MHz}$, respectively. The laser fluences at 4, 12 and $26 \mathrm{MHz}$ were measured as $1.57,3.39$ and $10.18 \mathrm{~J} / \mathrm{cm}^{2}$, respectively. (The laser power was $16 \mathrm{~W}$, with a Gaussian $1 / \mathrm{e}^{2}$ beam diameter of $10 \mu \mathrm{m}$, and the scanning speed was $2 \mathrm{~mm} / \mathrm{s}$; all parameters were held constant.)

\subsubsection{Cell culture and seeding}

The synthesized PLSN-probes were sterilized under UV light for 20 minutes prior to their addition to cell culture medium. Fibroblasts (NIH3T3) and human cervical cancer cells (HeLa, ATCC, American Type Culture Collection, ATCC No. CCL-2) were employed in cell experiments to ascertain the comparative functionality of mammalian and cancer cell lines in response to the samples. Fibroblast cells were grown in DMEM containing 10\% heat-activated fetal bovine serum with $1 \%$ penicillin-streptomycin antibiotics (Pen-strep). HeLa cells were grown in DMEM-F12 supplemented with $10 \%$ fetal bovine serum and 1\% Pen-strep. Subsequently, the cells were separately cultured on substrates placed in Petri dishes with a seeding density of $750,000 \mathrm{cells} / \mathrm{cm}^{2}$. The Petri dishes were placed in an incubator for 24 and 48 hours at $37{ }^{\circ} \mathrm{C}$. Following the PLSNprobe cell response study, the cells were studied via SEM and confocal FL microscopy. 


\subsubsection{Cell imaging}

The morphology of the cells was observed using a scanning electron microscope (SEM) (Hitachi, SU1510). For this aim, after the prescribed time period, the spent medium was aspirated. The samples were then fixed in $2 \%$ glutaraldehyde in $0.1 \mathrm{M} \mathrm{pH} 7.3$ sodium cacodylate buffer for 1 hour. Next, the samples were dehydrated at increasing concentrations of alcohol for 20 minutes, followed by immersion in $0.1 \mathrm{M}$ sodium cacodylate buffer with $0.2 \mathrm{M}$ pH 7.3 sucrose for 20 minutes. The samples were then critical point dried. After the experiment, the cells were prepared for direct observations via SEM.

\subsubsection{Fluorescence microscopy}

For FL microscopy, the samples were first fixed in methanol-free paraformaldehyde followed by incubation in skim milk to prevent non-specific binding. To stain the actin and cytoskeleton, the samples were incubated with Alexa Fluor 488 (Life Technologies) followed by DAPI (Life Technologies) to stain the nucleus. The samples were studied using a FL microscope (Nikon, Canada). Seeded cells were also fluorescence-imaged on a Zeiss LSM 710 META upright confocal laser-scanning microscope using 60× magnification water-dipping lenses for the monolayer cultures. Wavelengths of 405, 488, 555 and $633 \mathrm{~nm}$ were used. Image data acquisition and processing were performed using a Zeiss LSM Image Browser, Zeiss LSM Image Expert and ImageJ. Statistics; All experiments were performed in triplicate, and the data points were averaged unless otherwise indicated. The error bars show standard deviations.

\subsection{Results and discussion}

\subsubsection{Fabrication and structural characterization}

The PLSN-probes were synthesized via USPL-assisted ionization. The absorption of one or more photons by electrons in atoms of the substrate (a single-crystal silicon wafer) causes spatial electron separation of the excited atom or ion species. To prevent any compositional changes, this 
process was carried out in the presence of an inert gas. The USPL has shown a capability of overcoming the ionization threshold of the silicon substrate by inducing sufficient photon energy. The accumulated energy of multiphoton absorption exceeds the ionization potential of the silicon and hence leads to atomic ionization. Therefore, the kinetics of the self-assembly process were initiated by multi-photoionization. The experiment showed that multi-photoionization can be governed by precisely tuning the laser fluence. When a sufficiently higher laser fluence interacts with the substrate, it results in the formation of a 3D self-assembled structure consisting of multiplanar crystal Si (polyhedral). Likewise, a lower laser fluence resulted in fewer crystal planes (polyhedron). The synthesis of the PLSN-probes can be precisely tuned by regulating the laser parameters.

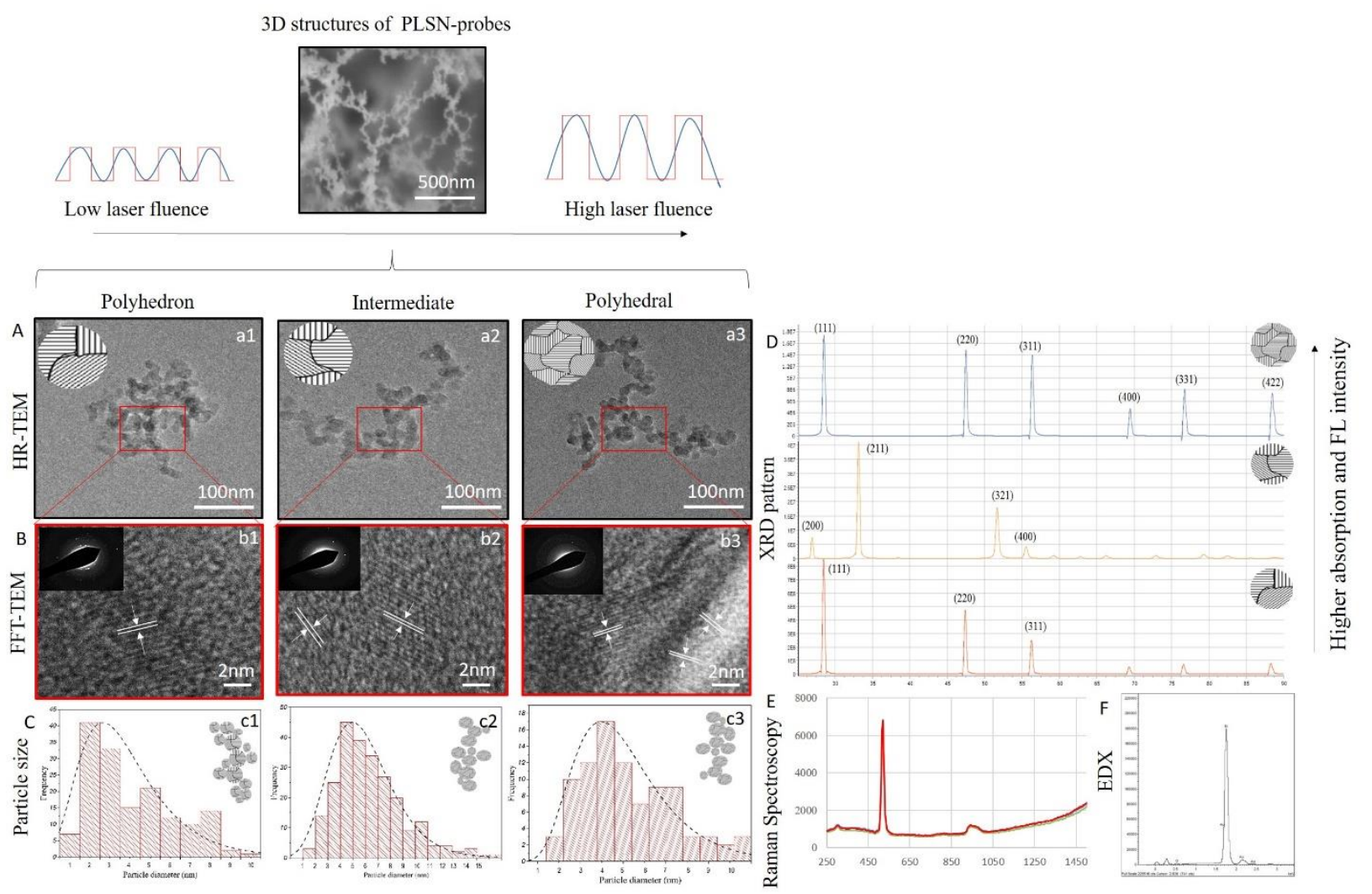

Figure 5.1: Characterization of the PLSN-probes. HR-TEM micrographs illustrating (a1) polyhedron, (a2) intermediate and (a3) polyhedral PLSN-probes. Crystal d-spacing and diffraction are demonstrated in images (b1-b3). (C) Size distribution of the fused PLSN-probes plotted in histograms (statistical analysis performed over 1000 random particles with an SD of 0.7). XRD patterns (D) show the dominance of multiple crystal planes upon increased laser fluence. Raman spectroscopy (E) demonstrates the formation of crystalline PLSN-probes. EDX results (F) acquired from the synthesized PLSN-probes do not indicate any compositional changes. 
While the laser pulse duration and laser power were kept constant at $214 \mathrm{fs}$ and $16 \mathrm{~W}$, respectively, high, intermediate and low laser fluences were achieved at a 4-, 12- and 26-MHz repetition rate, respectively. The synthesized PLSN-probes form a deposition on the substrate, which was ultrasonicated prior to characterization and cell culture.

Transmission electron microscopy (TEM) analysis was carried out to study the crystallinity and size distribution of the synthesized PLSN-probes. HR-TEM micrographs (Figure 5.1 (A)) show fused sub-nano spherical silicon particles with a narrow size distribution in the range of 2-10 \pm 0.7 $\mathrm{nm}$ (Figure 5.1 (C)). The presence of multiple crystal orientations in the polyhedron, intermediate and polyhedral PLSN-probes was also observed on FFT-HR-TEM micrographs (Figure 5.1 (B)). The alteration of the crystal planes, along with the maintained size distribution range, rules out the influence of size range on the further optical characteristics of the as-synthesized PLSN-probes.
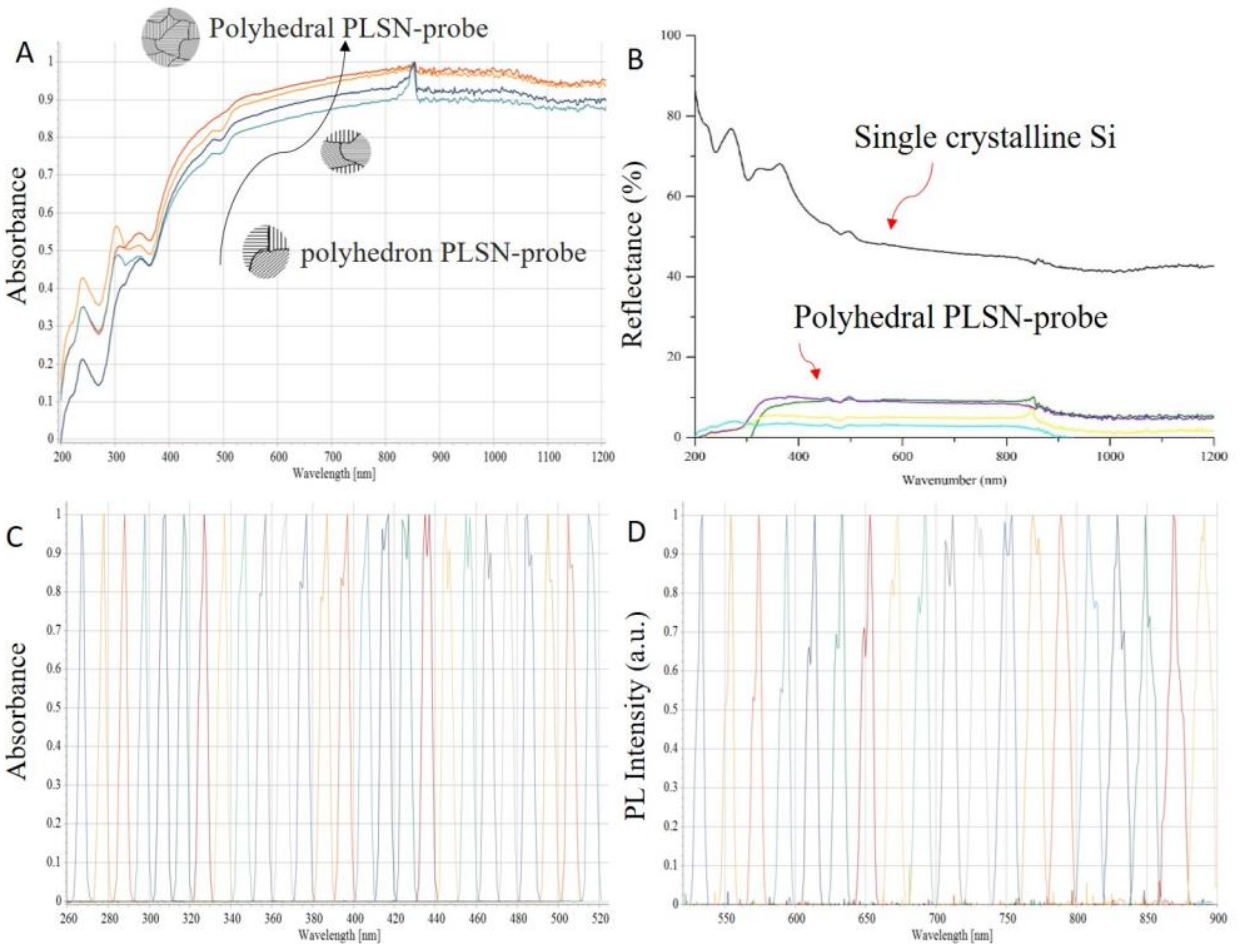

Figure 5.2: Optical properties of the PLSN-probes with respect to crystal plane variation. (A) UV-Vis-NIR spectra of the PLSNprobes exhibit an increase in photon absorption with polyhedral PLSN-probes in the Vis and NIR regions. As the number of crystal planes increases, the absorption increases. (B) Reflectance spectra of the PLSN-probes. The black color spectrum corresponds to the silicon substrate (reference). The PLSN-probes at different ranges of particle size show relatively the same reflection/absorption. The reflectance over the entire UV and NIR band is lowered. PLSN-probes show significantly higher optical absorption, especially in the UV and NIR regions. (C-D) Room-temperature photoluminescence (PL) spectra of the polyhedral PLSN-probes show narrow emission with a significantly large Stokes shift. 
Additionally, X-ray diffraction (XRD) analysis (Figure 5.1 (D)) further confirms the dominance of 3, 4 and 6 multiple crystal planes upon increased laser fluence. Chemical and elemental analyses of the PLSN-probes were carried out by EDX (Figure 5.1 (F)) and Raman spectroscopy (Figure 5.1 (E)), where no trace of chemical changes was detected. EDX confirmed the presence of pure silicon, and the Raman spectrum proved the crystallinity of all the synthesized PLSN-probes as well. All of the evidences demonstrate the successful formation of pure 3D structured PLSNprobes with varied crystal planes at the same size distribution range.

\subsubsection{Optical characterization}

The PLSN-probes were first investigated in terms of UV-Vis-NIR reflection and absorption at room temperature, as shown in Figure 5.2 (A-B). Compared to the bulk silicon, irradiation of the PLSN-probes ranging from 200 to $2200 \mathrm{~nm}$ exhibited a significantly lower reflection, which can be translated to high photon absorption, as shown in Figure 5.2 (B). However, higher absorption was observed for the polyhedral PLSN-probes. The transformation of single-crystal silicon (substrate) to polycrystalline PLSN-probes at different ionization levels resulted in an alteration of the dominant crystal planes and, ultimately, an enhancement in absorption properties (Figure 5.2 (A)). The UV-Vis-NIR spectroscopy revealed that strong absorption occurs in the polyhedral PLSN-probes. This higher absorption is attributed to the distinct photon absorption of each available crystal plane. In particular, lower absorption was observed at a lower wavelength of the UV region, whereas upon irradiation of Vis light ( 400-700 nm), the synthesized PLSN-probes showed higher absorption and stunted reflection. Figure 5.2 (C) presents the photoluminescence (PL) spectra of the polyhedral PLSN-probes at room temperature. All of the samples showed strong PL with respect to the excitation wavelength. The emission at a longer wavelength and hence lower intensity demonstrate the FL excitation of the PLSN-probes. Notably, broad absorption and relatively narrow emission with a 300-nm Stokes shift were observed. As the excitation wavelength increased, the emission shifted toward a longer wavelength. However, the FL intensity decreased at wavelengths longer than $700 \mathrm{~nm}$. The overall narrow FWHM and long Stokes shifts are the two major PL characteristics that are significant for successful application of the PLSN-probes for bioimaging ${ }^{107}$. Since the PLSN-probe consists of fused particles ranging from 2 to $10 \mathrm{~nm}$, broader absorption and a subsequent wide range of emission were achieved. The FL 
intensity showed an $80 \%$ increase as the excitation wavelength shifted toward $600 \mathrm{~nm}$, after which the FL intensity decreased and the emission color was gradually redshifted. Moreover, irradiation of the PLSN-probes in the visible range, shown in Figure 5.3 (A), demonstrated higher absorption of the polyhedral PLSN-probes compared to the polyhedron PLSN-probes. However, both probe types exhibited multiplex emissions at excitation wavelengths ranging from 405 to $633 \mathrm{~nm}$, as shown in Figure 5.3 (B). A plot of the normalized FL intensity (Figure 5.3 (C)) reveals the higher emission intensity of the polyhedral PLSN-probes. Hence, the polyhedral PLSN-probes were chosen for further bioimaging studies. Strong changes in FL emission is not caused by conjugated lengths, making the PLSN-probes suitable for bio-applications in which the toxicity of fluorescent agents and their lengths are primary concerns.

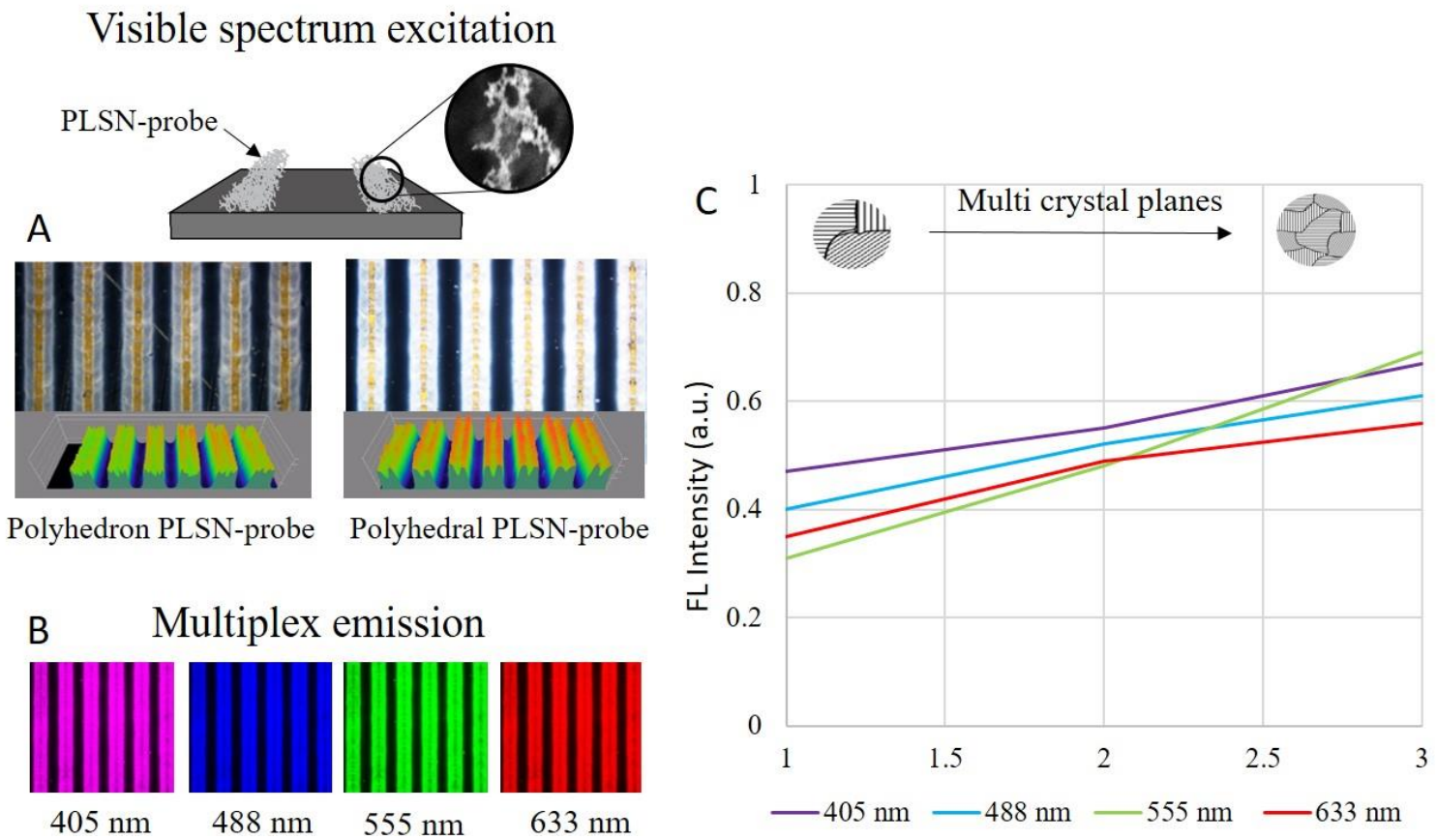

Figure 5.3: Influence of the multi-crystal planes on PLSN-probes. Irradiation at the visible spectrum (A) shows a stronger excitation of the polyhedral PLSN-probes. Emission of the polyhedral PLSN-probes (B) at excitation wavelengths of 405, 488,555 and $633 \mathrm{~nm}$ exhibits the multiplex characteristics of the PLSN-probes. Changes in normalized FL intensity due to crystallinity alteration of the PLSN-probes are shown in (C). A comparison of florescence intensity at excitation wavelengths of 405, 488, 555, and $633 \mathrm{~nm}$ shows the higher emission intensity of the polyhedral PLSN-probes.

The results imply that by varying the excitation wavelength, different FL emissions ranging from 500 to $800 \mathrm{~nm}$ can be achieved. Furthermore, for excitation at a longer wavelength, a redshift 
emission at $650 \mathrm{~nm}$ can be interpreted from the excitation and emission profile. Since the FL domain of the PLSN-probes is wavelength-dependent, the presence of a diverse size distribution was neutralized by narrowing the excitation wavelength. This phenomenon also revealed that size variations in the PLSN-probes may allow one to precisely target different organs of the cell, which can then be distinguished at distinct excitation wavelengths. Figure 5.3 (C) depicts a higher FL peak intensity observed with polyhedral PLSN-probes at four excitation wavelengths (405, 488, 555 and $633 \mathrm{~nm}$ ) commonly used in bioimaging.

The acquired result from excitation and emission spectra fits well with observations from confocal FL microscopy. The pronounced variational excitation features evince that the PLSN-probes are indeed multiplex emissive and wavelength-dependent. These attributes may be useful in exploring the implementation of polyhedral PLSN-probes for bioimaging.
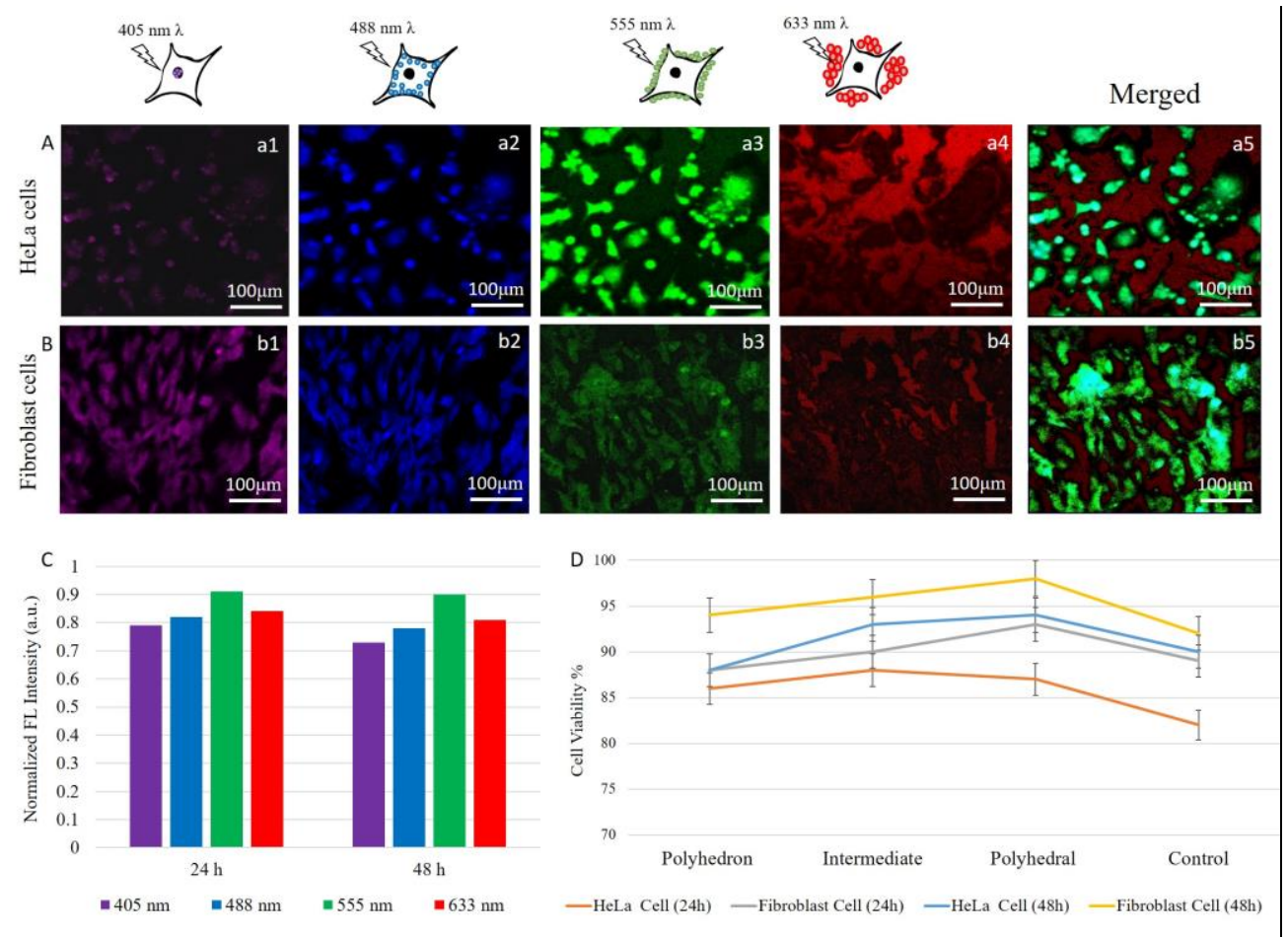

Figure 5.4: Cell-selective uptake of the PLSN-probes. Confocal microscopy images of HeLa (A) and fibroblast (B) cell lines without any fluorophores. Image of the cells after incubation with PLSN-probes at 405, 488, 555 and 633 nm revealed cell-selective uptake. HeLa cells exhibited sequential uptake (a1-a4) through which different layers of the cell, including the cytoskeleton, nucleolemma and nucleus as well as the cell periphery, were screened. Images of fibroblast cells (b1-b4) imply an aversion toward cellular uptake as only the cytoskeleton of the cells could be discerned. Merged images taken at different excitation wavelengths display intercellular details of HeLa cells (a5) but only the cell periphery and cytoskeleton of the fibroblast cells (b5). The FL intensity of the multiplex PLSN-probes after 24 and 48 hours of incubation (C) revealed negligible intensity decay. A cell viability assay (D) demonstrated superior biocompatibility of the PLSN-probes after 48 hours on both HeLa and fibroblast cells. 


\subsubsection{Bioimaging and intercellular analysis}

We investigated the possible use of PLSN-probes for optical imaging of cancerous HeLa and mammalian fibroblast cells. Confocal FL microscopy of the HeLa and fibroblast cells at four excitation wavelengths, 405, 488, 555 and $633 \mathrm{~nm}$ (Figure 5.4 (A, B)), revealed the multiplex attribution of the PLSN-probes, by which the probes can be excited at different wavelengths. This characteristic was exploited to illuminate different parts of the cell, each at a distinct wavelength. The in vitro uptake of the PLSN-probes by living cells was studied to identify the location of the internalized PLSN-probes within the cells. Strikingly, HeLa cells exhibited selective uptake, as shown in Figure 5.4 (a1-a4). As the PLSN-probe internalizes through the cell membrane, it breaks apart within the cell body and further penetrates into the different layers of the cell. The purple emission at the 405-nm excitation wavelength of the PLSN-probes was primarily observed from the nuclear region while the blue and green emissions, excited at 488 and $555 \mathrm{~nm}$, correspond to the nucleolemma and cytoskeleton of the cell. The red emission displayed PLSN-probes that had not been taken up and, therefore, showed only the periphery of cells. In this case, however, this phenomenon was found to be restricted to the HeLa cells. Figure 5.4 (A) depicts the sequential uptake penetration of the PLSN-probes. Because the emission of shorter wavelengths correlated to the size of the probes, the ability to visualize the nuclei at $405 \mathrm{~nm}$ implies that smaller PLSNprobes had penetrated the cell nuclei. Likewise, the nucleolemma and cytoskeleton were similarly distinguished. Our results reveal that the PLSN-probes of approximately $2 \mathrm{~nm}$ tended to infiltrate the nuclei whereas the slightly larger PLSN-probes of approximately $2.6 \mathrm{~nm}$ surrounded the nucleolemma. Similarly, the 3.5-nm PLSN-probes were shown to be attracted to the cytoskeletonmembrane. The 6.2-nm PLSN-probes could not permeate the cell membrane, and hence, these PLSN-probes, excited at $633 \mathrm{~nm}$, displayed the periphery of the cells. However, as depicted in Figure 5.4 (b1-b4), the uptake differed in the fibroblast cells for this imaging agent, and only the cytoskeleton could be visualized at all four tested wavelengths. The cell-selective uptake is hypothesized to be due to the difference in permeability of these two cell lines, leading to the potential use of the PLSN-probes for diagnosis of HeLa cells. The limited uptake of the PLSNprobes by the fibroblast cells can be exploited to distinguish between HeLa and fibroblast cells. In addition, the biocompatibility of the PLSN-probes was assessed after 24 and 48 hours of incubation. A cell viability assay (Figure 5.4 (D)) showed the superior biocompatibility of the 
PLSN-probes. The FL intensity of the PLSN-probes was also examined over the course of 24 and 48 hours to rule out the influence of gradual disintegration; as shown in Figure 5.4 (C), negligible differences and exceptionally strong FL intensities were observed during the 48 hours of incubation.

\section{D Imaging of Cell organelles}

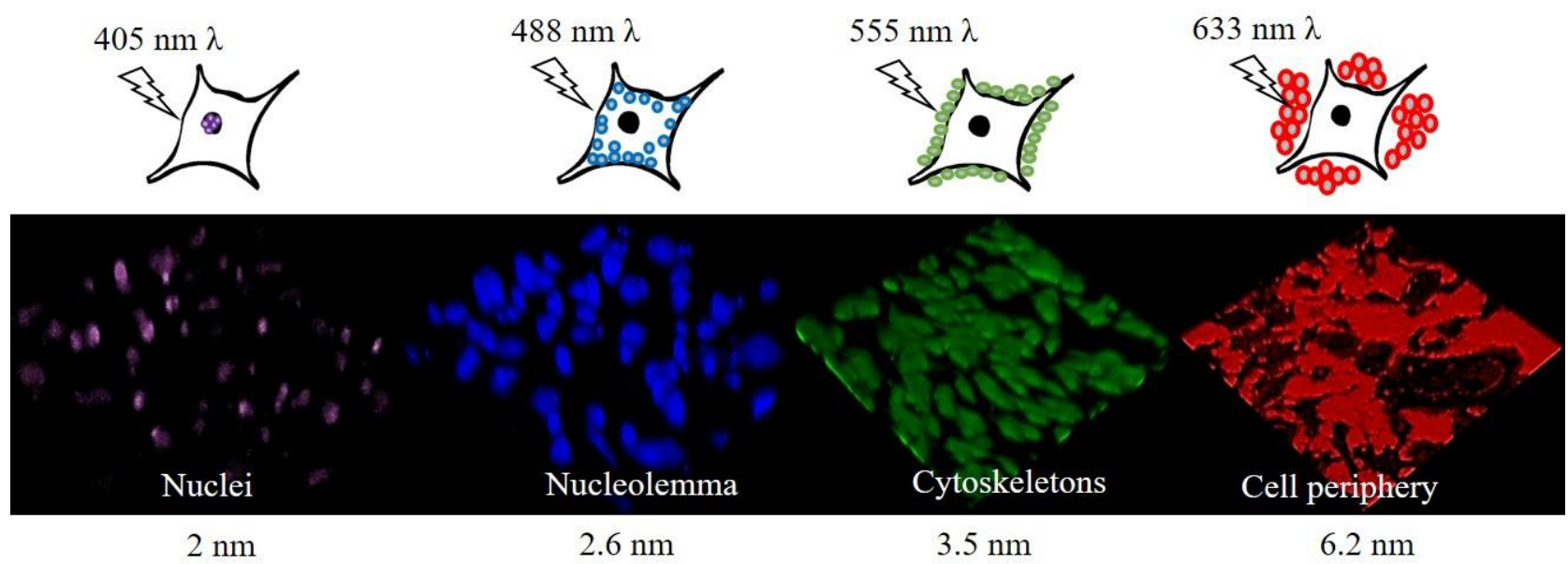

Figure 5.5: Intracellular analysis of the HeLa cells. Selective uptake of the PLSN-probes made it possible to visualize intercellular organs of individual cells. From left to right: nuclei, nucleolemma, cytoskeleton and the cell periphery were depicted by internalization of the PLSN-probes.

Figure 5.5 shows the 3D visualization of intracellular organs of HeLa cells by confocal FL microscopy. These images provide details regarding the uptake and aggregation of the PLSNprobes within a cell. To the best of our knowledge, this is the first report on the use of PLSNprobes that are cell selective and possess a multiplex characteristic for 3D imaging of live cancer cells. As demonstrated in Figure 5.4, these nano-probes did not have a tendency to aggregate, which would cause cellular damage as well as lessen visibility. The agglomeration of nanoscale particles and non-specific accumulation of organic dyes are a common drawback of these imaging agents but has not been observed with the PLSN-probes ${ }^{108}$. 

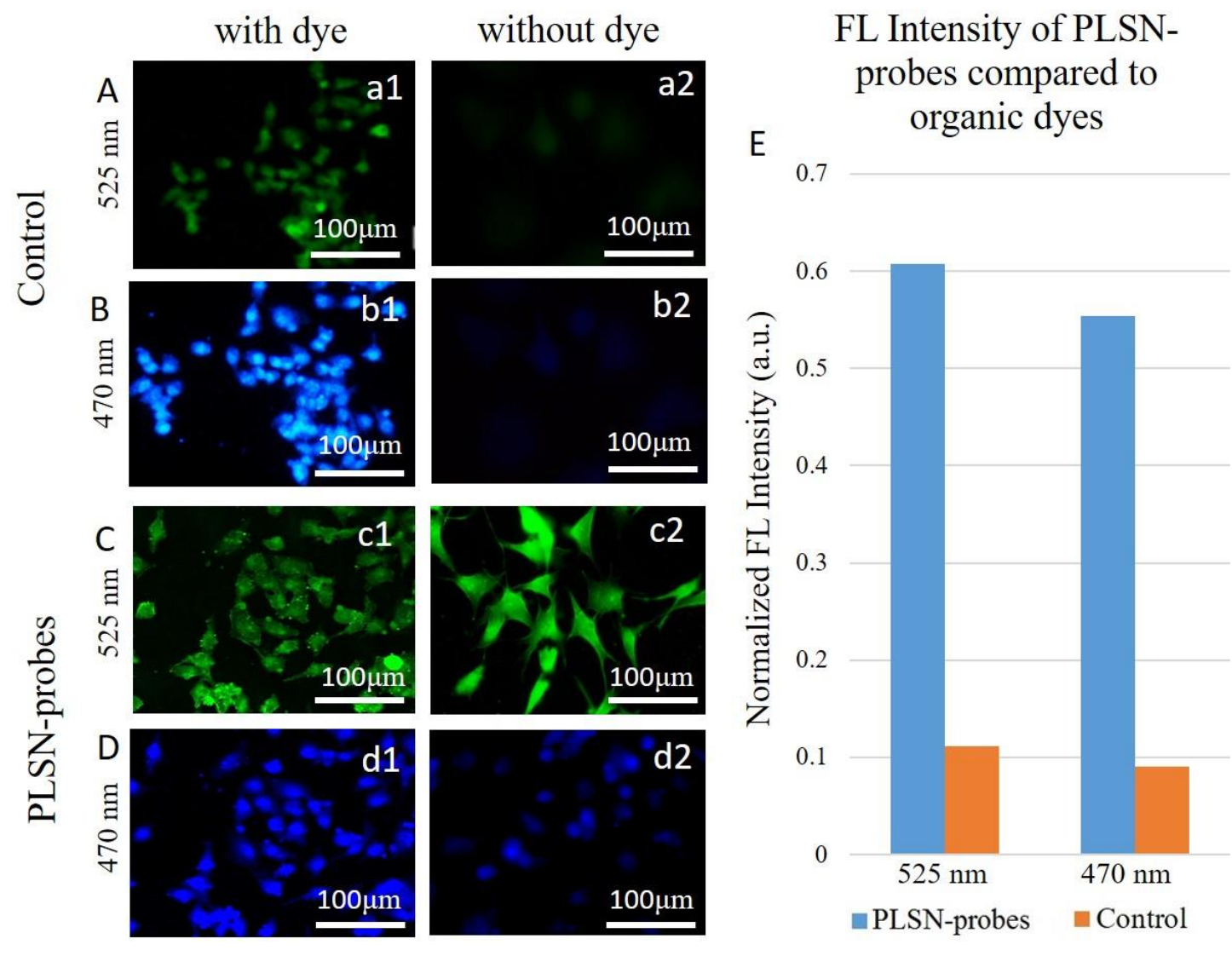

Figure 5.6: PLSN-probes for FL bioimaging. (A-B and C-D) show FL imaging of cells on the control sample and in the presence of PLSN-probes, respectively. The intensity of emitted spectra plotted in (E) illustrates the remarkable FL intensity of the PLSNprobes compared to the control without dyes.

To determine the application prospects of these novel PLSN-probes as efficient bioimaging tools, they were compared to two commonly used dyes, DAPI and FITC (control), in the absence of the PLSN-probes, as shown in Figure 5.6 (A, B). The cells were prepared for FL imaging by adding a small aliquot of the PLSN-probes (without the addition of any dyes) to aqueous cell culture medium, followed by fixation. The results are demonstrated in Figure 5.6 (c2-d2). Cells stained with DAPI and FITC in the presence of PLSN-probes displayed brighter specification (Figure 5.6 (c1-d1)) relative to the control (Figure 5.6 (a1-b1)). The higher FL intensity of the PLSN-probes compared to DAPI and FITC was measured, as shown in Figure 5.6 (E). Comparison of the FL microscopy of samples stained with dyes and those supplemented with PLSN-probes shows a significant difference in which control cells are not distinguishable (Figure 5.6 (a2-b2)). In contrast, the PLSN-probes strongly enhanced the FL imaging and emitted in the visible region 
when excited at both 470 and $525 \mathrm{~nm}$, as shown in Figure 5.6 (c2-d2). Therefore, as is evidenced in Figure 5.5, the PLSN-probes dispersed in cell culture medium can be substituted for organic dyes. Additionally, quantitative assessment of their FL intensities (Figure 5.6 (E)) implies a higher emission intensity of FITC and DAPI dyes when assisted with the PLSN-probes as compared to samples stained with only FITC and DAPI dyes.
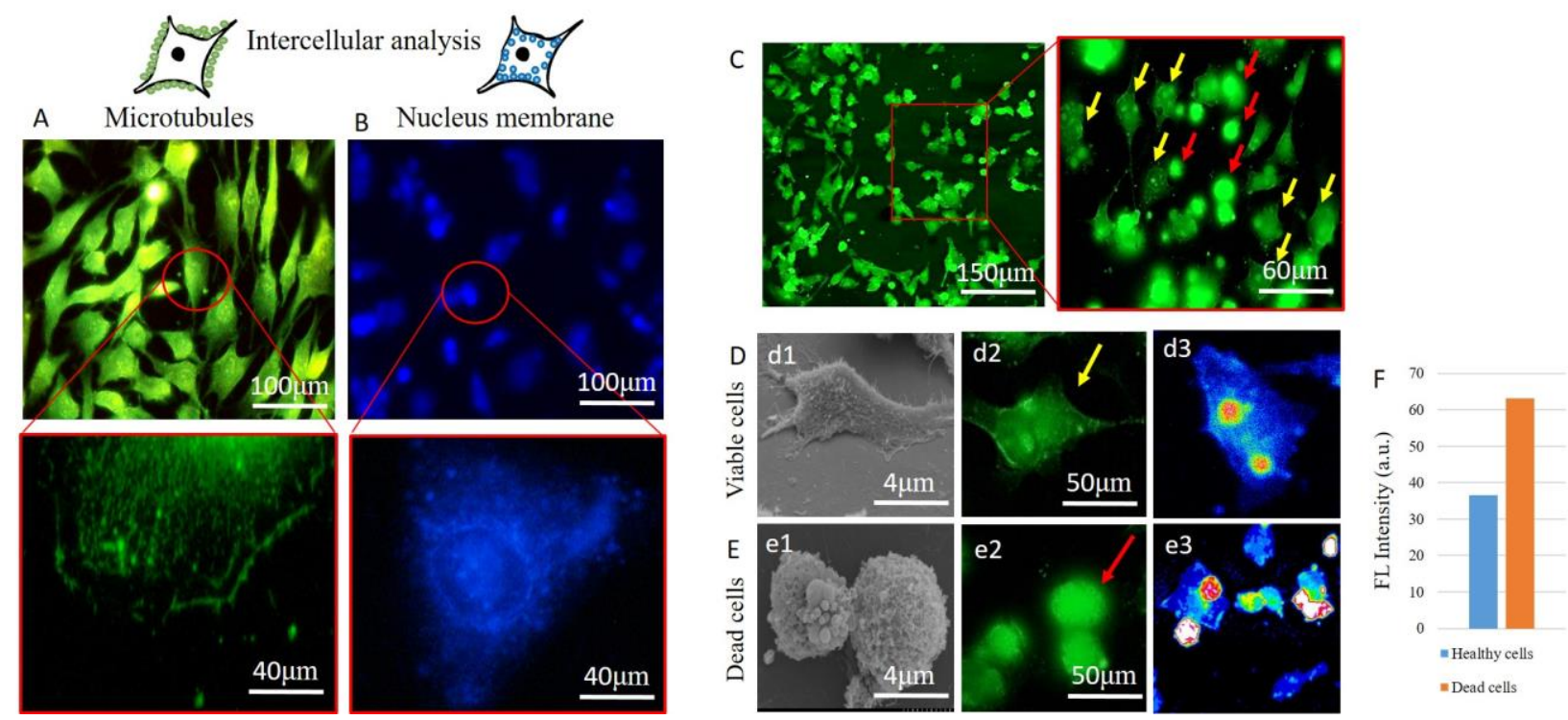

Figure 5.7: Studying the health of a cell. (A) PLSN-probes in the size range of 3-5 $\mathrm{nm}$ showed an affinity to the cytoskeleton, by which microtubules could be visualized. (B) PLSN-probes ranging from 2-3 nm diffuse through permeable cell membranes and adhere to the nucleus/nucleolemma. Diagnosis of HeLa cells. An FL image (C) shows the overall view of viable and dead cells (dead and healthy cells are indicated by red and yellow arrows, respectively, in the higher-magnification FL image on the right). SEM and FL images demonstrated viable cells (d1 d2) and dead cells (e1, e2). The intensity gradient of the FL images exhibits the dispersion of PLSN-probes within healthy (d3) and dead cells (e3). A comparison of the FL intensities per unit area is shown in (F) and indicates a significantly higher intensity from the dead cells.

The varied size distribution of the PLSN-probes not only makes them wavelength-dependent but also results in a bifunctionality by which different organs of the cell can be differentiated (Figure 5.7 (A, B)). As demonstrated in Figure 5.7 (A), the PLSN-probes internalized into the cytoskeleton allow one to visualize the microtubules. Since internalization of the PLSN-probes does not exhibit cytotoxicity or other cellular damage, it is hypothesized that uptake of the PLSN-probes occurred through the microtubules, as they are components of the cytoskeleton found throughout the 
cytoplasm. An affinity of the smaller PLSN-probes to the nucleus inside the cytoplasm was also observed, as shown in Figure 5.7 (B).

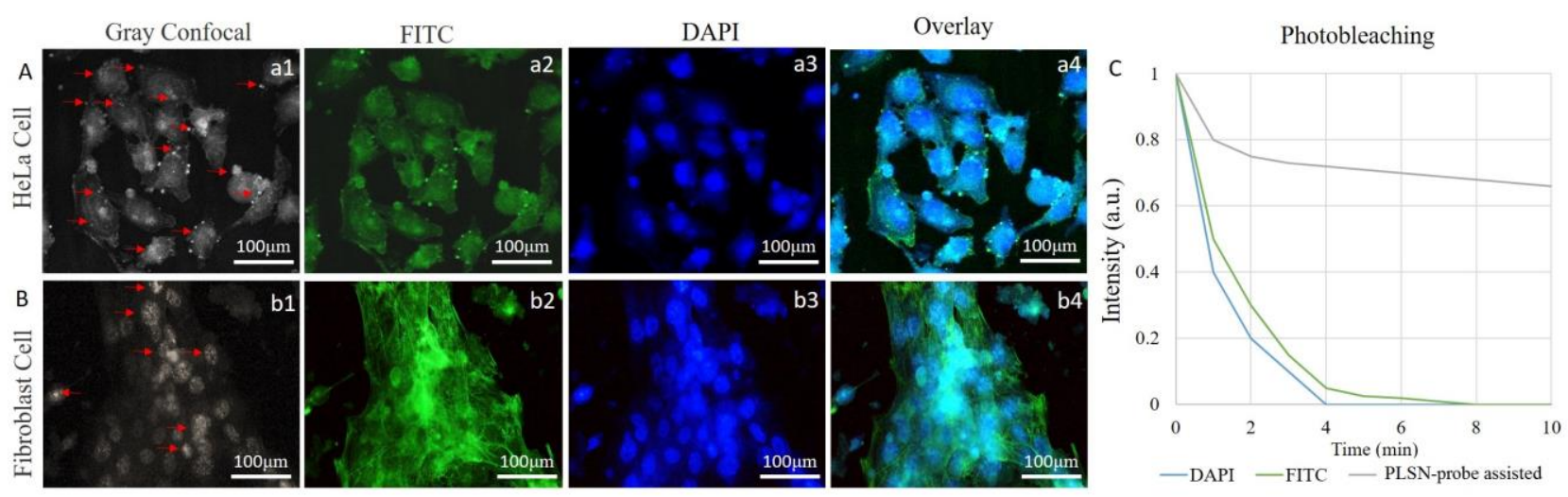

Figure 5.8: PLSN-probe-assisted FL imaging of HeLa (A) and fibroblast (B) cells. Confocal imaging of HeLa (a1) and fibroblast (b1) cells shows the uptake of the PLSN-probes, as indicated by arrow markers. Cells were stained with FITC and DAPI for both cell lines (a2-a3, b2-b3). Overlay of blue and green emissions (a4, b4). Photobleaching of FITC and DAPI is displayed in (C).

Aside from using the PLSN-probes for bioimaging purposes, the unique label-free cellular uptake attribute of these probes has demonstrated their potential use for diagnosing cell health. The diagnostic application of PLSN-probes relies on the concentration of internalized PLSN-probes in the cytoplasm, by which the intensity of the emitted light drastically increases. Cell shrinkage as a result of cell death was found to be distinguishable through the usage of PLSN-probes, as displayed in Figure 5.7 (C-E). The FL intensity per unit area of dead cells was two-fold greater than that of normal (healthy) cells. This offhand diagnostic method is an additional advantage of the synthesized PLSN-probes. The overall image (Figure 5.7 (C)) shows the presence of dead and healthy/viable cells. Normalization of the FL intensity per unit area of the cell as a measure to identify the PLSN-probe uptake provides a means to differentiate dead cells from viable cells (Figure 5.7 (D-E)). As plotted in Figure 5.7 (F), the intensity of the dead cells was almost twice that of the viable cells. This tool provides a simple method for distinguishing dead from healthy cells. Thus far, organic dyes are the only current imaging means with this function. To the best of our knowledge, a nanoscale, label-free probe with the capability of screening cellular health has not yet been reported. Of note, this characteristic was found to be dominant in the PLSN-probes excited at $555 \mathrm{~nm}$ or, in other words, the PLSN-probes ranging in size between 3-5 $\mathrm{nm}$. 
With the aim of overcoming the aforementioned flaws of organic dyes, two commonly used fluorescent stains, DAPI and FITC, were applied simultaneously with the PLSN-probes. Figure 5.8 shows results from the PLSN-probe-assisted FL imaging.

As numerous studies have proposed, the current QDs used as contrast agents suffer from weak dispersion in aqueous base solutions such as cell culture medium, and hence, effortless dispersion of a contrast agent in cell culture medium is ideal for bioimaging applications ${ }^{109}$. For PLSN-probeassisted bioimaging, two major excitation wavelengths, 350 and $490 \mathrm{~nm}$, were chosen since blue and green are the most common choices in FL microscopy. The observation revealed higher brightness of the dyes (FITC and DAPI) in the presence of the PLSN-probes. Moreover, PLSNprobe-assisted FL imaging displayed a longer stability of the dyes. As plotted in Figure 5.8 (C), DAPI and FITC photobleached after approximately 4 minutes of exposure. However, PLSNprobe-assisted FL imaging demonstrated a significantly higher stability of these two dyes. The FL images in Figure 5.8 (A-B), acquired after 10 minutes of exposure at excitation wavelengths of 350 and $490 \mathrm{~nm}$, still display details of the nucleus and cytoskeleton.

\subsection{Summary}

Here, we presented a novel approach using polyhedral PLSN-probes with a broad range of absorption and long Stokes shifts that exhibited multiplex emissions. These probes exhibit a diagnostic bioimaging property that allows one to differentiate between cancerous HeLa cells and mammalian fibroblast cells and to screen the health of cells without using organic fluorescent labels. This label-free method has shown a precise capability of cell uptake that strongly improves upon the lengthy labeling process of other methods. The multi-crystallinity of the polyhedral structure allows higher absorption for the PLSN-probe, and hence, a greater FL emission was observed. Broad absorption and narrow emissions with long Stokes shifts render these probes an efficient tool for bioimaging without the use of organic dyes. The superior uptake of the PLSNprobes within HeLa and fibroblast cells exhibited no need for conjugation. However, this attribute was found to be cell-selective, i.e., the HeLa cells demonstrated significantly extended internalization, whereas the fibroblast cells showed a weaker tendency to uptake the PLSN-probes. Unlike current bioimaging contrast agents, the PLSN-probes demonstrated excellent dispersion in aqueous cell culture medium and systematic division into smaller parts that internalize into 
different organs of the cell, including the nucleus, nucleolemma and cytoskeleton. PLSN-probes properly dispersed within a cell could be used for 3D visualization of intercellular organs of the cell. Moreover, we found that the FL intensity of the PLSN-probes per cell area increases upon shrinkage of the cytoskeleton of the cell. Hence, this characteristic allows one to conclusively differentiate healthy cells from dead cells. In PLSN-probe-assisted FL imaging, fibroblast and HeLa cells stained with DAPI and FITC depicted a lower photobleaching sensitivity, which shows the feasibility of using the PLSN-probes as a contrast agent. In addition, the proposed probes can be used to diagnose HeLa cells as well as to screen dead cells. Further investigations are required to study the interaction of different cell lines with these PLSN-probes. 


\section{CHAPTER 6}

\section{SURFACE ENHANCED RAMAN SPECTROSCOPY (SERS)-ENABLED POLYGONAL SILICON QUANTUM PROBE TOWARDS CANCER DIAGNOSIS}

Keshavarz, M.; Tan, B.; Venkatakrishnan, K. "In-situ cancer diagnosis with Surface Enhanced Raman Spectroscopy (SERS)-Enabled polygonal Si quantum-probes." Submitted to Jornal of Advanced Functional Materials.

\subsection{Abstract}

Accurate analysis of specific cellular substances is essential for early diagnosis and treatment of cancer. Here, we report on the unique approach of using a polygonal Silicon quantum probe (polygonal Si-QP) that utilizes a surface-enhanced Raman scattering (SERS) for diagnosis and differentiating of cancerous HeLa and mammalian fibroblast cells by intracellular sensing. The self-internalization, as well as label-free attributions of the polygonal Si-QPs, allow us to overcome the limitations of the conventional SERS-enabled platforms for live-cell Raman sensing. We demonstrated that in-situ live-cell analysis of cells has been made possible due to the angular morphology of an individual polygonal Si-QPs that drastically enhances a localized surface Plasmon. Our results showed that the SERS functionality of the synthesized polygonal Si-NPs not only allows the tracking of their internalization within a cell which reveals the fingerprint of substantial components and organelles of a cell but also to detect a cancerous HeLa cell. The monitoring of a cellular behavior over a course of 24 hours within $6 \mathrm{~h}$ interval, revealed new insights into substantial proteins in order for a cell to be viable. This unique attribution of the polygonal Si-QP relies on the multidimensionality of the Si-NPs driven by kinetically controlled self-assembly process during laser-induced ionization. Precise control of the ionization energy induced by ultrashort pulsed laser (USPL) resulted in a transition from spherical to polygonal SiNPs. 


\subsection{Introduction}

Cancer defined as uncontrolled cell growth resulting from several abnormalities in cellular pathways at the single-cell level among which cervical cancer is the second most common malignancy in women worldwide and yet the most treatable one if detected early. Therefore, we have chosen HeLa to proof of concept of using Raman spectroscopy by means of SERS-enabled polygonal Si-QPs for cancer diagnosis and differentiation ${ }^{1-4}$. Due to the complexity in its formation and the difficulty of early diagnosis, it remains as one of the leading causes of death in the developed world. The major obstacle to preventing the disease is not being able to diagnose cancer early enough. Most of the time, it is diagnosed after symptoms appear and it can be too late for an intervention $^{5,6}$. Therefore, early diagnosis is crucially important for the success of the treatment. In this study, HeLa cancer which is a disease with high mortality has chosen to diagnose and distinguished from fibroblast ${ }^{7}$.

Among the current diagnostic techniques, Raman spectroscopy (RS) is increasingly investigated for early cancer diagnosis. The potentials of this technique are slowly making its way into clinical cancer diagnosis ${ }^{8-9}$. There are increasing numbers of reports emerging that RS can help clinicians for early cancer diagnosis and eases the follow-up treatment. However, several challenges remain to be overcome. The high chemical specificity, minimal or lack of sample preparation and the ability to use advanced optical technologies in the visible or near-infrared spectral range (lasers, microscopes, and fiber-optics) have recently led to an increase in medical diagnostic applications of Raman spectroscopy ${ }^{10-13}$. The key hypothesis underpinning this field is that molecular changes in cells, tissues or biofluids, that are either the cause or the effect of diseases, can be detected and quantified by Raman spectroscopy ${ }^{12}$.

Raman spectroscopy is a vibrational spectroscopic technique and it provides chemical information from a molecule or molecular structure. Since the Raman spectrum of a molecule is a 'fingerprint' and the bands on a spectrum have a narrow bandwidth, a Raman spectrum from a molecular mixture can provide molecular level information about its chemical composition. In addition, it has low sensitivity for water ${ }^{10,14-16}$. Therefore, this technique is more suitable for the analysis of water containing samples with biological origins such as cells and tissues compared with conventional current methods such as IR spectroscopy, which is another well-established 
vibrational spectroscopic technique ${ }^{16-21}$. However, inherently low scattering efficiency of RS has hampered its utilization until the development of powerful lasers and sensitive detectors. Although the improvements in instrumentation have allowed the use of the technique for a wide range of applications and significantly reduced collection times, its low sensitivity continues to be a bottleneck, especially for some in vivo applications. The Raman spectroscopy in combination with therapeutic treatment can be a powerful technique for discriminating pre-cancerous and cancerous biochemical changes ${ }^{22-25}$.

As the applicability of the RS for cancer diagnosis has substantiated, several studies have tried to tackle the major drawback of this method which is less inelastic scattering by the emergence of surface-enhanced Raman scattering (SERS). SERS can also be used for the analysis of (Nano/Quantum scale) particle internalization. Furthermore, with the development of faster stages and line scan mode, where the laser beam can be extended into a line shape to raster the target area, it is now possible to further decrease the mapping time ${ }^{25-30}$. Although the mapping speed is not at the desired level, the Raman microscopy systems are now more applicable for the mapping of large areas (from $\mu \mathrm{m} 2$ to $\mathrm{mm} 2$ ) such as cells and tissues. SERS can be extremely sensitive however, the necessity to use colloidal NPs can be a potential problem since the NPs are not distributed evenly within the cell of interest. The arbitrary distribution of the NPs introduces additional uncertainties into SERS measurements ${ }^{32-35}$.

Since SERS can provide molecular level information about the composition of a sample, the gradual biochemical changes from a healthy tissue to a tumor is reflected in a Raman spectrum. From the sensitivity point of view, SERS can be exploited to enhance the Raman scattering. Enhancement in Raman scattering is the result of the coupling of electron system of a nanostructured surface and molecule located on the surface or in the close vicinity of the nanostructure surface, which results in increased polarize-ability of the molecule or molecular structure. It is now commonly accepted that two mechanisms of electromagnetic and chemical through surface plasmons and charge transfer, respectively, play role in SERS effect. Hence, using SERS has made detection of a single molecule possible ${ }^{36-40}$.

This increased sensitivity compared with the other modes of RS provides unique opportunities for detection of the especially low-concentration analyte. However, the fact that the molecule or molecular structure should be brought to the surface or to the very close vicinity of a nanostructure such as gold and silver SERS-active platform can limit the technique for certain applications such 
as intracellular investigation. Since the discovery of SERS phenomenon, a significant amount of effort has been devoted to in-situ exploring its potential for a variety of applications including intracellular detection of proteins, DNA, microorganisms, eukaryotic cells, and tissues ${ }^{41-45}$.

SERS retains the chemical and structural information provided by Raman spectroscopy but to overcome the intrinsic weakness of the Raman scattering process the polygonal Si-QPs were synthesized and exploited. Due to the facial indentation of the polygonal Si-QPs, localized surface plasmon resonances (LSPRs) further enhanced the surface-enhanced resonance Raman scattering (SERS). Additionally, the advantages of evenly distribution, nontoxicity, higher absorption in the optical window region and affinity to function groups have made the polygonal Si-QP an excellent probe for in situ diagnosis $45-50$.

In order to overcome this obstacle, we herein reported SERS-enabled quantum scale-probe that is being uptake by a cell without a means of bioconjugation. Unlike the conventional nanostructured platform that is SERS active, the polygonal Si-NPs internalize a cell of interest and has shown an exceptional localized Raman detection. We have demonstrated the feasibility of RS for cell differentiation which has made cervical cancer cells diagnosis possible. Extensive multivariate algorithms, however, are necessary to distinguish the subtle differences between the healthy tissue and cancerous tissue at very early stages. The expectation is that the technique can detect the changes at the earliest possible point, and then a step toward treatment can be taken. The advancement in fiber optic could also make RS possible for an in-vivo diagnosis. However, abandon from restrictive SERS-active platforms are essentials ${ }^{50-52}$.

In ex vivo applications, Raman Mapping provides an image of the cell at chosen wavelength that can provide intrinsic biochemical information that can be used for diagnosis. In this study, the identification of the HeLa in comparison with fibroblast was investigated. The RS of each cell lines were inspected after 6 hours over a course of 24 hours then the results acquired from Raman mapping of a single cell were used as a reference for identification of individual components. Multivariate analysis of principle component analysis (PCA) and Linear Discriminant Analysis (LDA) was used to extract information from the entire dataset collected from SERS spectra. It was concluded that HeLa and fibroblast could be differentiated, after an extensive statistical treatment of the data. Moreover, based on behavioral analysis of cells, normal (healthy) from ones undergoing death could be predicted which ultimately can lead to an algorithm to study the effectiveness of therapeutic cancer treatment. 


\subsection{Materials and methods}

\subsubsection{Synthesis of the SERS-enabled polygonal Si-QPs}

The polygonal Si-QPs were generation by employment of a Clark-MXR IMPULSE pulsed Ybdoped fibre amplified femtosecond laser to ionize a $0.02-\Omega \mathrm{cm}$ N-type silicon (100) crystallographic wafer. The utilized laser is capable of producing central wavelengths of $1040 \mathrm{~nm}$ with a pulse width ranging from 214 fs to $1428 \mathrm{fs}$, as well as a tunable laser pulse repetition rate that can vary between $4 \mathrm{MHz}$ and $26 \mathrm{MHz}$. Prior to the synthesis the wafer was sliced into 2-cm2 square-shaped samples and ultrasonically cleaned in a $50^{\circ} \mathrm{C}$ acetone bath for 15 minutes, followed by rinsing and drying. The sequential multiphoton ionization was initiated by laser irradiation of single crystalline Silicon chip. For the purpose of this study; average laser power, repitation rate (rep.rate) and laser pulse width has set to $16 \mathrm{~W}, 26 \mathrm{MHz}$ and $214 \mathrm{fs}$. An inert atmosphere condition applied to prevent oxidation of the synthesized Si-QPs. Utilizing the pulsed laser (USPL) is advantageous since the absorption of multiphoton excitation energy overcomes the ionization threshold of the Silicon chips. Moreover, given the localized laser beam spot $(10 \mu \mathrm{m})$ within the focal point, results in precise control over the synthesized Si-QPs. Therefore, irradiated at the beam focal point, with a computerized galvano scanner precisely implementing the synthesis processes.

\subsubsection{Characterization techniques}

The synthesized Si-QPs investigated in terms of the morphology with a JEOL JSM-4800 field emission scanning electron microscope (FE-SEM). The images were taken at $10 \mathrm{kV}$ accelerating voltage. The size distribution and morphology of the Si-QP was characterized by using a JEOL JEM 2100 high-resolution transmission electron microscope (HR-TEM). The HR-TEM experiment carried by suspending the synthesized Si-QP in ethanol and placing a droplet on a copper grid for direct observation after drying. The particle size measurement was carried on by exploiting an image analysis software. Moreover, the crystallinity of the Si-QP was determined by utilizing an HR-TEM diffraction pattern (FFT). The elemental analysis was performed by Energy dispersive X-ray spectra (EDX) analyses using Oxford Instruments. Ultraviolet-visible-near- 
infrared (UV-Vis-NIR) absorption was obtained with a Hitachi UV-3100 UV-Vis-NIR spectrophotometer.

\subsubsection{Raman spectroscopy analysis}

The Raman spectroscopic analysis was performed using a micro-Raman spectrometer (Renishaw inVia Confocal Raman Spectrometer) equipped with a Leica DMI6000 automated epifluorescence microscope and with 3 solid state excitation sources $(532 \mathrm{~nm}, 638 \mathrm{~nm}, 785 \mathrm{~nm})$ and a deep-depletion silicon CCD detector which has sensitivity from approximately 400-1000nm. The confocal Raman microscope allows spatially resolved spectroscopy to be carried out in a confocal manner at high speed. The laser source $(785 \mathrm{~nm})$ was focused on the sample through a $20 \times$ objective lenses. Data analysis was performed using the Renishaw Wire software.

\subsubsection{Cell culture and seeding}

Prior to their addition to cell culture medium, the synthesized Si-QPs were sterilized under UV light for 20 minutes. Human cervical cancer cells (HeLa, ATCC, American Type Culture Collection, ATCC No. CCL-2) and Fibroblasts (NIH3T3) were employed in cell experiments to ascertain the differences of mammalian and cancer cell lines in response to their biochemical fingerprints. Fibroblast cells were grown in DMEM containing 10\% heat-activated fetal bovine serum with $1 \%$ penicillin-streptomycin antibiotics (Pen-strep). HeLa cells were grown in DMEMF12 supplemented with $10 \%$ fetal bovine serum and 1\% Pen-strep. Subsequently, the cells were separately cultured on substrates placed in Petri dishes with a seeding density of 750,000 cells/cm2. The Petri dishes were placed in an incubator for 24 and 48 hours at $37{ }^{\circ} \mathrm{C}$.

\subsubsection{Fluorescence microscopy}

For FL microscopy, the samples were first fixed in methanol-free paraformaldehyde followed by incubation in skim milk to prevent non-specific binding. To stain the actin and cytoskeleton, the samples were incubated with Alexa Fluor 488 (Life Technologies) followed by DAPI (Life Technologies) to stain the nucleus. The samples were studied using a FL microscope (Nikon, 
Canada). Seeded cells were also fluorescence-imaged on a Zeiss LSM 710 META upright confocal laser-scanning microscope.

Statistics; All experiments were performed in triplicate, and the data points were averaged unless otherwise indicated. The error bars show standard deviations.

\subsubsection{Data analysis}

Principal component analysis (PCA) which is capable of analyzing complex sets of data with multiple variables was employed. PCA performed on acquired Raman spectra where n-by$\mathrm{p}$ matrix ( $\mathrm{n}$ spectra with $\mathrm{p}$ data points) represents a Raman intensity spectrum and the wavenumber. The PCA aimed to find a unique p-dimensional orthogonal coordinate system where the projection of data on each coordinate axis has a sequentially maximal variance. Hence, each projection corresponds to a linear combination of the original data, while the first projection having the maximum variance and representing the first principal component.

\subsection{Results and discussion}

\subsubsection{Fabrication and characterization}

Development of Si-QPs as an in-situ method for intracellular monitoring and cell diagnosis greatly benefited from unique Localized Surface Plasmon (LSP), which ascribed to the exterior polygonal structures of the quantumized Silicon particles. The polygonal Si-QPs were synthesized by femtosecond laser processing of silicon (Si) chips, as depicted in the schematic in Figure 6.1. The unique attribution of the synthesized polygonal Si-QP relies on multidimensionality of the Si-QPs driven by kinetically controlled self-assembly process during laser-induced ionization. Precise control on the ionization energy induced by ultrashort-pulsed laser (USPL) resulted in transition from spherical to polygonal Si-QPs. Angular morphology of the polygonal Si-QPs as depicted in Figure 6.1 (a-c) allows overcoming the limitations of the conventional SERS-enabled platform by which intercellular spectroscopy of individual cell has made possible. 

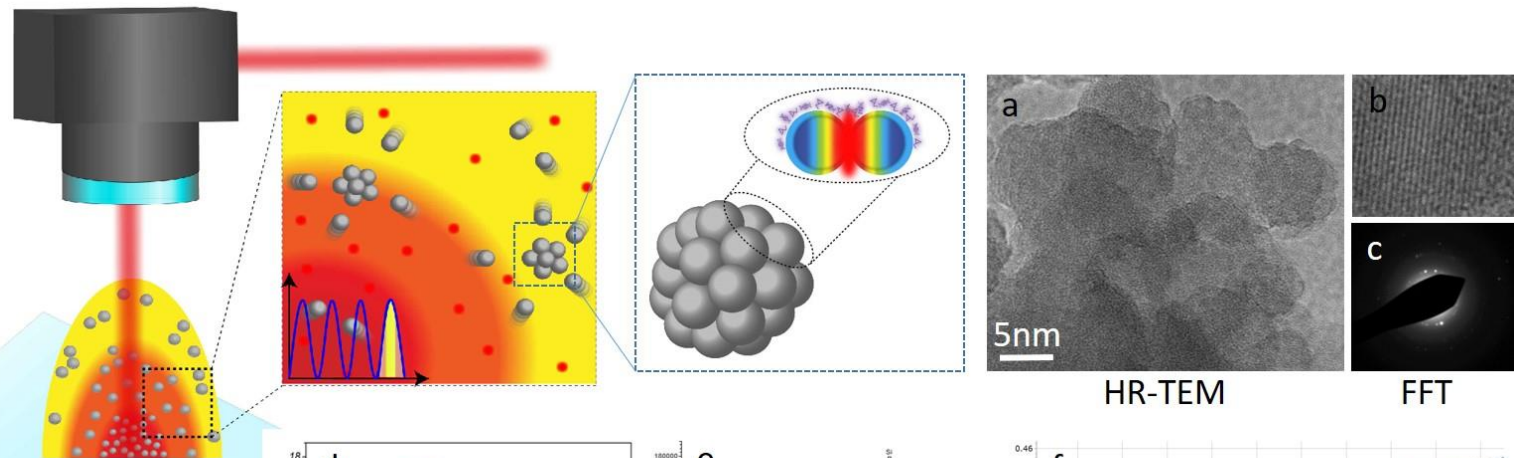

HR-TEM

FFT

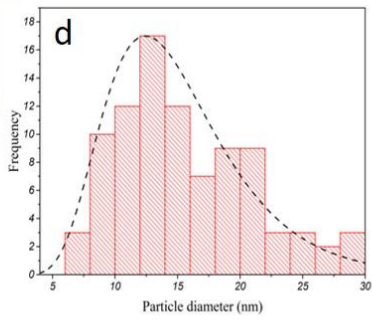

Particle size distribution

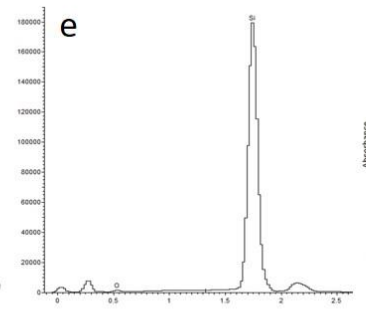

EDX spectrum

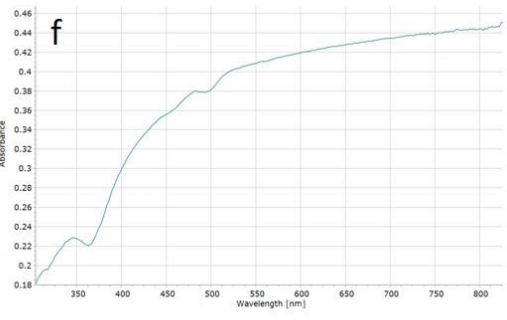

UV-Vis Absorption

Figure 6.1: Schematic illustration showing the synthesis of the polygonal Si-QPs. TEM micrograph (a) exhibits the polygonal morphology of individual particles. HR-TEM crystal d-spacing and diffraction patterns (b-c) demonstrate the crystallinity of the synthesized polygonal Si-QPs. Size distribution plotted in histograms shows 5-20nm range. (Statistical analysis performed over 1000 particles with SD of 0.63). EDX spectrum (d) represent the formation of pure Silicon particles. The UV-Vis spectrum shows higher absorption at longer wavelength.

The synthesized polygonal Si-QPs possess facial indentation where the so-called SERS hot spots can be formed. Here the synthesis of polygonal Si-QPs with average diameters of 5-20 nm made of facial indentation is reported (Figure 6.1 (d)). The ultra-short laser at femtosecond regime has been used to synthesis the polygonal Si-QPs. The sequential multiphoton ionization was initiated by laser irradiation of (100) single crystalline Silicon substrate. To overcome the ionization threshold of the Silicon, average laser power, repitation rate (rep.rate) and laser pulse width has set to $16 \mathrm{~W}, 26 \mathrm{MHz}$ and $214 \mathrm{fs}$. The Simultaneous interaction of ultra-high laser rep.rate at narrow pulse width has only given a time for embryonic initiation of Si-QPs this process however, was disrupted by pulsed trains of laser irradiation, which caused nonuniform self-assembled polygonal Si-QPs. The oxidation of the synthesized Si-QPs was prevented at inert atmosphere condition. The EXD elemental analysis (Figure 6.1 (e)) prove the prevention of chemical changes during the synthesis.

In order to achieve intensified scattering intensities, it is essential that the Raman excitation wavelength be chosen in the electronic transition region (UV-visible area). Hence, detection limits 
and measurement times can be significantly decreased. However, the coincidence of the excitation and fluorescence backgrounds at UV-visible can be significant. The advantage of Surface Enhanced Raman Scattering (SERS) approach is that, fluorescence background is suppressed whilst the Raman intensity of an analyst is enhanced. As shown in Figure 6.1 (f), the Si-QP has a strong absorption band in the UV-VIS region. The strong absorption band appears when the incident photon frequency is in resonance with the collective oscillation of the conduction electrons. This phenomenon resulting in Raman signal enhancement of the adsorbed molecules on the surface of the polygonal Si-QPs. The proximity between the facial indentations makes them excellent supports for (SERS) which demonstrated an excellent photon absorption at visible wavelengths. The high mobility of electrons in semiconductor nanoparticles leads to high absorption and plasmon resonances in UV-Vis range, which makes them uniquely suitable for a Raman sensing. This attribution is ideal for clinical use due to the high absorption at wavelengths in the "optical window", such as 785nm, at which they generate minimal background fluorescence and yet quantum-scale polygonal Si-QPs penetrate deeply into the cells. These properties combined with non-toxicity of silicon-based materials to mammalian cells can spurred their application for diagnostics by SERS. Development of SERS capabilities for each individual particle makes multiple applications possible including in situ monitoring of biological processes and physiological changes inside the cells in real time.

The SERS-enabled polygonal Si-QPs has shown high ability to enhance the Raman signal in the study of biological compounds to very low concentrations even single molecules. Thus, the polygonal Si-QPs has been applied to detect the biochemical components inside the HeLa and fibroblast living cells. The synthesized polygonal Si-QPs takes the advantage of being labeled-free and yet internalize into the cells. However, the affinity of this probes to self-conjugate in present of cell culture media evidenced in the Figure 6.2. As depicted in the Figure 6.2, polygonal Si-QPs (red spectrum) consist of a band around $784 \mathrm{~cm}^{-1}$ which refers to a symmetric stretching vibration of $\mathrm{Si}-\mathrm{O}-\mathrm{Si}$ as well as a strong band at around $1075 \mathrm{~cm}^{-1}$ wavelength, corresponds to the asymmetric stretching vibration of $\mathrm{Si}-\mathrm{O}-\mathrm{Si}$ that indicates a robust cross-linked framework. These bands of the inorganic frameworks were observed on the polygonal Si-QPs after modification. However, the FT-IR spectrum of the self-modified polygonal Si-QPs demonstrated a band at $3389 \mathrm{~cm}^{-1}$ corresponded to the $\mathrm{N}-\mathrm{H}$ and $\mathrm{C}-\mathrm{H}$ stretching vibration. Since this band was not 
observed in the spectrum of the as synthesized polygonal Si-QPs, this result was sufficient to give clear evidence that organic amino groups were grafted onto the surface of the polygonal Si-QPs.

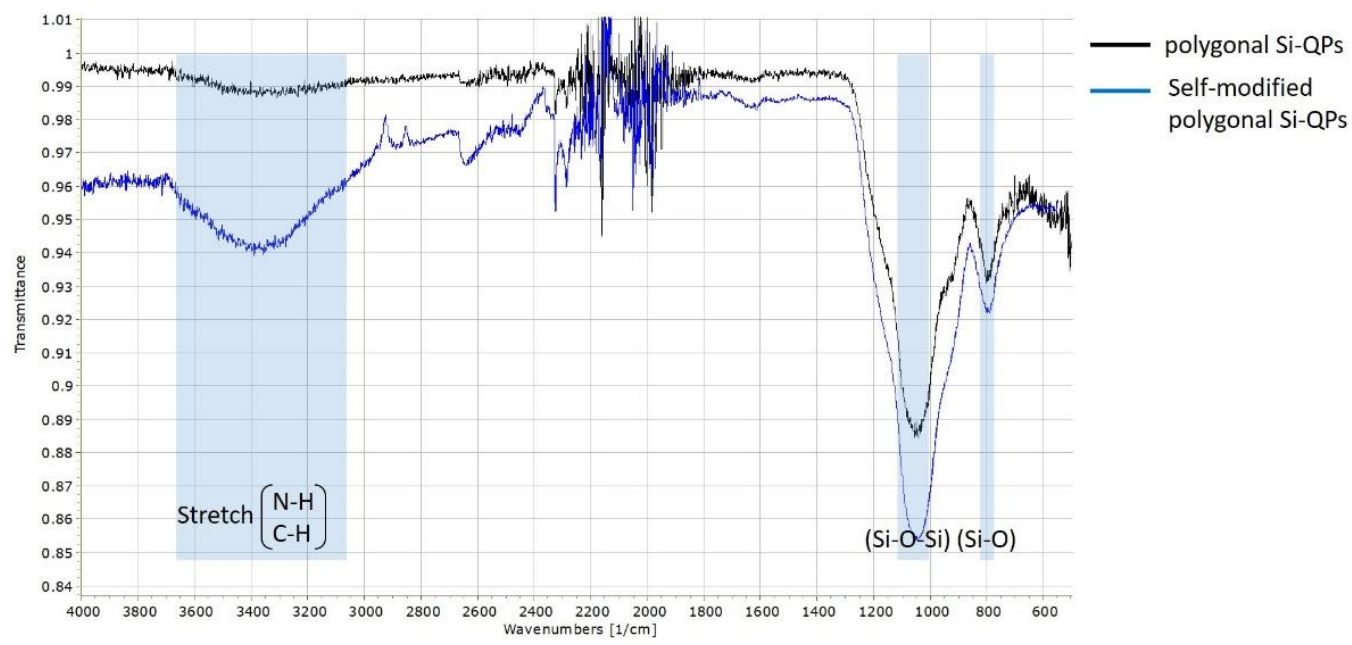

Figure 6.2: Fourier transform infrared (FT-IR) spectroscopy confirms the grafting of amides on the surface of the polygonal SiQPs. The FT-IR spectra (Figure 6.2) displays as synthesized spectrum (red) of the polygonal Si-QPs, after modification (green) and (blue) the cell culture media.

Considering that the composition of the healthy and cancerous cells differs at the molecular level and this difference can be reflected in the Raman spectrum. When a reporter molecule is attached to the polygonal Si Q-probe, the Raman scattering from a molecule in its vicinity is significantly enhanced. Therefore, preferential interaction of certain functional groups such as -SH, -HN2 and $-\mathrm{COOH}$ with the Si-QPs resulted in the spectra dominated with the bands originating from the molecular structures possessing these moieties.

\subsubsection{Raman spectroscopy for live single cell imaging}

The wavelength of a Raman laser used for excitation of biomolecules plays an important role. Shorter Raman wavelength such as 532nm generates higher numbers of scattering photons, therefore, enhances the Raman intensity of the weakly scattering biological samples. However, shorter wavelengths stimulate more autofluorescence from the sample, potentially exceeding the Raman signal. Therefore, an adequate laser wavelength should be considered since light absorption of a biological sample (cells) can cause a photo-damage. We found that an excitation wavelength 
of $785 \mathrm{~nm}$ is a good compromise, as signal strength is reasonably high, whereas fluorescence and cell damage are low. For similar reasons, this wavelength is often used in Raman studies analyzing tissue especially skin. Additionally, light from the near infrared region penetrates sufficiently deep into tissue.

The major building block molecules of a cell comprise proteins, nucleic acids, lipids among others. Molecular vibrations deriving from the functional groups of these building blocks define the Raman spectrum of cells. A Raman spectrum can be separated into three parts: the fingerprint region (less than $1800 \mathrm{~cm}^{-1}$ ), the silent region $\left(1800-2800 \mathrm{~cm}^{-1}\right.$ ) and the high frequency region (over $2800 \mathrm{~cm}^{-1}$ ). The molecular fingerprints of the functional groups can be found at 600 to $1800 \mathrm{~cm}^{-1}$ and above $2800 \mathrm{~cm}^{-1}$ (mostly for lipids) which is dominated by $\mathrm{CH}$-stretch vibrations. Due to the high affinity of the polygonal Si-QPs to C-H bonds as evidenced in FITR (Figure 6.2) a variety of components in this region were identified. In addition, the characteristic pack Silicon at around $521 \mathrm{~cm}^{-1}$ does not interfere with the finger print region. Hence, the polygonal Si-QPs shows the excellence capability for cellular biomolecular detection. Figure 6.3 shows an ex vivo example of Raman spectra from a normal HeLa cell. Since, the vibrational microspectroscopy used in this experiment is contingent on molecular vibrations emerging from functional groups within the cell, the compositional mapping carried out without a need for labelling. Depending on the nature of the cell and the physicochemical characteristics of the Nano/quantum particles being used, the dominant uptake is endocytic pathways. Almost every cell type is able to uptake particles via an endocytotic pathway. However, the extent varies from well distribution of particles within the cells. The synthesized polygonal Si-QPs have shown superior permeability to self-internalize into the cell and plays its role as in situ enhancer of Raman scattering.

Raman spectra of the main molecules present in cells proteins, nucleic acids, and lipids are shown in Figure 6.4 and 6.6. Furthermore, an overview about important band positions and cell related functional groups is given in (Supplementary Table 1). The Raman spectra of biological molecules as listed in Table 1 gives a summary of some common biochemical functional groups with their molecular vibrational modes and frequency ranges, which are observed in compounds such as proteins, lipids, and nucleic acids. The supremacy of polygonal Si-QPs to identification of these functional groups can be used as a qualitative or quantitative assessment of a change or anomaly in a sample under investigation in this case HeLa fibroblast. Listed wavenumbers however, may vary as the given peak position is directly related to the resolution of the used spectrometer. 

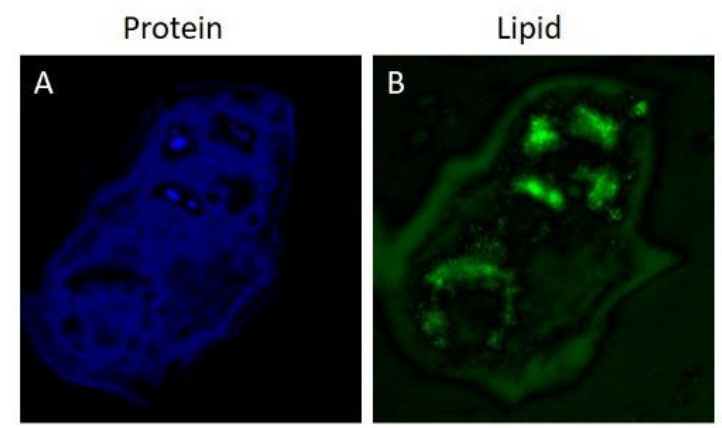

Nucleus Acid- Cyto
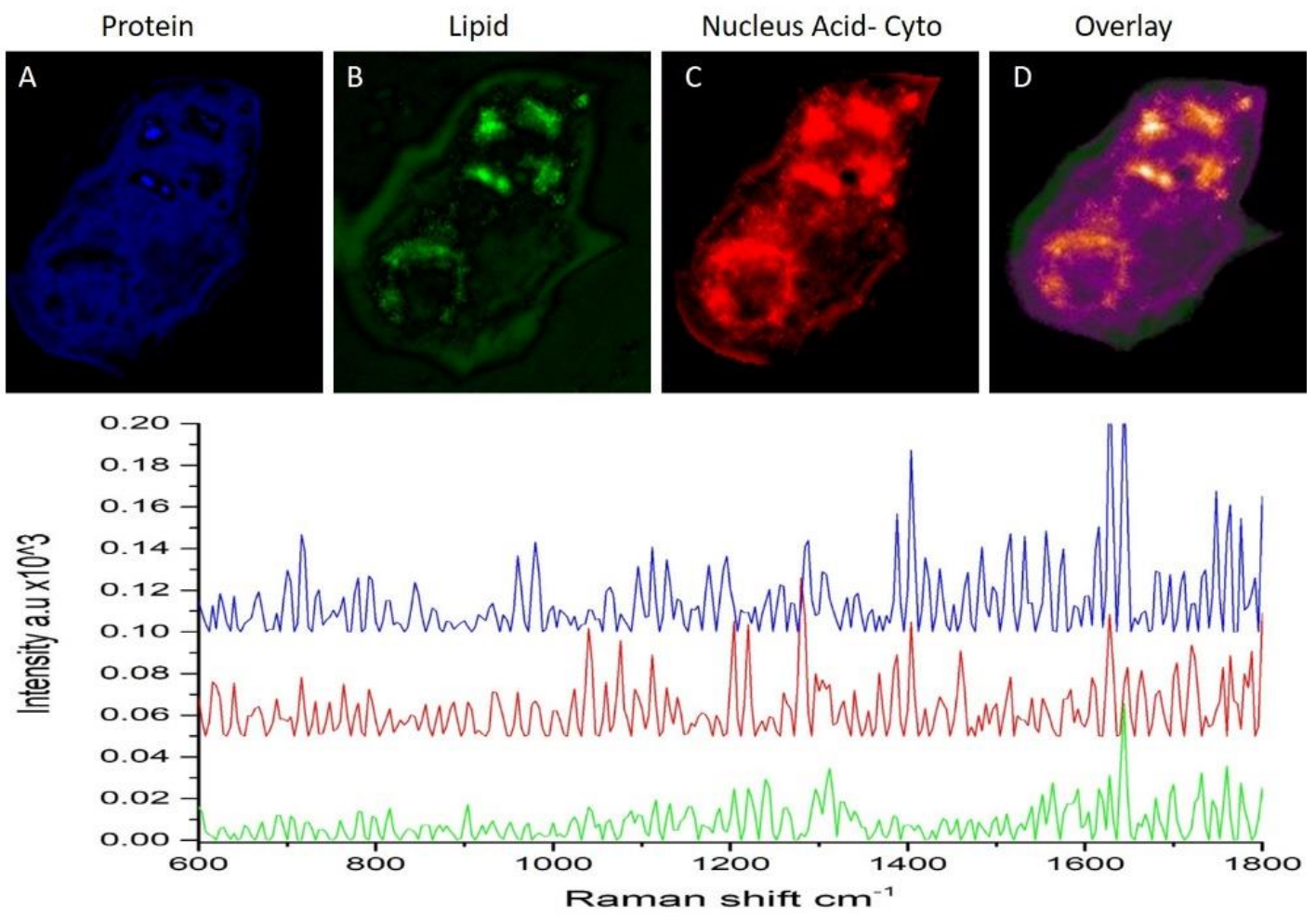

Figure 6.3: Raman mapping of single HeLa cell. (A-C) exhibit the protein, Lipid, and Nucleic acid distribution in the cytoskeleton of a HeLa cell. (D) Shows the overlay of detected substances. SERS activated spectrum of depicted healthy HeLa cell disclose the dominance of Protein, Lipid, and Nucleic acid in different organelle.

\subsubsection{Cell physiology}

The attempt of utilizing Raman spectroscopy for imaging living cells is relatively new. One of the advantages of Raman microscopy is its capability for label-free imaging by detecting vibrations of molecules. However, it is quite a challenge for Raman microscopy to observe a living cell. The surface-enhanced Raman scattering (SERS) enabled Si-QPs has an advantage of localized surface plasmon (LSP) introduced by its polygonal morphology. For the parallel detection of Raman spectra, a line-shaped focus illuminated the sample instead of a single laser focus used in a conventional confocal Raman spectrophotometer by which several hundreds of points in the sample were measured simultaneously. Consequently, the image acquisition time decreases proportional to the number of detection points. The $785 \mathrm{~nm}$ near-infrared (NIR) laser was utilized 
and Raman scattering from the sample was recorded by a spectrophotometer. Raman spectroscopy in which NIR excitation minimizes fluorescence and absorption by tissue, is the ideal choice for potential in vivo applications.

The Raman spectrum was measured from the cytosol of a living HeLa cell (Figure 6.3). The distribution of Raman spectra in a sample is measured and the intensity of the peaks of interest mapped to construct a Raman image. The Raman images of a living HeLa cell are shown in color channels where blue, green, and red represent the distribution of proteins, lipids and nucleic acid respectively. The overlay image was constructed by assigning the different Raman images to different color channels. In this experiment, the Raman spectra exhibited narrow peaks, especially in the fingerprint region of $600-1800 \mathrm{~cm}^{-1}$. The self-internalized polygonal Si-QP has provided a broad range biochemical information about a sample, including composition and concentrations of constituents, identified peaks associated with specific vibrations in molecular bonds are tabulated in appendix 1. The Raman peaks assigned to vibrational modes verified by literatures. The Raman peak at $720 \mathrm{~cm}^{-1}$ assigned to a vibrational mode of the pyrrole ring in cytochrome $\mathrm{c}$, which is crucial in ATP generation. Cytochrome c absorbs light at around $700 \mathrm{~nm}$ and therefore shows a strong Raman scattering because of resonant absorption of the 785nm excitation light. Since cytochrome $\mathrm{c}$ is localized in mitochondria, the distribution observed at $720 \mathrm{~cm}^{-1}$ is evidently that of mitochondria presented in blue channel (Figure 6.3 (a)). The $1659 \mathrm{~cm}^{-1}$ band assigned to amide I vibrational mode of peptide bonds. The amide I appeared as a relatively wide band, and the larger Raman shift of the band typically shows vibration of peptide bonds in protein beta sheet. Therefore, the image at $1659 \mathrm{~cm}^{-1}$ provides contrast of proteins that distribute in the entire cell body (Figure 6.3 (A)). The $2855 \mathrm{~cm}^{-1}$ band assigned to symmetric stretching vibration of $\mathrm{CH} 2$. Since $\mathrm{CH} 2$ is richly contained in phospholipids, (Figure 6.3 (B)) the image contrast mainly shows lipid droplets and membranes of cell organelles such as mitochondria and Golgi apparatus. The image acquired with an exposure time of 120 s/line. Figure 6.3 shows Raman images obtained from a HeLa cell during the final stages of cell division. From the distribution of proteins given mainly at 1659 and $1404 \mathrm{~cm}^{-1}$, the division of cell body can be observed. The transposition of mitochondria and lipid droplets associated with the cell division is also visible. As shown in Figures 6.3, Raman mapping of lipid and nucleic acid on perinuclear region demonstrate a significant $\mathrm{CH}$-stretch bands around $2850-2900 \mathrm{~cm}^{-1}$, arising from the alkane chains of lipids. Additionally, presence of a peak at $715 \mathrm{~cm}^{-1}$ which potentially originated from the choline group 
of phospholipids abundant in membranes has revealed the confinement of nucleus and a cell boundary.
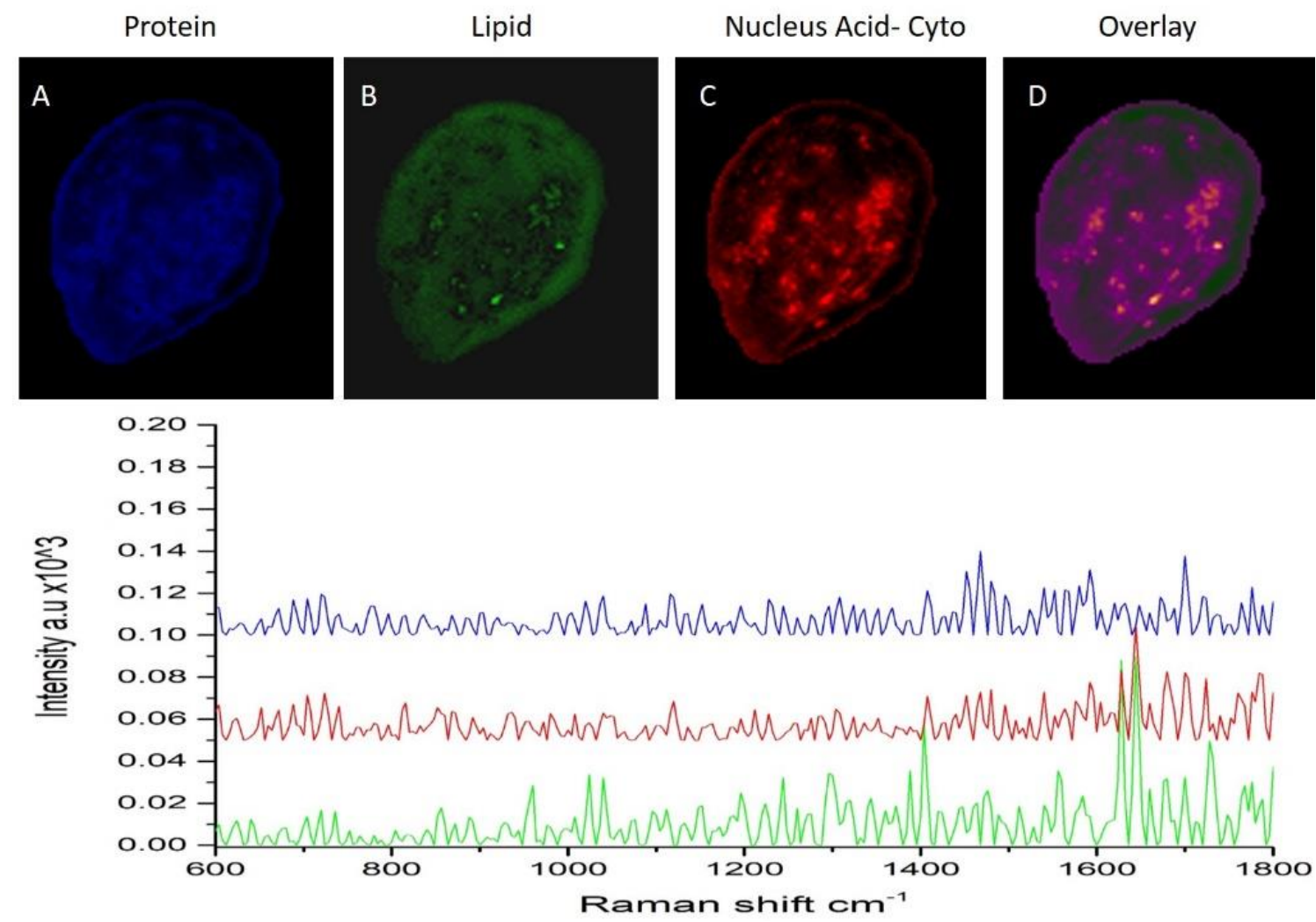

Figure 6.4: Raman mapping of apoptotic HeLa cell displays the disintegration of organelle. (A-C) exhibit the protein, Lipid, and Nucleic acid distribution in the cytoskeleton of a HeLa cell. (D) Shows the overlay of detected substances. SERS activated spectrum of depicted HeLa cell reveal the dominance of Protein, Lipid, and Nucleic acid in different organelle.

\subsubsection{Cell differentiation}

The ability of Raman to differentiate a live/dead state (cell viability of mammalian eukaryotic) was examined for HeLa cell. Significant differences in spectra relate to changes in the amount of DNA measured, as well as lipids and proteins. Figure 6.4 exhibits Raman images of a HeLa cell undergoing death. The Raman signal from nucleic acid manifests the fragmentation of DNAs (Figure $6.4(\mathrm{C})$ ). It believed that cytochrome $\mathrm{c}$ released from mitochondria to cytosol on the process of a cell death activates caspase cascades to decompose proteins in the cell. Hence, timelapse Raman imaging of a dead HeLa cell revealed that concentration of the cytochrome c 
drastically decreases in a dead cell. In addition, we found that the Raman band assigned to the amide I $\left(1659 \mathrm{~cm}^{-1}\right)$ disappears during the early dying process of a HeLa cell, much in advance of the morphological change of the cell.

A Cytoskeleton to nucleus ratio

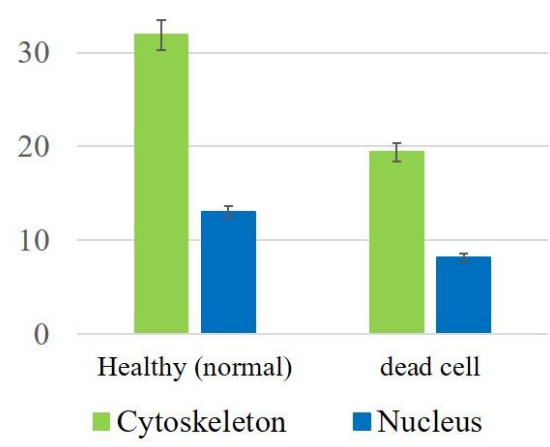

$\mathrm{D}$

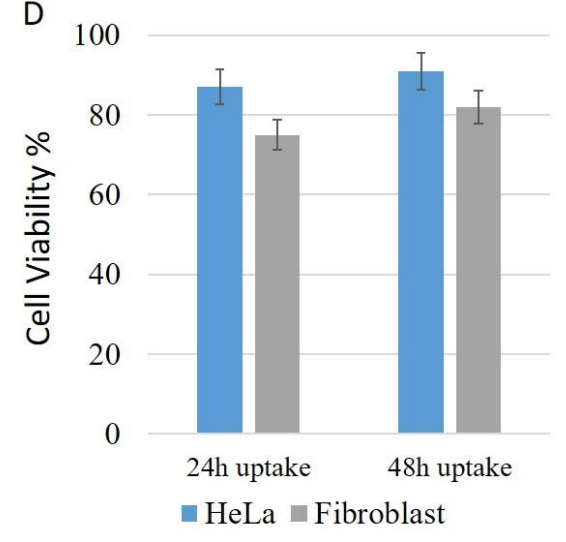

Fibroblast

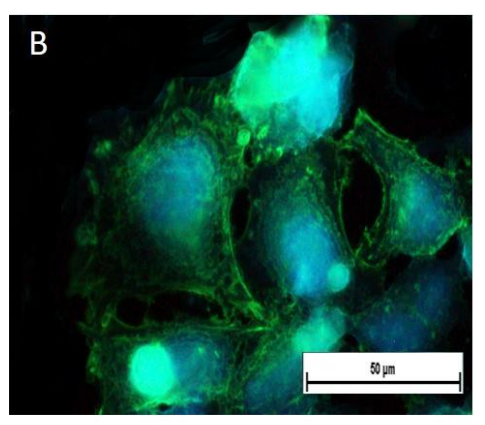

E 100

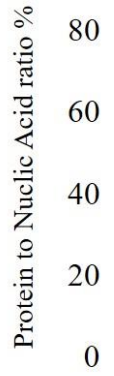

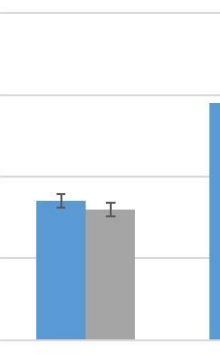

$6 \mathrm{~h}$

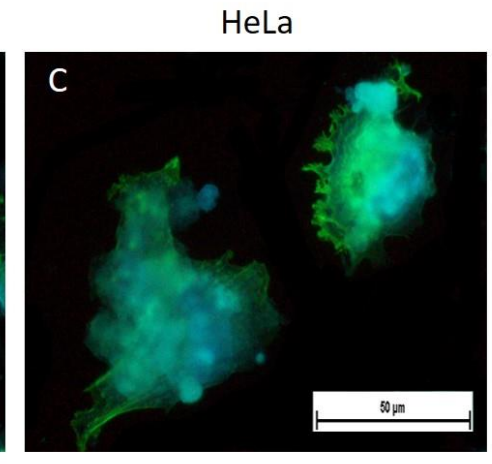

$12 \mathrm{~h}$

$\square$ HeLa $\square$ Fibroblast

Figure 6.5: Statistical analysis shows the ratio of cytoskeleton to nucleus ratio (A). Fluorescence images of fibroblast (B) and HeLa (C) stained with green (FITC) and blue (DAPI). (D) Shows cell viability assessment of HeLa and fibroblast after 24 and 48 hours. The ratio of protein to nucleic acid assessed by Raman spectroscopy (E) shows the nucleus of HeLa increase significantly compared to fibroblast.

The cell transition from normal state (control) to death was monitored, as shown in Figure 6.4, the ratio between nucleic acid, protein and lipid peaks has decreased. In particular, absence or drastic decent of proteins mainly Amino acids containing phenylalanine (980-1010 $\left.\mathrm{cm}^{-1}\right)$ and tyrosine $\left(1176,1627 \mathrm{~cm}^{-1}\right)$ along with nucleic acids $(\mathrm{G})\left(1320,1420 \mathrm{~cm}^{-1}\right)$ has found to be the indications of cell death. Raman spectrum of nucleic acid (red spectrum) showed the signals of the DNAs intensity were $\sim 4-5$ fold higher in healthy HeLa cell and that of dead cell. This is hypothesized to be caused by the DNA fragmentation of dead cells. Changes in Raman spectra of nucleus and 
cytoplasm were monitored within 6 hours interval for 24h. Changes of organelles and cell morphology upon cell dead was displayed in comparison to healthy cell states. Raman imaging (Figure 6.4) exhibits the disintegration of nucleus. Furthermore, the intensities of RNA $\left(813 \mathrm{~cm}^{-1}\right)$ and DNA $\left(786 \mathrm{~cm}^{-1}\right)$ have decanted as well. The decrease in RNA peak suggest the usage of RNA for protein synthesis during early cell division stages, whereas a lower intensity of the DNA peak indicates a reduced proliferation rate of the cells upon development of a mature phenotype. A concurrent decline of both RNA and DNA implies that the cell is dead. This is in accordance with fluorescence images taken form HeLa cells, as shown in Figure 6.5 (A), whose ratio of cytoskeleton of nucleus has drastically decreased on dead cells. By integrating the DNA bands intensity across individual cell nuclei, the Raman mapping corresponds to fluorescence image of stained nucleus. Mitochondria rich areas in pseudo color Raman maps of HeLa cells (Fig. 6 D-F) are congruent with fluorescence images of the same sample (Fig. 6 A-C), where the mitochondria were stained with FITC. The ratio of cytoskeleton of nucleus measured after cells were stained with DAPI and FITC dyes. The single cell Raman spectroscopy was taken as a representative signature for a number of cells. After an increase in lipid peaks (1266, 1303, 1445, 1656, and $1740 \mathrm{~cm}-1)$, an intensity increases in DNA Raman peaks (785, 1092, 1340, and $1578 \mathrm{~cm}-1)$ was detected followed by changes in DNA (signal decrease of peaks at 785, 1092, 1340, and $1578 \mathrm{~cm}^{-1}$ ) and protein Raman vibrations (increase of phenylalanine band at $1004 \mathrm{~cm}^{-1}$ ). Therefore, proliferative behavior or HeLa and Fibroblast based on the intensity changes if the Raman spectra were in accord with statistical analysis as plotted in Figure 6.6. Moreover, cell viability assessment of HeLa and fibroblast after 24 and 48 hours uptake evidenced nontoxicity of the polygonal Si-QPs. Furthermore, the cytoskeleton to nucleus ratio measured by staining with FITC and DPI are analogous with protein to nucleic acid ratio measured by Raman mapping. To select the peaks for this comparison, 20 percent of the highest intensity were chosen as a minimum threshold. The results of Raman spectroscopy agreed with standard fluorescence measurements. 

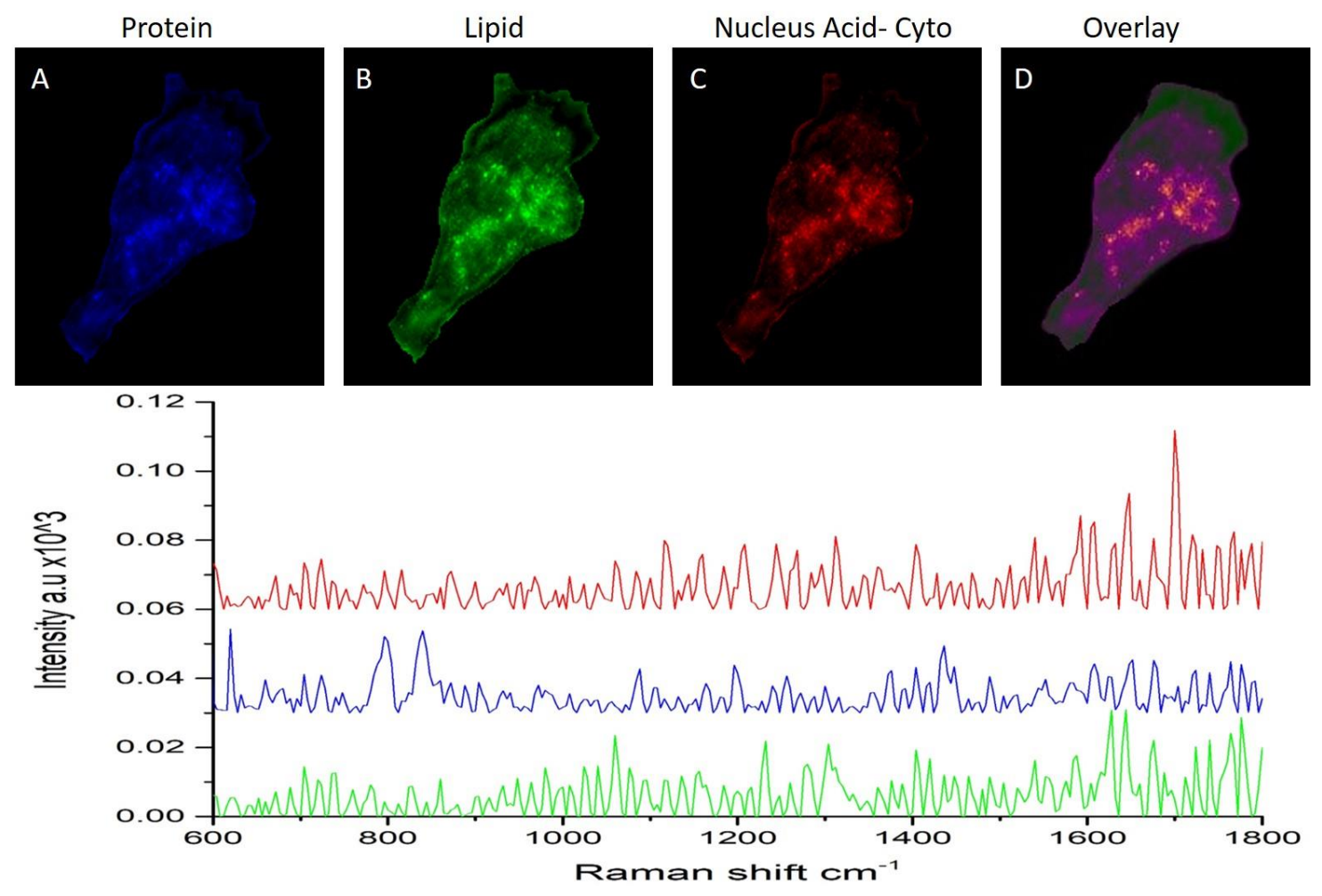

Figure 6.6: Raman mapping of unstained, unlabelled mammalian fibroblast (NIH3T3) cell displays the disintegration of organelle. (A-C) exhibit the protein, Lipid, and Nucleic acid distribution in the cytoskeleton of a HeLa cell. Overplayed image (D) constructed by merging images (A-C) with color channels. SERS activated spectrum of depicted HeLa cell reveal the dominance of Protein, Lipid, and Nucleic acid in different organelle. The sample was irradiated with a light intensity of -- $\mathrm{mW} / \mu \mathrm{m} 2$ at the focal plane in 78 lines of exposure. The exposure time of each line was $180 \mathrm{~s}$, and the images consist of $78 \times 281$ pixels.

\subsubsection{Diagnosis of HeLa cancer}

Different protein, nucleic acid, and lipid distribution in the two cells types were shown in Raman images made at a high frequency of protein, nucleic acid, and lipid bands. Subsequently 3 channels given to each category illustrated the distribution of these components in mapping images; normal (healthy) HeLa cell (Figure 6.3), a dead HeLa cell (Figure 6.4) and single fibroblast cell (Figure 6.6).

It was further shown, that lipid distribution was identical for images obtained from the high frequency bands (2800-3030 $\left.\mathrm{cm}^{-1}\right)$ and from the low frequency bands (1433-1481 $\left.\mathrm{cm}^{-1}\right)$. However, analysis of a protein/lipid system due to the overlap of the band derived from the phospholipid vesicle and the peak for protein image reconstructions at 1440 and $1451 \mathrm{~cm}^{-1}$, respectively. 
Identical biomolecular distribution was also shown in Raman images where a same pixel represents either or both protein, nucleic acid and lipids of HeLa and fibroblast cells. Raman mapping of single fibroblast and their subcellular structures are shown in Figure 6.6.

Raman bands in individual spectra were first assigned to nucleic acids (DNA and RNA), lipids (cholesterol and phospholipids) as well as to proteins and channeled in red, green, and blue colors respectively. Then, each spectrum was constructed by multivariate approaches, which were proven to be superior to univariate methods due to resolving band overlaps of cell constituents. Furthermore, as exhibited in Figure 6.6, a fibroblast cell was imaged in anaphase, before mitosis. The location of nucleus and vesicles within the cytoplasm and the appearance of microtubules were depicted in Figure 6.6.

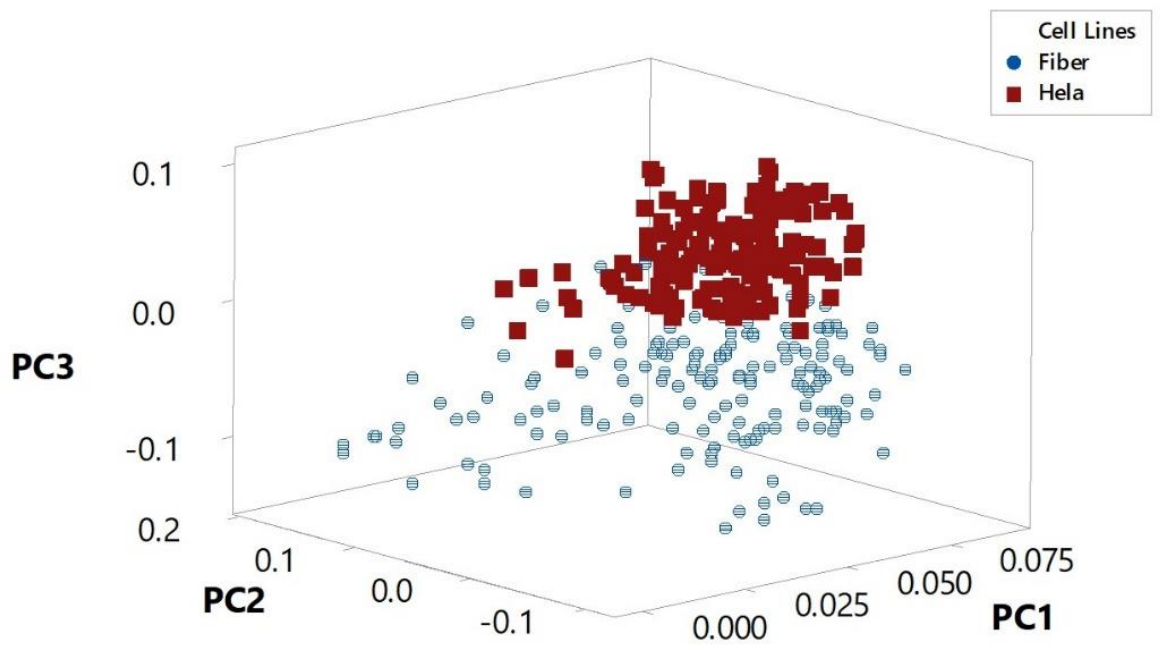

Figure 6.7: Principal component analysis of the data obtained from HeLa and fibroblast cell lines. Scatter plot of HeLa cancer and Fibroblast samples shows differentiation of these cell line based on first three factors of PCA.

In order to demonstrate that SERS-enabled polygonal Si-QP is suitable and efficient for discriminating the HeLa cancer from the control (fibroblast), principle component analysis (PCA) was applied to the collected spectrum. Figure 6.7 shows the 3D scatter plot of PCA based on SERS spectra collected from cancerous HeLa and mammalian fibroblast cells. In this approach, the Raman spectra were first baseline corrected. The multivariate analysis technique was employed by implementation of a linear discriminant algorithm (LDA), to our PCA result as a technique of supervision. The principal components which allowed the best discrimination between HeLa and 
fibroblast in the region $600-1800 \mathrm{~cm}^{-1}$ were Amide V $\left(700-750 \mathrm{~cm}^{-1}\right)$, Amide III $\left(1404 \mathrm{~cm}^{-1}\right)$, phenylalanine (1000-1005 $\left.\mathrm{cm}^{-1}\right)$ and tyrosine $\left(850 \mathrm{~cm}^{-1}\right)$ component. The LDA was used to differentiate the cell types. The PCA-LDA applied to the measured spectra is an objective method to verify if there are significant differences between the HeLa cancer and fibroblast. The PCA allowed us to discriminate between HeLa cancer and fibroblast chosen as a control sample based on the differences existing between the bands that constitute each one of the 289 spectra. These differences between the bands of spectra indicate biochemical differences between samples. LDA identified the two most natural groups separated by blue (fibroblast) and red (HeLa) color shown in Figure 6.7. The sensitivity and specificity of this test method calculated to $86 \%$ and $94 \%$ respectively. Hence, we can use the LDA based on PCA of SERS spectra as a diagnostic method to differentiate cancerous and normal cells with in an acceptable range.

The Raman information taken from HeLa was evaluated in comparison to fibroblast. An abnormal accumulation of esterified cholesterol in lipid droplets was observed in HeLa cell. In a first step, intracellular lipid droplets were visualized by Raman imaging proving that morphologically identical information was obtained. The characteristic $\mathrm{C}=\mathrm{O}$ ester stretching band at $1644 \mathrm{~cm}^{-1}$ was emerging drastically in the course of 24 hours observation in HeLa cells. Whereas it was unidentified in the fibroblast suggesting. On the contrary, spectra of intracellular lipid droplets in HeLa and fibroblast were almost identical in to spectrum of pure cholesteryl bands. Therefore, cholesteryl ester with high percentage in lipid droplets of HeLa was quantified.

the mapping analysis of DNA O-P-O stretch vibrations $\left(783 \mathrm{~cm}^{-1}\right)$ and RNA O-P-O stretch showed the distribution of all nucleic acids in the nucleus for both cell lines. However, an anomalistic increase of the Thymine and Adenine DNA (T, A) were identified at (1176-1220 $\mathrm{cm}^{-}$ $\left.{ }^{1}\right)$ stretching band. This genetic abnormality in DNA might be one of the causes of cells mutation which affect their growth, replication, and ability to survive and invade surrounding tissue. With regards to cancer cells which in many cases show large nucleoli due to their rapid proliferation, statistical analysis of the ratio of nucleic acid to cyto (Figure 6.5 (A)) is in accordance with morphometric analysis of the nucleolus by Raman imaging.

Furthermore, the protein distribution based on the phenylalanine band at (1005 and $\left.1607 \mathrm{~cm}^{-1}\right)$ was found to be significantly high and tracking of the phenylalanine after 6 hours revealed the importance of this protein for proliferation. The noted differences in the distributions of DNA, lipid, and protein could be the basis for future diagnostic applications. 
The differences between HeLa and fibroblast was further examined based on difference spectra and discrimination. T-test analysis displayed significant differences in variety of DNAs and as well as distribution of lipid in both cell lines. Observation of the nuclear envelope of HeLa showed a specific saturated lipid in the membrane. While fibroblast (control cell) possess an unsaturated membrane lipids, their counterparts also contained a high ratio of the nucleic acid to protein concentration.

\subsection{Summary}

This ex vivo study demonstrated the applicability and potential of Raman spectroscopy for detecting cervical precancers with high sensitivity as well specificity. Raman spectroscopy is relatively new tool for non-invasive, real-time and yet promising and powerful analytical technique that can measure the chemical composition of complex biological samples, such as biofluids, cells and tissues for diagnosis of any abnormalities. A Raman spectrum represents a molecular fingerprint of the sample and provides quantitative information regarding its chemical makeup. Biochemical changes in cells and tissues, that may either be caused or are the cause of a disease, can lead to significant changes in the Raman spectra. The potential of Raman spectroscopy arises from its ability to detect such biochemical changes at a molecular level, and therefore, can be used for diagnostics, prognostics or as a tool for evaluating new therapies. However, the current application of Raman spectroscopy is restricted to the use of SERS-active templates where the analyte should be brought to the close vicinity of the template.

Here, we report on the quantum scale probe that is SERS-enabled and can be implemented for in situ live cell Raman spectroscopy. The SERS-enabled polygonal Si-QPs showed a great potential for the investigation of cells, their subcellular structures and for diagnosis. In the present study, we used Raman spectroscopy to examine early-stage (stages 0 and I) HeLa cancer samples ex vivo. The Raman mapping provided molecular information noninvasively and in situ without labeling. Therefore, we performed principal component analysis (PCA) and linear discriminant analysis (LDA) on only the significant wavenumbers assessed by the t-test to have statistically different Raman signal intensities. Linear discriminant analysis (LDA) based on Raman bands found in the 
t-test could predict the tissue types with $86 \%$ sensitivity and $94 \%$ specificity. Comparison between the Raman spectra of cancerous and normal mammalian cell (fibroblast) samples using a PCALDA confirms the significant differences in Amide (III, V), phenylalanine and tyrosine component.

Moreover, a compression of healthy and dead HeLa cell, showed that absence or drastic decline on the concentration of phenylalanine $\left(980-1010 \mathrm{~cm}^{-1}\right)$, tyrosine $\left(1176,1617 \mathrm{~cm}^{-1}\right)$ along with nucleic acids $(\mathrm{G})\left(1320,1420 \mathrm{~cm}^{-1}\right)$ has found to be the indications of cell death. The results indicate the possibility of detecting early-stage HeLa cancer based on molecular information rather than morphological features. Diagnosis with Raman spectroscopy leads to early detection of the pathological changes that take place before the morphological changes and can result in a better outcome of the cancer therapy. Furthermore, it holds potential for the assessment of the effects of chemical treatment. Spectral diagnosis pioneers a new diagnostic technique that is non-invasive, label-free, and less burdensome for the patients. Based on the SERS technique, this could potentially change the way bioassays are performed to improve both the sensitivity and reliability of cancer diagnosis. 


\section{CHAPTER 7}

\section{CONCLUSION}

The Ultrashort Pulsed Laser (USPL) has employed to fabrication/synthesis the nanomaterials in this thesis. As mentioned before precisely tuning the Laser parameters can result in complete different physicochemical behavior of the synthesized nanomaterial. Hence, this chapter summarizes the influential Laser parameters by which distinctive nanomaterials with unique properties was synthesized.

\subsection{Functionalization of stress components}

The initial study was dedicated to cell matter interaction and residual stress was induced onto the substrate (silicon wafer) as a stimuli to modulate cellular behavior. In order to accurately tune the functionality of the induced residual stress (IRS), the silicon chip was exposed to the irradiation from an Ultrashort Pulsed Laser (USPL). The combination of shockwave formations and intense thermal expansion generated by the laser irradiation induces crystal distortion. The resulting mismatch of distorted crystal orientation and virgin crystals causes residual stress. The functionality of the stress component was controlled by varying repetition rate (rep.rate) of the laser. The repetition rate determines the level of stress of the functionalized stress component, and it tunes the degree of biocompatibility of the silicon chip (bio-template). The difference in temperature between face and subsurface of the silicon bio-template, caused by rapid heat dissipation, creates intricate crystal distortions. This changes the crystal orientation of the IRS zone from inherent (100) to (211) and a different order of crystallinity. In particular, amplifying the repetition rate from $4 \mathrm{MHz}$ to $26 \mathrm{MHz}$ causes extensive accumulation of heat on the exposure zone - owing to maximizing laser-matter interaction time, more thermal and shockwave energy is transferred to the substrate. Therefore, incremental heat generation intensifies thermal stress, resulting in the formation of the laser induced residual stress zone. 


\subsection{Transition from crystalline to amorphous structure}

In order to generate the nanomaterials by utilizing laser, the amount of energy transferred to the substrate (silicon chip) has to be above ablation threshold of silicon. The absorption of the photon energy delivered by Laser irradiation onto the substrate initiates the ablation process. The Laser ablation causes an ionization and the ionized species form a Laser plume. The precise control of the ionization level during synthesis enabled us to tune the crystallinity and yield of the synthesized nanomaterials. Hence, The Silicon Nano Webs (SNWs) were synthesized by single-step femtosecond laser processing of silicon ( $\mathrm{Si}$ ) chips in atmospheric conditions. A computerized Galvano scanner laser enabled precise 2D (X-Y) movement of the laser beam on the Si chip surface. Therefore, pre-designed patterns transferred onto the surface of the Si samples. The laser processing of the Si chips at megahertz $(\mathrm{MHz})$ pulse repetition rates triggers surface ablation, evaporation, and the formation of the plume, which is composed of ionized $\mathrm{Si}$ and air molecules. Expansion of the plume in the background air causes condensation as a result of temperature dissipation, leading to the formation of the nucleus and growth of self-assembled silica nanoclusters. The nanoclusters after plume condensation form a deposition of accumulated nanowebs on the Si samples. The packing index of synthesized silica nanowebs is a function of self-assembled nanoparticle size variation during the ionization process. The most influential laser parameters by which these attributes have been governed are laser repetition rate and pulse duration. Increasing the increment of the repetition rate from $4 \mathrm{MHz}$ to $26 \mathrm{MHz}$ decreases the average nanoparticle size. Furthermore, the increment of the repetition rate has a direct relationship with the yield of the synthesized nanoweb. Moreover, the average area of vacuities of the nanoweb, measured using image analysis software, was defined as a packing index of synthesized nanomaterials. Reduction of nanoparticle size along with the increment of yield materials contributed to closely pack synthesized nanostructures with an average area of 3.1, 2.5 , and $0.9 \mathrm{~nm}^{2}$, respectively, for 4,12 , and $26 \mathrm{MHz}$. Therefore, ionization energy governed by laser fluence has an analogous influence on the packing index of the condensed silica nanoweb. Additionally, prolonging the laser pulse from 214 fs to 1428 fs showed the predominance of the amorphous phase of the silica structure, in which the ionized molecular fragments were desorbed, believed to be caused of amorphization. Atmospheric ablation lead to chemical phase transformation through which $\mathrm{Si}-\mathrm{O}$ bonding occurs. The use of ultra-short 
pulsed laser (USPL) has provided the advantages of single-step processing of a silica nanoweb through which precisely tuning the laser parameters controls the particle size as well as direct amorphization.

\subsection{Tunability of alteration of crystallinity}

This study shows that the USPL is capable to overcome the ionization threshold of the silicon substrate by inducing sufficient photon energy. The laser irradiation of the substrate causes absorption of one or more photons by electrons in atoms of the sample (a single-crystal silicon wafer) which ultimately results in spatial electron separation of the excited atom or ion species. The accumulated energy of multiphoton absorption exceeds the ionization potential of the silicon and hence leads to atomic ionization. Therefore, the kinetics of the self-assembly process were initiated by multi-photoionization. The Photoluminescence Silicon Nano probes (PLSN-probes) created based on kinetic self-assembly activated by multiphoton ionization. Utilization of USPL able a systematically transform a non-luminescent bulk single-crystal silicon wafer into multiplex photoluminescent nano-probes with tunability via synthesized crystal orientations. The experiment showed that multi-photoionization can be governed by precisely tuning the laser fluence. The numbers of crystal planes by which higher absorption and subsequently the FL emission achieved, was controlled by precisely tuning the laser fluence. When a sufficiently higher laser fluence interacts with the substrate, it results in the formation of a 3D self-assembled structure consisting of multiplanar crystal Si (polyhedral). Likewise, a lower laser fluence resulted in fewer crystal planes (polyhedron). The synthesis of the PLSN-probes can be precisely tuned by regulating the laser parameters. While the laser pulse duration and laser power were kept constant at $214 \mathrm{fs}$ and $16 \mathrm{~W}$, respectively, high, intermediate and low laser fluences were achieved at a 4-, 12- and 26$\mathrm{MHz}$ repetition rate, respectively. The transition from a single (polyhedron) to multiplanar (polyhedral) silicon crystal structure yields a higher absorption and hence a superior FL throughput. The use of USPL-assisted ionization to alter the crystallinity of the synthesized Silicon Nano particles (Si-NPs) under inert atmospheric conditions resulted in impurity-free and wellcharacterized 3D structures. 


\subsection{Conclusions}

In this thesis, the Si-based nanomaterials synthesized by ultra-short pulsed laser in femtosecond region that exhibits therapeutic and diagnostic applications are presented. The unique structure, shape, phases, size, and morphology has been generated by precisely controlling the laser parameter such as laser power, repetition rate, laser pulse width, scanning speed, and atmospheric conditions. Wide range of physical/chemical properties have been extracted from the synthesized nanostructures/particles such as single-multiple crystallinity, crystalline-amorphous structure, active-passive chemistry, and optical attribution of multiplex luminesce.

Above mentioned properties have been proposed and exploited in different application in respect to the scope of this study. However, they can potentially be used in other areas such as microelectronics, MEMS, lab-on-a-chip, and optics.

In respect to therapeutic application, simultaneous benefits of cell attraction and cell-selective treatment lead to high efficiency of cancer therapy and at the same time proliferative tissue generation. This unique property of cell selectively was applied to next objective of diagnosis.

The synthesize of multiplex photoluminescence nanomaterial that is biocompatible can benefit the cell imaging and overcome the current hindrance of conventional organic dyes. Here it is reported that different cell organelles can be identified in specific excitation wavelength. Moreover, the funding of the previous study implemented to diagnose HeLa cancer cells. Additionally, for the first time 3D intercellular imaging has made possible.

Cancer diagnosis is crucially important since early diagnosis can save lives. Therefore, the capability of silicon based nanomaterials for Raman spectroscopy that has been established in our research lab was exploited for cancer diagnosis. However, Silicon nanomaterials have been used for Raman sensing. They are restricted to template for detection. Here, the particles in quantum size was enabled to be used individually. This attribution (SERS-enabled) was given to so call $\mathrm{Si}$ Quantum probes by transforming spherical to polygonal morphology. The result revealed the fingerprint of HeLa and fibroblast cells by which cell differentiation and diagnosis was made possible. 
Another achievement of this study is to introduce that cells (mammalian and cancer) are sensitive and able to perceive the residual stress. This funding suggests a new element to communicate with biological cells by which their response in this case was collective migration to stress-free zone.

The findings and achievement of this thesis can be summarized as following;

- New concept of functionalized stress component to regulate cellular behaviors such as collective cell migration, tunable directionality. Moreover, the minimum required stress for cells to response base on the cell type has identified.

- The benefits of commonly used ECM and nanoparticles in biology have been concurrently combined and exploited for selective therapeutic purposes. This platform exhibited proliferative and induction of intensified apoptotic pathway to fibroblast and HeLa cells respectively. It realized that the amorphized silicon nanostructures can be attractive for both cells to adhere. However, kills cancerous HeLa cells through programed cell death (apoptosis).

- The unique colloid -liked silicon particles in quantum size range with ability to disintegrate and internalize into a cell of interest has shown a capability to be replaced by conventional organic dyes. These agents have the advantage of being used for bioimaging of different part of a cell base on permeability preferences of organelles membrane. Additionally, distinctive response of HeLa and fibroblast to these bioimaging agents led to diagnosis application of them.

- The last but not least, is enabling a quantum sized silicon particle to act as a SERS-active for Raman spectroscopy. The facial polytonality of the synthesized probes has enabled localized surface plasmon along with an affinity for $\mathrm{C}-\mathrm{H}$ function group bonding, by which each individual particle functions as a receptor for biochemical sensing. The cell viability assessment have proven that these probes can be used for live cell sensing. The revealed fingerprint of HeLa and fibroblast resulted in cell differentiation, and cancer diagnosis. 


\subsection{Future research and recommendations}

With the development of these Si-based nanostructures/nanoparticles that can exhibit multiplex photoluminescence and SERS enhancement, the possibility of further developing of dual functional bioimaging-diagnosis probe can be envisioned. Moreover, some preliminary results suggest that the proposed cancer diagnostic method can be utilized for different cancer cell lines in this case breast cancer was examined and showed promising outcomes. The other application of Raman spectroscopy in biomedicine and biotechnology can be detection of bacterial infection. Preliminarily results demonstrated the feasibility of this sensing method for E. coli detection. 


\section{APPENDIX}

\section{Appendix 1 Influence of suspended amorphous nanostructures}

The influence of suspended amorphous nanostructures (freestanding SNWs) on HeLa cell after 24h was observed as shown in SEM and FM images.
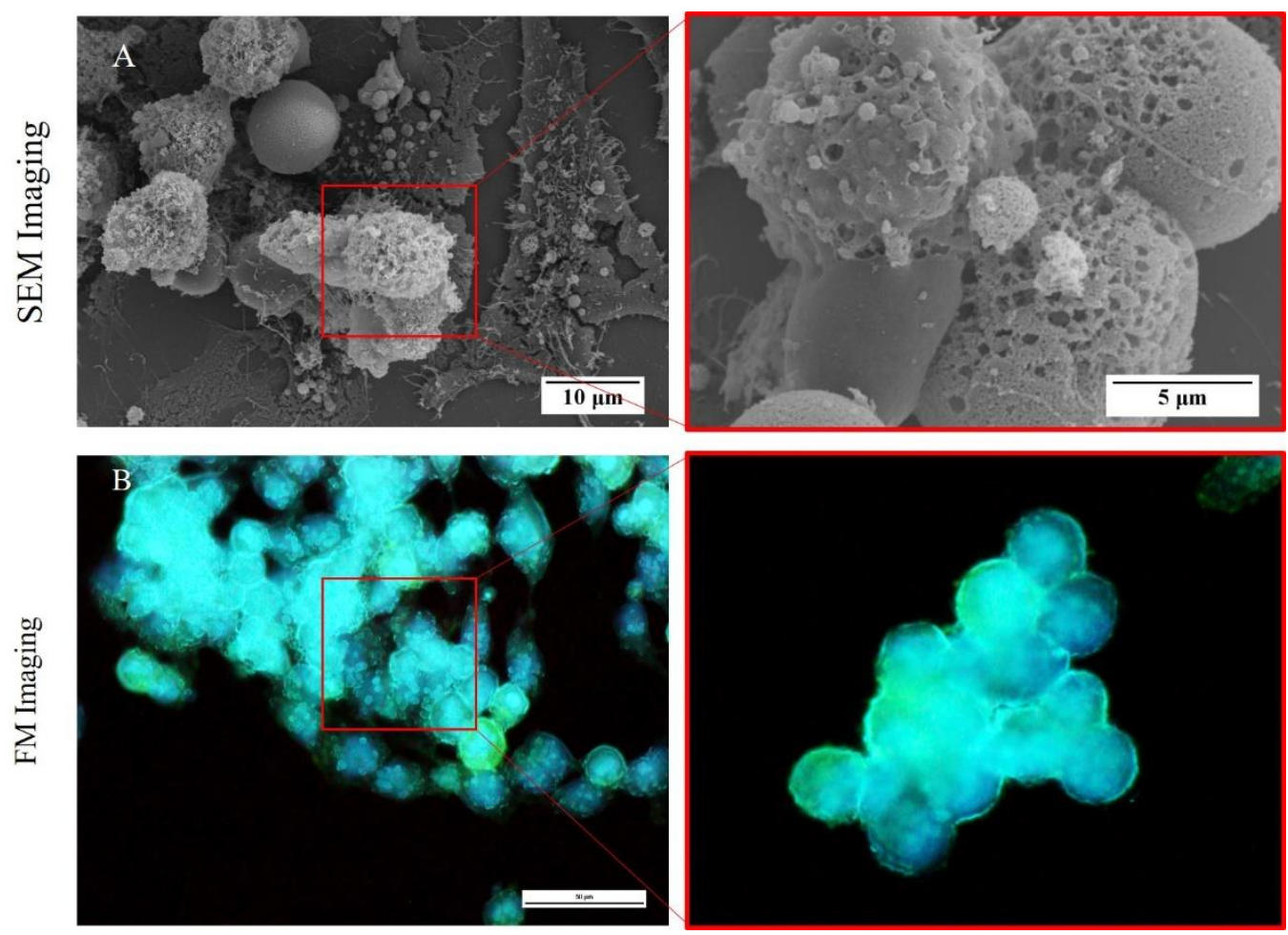

Figure S1: SEM (A) images show the HeLa cell interaction with suspended Amo-SNSs. Cell roundness is the evidence of cell death induced by endocytosis. The zoom in image shows the porous cytoskeleton of cell that represents the final stage of apoptosis pathway. FM images (B) stained in blue and green distinguish the cytoskeleton and the nucleolus respectively. The penetration of SNWs and their accumulation in the cell body evidenced the cause of cell death. 


\section{Appendix 2 Characterization of the hybrid SWNs}
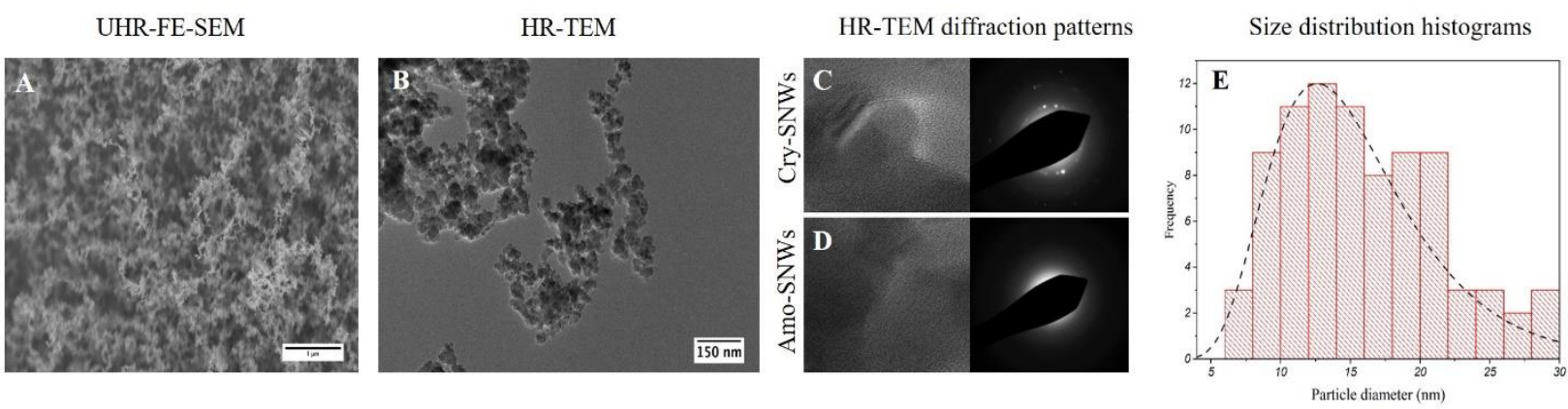

Figure S2: Characterization of the hybrid SWNs evidences the simultaneous presence of crystalline and amorphous orientation. UHR-FE-SEM micrograph of the Hybrid SNWs shows the morphological structure of as-synthesized SNWs (A). HR-TEM image used for particle size measurement (B) shows self-assembled SNWs. Diffraction patterns (C-D) taken by HRTEM evidence the formation of Cry- and Amo-SWNs. Size distribution histogram (E) show an average size of $12 \pm 0.5 \mathrm{~nm}$ (Statistical analysis done over 1000 particles count).

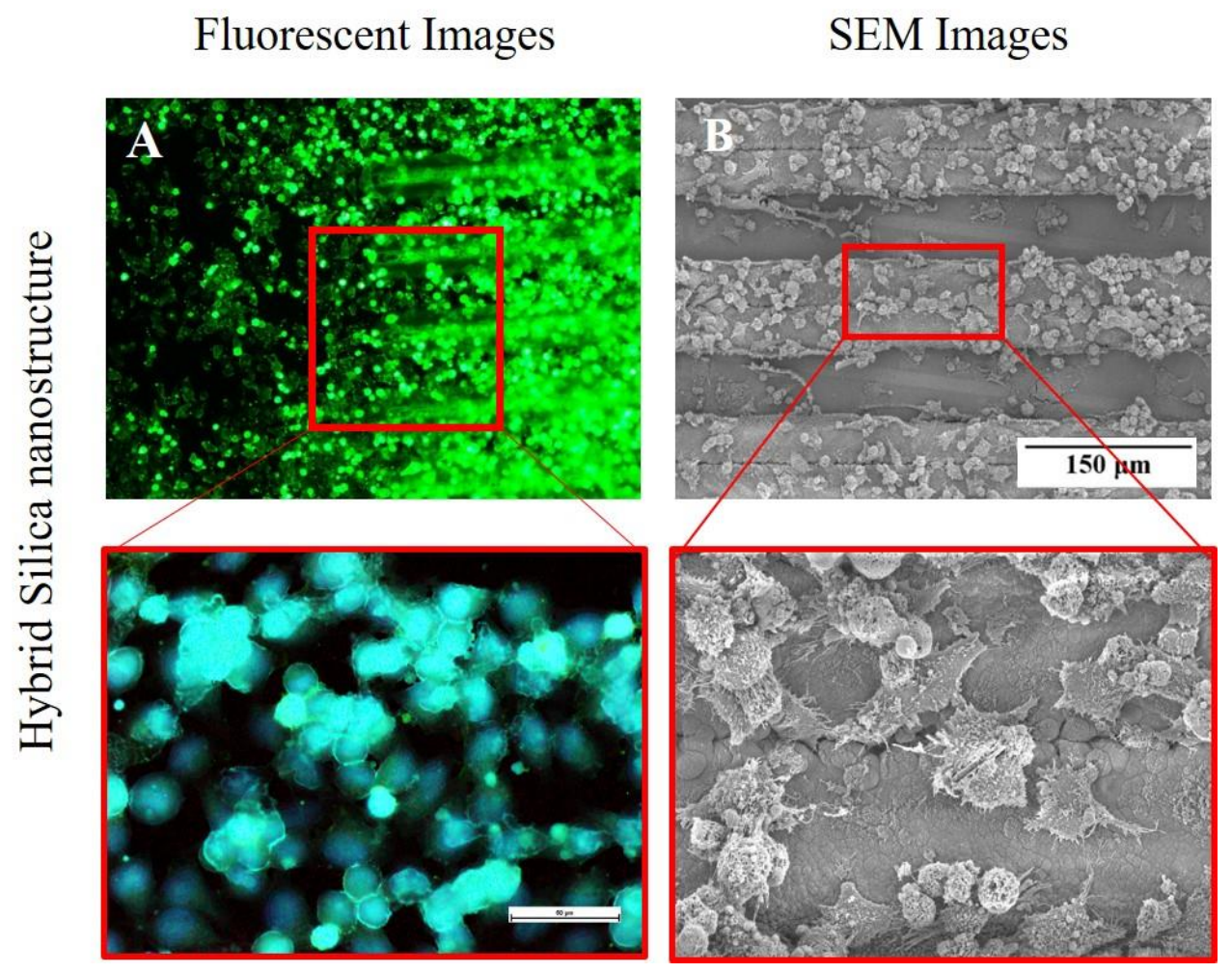

Figure S3: Hybrid Silica Nano-web composes of crystalline and amorphous was synthesized at intermediate laser pulse width of 714 fs. The FM and SEM images (A and B) showed that efficiency of this nanostructure to attract cells has remained same, regardless of the crystallinity of SNWs. However, HeLa cells have shown rounded and ruptured cytoskeleton. The number of dead cells is not comparable with Amo-SNWs. 


\section{Appendix 3 Biomolecular fingerprint}

\begin{tabular}{|l|l|}
\hline Lipids (CH2 twist) & 1301 \\
\hline Lipids (C=O ester) & 1736 \\
\hline Lipids (C=C str) & $1680-1655$ \\
\hline Lipids (CN+(CH3)3 str) & 717 \\
\hline Lipid (R) & 2855 \\
\hline Lipid (v (CH2) ) & 2845 \\
\hline Lipids (C-C-N+ sym str); Carbohydrates (C-O-C ring) & 877 \\
\hline C-H vibrations (Peaks of lipids; Hydrocarbon chains) & 1301 \\
\hline C=C stretching (Peaks of lipids; Hydrocarbon chains) & 1660 \\
\hline (Peaks of lipids; Hydrocarbon chains) & 1449 \\
\hline $\begin{array}{l}\text { Lipid \& Protein Guanine (DNA and/or RNA), CH def. in } \\
\text { proteins and carbohydrates }\end{array}$ & 1655,1340 \\
\hline Lipid \& Protein ( CH2 vibration ) & 1446 \\
\hline Proteins (C-C twist Tyr) & 645 \\
\hline protein (B) & 1689 \\
\hline $\begin{array}{l}\text { Proteins (C-C twist Phe) C-C twisting mode of } \\
\text { phenylalanine (proteins) }\end{array}$ & 621 \\
\hline $\begin{array}{l}\text { Proteins (Ring br Tyr) Ring breathing in tyrosine and } \\
\text { proline (proteins) }\end{array}$ & 854 \\
\hline Proteins (Ring breath Trp) & 760 \\
\hline Proteins (C-C6H5, Phe,Trp) & 1209 \\
\hline Proteins (C-H bend, Tyr) & 1176 \\
\hline Proteins (C=C Tyr, Trp) & 1617 \\
\hline Proteins (C=C Phe, Tyr) & 1607 \\
\hline Protein beta sheet & 1686 \\
\hline Proteins (HSA) & 1005,756 \\
\hline Proteins (C-H in-plane Phe) & 1033 \\
\hline Proteins (Sym. Ring br Phe) & 1005 \\
\hline Proteins (C-C BK str. $\beta$-sheet); Lipids (=CH Bend) & 980 \\
\hline $\begin{array}{l}\text { Proteins (C-C BK str. } \alpha \text {-sheet); Carbohydrates (C-O-C } \\
\text { glycos) }\end{array}$ & 937 \\
\hline Protein (phenylalanine) & $1004,1000,1002-1005$ \\
\hline Amide VI Symmetric breathing of tryptophan (protein) & $630-750$ \\
\hline Amide V Symmetric breathing of tryptophan (protein) & $700-750$ \\
\hline Amide III & $1230-1300,1200-1300,1218 / 1222,1295$, \\
\hline Amide II & $1250,1254-1255$ \\
\hline $\begin{array}{l}\text { Amide I Amide I (C=O stretching, C-N stretching, and } \\
\text { N-H bending, proteins) }\end{array}$ & $\sim 1550$ \\
\hline Amide I; Protein & $1655,1600-1700 ~ 1620-1700,1660-1670$, \\
\hline Proteins(C-N str.); Carbohydrates (C-O str) & $1650-1655,1660$ \\
\hline Protein \& Unsaturated lipid (Amide I v (C=O); v (C=C)) & 1128 \\
\hline Nucleic Acids (A) & 7655 \\
\hline
\end{tabular}




\begin{tabular}{|c|c|}
\hline Nucleic Acids $(T, G)$ & 667 \\
\hline Nucleic Acids (G,A) & 1578 \\
\hline Nucleic Acids (O-P-O asym str.); Proteins (Ring br Tyr) & 828 \\
\hline Nucleic Acids (O-P-O asym str. RNA) & 811 \\
\hline Nucleic Acids (O-P-O asym str. DNA) & 788 \\
\hline $\begin{array}{l}\text { Nucleic Acids (U,C,T ring br) Uracil, thymine, cytosine } \\
\text { (ring-breathing modes in the DNA and/or RNA) }\end{array}$ & 782 \\
\hline $\begin{array}{l}\text { nucleic acids of DNA Thymine, guanine (DNA and/or } \\
\text { RNA) Adenine, guanine (DNA and/or RNA); } \mathrm{C}=\mathrm{C} \\
\text { bending mode of phenylalanine }\end{array}$ & $670,830,1375,1580$ \\
\hline nucleic acids of RNA & 815 \\
\hline C5-O-P-O-C3 phosphodiester bonds in DNA & 788 \\
\hline C5-O-P-O-C3 phosphodiester bonds in RNA & 813 \\
\hline $\begin{array}{l}\text { DNA Uracil, thymine, cytosine (ring-breathing modes in } \\
\text { the DNA and/or RNA) }\end{array}$ & 782,788 \\
\hline $\begin{array}{l}\text { RNA Uracil, thymine, cytosine (ring-breathing modes in } \\
\text { the DNA and/or RNA) }\end{array}$ & 782,813 \\
\hline $\mathrm{U}, \mathrm{C}, \mathrm{T}$ ring br (RNA) & 780 \\
\hline Ribose phosphate (DNA bkb) & 895 \\
\hline $\mathrm{C}, \mathrm{T}, \mathrm{DNA}$ bkb (O-P-O str) & 792 \\
\hline O-P-O RNA & $818 / 805$ \\
\hline $\begin{array}{l}\text { Nucleic Acids (PO2 str.); Lipids (Chain C-C str.); } \\
\text { Carbohydrates (C-O, C-C str) }\end{array}$ & $1060-1095$ \\
\hline $\begin{array}{l}\text { Nucleic acids (Symmetirc phosphodiester stretch; Ring } \\
\text { breathing modes of pyrimidine bases) }\end{array}$ & $783-790$ \\
\hline $\begin{array}{l}\text { Nucleic acids \& lipid (Symmey dioxy-stretch of the } \\
\text { phosphate backbone v (C-C)) }\end{array}$ & 1090-1010 \\
\hline $\begin{array}{l}\text { Nucleic Acids (G,A, CH def); Proteins (C-H); Lipids } \\
\text { (CH def); Carbohydrates (CH def) }\end{array}$ & $1480-1420$ \\
\hline $\begin{array}{l}\text { Nucleic Acids (A,G); Proteins (C-H); Carbohydrates } \\
\text { (CH def) }\end{array}$ & 1342 \\
\hline Nucleic Acids (G); Proteins (C-H) & 1320 \\
\hline $\begin{array}{l}\text { Nucleic Acids (T,A); Proteins (Amide III); Lipids (=CH } \\
\text { bend) Amide III (C-N stretching, N-H bending, } \\
\text { proteins), PO2 asymmetric stretching (DNA and/or } \\
\text { RNA) }\end{array}$ & $1220-12841220-1280$ \\
\hline Pyrimidine bases; Nucleic acids & $785-788$ \\
\hline $\begin{array}{l}\text { A,T,C Amide II (N-H bending, C-N stretching, } \\
\text { proteins); adenine, guanine (DNA and/or RNA) }\end{array}$ & 1485 \\
\hline $\mathrm{A}, \mathrm{T}, \mathrm{G}$ & 1475 \\
\hline $\begin{array}{l}\text { A, G Adenine, guanine (DNA and/or RNA); } \mathrm{C}=\mathrm{C} \\
\text { bending mode of phenylalanine }\end{array}$ & $1432,1480,1580$ \\
\hline Ademine & 725 \\
\hline $\begin{array}{l}\beta \text {-carotene PLGA nanoparticles CH2 and } \\
\text { CH3 symmetric stretching (lipids) }\end{array}$ & $1002,1450,1657,2940$ \\
\hline
\end{tabular}




\begin{tabular}{|c|c|}
\hline$\beta$-carotene and lipids in superposition & $1004,1153,1520$ \\
\hline Biological molecules have virabtions & $\sim 600-3000$ \\
\hline $\begin{array}{l}\text { Cytochrome c } \mathrm{C}-\mathrm{N} \text { stretching in proteins; } \mathrm{C}-\mathrm{O} \\
\text { stretching in carbohydrates }\end{array}$ & 753,1130 \\
\hline Cytochrome & $605,753,1314,1585$ \\
\hline Cytosine, Thymine & 783 \\
\hline C4-C5=C6 (cytosine), $\mathrm{C} 2=\mathrm{O}$ (cyrosine) & 820 \\
\hline C6-H, C4-NH2, C5=C6 (cytosine) & 1360 \\
\hline C2-N3 (cyrosine), C4-NH2 (cytosine) & 1275 \\
\hline C6-H, N1-H, C5-H (cytosine) & 1252 \\
\hline $\mathrm{CH} 2 \mathrm{def}$ & 1446 \\
\hline C5-H, C2-N3-C4-C5 (cytosine) & 791 \\
\hline Cytosine & 780 \\
\hline $\mathrm{C} 2=\mathrm{O}$ (cytosine) & 1694 \\
\hline C5-H (cytosine), $\mathrm{NH} 2$ & 700 \\
\hline $\begin{array}{l}\mathrm{C}-\mathrm{C} \text { stretching Adenine, guanine (DNA and/or RNA); } \\
\mathrm{C}=\mathrm{C} \text { bending mode of phenylalanine }\end{array}$ & $964,1580,828$ \\
\hline C-C skeletal & 952 \\
\hline C-N str. Phe (C-H in-plane) & 1025 \\
\hline $\mathrm{C}, \mathrm{G}, \mathrm{A}$ & $1188 / 1185 ; 1534$ \\
\hline $\mathrm{CH} 2$ ring str, $\mathrm{CH} 3$ twist, $\mathrm{A}$ & 1312 \\
\hline C-C stretch [C,G,C (me)] & 1100 \\
\hline C4-C5 (cytosine) & 1011 \\
\hline $\mathrm{C}-\mathrm{H}, \mathrm{N} 3-\mathrm{C} 4-\mathrm{C} 5=\mathrm{C} 6$ (cyrosine) & 992 \\
\hline $\begin{array}{l}\text { CG(C3, C4,C5 me) Symmetric breathing of tryptophan } \\
\text { (protein) }\end{array}$ & $\begin{array}{l}\text { 783, 877, 1101, 647, 681, 1361, 750; 784, } \\
\text { 752, 1523, 1356, 877; }\end{array}$ \\
\hline $\begin{array}{l}\text { CG(C8 me) Uracil, thymine, cytosine (ring-breathing } \\
\text { modes in the DNA and/or RNA) }\end{array}$ & $\begin{array}{l}753,680,1492,1253 ; 681,782,1257,1107, \\
1471 ;\end{array}$ \\
\hline Cyrosine, Guanine & $1184-1180$ \\
\hline $\begin{array}{l}\text { C5-H (cytosine) } \mathrm{C}-\mathrm{C} \text { and } \mathrm{C}-\mathrm{N} \text { stretching of proteins } \\
\text { and/or lipids }\end{array}$ & 1155 \\
\hline C-C/C-N str. & 1158 \\
\hline $\mathrm{CH} 2, \mathrm{CH} 3$; Protein lipids & $1425-1475$ \\
\hline $\mathrm{CH} 3, \mathrm{CH} 2, \mathrm{CH}$; Lipids, proteins, other & $2800-3020$ \\
\hline cyto c (G) Symmetric breathing of tryptophan (protein) & 750 \\
\hline cyto c $(\mathrm{B})$ & 747 \\
\hline C-H vibrations (Amino acids containing Phenyl groups) & 1449 \\
\hline $\begin{array}{l}\text { Carbohydrates; C-O-C vibrations of the glycosidic bonds } \\
\& \text { sugar rings }\end{array}$ & $800-1100$ \\
\hline Deuterated arachidonic acid (AA-d8), F & $2200-2280$ \\
\hline $\begin{array}{l}\text { Groups of phospholipids; C-C-N+ symmetric stretching } \\
\text { in phosphatidylcholine }\end{array}$ & 719 \\
\hline Glucose \& membrane lipids & 1125 \\
\hline N1-H (cyrosine), NH2 & 599 \\
\hline N1-H (cyrosine), C4-C5 (cytosine), CH & 1463 \\
\hline
\end{tabular}




\begin{tabular}{|l|l|}
\hline NH2, C2-N3-C4, N-C,C=O (cytosine) & 1108 \\
\hline NH2 bending, C4- NH2 (cytosine) & 1613 \\
\hline NH bending, C4-N3 (cyrosine) & 1534 \\
\hline O-P-O; Nucleic acid backnone; phospholipids & $1092-1095$ \\
\hline Phenylalanine (Amino acids containing Phenyl groups) & 1005 \\
\hline Polystyrene, E & 1000 \\
\hline Phosphoric acid & 800 \\
\hline Phosphate backbone & $1090-1087,938$ \\
\hline Pyrimidine rings [C,C(me)] & 1243 \\
\hline Pyrimidine rings (cytosine) & 1579 \\
\hline T,C & 785 \\
\hline $\begin{array}{l}\text { Tyr (C-C twsit) C-C twisting mode of tyrosine and } \\
\text { phenylalanine }\end{array}$ & 642 \\
\hline T,G Thymine, guanine (DNA and/or RNA) & 670 \\
\hline Tyr, A & $736 / 732$ \\
\hline Try, ribose phosphate (DNA) & $834 / 832$ \\
\hline $\begin{array}{l}\text { tyrosine (Amino acids containing Phenyl groups) Ring } \\
\text { breathing in tyrosine and proline (proteins) }\end{array}$ & 854 \\
\hline trytophan (Amino acids containing Phenyl groups) & 760 \\
\hline $\begin{array}{l}\text { thymine, cytosine and uracil Uracil, thymine, cytosine } \\
\text { (ring-breathing modes in the DNA and/or RNA) }\end{array}$ & 782 \\
\hline VsPO2- PO2 symmetric stretching (DNA and/or RNA) & 1080 \\
\hline $\begin{array}{l}\text { VasPO2- Amide III (C-N stretching, N-H bending, } \\
\text { proteins), PO2 asymmetric stretching (DNA and/or } \\
\text { RNA) }\end{array}$ & 1220 \\
\hline C-N stretching in adenine and lipids & 720 \\
\hline PO2 stretching in DNA, tyrosine & 827 \\
\hline C-C stretching mode of proline and valine & 935 \\
\hline $\begin{array}{l}\text { C-H bending mode in phenylalanine, C-N stretching in } \\
\text { proteins }\end{array}$ & 1030 \\
\hline C-O stretching & 1085 \\
\hline $\begin{array}{l}\text { C-H in plane-bending mode of tyrosine and } \\
\text { phenylalanine; cytosine, guanine }\end{array}$ & 1175 \\
\hline C-C6H5 stretching mode in tryptophan, phenylalanine; & 1208 \\
\hline CH3 and/or CH2 twisting mode of collagen and lipid & 1311 \\
\hline CH (CH2) bending mode in proteins and lipids & $1430-1460$ \\
\hline C=C phenylalanine, tyrosine, and tryptophan & 1615 \\
\hline CH2 symmetric stretching (lipids) & 2890 \\
\hline CH3 symmetric stretching (lipids) & 2980 \\
\hline
\end{tabular}




\section{REFERENCES}

3-1. Li, Y. et al. Engineering cell alignment in vitro. Biotechnology Advances 32, 347-365 (2014).

3-2. Rhee, S. Fibroblasts in three dimensional matrices: cell migration and matrix remodeling. Exp Mol Med 41, 858-865 (2009).

3-3. Charras, G. \& Sahai, E. Physical influences of the extracellular environment on cell migration. Nat Rev Mol Cell Biol 15, 813-824 (2014).

3-4. Legant, W.R. et al. Measurement of mechanical tractions exerted by cells in three-dimensional matrices. Nat Meth 7, 969-971 (2010).

3-5. Tan, J.L. et al. Cells lying on a bed of microneedles: An approach to isolate mechanical force. Proceedings of the National Academy of Sciences 100, 1484-1489 (2003).

3-6. Chooi, W.H. \& Chan, B.P. Compression loading-induced stress responses in intervertebral disc cells encapsulated in 3D collagen constructs. Scientific Reports 6, 26449 (2016).

3-7. Molino, D. et al. On-Chip Quantitative Measurement of Mechanical Stresses During Cell Migration with Emulsion Droplets. Scientific Reports 6, 29113 (2016).

3-8. Hakkinen, K.M., Harunaga, J.S., Doyle, A.D. \& Yamada, K.M. Direct Comparisons of the Morphology, Migration, Cell Adhesions, and Actin Cytoskeleton of Fibroblasts in Four Different Three-Dimensional Extracellular Matrices. Tissue Engineering. Part A 17, 713-724 (2011).

3-9. du Roure, O. et al. Force mapping in epithelial cell migration. Proceedings of the National Academy of Sciences of the United States of America 102, 2390-2395 (2005).

3-10. Gribova, V., Crouzier, T. \& Picart, C. A material's point of view on recent developments of polymeric biomaterials: control of mechanical and biochemical properties. Journal of Materials Chemistry 21, 14354-14366 (2011).

3-11. Li, S.S. et al. Fabrication of gelatin nanopatterns for cell culture studies. Microelectronic Engineering 110, 70-74 (2013). 
3-12. Bettinger, C.J., Langer, R. \& Borenstein, J.T. Engineering Substrate Topography at the Micro- and Nanoscale to Control Cell Function. Angewandte Chemie International Edition 48, 5406-5415 (2009).

3-13. Gao, H. et al. Effective Spatial Separation of PC12 and NIH3T3 Cells by the Microgrooved Surface of Biocompatible Polymer Substrates. Langmuir 31, 6797-6806 (2015).

3-14. Jeon, H. et al. Directing cell migration and organization via nanocrater-patterned cellrepellent interfaces. Nat Mater 14, 918-923 (2015).

3-15. Richert, L. et al. Surface Nanopatterning to Control Cell Growth. Advanced Materials 20, 1488-1492 (2008).

3-16. Gan, J. et al. Fabrication of cell pattern on poly(dimethylsiloxane) by vacuum ultraviolet lithography. Colloids and Surfaces B: Biointerfaces 76, 381-385 (2010).

3-17. Zhou, X., Hu, J., Li, J., Shi, J. \& Chen, Y. Patterning of Two-Level Topographic Cues for Observation of Competitive Guidance of Cell Alignment. ACS Applied Materials \& Interfaces 4, 3888-3892 (2012).

3-18. Dalby, M.J. et al. The control of human mesenchymal cell differentiation using nanoscale symmetry and disorder. Nat Mater 6, 997-1003 (2007).

3-19. Jeon, H. et al. Chemical Patterning of Ultrathin Polymer Films by Direct-Write Multiphoton Lithography. Journal of the American Chemical Society 133, 6138-6141 (2011).

3-20. Jeon, H., Hidai, H., Hwang, D.J., Healy, K.E. \& Grigoropoulos, C.P. The effect of micronscale anisotropic cross patterns on fibroblast migration. Biomaterials 31, 4286-4295 (2010).

3-21. Premnath, P., Venkatakrishnan, K. \& Tan, B. Programming cancer through phasefunctionalized silicon based biomaterials. Sci. Rep. 5 (2015).

3-22. Premnath, P., Tan, B. \& Venkatakrishnan, K. Direct Patterning of Free Standing Three Dimensional Silicon Nanofibrous Network to Facilitate Multi-Dimensional Growth of Fibroblasts and Osteoblasts. Journal of Biomedical Nanotechnology 9, 1875-1881 (2013). 
3-23. Premnath, P., Tan, B. \& Venkatakrishnan, K. Engineering functionalized multi-phased silicon/silicon oxide nano-biomaterials to passivate the aggressive proliferation of cancer. Scientific Reports 5, 12141 (2015).

3-24. Bhuyan, M.K., Rodriguez-Devora, J.I., Fraser, K. \& Tseng, T.-L.B. Silicon substrate as a novel cell culture device for myoblast cells. Journal of Biomedical Science 21, 47-47 (2014).

3-25. Fan, Y.W. et al. Adhesion of neural cells on silicon wafer with nano-topographic surface. Applied Surface Science 187, 313-318 (2002).

3-26. Voskerician, G. et al. Biocompatibility and biofouling of MEMS drug delivery devices. Biomaterials 24, 1959-1967 (2003).

3-27. Wu, B., Tao, S. \& Lei, S. Numerical modeling of laser shock peening with femtosecond laser pulses and comparisons to experiments. Applied Surface Science 256, 4376-4382 (2010).

3-28. Tien, A.-C., Backus, S., Kapteyn, H., Murnane, M. \& Mourou, G. Short-Pulse Laser Damage in Transparent Materials as a Function of Pulse Duration. Physical Review Letters 82, 3883-3886 (1999).

3-29. Gujba, A. \& Medraj, M. Laser Peening Process and Its Impact on Materials Properties in Comparison with Shot Peening and Ultrasonic Impact Peening. Materials 7, 7925 (2014).

3-30. Rubio-González, C. et al. Effect of an absorbent overlay on the residual stress field induced by laser shock processing on aluminum samples. Applied Surface Science 252, 6201-6205 (2006).

3-31. Cheng, G.J. \& Shehadeh, M.A. Dislocation behavior in silicon crystal induced by laser shock peening: A multiscale simulation approach. Scripta Materialia 53, 1013-1018 (2005).

3-32. Zheng, X., Li, J. \& Zhou, Y. X-ray diffraction measurement of residual stress in PZT thin films prepared by pulsed laser deposition. Acta Materialia 52, 3313-3322 (2004).

3-33. Alhomoudi, I.A. \& Newaz, G. Residual stresses and Raman shift relation in anatase $\mathrm{TiO} 2$ thin film. Thin Solid Films 517, 4372-4378 (2009).

3-34. Colomban, P. Analysis of Strain and Stress in Ceramic, Polymer and Metal Matrix Composites by Raman Spectroscopy. Advanced Engineering Materials 4, 535-542 (2002). 
3-35. De Wolf, I., Chen, J., Rasras, M., van Spengen, W.M. \& Simons, V., Vol. 3897 239-252 (1999).

3-36. Wolf, I.D. Stress measurements in Si microelectronics devices using Raman spectroscopy. Journal of Raman Spectroscopy 30, 877-883 (1999).

3-37. Costa, D.O. et al. Control of Surface Topography in Biomimetic Calcium Phosphate Coatings. Langmuir 28, 3871-3880 (2012).

3-38. Ranella, A., Barberoglou, M., Bakogianni, S., Fotakis, C. \& Stratakis, E. Tuning cell adhesion by controlling the roughness and wettability of 3D micro/nano silicon structures. Acta Biomaterialia 6, 2711-2720 (2010).

3-39. Albuschies, J. \& Vogel, V. The role of filopodia in the recognition of nanotopographies. Scientific Reports 3, 1658 (2013).

3-40. Wilson, K. et al. Mechanisms of leading edge protrusion in interstitial migration. Nat Commun 4 (2013).

3-41. Ponti, A., Machacek, M., Gupton, S.L., Waterman-Storer, C.M. \& Danuser, G. Two Distinct Actin Networks Drive the Protrusion of Migrating Cells. Science 305, 1782-1786 (2004).

3-42. Yoo, S.K. et al. Differential Regulation of Protrusion and Polarity by PI(3)K during Neutrophil Motility in Live Zebrafish. Developmental Cell 18, 226-236 (2010).

3-43. Prass, M., Jacobson, K., Mogilner, A. \& Radmacher, M. Direct measurement of the lamellipodial protrusive force in a migrating cell. The Journal of Cell Biology 174, 767-772 (2006).

3-44. Small, J.V., Stradal, T., Vignal, E. \& Rottner, K. The lamellipodium: where motility begins. Trends in Cell Biology 12, 112-120 (2002).

3-45. Hoffmann, B. \& Schäfer, C. Filopodial focal complexes direct adhesion and force generation towards filopodia outgrowth. Cell Adhesion \& Migration 4, 190-193 (2010).

3-46. Millard, T.H. \& Martin, P. DYNAMIC ANALYSIS OF FILOPODIAL INTERACTIONS DURING THE ZIPPERING PHASE OF DROSOPHILA DORSAL CLOSURE. Development (Cambridge, England) 135, 621-626 (2008). 
3-47. Partridge, M.A. \& Marcantonio, E.E. Initiation of Attachment and Generation of Mature Focal Adhesions by Integrin-containing Filopodia in Cell Spreading. Molecular Biology of the Cell 17, 4237-4248 (2006).

3-48. Kreis, T.E. \& Birchmeier, W. Stress fiber sarcomeres of fibroblasts are contractile. Cell 22, 555-561 (1980).

4-1. Hata, K.; Yoshida, S.; Fujita, M.; Yasuda, S.; Makimura, T.; Murakami, K.; Shigekawa, H., Self-Assembled Monolayer as a Template to Deposit Silicon Nanoparticles Fabricated by Laser Ablation. The Journal of Physical Chemistry B 2001, 105 (44), 10842-10846.

4-2. Saitow, K.-i., Silicon Nanoclusters Selectively Generated by Laser Ablation in Supercritical Fluid. The Journal of Physical Chemistry B 2005, 109 (9), 3731-3733.

4-3. Jokinen, V.; Aura, S.; Luosujärvi, L.; Sainiemi, L.; Kotiaho, T.; Franssila, S.; Baumann, M., Surface Assisted Laser Desorption/Ionization on Two-Layered Amorphous Silicon Coated Hybrid Nanostructures. J. Am. Soc. Mass Spectrom. 2009, 20 (9), 1723-1730.

4-4. Liu, H.; Huang, Z.; Huang, J.; Xu, S.; Fang, M.; Liu, Y.-g.; Wu, X.; Zhang, S., Morphology controlling method for amorphous silica nanoparticles and jellyfish-like nanowires and their luminescence properties. Scientific Reports 2016, 6, 22459.

4-5. Dalby, M. J.; Riehle, M. O.; Sutherland, D. S.; Agheli, H.; Curtis, A. S. G., Changes in fibroblast morphology in response to nano-columns produced by colloidal lithography. Biomaterials 2004, 25 (23), 5415-5422.

4-6. Low, S. P.; Williams, K. A.; Canham, L. T.; Voelcker, N. H., Evaluation of mammalian cell adhesion on surface-modified porous silicon. Biomaterials 2006, 27 (26), 4538-4546.

4-7 Meng, H.; Zhao, Y.; Dong, J.; Xue, M.; Lin, Y.-S.; Ji, Z.; Mai, W. X.; Zhang, H.; Chang, C. H.; Brinker, C. J.; Zink, J. I.; Nel, A. E., Two-Wave Nanotherapy To Target the Stroma and Optimize Gemcitabine Delivery To a Human Pancreatic Cancer Model in Mice. ACS Nano 2013, 7 (11), 10048-10065.

4-8. Meng, H.; Wang, M.; Liu, H.; Liu, X.; Situ, A.; Wu, B.; Ji, Z.; Chang, C. H.; Nel, A. E., Use of a Lipid-Coated Mesoporous Silica Nanoparticle Platform for Synergistic Gemcitabine and Paclitaxel Delivery to Human Pancreatic Cancer in Mice. ACS Nano 2015, 9 (4), 3540-3557. 
4-9. Xiong, M.-H.; Bao, Y.; Du, X.-J.; Tan, Z.-B.; Jiang, Q.; Wang, H.-X.; Zhu, Y.-H.; Wang, J., Differential Anticancer Drug Delivery with a Nanogel Sensitive to Bacteria-Accumulated Tumor Artificial Environment. ACS Nano 2013, 7 (12), 10636-10645.

4-10. Meng, H.-M.; Zhang, X.; Lv, Y.; Zhao, Z.; Wang, N.-N.; Fu, T.; Fan, H.; Liang, H.; Qiu, L.; Zhu, G.; Tan, W., DNA Dendrimer: An Efficient Nanocarrier of Functional Nucleic Acids for Intracellular Molecular Sensing. ACS Nano 2014, 8 (6), 6171-6181.

4-11. Meng, H.; Xue, M.; Xia, T.; Ji, Z.; Tarn, D. Y.; Zink, J. I.; Nel, A. E., Use of Size and a Copolymer Design Feature To Improve the Biodistribution and the Enhanced Permeability and Retention Effect of Doxorubicin-Loaded Mesoporous Silica Nanoparticles in a Murine Xenograft Tumor Model. ACS Nano 2011, 5 (5), 4131-4144.

4-12. Mendes, P. M., Cellular nanotechnology: making biological interfaces smarter. Chem. Soc. Rev. 2013, 42 (24), 9207-9218.

4-13. Richert, L.; Vetrone, F.; Yi, J.-H.; Zalzal, S. F.; Wuest, J. D.; Rosei, F.; Nanci, A., Surface Nanopatterning to Control Cell Growth. Adv. Mater. 2008, 20 (8), 1488-1492.

4-14. Alimpiev, S.; Grechnikov, A.; Sunner, J.; Karavanskii, V.; Simanovsky, Y.; Zhabin, S.; Nikiforov, S., On the role of defects and surface chemistry for surface-assisted laser desorption ionization from silicon. The Journal of Chemical Physics 2008, 128 (1), 014711.

4-15. Jiang, Y.; Carvalho-de-Souza, J. L.; Wong, R. C. S.; Luo, Z.; Isheim, D.; Zuo, X.; Nicholls, A. W.; Jung, I. W.; Yue, J.; Liu, D.-J.; Wang, Y.; De Andrade, V.; Xiao, X.; Navrazhnykh, L.; Weiss, D. E.; Wu, X.; Seidman, D. N.; Bezanilla, F.; Tian, B., Heterogeneous silicon mesostructures for lipid-supported bioelectric interfaces. Nat Mater 2016, advance online publication.

4-16. Chen, G.; Xie, Y.; Peltier, R.; Lei, H.; Wang, P.; Chen, J.; Hu, Y.; Wang, F.; Yao, X.; Sun, H., Peptide-Decorated Gold Nanoparticles as Functional Nano-Capping Agent of Mesoporous Silica Container for Targeting Drug Delivery. ACS Appl. Mater. Interfaces 2016, 8 (18), 1120411209. 
4-17. Cheng, Y.-J.; Luo, G.-F.; Zhu, J.-Y.; Xu, X.-D.; Zeng, X.; Cheng, D.-B.; Li, Y.-M.; Wu, Y.; Zhang, X.-Z.; Zhuo, R.-X.; He, F., Enzyme-Induced and Tumor-Targeted Drug Delivery System Based on Multifunctional Mesoporous Silica Nanoparticles. ACS Appl. Mater. Interfaces 2015, 7 (17), 9078-9087.

4-18. Zhang, Y.; Hou, Z.; Ge, Y.; Deng, K.; Liu, B.; Li, X.; Li, Q.; Cheng, Z.; Ma, P. a.; Li, C.; Lin, J., DNA-Hybrid-Gated Photothermal Mesoporous Silica Nanoparticles for NIR-Responsive and Aptamer-Targeted Drug Delivery. ACS Appl. Mater. Interfaces 2015, 7 (37), 20696-20706.

4-19. Meng, H.; Liong, M.; Xia, T.; Li, Z.; Ji, Z.; Zink, J. I.; Nel, A. E., Engineered Design of Mesoporous Silica Nanoparticles to Deliver Doxorubicin and P-Glycoprotein siRNA to Overcome Drug Resistance in a Cancer Cell Line. ACS Nano 2010, 4 (8), 4539-4550.

4-20. Rosenholm, J. M.; Meinander, A.; Peuhu, E.; Niemi, R.; Eriksson, J. E.; Sahlgren, C.; Lindén, M., Targeting of Porous Hybrid Silica Nanoparticles to Cancer Cells. ACS Nano 2009, 3 (1), 197-206.

4-21. Zhang, Q.; Liu, F.; Nguyen, K. T.; Ma, X.; Wang, X.; Xing, B.; Zhao, Y., Multifunctional Mesoporous Silica Nanoparticles for Cancer-Targeted and Controlled Drug Delivery. Adv. Funct. Mater. 2012, 22 (24), 5144-5156.

4-22. Ho, D.; Wang, C.-H. K.; Chow, E. K.-H., Nanodiamonds: The intersection of nanotechnology, drug development, and personalized medicine. Science Advances 2015, 1 (7).

4-23. Wang, S.; Wang, H.; Jiao, J.; Chen, K.-J.; Owens, G. E.; Kamei, K.-i.; Sun, J.; Sherman, D. J.; Behrenbruch, C. P.; Wu, H.; Tseng, H.-R., Three-Dimensional Nanostructured Substrates toward Efficient Capture of Circulating Tumor Cells. Angew. Chem., Int. Ed. Engl. 2009, 48 (47), 8970-8973.

4-24. Choi, C.-H.; Hagvall, S. H.; Wu, B. M.; Dunn, J. C. Y.; Beygui, R. E.; “Cj” Kim, C.-J., Cell interaction with three-dimensional sharp-tip nanotopography. Biomaterials 2007, 28 (9), 1672 1679 .

4-25. Carrico, I. S.; Maskarinec, S. A.; Heilshorn, S. C.; Mock, M. L.; Liu, J. C.; Nowatzki, P. J.; Franck, C.; Ravichandran, G.; Tirrell, D. A., Lithographic Patterning of Photoreactive CellAdhesive Proteins. J. Am. Chem. Soc. 2007, 129 (16), 4874-4875. 
4-26. Li, Y.; Huang, G.; Zhang, X.; Wang, L.; Du, Y.; Lu, T. J.; Xu, F., Engineering cell alignment in vitro. Biotechnology Advances 2014, 32 (2), 347-365.

4-27. Li, Y.-C.; Lin, M.-W.; Yen, M.-H.; Fan, S. M.-Y.; Wu, J.-T.; Young, T.-H.; Cheng, J.-Y.; Lin, S.-J., Programmable Laser-Assisted Surface Microfabrication on a Poly(Vinyl Alcohol)Coated Glass Chip with Self-Changing Cell Adhesivity for Heterotypic Cell Patterning. ACS Appl. Mater. Interfaces 2015, 7 (40), 22322-22332.

4-28. Bachhuka, A.; Hayball, J.; Smith, L. E.; Vasilev, K., Effect of Surface Chemical Functionalities on Collagen Deposition by Primary Human Dermal Fibroblasts. ACS Appl. Mater. Interfaces 2015.

4-29. Bettinger, C. J.; Langer, R.; Borenstein, J. T., Engineering Substrate Topography at the Micro- and Nanoscale to Control Cell Function. Angew. Chem., Int. Ed. 2009, 48 (30), 5406-5415. 4-30. Bhuyan, M. K.; Rodriguez-Devora, J. I.; Fraser, K.; Tseng, T.-L. B., Silicon substrate as a novel cell culture device for myoblast cells. Journal of Biomedical Science 2014, 21 (1), 47-47.

4-31. Ranella, A.; Barberoglou, M.; Bakogianni, S.; Fotakis, C.; Stratakis, E., Tuning cell adhesion by controlling the roughness and wettability of 3D micro/nano silicon structures. Acta Biomater. 2010, 6 (7), 2711-2720.

4-32. Santos, P. A.; Rocha, C. S.; Baptista, M. S., Adhesion and proliferation of HeLa and fibroblast cells on chemically-modified gold surfaces. Colloids Surf., B 2014, 123, 429-438.

4-33. Hong, Y.; Fan, H.; Zhang, X., Synthesis and Protein Adsorption of Hierarchical Nanoporous Ultrathin Fibers. The Journal of Physical Chemistry B 2009, 113 (17), 5837-5842.

4-34. Lamour, G.; Eftekhari-Bafrooei, A.; Borguet, E.; Souès, S.; Hamraoui, A., Neuronal adhesion and differentiation driven by nanoscale surface free-energy gradients. Biomaterials 2010, 31 (14), 3762-3771.

4-35. Lamour, G.; Journiac, N.; Souès, S.; Bonneau, S.; Nassoy, P.; Hamraoui, A., Influence of surface energy distribution on neuritogenesis. Colloids Surf., B 2009, 72 (2), 208-218. 
4-36. Albuschies, J.; Vogel, V., The role of filopodia in the recognition of nanotopographies. Scientific Reports 2013, 3, 1658.

4-37. Iversen, T.-G.; Skotland, T.; Sandvig, K., Endocytosis and intracellular transport of nanoparticles: Present knowledge and need for future studies. Nano Today 2011, 6 (2), 176-185.

4-38. Gao, H.; Yang, Z.; Zhang, S.; Cao, S.; Shen, S.; Pang, Z.; Jiang, X., Ligand modified nanoparticles increases cell uptake, alters endocytosis and elevates glioma distribution and internalization. Scientific Reports 2013, 3, 2534.

4-39. Zhang, S.; Gao, H.; Bao, G., Physical Principles of Nanoparticle Cellular Endocytosis. ACS Nano 2015, 9 (9), 8655-8671.

4-40. Napierska, D.; Thomassen, L. C. J.; Lison, D.; Martens, J. A.; Hoet, P. H., The nanosilica hazard: another variable entity. Part. Fibre Toxicol. 2010, 7, 39-39.

4-41. Wu, Y.; Tang, W.; Wang, P.; Liu, C.; Yuan, Y.; Qian, J., Cytotoxicity and Cellular Uptake of Amorphous Silica Nanoparticles in Human Cancer Cells. Part. Part. Syst. Charact. 2015, 32 (7), 779-787.

4-42. Abderrafi, K.; García Calzada, R.; Gongalsky, M. B.; Suárez, I.; Abarques, R.; Chirvony, V. S.; Timoshenko, V. Y.; Ibáñez, R.; Martínez-Pastor, J. P., Silicon Nanocrystals Produced by Nanosecond Laser Ablation in an Organic Liquid. The Journal of Physical Chemistry C 2011, 115 (12), 5147-5151.

4-43. Schaaff, T. G., Laser Desorption and Matrix-Assisted Laser Desorption/Ionization Mass Spectrometry of 29-kDa Au:SR Cluster Compounds. Anal. Chem. 2004, 76 (21), 6187-6196.

4-44. Bhattacharya, S. H.; Raiford, T. J.; Murray, K. K., Infrared Laser Desorption/Ionization on Silicon. Anal. Chem. 2002, 74 (9), 2228-2231.

4-45. Okuno, S.; Arakawa, R.; Okamoto, K.; Matsui, Y.; Seki, S.; Kozawa, T.; Tagawa, S.; Wada, Y., Requirements for Laser-Induced Desorption/Ionization on Submicrometer Structures. Anal. Chem. 2005, 77 (16), 5364-5369. 
4-46. Luo, G.; Chen, Y.; Daniels, H.; Dubrow, R.; Vertes, A., Internal Energy Transfer in Laser Desorption/Ionization from Silicon Nanowires. The Journal of Physical Chemistry B 2006, 110 (27), 13381-13386.

4-47. Sumiya, E.; Shimogawa, H.; Sasaki, H.; Tsutsumi, M.; Yoshita, K. i.; Ojika, M.; Suenaga, K.; Uesugi, M., Cell-Morphology Profiling of a Natural Product Library Identifies Bisebromoamide and Miuraenamide A as Actin Filament Stabilizers. ACS Chem. Biol. 2011, 6 (5), 425-431.

4-48. Milroy, L.-G.; Rizzo, S.; Calderon, A.; Ellinger, B.; Erdmann, S.; Mondry, J.; Verveer, P.; Bastiaens, P.; Waldmann, H.; Dehmelt, L.; Arndt, H.-D., Selective Chemical Imaging of Static Actin in Live Cells. J. Am. Chem. Soc. 2012, 134 (20), 8480-8486.

4-49. Tojkander, S.; Gateva, G.; Lappalainen, P., Actin stress fibers - assembly, dynamics and biological roles. J. Cell Sci. 2012, 125 (8), 1855-1864.

4-50. Hakkinen, K. M.; Harunaga, J. S.; Doyle, A. D.; Yamada, K. M., Direct Comparisons of the Morphology, Migration, Cell Adhesions, and Actin Cytoskeleton of Fibroblasts in Four Different Three-Dimensional Extracellular Matrices. Tissue Engineering. Part A 2011, 17 (5-6), 713-724.

4-51. Lopacinska, J. M.; Gradinaru, C.; Wierzbicki, R.; Kobler, C.; Schmidt, M. S.; Madsen, M. T.; Skolimowski, M.; Dufva, M.; Flyvbjerg, H.; Molhave, K., Cell motility, morphology, viability and proliferation in response to nanotopography on silicon black. Nanoscale 2012, 4 (12), 37393745 .

4-52. Zhang, F.; Jiang, Y.; Liu, X.; Meng, J.; Zhang, P.; Liu, H.; Yang, G.; Li, G.; Jiang, L.; Wan, L.-J.; Hu, J.-S.; Wang, S., Hierarchical Nanowire Arrays as Three-Dimensional Fractal Nanobiointerfaces for High Efficient Capture of Cancer Cells. Nano Letters 2016, 16 (1), 766-772. 4-53. Chen, W.; Weng, S.; Zhang, F.; Allen, S.; Li, X.; Bao, L.; Lam, R. H. W.; Macoska, J. A.; Merajver, S. D.; Fu, J., Nanoroughened Surfaces for Efficient Capture of Circulating Tumor Cells without Using Capture Antibodies. ACS Nano 2013, 7 (1), 566-575.

4-54. Lord, M. S.; Foss, M.; Besenbacher, F., Influence of nanoscale surface topography on protein adsorption and cellular response. Nano Today 2010, 5 (1), 66-78. 
4-55. Zhao, L.; Mei, S.; Chu, P. K.; Zhang, Y.; Wu, Z., The influence of hierarchical hybrid micro/nano-textured titanium surface with titania nanotubes on osteoblast functions. Biomaterials 2010, 31 (19), 5072-5082.

4-56. Clemments, A. M.; Botella, P.; Landry, C. C., Protein Adsorption From Biofluids on Silica Nanoparticles: Corona Analysis as a Function of Particle Diameter and Porosity. ACS Appl. Mater. Interfaces 2015, 7 (39), 21682-21689.

4-57. Rechendorff, K.; Hovgaard, M. B.; Foss, M.; Zhdanov, V. P.; Besenbacher, F., Enhancement of Protein Adsorption Induced by Surface Roughness. Langmuir 2006, 22 (26), 10885-10888.

4-58. Arima, Y.; Iwata, H., Effect of wettability and surface functional groups on protein adsorption and cell adhesion using well-defined mixed self-assembled monolayers. Biomaterials 2007, 28 (20), 3074-3082.

4-59. Sigal, G. B.; Mrksich, M.; Whitesides, G. M., Effect of Surface Wettability on the Adsorption of Proteins and Detergents. J. Am. Chem. Soc. 1998, 120 (14), 3464-3473.

4-60. Ngandu Mpoyi, E.; Cantini, M.; Reynolds, P. M.; Gadegaard, N.; Dalby, M. J.; SalmerónSánchez, M., Protein Adsorption as a Key Mediator in the Nanotopographical Control of Cell Behavior. ACS Nano 2016.

4-61. Yang, J.; McNamara, L. E.; Gadegaard, N.; Alakpa, E. V.; Burgess, K. V.; Meek, R. M. D.; Dalby, M. J., Nanotopographical Induction of Osteogenesis through Adhesion, Bone Morphogenic Protein Cosignaling, and Regulation of MicroRNAs. ACS Nano 2014, 8 (10), 9941-9953.

4-62. Dalal, C.; Saha, A.; Jana, N. R., Nanoparticle Multivalency Directed Shifting of Cellular Uptake Mechanism. The Journal of Physical Chemistry C 2016, 120 (12), 6778-6786.

4-63. Slowing, I.; Trewyn, B. G.; Lin, V. S. Y., Effect of Surface Functionalization of MCM-41Type Mesoporous Silica Nanoparticles on the Endocytosis by Human Cancer Cells. J. Am. Chem. Soc. 2006, 128 (46), 14792-14793.

4-64. Villanueva, A.; de la Presa, P.; Alonso, J. M.; Rueda, T.; Martínez, A.; Crespo, P.; Morales, M. P.; Gonzalez-Fernandez, M. A.; Valdés, J.; Rivero, G., Hyperthermia HeLa Cell Treatment with Silica-Coated Manganese Oxide Nanoparticles. The Journal of Physical Chemistry C 2010, 114 (5), 1976-1981. 
4-65. Panagiotakopoulou, M.; Bergert, M.; Taubenberger, A.; Guck, J.; Poulikakos, D.; Ferrari, A., A Nanoprinted Model of Interstitial Cancer Migration Reveals a Link between Cell Deformability and Proliferation. ACS Nano 2016.

4-66. Charras, G.; Sahai, E., Physical influences of the extracellular environment on cell migration. Nat Rev Mol Cell Biol 2014, 15 (12), 813-824.

4-67. Lesniak, A.; Salvati, A.; Santos-Martinez, M. J.; Radomski, M. W.; Dawson, K. A.; Åberg, C., Nanoparticle Adhesion to the Cell Membrane and Its Effect on Nanoparticle Uptake Efficiency. J. Am. Chem. Soc. 2013, 135 (4), 1438-1444.

4-68. Walkey, C. D.; Olsen, J. B.; Guo, H.; Emili, A.; Chan, W. C. W., Nanoparticle Size and Surface Chemistry Determine Serum Protein Adsorption and Macrophage Uptake. J. Am. Chem. Soc. 2012, 134 (4), 2139-2147.

4-69. Zhang, H.; Dunphy, D. R.; Jiang, X.; Meng, H.; Sun, B.; Tarn, D.; Xue, M.; Wang, X.; Lin, S.; Ji, Z.; Li, R.; Garcia, F. L.; Yang, J.; Kirk, M. L.; Xia, T.; Zink, J. I.; Nel, A.; Brinker, C. J., Processing Pathway Dependence of Amorphous Silica Nanoparticle Toxicity: Colloidal vs Pyrolytic. J. Am. Chem. Soc. 2012, 134 (38), 15790-15804.

4-70. Webster, M.; Witkin, K. L.; Cohen-Fix, O., Sizing up the nucleus: nuclear shape, size and nuclear-envelope assembly. J. Cell Sci. 2009, 122 (10), 1477-1486.

4-71. Liu, H. C.; Chen, G. G.; Vlantis, A. C.; Tse, G. M.; Chan, A. T. C.; van Hasselt, C. A., Inhibition of apoptosis in human laryngeal cancer cells by E6 and E7 oncoproteins of human papillomavirus 16. J. Cell. Biochem. 2008, 103 (4), 1125-1143.

4-72. Liu, Y.; McKalip, A.; Herman, B., Human papillomavirus type 16 E6 and HPV-16 E6/E7 sensitize human keratinocytes to apoptosis induced by chemotherapeutic agents: Roles of p53 and caspase activation. J. Cell. Biochem. 2000, 78 (2), 334-349.

5-1. Nishimura, H.; Ritchie, K.; Kasai, R. S.; Goto, M.; Morone, N.; Sugimura, H.; Tanaka, K.; Sase, I.; Yoshimura, A.; Nakano, Y.; Fujiwara, T. K.; Kusumi, A., Biocompatible fluorescent silicon nanocrystals for single-molecule tracking and fluorescence imaging. The Journal of Cell Biology 2013, 202 (6), 967-983. 
5-2. Alexis J. Torres, M. W., David Holowka, and Barbara Baird, Nanobiotechnology and Cell Biology: Micro- and Nanofabricated Surfaces to Investigate Receptor-Mediated Signaling. Annual Review of Biophysics 2008, 37 (1), 265-288.

5-3. Fernandez-Suarez, M.; Ting, A. Y., Fluorescent probes for super-resolution imaging in living cells. Nat Rev Mol Cell Biol 2008, 9 (12), 929-943.

5-4. Dickson, R. M.; Cubitt, A. B.; Tsien, R. Y.; Moerner, W. E., On/off blinking and switching behaviour of single molecules of green fluorescent protein. Nature 1997, 388 (6640), 355-358.

5-5. Buschmann, V.; Weston, K. D.; Sauer, M., Spectroscopic Study and Evaluation of RedAbsorbing Fluorescent Dyes. Bioconjugate Chemistry 2003, 14 (1), 195-204.

5-6. Howarth, M.; Liu, W.; Puthenveetil, S.; Zheng, Y.; Marshall, L. F.; Schmidt, M. M.; Wittrup, K. D.; Bawendi, M. G.; Ting, A. Y., Monovalent, reduced-size quantum dots for imaging receptors on living cells. Nat Meth 2008, 5 (5), 397-399.

5-7. Claridge, S. A.; Schwartz, J. J.; Weiss, P. S., Electrons, Photons, and Force: Quantitative Single-Molecule Measurements from Physics to Biology. ACS Nano 2011, 5 (2), 693-729.

5-8. Wang, X.; Ren, X.; Kahen, K.; Hahn, M. A.; Rajeswaran, M.; Maccagnano-Zacher, S.; Silcox, J.; Cragg, G. E.; Efros, A. L.; Krauss, T. D., Non-blinking semiconductor nanocrystals. Nature 2009, 459 (7247), 686-689.

5-9. Erogbogbo, F.; Yong, K.-T.; Hu, R.; Law, W.-C.; Ding, H.; Chang, C.-W.; Prasad, P. N.; Swihart, M. T., Biocompatible Magnetofluorescent Probes: Luminescent Silicon Quantum Dots Coupled with Superparamagnetic Iron(III) Oxide. ACS Nano 2010, 4 (9), 5131-5138.

5-10. Erogbogbo, F.; Yong, K.-T.; Roy, I.; Hu, R.; Law, W.-C.; Zhao, W.; Ding, H.; Wu, F.; Kumar, R.; Swihart, M. T.; Prasad, P. N., In Vivo Targeted Cancer Imaging, Sentinel Lymph Node Mapping and Multi-Channel Imaging with Biocompatible Silicon Nanocrystals. ACS Nano 2011, $5(1), 413-423$.

5-11. Erogbogbo, F.; Yong, K.-T.; Roy, I.; Xu, G.; Prasad, P. N.; Swihart, M. T., Biocompatible Luminescent Silicon Quantum Dots for Imaging of Cancer Cells. ACS Nano 2008, 2 (5), 873-878. 
5-12. Hutter, E.; Boridy, S.; Labrecque, S.; Lalancette-Hébert, M.; Kriz, J.; Winnik, F. M.; Maysinger, D., Microglial Response to Gold Nanoparticles. ACS Nano 2010, 4 (5), 2595-2606.

5-13. Cheng, J.; Fernando, K. A. S.; Veca, L. M.; Sun, Y.-P.; Lamond, A. I.; Lam, Y. W.; Cheng, S. H., Reversible Accumulation of PEGylated Single-Walled Carbon Nanotubes in the Mammalian Nucleus. ACS Nano 2008, 2 (10), 2085-2094.

5-14. Cheng, X.; Lowe, S. B.; Ciampi, S.; Magenau, A.; Gaus, K.; Reece, P. J.; Gooding, J. J., Versatile "Click Chemistry" Approach to Functionalizing Silicon Quantum Dots: Applications toward Fluorescent Cellular Imaging. Langmuir 2014, 30 (18), 5209-5216.

5-15. Cheng, X.; Lowe, S. B.; Reece, P. J.; Gooding, J. J., Colloidal silicon quantum dots: from preparation to the modification of self-assembled monolayers (SAMs) for bio-applications. Chemical Society Reviews 2014, 43 (8), 2680-2700.

5-16. Kim, B.; Han, G.; Toley, B. J.; Kim, C.-k.; Rotello, V. M.; Forbes, N. S., Tuning payload delivery in tumour cylindroids using gold nanoparticles. Nat Nano 2010, 5 (6), 465-472.

5-17. Hardman, R., A Toxicologic Review of Quantum Dots: Toxicity Depends on Physicochemical and Environmental Factors. Environmental Health Perspectives 2006, 114 (2), 165-172.

5-18. Hoshino, A.; Hanada, S.; Yamamoto, K., Toxicity of nanocrystal quantum dots: the relevance of surface modifications. Archives of Toxicology 2011, 85 (7), 707.

5-19. Winnik, F. M.; Maysinger, D., Quantum Dot Cytotoxicity and Ways To Reduce It. Accounts of Chemical Research 2013, 46 (3), 672-680.

5-20. Derfus, A. M.; Chan, W. C. W.; Bhatia, S. N., Probing the Cytotoxicity of Semiconductor Quantum Dots. Nano Letters 2004, 4 (1), 11-18.

5-21. Gao, H.; Dong, H.; Cao, X.; Fu, X.; Zhu, Y.; Mao, C.; Wang, Y., Effective Spatial Separation of PC12 and NIH3T3 Cells by the Microgrooved Surface of Biocompatible Polymer Substrates. Langmuir 2015, 31 (24), 6797-6806. 
5-22. Gao, H.; Yang, Z.; Zhang, S.; Cao, S.; Shen, S.; Pang, Z.; Jiang, X., Ligand modified nanoparticles increases cell uptake, alters endocytosis and elevates glioma distribution and internalization. Scientific Reports 2013, 3, 2534.

5-23. Gao, X.; Cui, Y.; Levenson, R. M.; Chung, L. W. K.; Nie, S., In vivo cancer targeting and imaging with semiconductor quantum dots. Nat Biotech 2004, 22 (8), 969-976.

5-24. Tilley, R. D.; Warner, J. H.; Yamamoto, K.; Matsui, I.; Fujimori, H., Micro-emulsion synthesis of monodisperse surface stabilized silicon nanocrystals. Chemical Communications 2005, (14), 1833-1835.

5-25. Choi, J.; Wang, N. S.; Reipa, V., Conjugation of the Photoluminescent Silicon Nanoparticles to Streptavidin. Bioconjugate Chemistry 2008, 19 (3), 680-685.

5-26. Fu, A.; Gu, W.; Larabell, C.; Alivisatos, A. P., Semiconductor nanocrystals for biological imaging. Current Opinion in Neurobiology 2005, 15 (5), 568-575.

5-27. Alivisatos, A. P., Semiconductor Clusters, Nanocrystals, and Quantum Dots. Science 1996, 271 (5251), 933-937.

5-28. Michalet, X.; Pinaud, F. F.; Bentolila, L. A.; Tsay, J. M.; Doose, S.; Li, J. J.; Sundaresan, G.; Wu, A. M.; Gambhir, S. S.; Weiss, S., Quantum Dots for Live Cells, in Vivo Imaging, and Diagnostics. Science 2005, 307 (5709), 538-544.

5-29. Dubertret, B.; Skourides, P.; Norris, D. J.; Noireaux, V.; Brivanlou, A. H.; Libchaber, A., In Vivo Imaging of Quantum Dots Encapsulated in Phospholipid Micelles. Science 2002, 298 (5599), 1759-1762.

5-30. Biju, V.; Mundayoor, S.; Omkumar, R. V.; Anas, A.; Ishikawa, M., Bioconjugated quantum dots for cancer research: Present status, prospects and remaining issues. Biotechnology Advances 2010, 28 (2), 199-213.

5-31. Ishikawa, M.; Biju, V., Chapter 2 - Luminescent Quantum Dots, Making Invisibles Visible in Bioimaging. In Progress in Molecular Biology and Translational Science, Antonio, V., Ed. Academic Press: 2011; Vol. Volume 104, pp 53-99. 
5-32. Kim, J. A.; Aberg, C.; Salvati, A.; Dawson, K. A., Role of cell cycle on the cellular uptake and dilution of nanoparticles in a cell population. Nat Nano 2012, 7 (1), 62-68.

5-33. Maus, L.; Dick, O.; Bading, H.; Spatz, J. P.; Fiammengo, R., Conjugation of Peptides to the Passivation Shell of Gold Nanoparticles for Targeting of Cell-Surface Receptors. ACS Nano 2010, 4 (11), 6617-6628.

5-34. Verma, A.; Uzun, O.; Hu, Y.; Hu, Y.; Han, H.-S.; Watson, N.; Chen, S.; Irvine, D. J.; Stellacci, F., Surface-structure-regulated cell-membrane penetration by monolayer-protected nanoparticles. Nat Mater 2008, 7 (7), 588-595.

5-35. So, M.-K.; Xu, C.; Loening, A. M.; Gambhir, S. S.; Rao, J., Self-illuminating quantum dot conjugates for in vivo imaging. Nat Biotech 2006, 24 (3), 339-343.

5-36. Huang, K.; Ma, H.; Liu, J.; Huo, S.; Kumar, A.; Wei, T.; Zhang, X.; Jin, S.; Gan, Y.; Wang, P. C.; He, S.; Zhang, X.; Liang, X.-J., Size-Dependent Localization and Penetration of Ultrasmall Gold Nanoparticles in Cancer Cells, Multicellular Spheroids, and Tumors in Vivo. ACS Nano 2012, 6 (5), 4483-4493.

5-37. Paine, P. L.; Moore, L. C.; Horowitz, S. B., Nuclear envelope permeability. Nature 1975, 254 (5496), 109-114.

5-38. He, C.; Hu, Y.; Yin, L.; Tang, C.; Yin, C., Effects of particle size and surface charge on cellular uptake and biodistribution of polymeric nanoparticles. Biomaterials 2010, 31 (13), 36573666.

5-39. Zhang, S.; Li, J.; Lykotrafitis, G.; Bao, G.; Suresh, S., Size-Dependent Endocytosis of Nanoparticles. Advanced Materials 2009, 21 (4), 419-424.

5-40. Chinnathambi, S.; Chen, S.; Ganesan, S.; Hanagata, N., Silicon Quantum Dots for Biological Applications. Advanced Healthcare Materials 2014, 3 (1), 10-29.

5-41. Zhang, Y.; Wang, T.-H., Quantum Dot Enabled Molecular Sensing and Diagnostics. Theranostics 2012, 2 (7), 631-654. 
5-42. Shiohara, A.; Prabakar, S.; Faramus, A.; Hsu, C.-Y.; Lai, P.-S.; Northcote, P. T.; Tilley, R. D., Sized controlled synthesis, purification, and cell studies with silicon quantum dots. Nanoscale 2011, 3 (8), 3364-3370.

5-43. Ye, H.-L.; Cai, S.-J.; Li, S.; He, X.-W.; Li, W.-Y.; Li, Y.-H.; Zhang, Y.-K., One-Pot Microwave Synthesis of Water-Dispersible, High Fluorescence Silicon Nanoparticles and Their Imaging Applications in Vitro and in Vivo. Analytical Chemistry 2016, 88 (23), 11631-11638.

5-44. Deng, D.; Qu, L.; Li, Y.; Gu, Y., Versatile Self-Assembly of Water-Soluble Thiol-Capped CdTe Quantum Dots: External Destabilization and Internal Stability of Colloidal QDs. Langmuir 2013, 29 (34), 10907-10914.

5-45. Sun, D.; Sue, H.-J.; Miyatake, N., Optical Properties of ZnO Quantum Dots in Epoxy with Controlled Dispersion. The Journal of Physical Chemistry C 2008, 112 (41), 16002-16010.

5-46. Komoto, A.; Maenosono, S.; Yamaguchi, Y., Oscillating Fluorescence in an Unstable Colloidal Dispersion of CdSe/ZnS Core/Shell Quantum Dots. Langmuir 2004, 20 (20), 8916-8923.

6-1. Q. Ma and K. Nakazato, Biosensors \& bioelectronics, 2014, 51, 362-365.

6-2. R. Chauhan, J. Singh, P. R. Solanki, T. Manaka, M. Iwamoto, T. Basu and B. D. Malhotra, Sensors and Actuators B: Chemical, 2016, 222, 804-814.

6-3. R. Uddin, R. Burger, M. Donolato, J. Fock, M. Creagh, M. F. Hansen and A. Boisen, Biosensors \& bioelectronics, 2016, 85, 351-357.

6-4. F. Chen, B. R. Flaherty, C. E. Cohen, D. S. Peterson and Y. Zhao, Nanomedicine : nanotechnology, biology, and medicine, 2016, 12, 1445-1451.

6-5. Y. Wang and J. Irudayaraj, Philosophical transactions of the Royal Society of London. Series B, Biological sciences, 2013, 368, 20120026.

6-6. R. Xiao, C. W. Wang, A. N. Zhu and F. Long, Biosensors \& bioelectronics, 2016, 79, 661668.

6-7. S. Z. Oo, G. Silva, F. Carpignano, A. Noual, K. Pechstedt, L. Mateos, J. A. Grant-Jacob, B. Brocklesby, P. Horak, M. Charlton, S. A. Boden and T. Melvin, Sensing and Bio-Sensing Research, 2016, 7, 133-140. 
6-8. G. Q. Wallace, M. S. Zuin, M. Tabatabaei, P. Gobbo, F. Lagugne-Labarthet and M. S. Workentin, The Analyst, 2015, 140, 7278-7282.

6-9. X. Zheng, Q. Fu, H. Guo, M. Wu and Y. Lei, Nanomedicine : nanotechnology, biology, and medicine, 2016, 12.

6-10. H. N. Wang, A. M. Fales and T. Vo-Dinh, Nanomedicine : nanotechnology, biology, and medicine, 2015, 11, 811-814.

6-11. A. R. Bizzarri and S. Cannistraro, Nanomedicine : nanotechnology, biology, and medicine, 2007, 3, 306-310.

6-12. Q. Yao, Z. Zhang, F. Cao, C. Feng, Y. Zhao and X. Wang, Nanomedicine : nanotechnology, biology, and medicine, 2016, 12, 1.

6-13. A. Convertino, V. Mussi and L. Maiolo, Scientific reports, 2016, 6, 25099.

6-14. T. Li, K. Wu, T. Rindzevicius, Z. Wang, L. Schulte, M. S. Schmidt, A. Boisen and S. Ndoni, ACS applied materials \& interfaces, 2016, 8, 15668-15675.

6-15. C. Zhang, B. Y. Man, S. Z. Jiang, C. Yang, M. Liu, C. S. Chen, S. C. Xu, H. W. Qiu and Z. Li, Applied Surface Science, 2015, 347, 668-672.

6-16. C. Zhang, S. Z. Jiang, C. Yang, C. H. Li, Y. Y. Huo, X. Y. Liu, A. H. Liu, Q. Wei, S. S. Gao, X. G. Gao and B. Y. Man, Scientific reports, 2016, 6, 25243.

6-17. L. Yang, M. Gong, X. Jiang, Y. Chen, X. Han, K. Song, X. Sun, Y. Zhang and B. Zhao, Colloids and Surfaces A: Physicochemical and Engineering Aspects, 2016, 508, 142-149.

6-18. L. Chang, D. Xu and X. Xue, Journal of Materials Science: Materials in Electronics, 2015, 27, 1014-1019.

6-19. J. R. Lombardi and R. L. Birke, The Journal of Physical Chemistry C, 2014, 118, 1112011130.

6-20. L. Yang, M. Gong, X. Jiang, D. Yin, X. Qin, B. Zhao and W. Ruan, Journal of Raman Spectroscopy, 2015, 46, 287-292. 
6-21. X. Zeng, S. Yan, J. Cui, H. Liu, J. Dong, W. Xia, M. Zhou and H. Chen, Journal of nanoparticle research : an interdisciplinary forum for nanoscale science and technology, 2015, 17, 188.

6-22. H. Mao, Y. Chen and J. Wang, Optical and Quantum Electronics, 2015, 47, 2811-2819.

6-23. R. Zhang, P.-G. Yin, N. Wang and L. Guo, Solid State Sciences, 2009, 11, 865-869.

6-24. C. Novara, S. Dalla Marta, A. Virga, A. Lamberti, A. Angelini, A. Chiadò, P. Rivolo, F. Geobaldo, V. Sergo, A. Bonifacio and F. Giorgis, The Journal of Physical Chemistry C, 2016, 120, 16946-16953.

6-25. J. A. Powell, K. Venkatakrishnan and B. Tan, Scientific reports, 2016, 6, 19663.

6-26. J. A. Powell, K. Venkatakrishnan and B. Tan, J Mater Chem B, 2016, 4, 5713-5728.

6-27. H. Zhang, C. Wang, K. Wang, X. Xuan, Q. Lv and K. Jiang, Biosensors \& bioelectronics, 2016, 85, 96-102.

6-28. K. Xu and P. J. Thornalley, Biochemical Pharmacology, 2001, 61, 12.

6-29. S. Hong and X. Li, Journal of Nanomaterials, 2013, 2013, 1-9.

6-30. J. Wang, X. Wu, C. Wang, Z. Rong, H. Ding, H. Li, S. Li, N. Shao, P. Dong, R. Xiao and S. Wang, ACS applied materials \& interfaces, 2016, 8, 19958-19967.

6-31. J. Q. Xu, H. H. Duo, Y. G. Zhang, X. W. Zhang, W. Fang, Y. L. Liu, A. G. Shen, J. M. Hu and W. H. Huang, Analytical chemistry, 2016, 88, 3789-3795.

6-32. X. Hong, X. Chu, P. Zou, Y. Liu and G. Yang, Biosensors \& bioelectronics, 2010, 26, $918-$ 922.

6-33. A. Campion and P. Kambhampati, Chemical Society Reviews, 1998, 27, 9.

6-34. A. Campion, J. E. Ivanecky, C. M. Child and M. Foster, Journal of the American Chemical Society, 1995, 117, 11807-11808.

6-35. J. R. Lombardi and R. L. Birke, J Chem Phys, 2007, 126, 244709. 
6-36. P. L. Stiles, J. A. Dieringer, N. C. Shah and R. P. Van Duyne, Annu Rev Anal Chem (Palo Alto Calif), 2008, 1, 601-626.

6-37. J. Jiang, K. Bosnick, M. Maillard and L. Brus, J Phys Chem B, 2003, 107, 9964-9972.

6-38. K. Tanabe, J Phys Chem C, 2008, 112, 15721-15728.

6-39. H. Raether, Surface Plasmons on Smooth and Rough Surfaces and on Gratings, Springer Berlin Heidelberg, 1988.

6-40. S. A. Maier and H. A. Atwater, Journal of Applied Physics, 2005, 98, 011101.

6-41. J. S. Bains and C. A. Shaw, Brain Research Reviews, 1997, 25, 23.

6-42. S. Chinnakkannu Vijayakumar, K. Venkatakrishnan and B. Tan, ACS applied materials \& interfaces, 2017, DOI: 10.1021/acsami.6b13576.

6-43. G. G. Huang, X. X. Han, M. K. Hossain and Y. Ozaki, Analytical chemistry, 2009, 81, 58815888.

6-44. J. De Gelder, K. De Gussem, P. Vandenabeele and L. Moens, Journal of Raman Spectroscopy, 2007, 38, 1133-1147.

6-45. W. Qian and S. Krimm, Biopolymers, 1994, 34, 1377-1394.

6-46. A. Brambilla, A. Philippidis, A. Nevin, D. Comelli, G. Valentini and D. Anglos, Journal of Molecular Structure, 2013, 1044, 121-127.

6-47. S. Cong, Y. Yuan, Z. Chen, J. Hou, M. Yang, Y. Su, Y. Zhang, L. Li, Q. Li, F. Geng and Z. Zhao, Nature communications, 2015, 6, 7800.

6-48. G. Das, E. Battista, G. Manzo, F. Causa, P. A. Netti and E. Di Fabrizio, ACS applied materials \& interfaces, 2015, 7, 23597-23604.

6-49. L. Zhou, J. Zhou, Z. Feng, F. Wang, S. Xie and S. Bu, The Analyst, 2016, 141, 2534-2541.

6-50. H. H. An, W. B. Han, Y. Kim, H.-S. Kim, Y. Oh and C. S. Yoon, Journal of Raman Spectroscopy, 2014, 45, 292-298. 
6-51. D. Lin, Z. Wu, S. Li, W. Zhao, C. Ma, J. Wang, Z. Jiang, Z. Zhong, Y. Zheng and X. Yang, ACS Nano, 2017, DOI: 10.1021/acsnano.6b06778.

6-52. P. Thakur, B. Tan and K. Venkatakrishnan, Solar Energy Materials and Solar Cells, 2017, 164, 165-174.

6-53. L. Qin, X. Li, S. Z. Kang and J. Mu, Colloids and surfaces. B, Biointerfaces, 2015, 126, 210216.

6-54. D.-Y. Hong, S. K. Kim and Y.-U. Kwon, The Journal of Physical Chemistry C, 2015, 119, 22611-22617. 